THE UNIVERSITY OF CHICAGO

\title{
A DEMONSTRATION OF LIGHT-AUGMENTED CALORIMETRY FOR LOW-ENERGY ELECTRONS IN LIQUID ARGON
}

\author{
A DISSERTATION SUBMITTED TO \\ THE FACULTY OF THE DIVISION OF THE PHYSICAL SCIENCES \\ IN CANDIDACY FOR THE DEGREE OF \\ DOCTOR OF PHILOSOPHY
}

DEPARTMENT OF PHYSICS

BY

WILLIAM M. FOREMAN

CHICAGO, ILLINOIS

MARCH 2019 
Copyright (c) 2019 by William M. Foreman

All Rights Reserved 
For my family. 


\section{Table of Contents}

List of Figures ......................... . vii

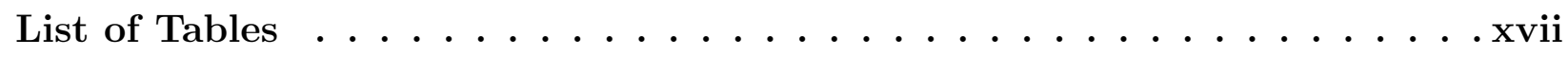

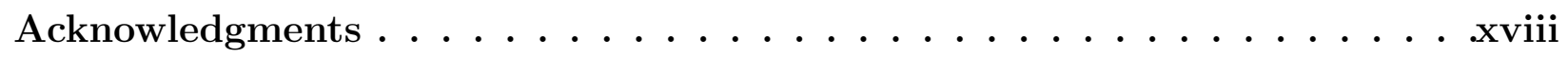

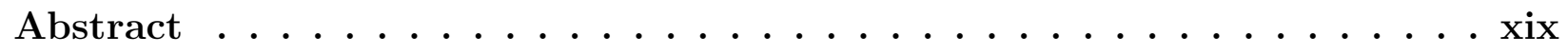

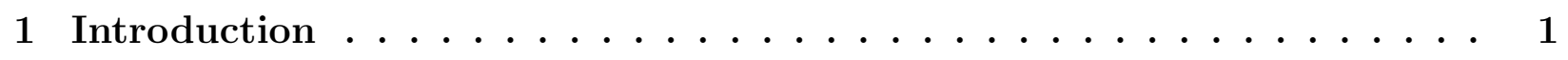

2 Detecting Neutrinos with Liquid Argon TPCs . . . . . . . . . . . . . 5

2.1 Neutrino physics: what we know . . . . . . . . . . . . . . . . 5

2.2 Open questions in neutrino physics . . . . . . . . . . . . . . . . 11

2.3 LArTPCs for neutrino detection . . . . . . . . . . . . . . . . . . . . . . . 13

$2.4 \quad$ Studies enabled by a large underground LArTPC . . . . . . . . . . . . . . 15

2.5 Motivation for low-energy calorimetric capabilities . . . . . . . . . . . . . . . 19

2.6 The case for scintillation light . . . . . . . . . . . . . . . . . . . . 22

3 Charge and Light Production in Liquid Argon . . . . . . . . . . . . . . . . 24

$3.1 \quad$ Energy loss by charged particles in matter . . . . . . . . . . . . . . . . . . . 24

3.2 Ionization, scintillation, and recombination $\ldots \ldots \ldots$. . . . . . . 27

3.3 Quenching effects from impurities in LArTPCs . . . . . . . . . . . . . . . . 34

3.4 Implications for energy reconstruction . . . . . . . . . . . . . . . . . 37

4 The LArIAT Testbeam Experiment $\ldots$. . . . . . . . . . . . . . . 39

$4.1 \quad$ Motivation for LArIAT: physics and R\&D goals . . . . . . . . . . . . . . . . 39

4.2 Beamline . . . . . . . . . . . . . . . . . . . . . . . . 41

4.3 Cryogenic system $\ldots \ldots \ldots$. . . . . . . . . . . . . . . . . . . . 42

4.4 Time projection chamber . . . . . . . . . . . . . . . . . . . . 44 
4.5 Photon detection system . . . . . . . . . . . . . . . . . . . . 45

4.6 Data acquisition . . . . . . . . . . . . . . . . . . . . . . 52

4.7 Light-based Michel electron trigger . . . . . . . . . . . . . . . . . . . . 54

5 Reconstruction of Michel Electrons . . . . . . . . . . . . . . 57

5.1 Overview of muon decay and capture . . . . . . . . . . . . . . . 57

5.2 The Michel electron data samples . . . . . . . . . . . . . . . . . . . . . . . . 59

5.3 Charge reconstruction $\ldots \ldots \ldots \ldots$. . . . . . . . . . . . . 59

5.4 Optical reconstruction . . . . . . . . . . . . . . . . . 71

5.5 Muon decay time spectra and measurement of capture lifetime . . . . . . . . 77

6 Detector Calibrations . . . . . . . . . . . . . . . . . 82

6.1 Charge-based calibrations . . . . . . . . . . . . . . . . . 82

6.2 Light-based calibrations . . . . . . . . . . . . . . . . . . . 88

7 Monte Carlo Simulation . . . . . . . . . . . . . . . . . . 94

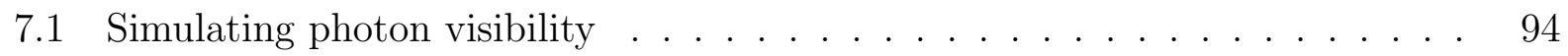

$7.2 \quad$ Particle propagation and energy loss with Geant4 . . . . . . . . . . . . . . . 99

7.3 Charge and scintillation production . . . . . . . . . . . . . . . 100

7.4 Charge drift and signal formation . . . . . . . . . . . . . . . . . . . . 102

7.5 Scintillation signal formation . . . . . . . . . . . . . . . . . . . . 102

7.6 Optical smearing and trigger efficiency . . . . . . . . . . . . . . . . . 104

8 Calorimetry Results for Low-Energy Electrons in LArIAT . . . . . . . . . 110

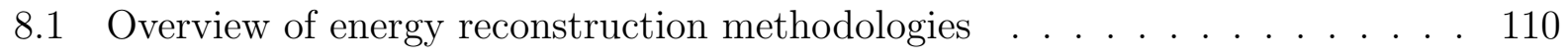

8.2 Event selection . . . . . . . . . . . . . . . . . . . . . 112

8.3 Reconstructing charge and light . . . . . . . . . . . . . . . . . . . 115

$8.4 \quad$ Electron energy spectra using charge and light . . . . . . . . . . . . . . . . . 121

8.5 Maximum likelihood method for $\mathrm{Q}+\mathrm{L}$. . . . . . . . . . . . . . . . . 124 
$8.6 \quad$ Monte Carlo energy resolution . . . . . . . . . . . . . . . . . . . . . . . . . . 131

8.6 .1 Michel electrons . . . . . . . . . . . . . . . . . . . . . . . 132

8.6 .2 Isolated electrons . . . . . . . . . . . . . . . . . . . . . . . . . 133

8.7 Discussion of systematic uncertainties . . . . . . . . . . . . . . . . 135

9 Calorimetric Potential in Future LArTPCs . . . . . . . . . . . . . . 139

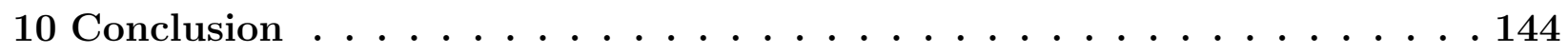

A Determining the Quenched Scintillation Lifetime in Ar . . . . . . . 146

B Electron Energy Resolution: Supporting Plots . . . . . . . . . . . . . 148

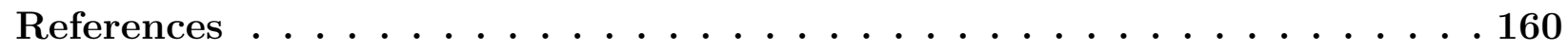




\section{List of Figures}

2.1 The fundamental particles of nature as described by the Standard Model [14].] . 6

2.2 A schematic of the neutrino squared-mass spectrum assuming three mass eigen-

states and a "normal" mass-ordering. The flavor content of each mass state is illustrated based on existing experimental results (excluding those from LSND) [17]. 10

2.3 Illustration of charged particles emerging from a neutrino interaction and producing ionization tracks in a LArTPC. . . . . . . . . . . . . . . . . . . . . . . 14

2.4 The DUNE and LBNF project. A neutrino beam is produced at Fermilab and directed through the earth toward the DUNE far detector located $1.5 \mathrm{~km}$ underground [10]. . . . . . . . . . . . . . . . . . . . . . . . . . . . . . . . . 16

2.5 Core-collapse supernova $\nu$ signals expected based on the Basel model for a 10.8 solar-mass star. The top row shows the intensity over time, while the bottom row shows the average energy over time, separated by neutrino flavor [25, 26]. . . . . 17

2.6 Cross-sections for neutrino interactions relevant to supernova emission [29, 30]. . 18

2.7 Simulated time-dependent $\nu$ signal in $40 \mathrm{kt}$ of LAr from an electron capture SN $10 \mathrm{kpc}$ away (left), and the energy spectrum of $\nu$ integrated over arrival times (right) [31]. . . . . . . . . . . . . . . . . . . . . . . . . . . . . . . 19

2.8 Visible energy expected from solar ${ }^{8} \mathrm{~B}$ and hep $\nu$ signal and background in DUNE over a total exposure of 100 kton years for CC-interactions and elastic scattering (ES) interactions. Looking either inside (left) or outside (right) a forward-going cone of half-angle $40^{\circ}$ changes the relative spectra for different event types [11]. $\quad 20$

2.9 Energy resolution in ICARUS for low-energy electron based on MC studies. Only the ionization induced by the electron is considered for both reconstructed and truth information [33]. . . . . . . . . . . . . . . . . . . . . . . . . 21

3.1 Stopping power for muons in copper as a function of energy and momentum [39, 40]. 25 3.2 Skewed Landau energy loss probability distribution for pions traversing silicon [39]. 26 
3.3 The mean electronic stopping power $\langle d E / d x\rangle$ and the most probable electronic stopping power $\Delta_{p} / d x$ (or MPV) for several particle types traversing a $4-\mathrm{mm}$ length of LAr. . . . . . . . . . . . . . . . . . . . . . . . . . . . . . 27

3.4 Schematic diagram illustrating the production of observable quantities of charge $\left(e^{-}\right)$and scintillation $(\gamma)$ for an energy deposition in liquid argon. . . . . . . . . 28

3.5 Recombination in LArIAT according to the Modified Box Model (using ArgoNeuT parameterization [58]) and the Birks Model (using ICARUS parameterization [55]). The downturn in the Modified Box curve at low- $d E / d x$ is a mathematical artifact of the equation and does not describe actual recombination physics. . . . . . . . . . . . . . . . . . . . . . 33

$3.6 \quad$ Average optical signals for different levels of $\mathrm{N}_{2}$ contamination [65]. . . . . . . . 36

4.1 A schematic overhead view of LArIAT's beamline and TPC in the Meson Center at the Fermilab Test Beam Facility. . . . . . . . . . . . . . . . . . . . . . . . . . 42

4.2 The fully-sealed LArIAT cryostat (left) and the TPC sitting inside the open inner cryostat (right). . . . . . . . . . . . . . . . . . . . . 43

4.3 Schematic representation of the three drift regions in the LArIAT TPC. . . . . . 44

4.4 A view of the TPB-coated foils lining the field cage walls. . . . . . . . . . . . . . 46

4.5 A cartoon schematic illustration of VUV light being wavelength-shifting along the TPB-coated reflecting foils. . . . . . . . . . . . . . . . . . . . . 46

4.6 The light collection system mounted to the inner flange of the cryostat's side port prior to installation. . . . . . . . . . . . . . . . . . . . . . . . 47

4.7 Voltage divider for the $2 "$-diameter ETL D757-KFL PMT. . . . . . . . . . . . . 48

4.8 Voltage divider for the 3"-diameter Hamamatsu R-11065. . . . . . . . . . . . . . 48

4.9 Custom SiPM readout board for the $6 \times 6 \mathrm{~mm}^{2}$ SensL MicroFB-60035. . . . . . . 50 4.10 Schematic board diagram for the SensL MicroFB-60035 chip with an on-board inverting transimpedance amplifier circuit using an OPA656 op-amp. . . . . . . 50 
4.11 Custom SiPM readout board for the $12 \times 12 \mathrm{~mm}^{2}$ Hamamatsu S11828-3344M MPPC array. . . . . . . . . . . . . . . . . . . . . . 51

4.12 Schematic board diagram for Hamamatsu S11828-3344M MPPC array with an on-board inverting transimpedance amplifier circuit using an OPA656 op-amp. . $\quad 51$

4.13 The ETL PMT illuminated under a UV lamp after application of TPB film.

Visible re-emission from TPB induced by impinging UV radiation causes it to glow. 52

4.14 The Meson Center beam supercycle. Cosmic triggers are active in the 24-second COSMICON window following each beam spill. . . . . . . . . . . . . . . . 53

4.15 Schematic diagram of the logic used to make two of the light-based trigger signals in LArIAT, LARSCINT and MICHEL [74] . . . . . . . . . . . . . . . . . . . . . 55

4.16 An oscilloscope screen capture demonstrating the trigger for a cosmic Michel decay candidate captured using the light-based trigger. . . . . . . . . . . . . . . 55

4.17 Raw data event of a stopping muon with its decay electron from the Michel electron light-based trigger. Vertical columns of pixels represents the raw signal collected on wires. The color scale indicates wire signal amplitude at each time-tick. 56

5.1 Positron energy distribution from decay-at-ret $\mu^{+}$at the Columbia University cyclotron at the Nevis laboratories (left) [77]. On the right is the energy spectrum for bound $\mu^{-}$decaying in-orbit. Data from the TWIST experiment (black points) are compared to theoretical curves with (solid red line) and without (dotted blue) radiative corrections. The dashed green line represents the electron spectrum from free $\mu^{+}$that stop and decay [82]. . . . . . . . . . . . . . . . . . . . . . . 58

5.2 The electronics response of the wires as a function of time (arbitrarily-normalized) from BNL SPICE simulation of the LArIAT electronics. . . . . . . . . . . . . . 61

5.3 Simplified representations of the field response function (top) for the induction (left) and collection (right) plane wires. The bottom plots show real detector signals in solid lines and the simulated signals in dotted lines [84]. . . . . . . . . 61 
5.4 (top) Wire signals from a stopping track, and two examples of Gaussian hits fit to identified regions of interest (middle and bottom) [84]. . . . . . . . . . . . . . 64

5.5 Cartoon illustrating the proximity-based clustering process. Charge hits are represented as black circles in a 2D space. Clustered hits are shown with a blue outline. The seed hit is identified by a green star. . . . . . . . . . . . . . . .

5.6 An example representation of the 2D Michel clustering procedure on the collection plane. In the top plot, wire hits are represented as black circles, and the smaller blue dots indicate hits that were included in the original combined $\mu+\mathrm{e}$ cluster. The green star marks the seed hit, while the red star marks the identified muon endpoint based on hit charge and linearity conditions described in the text. Hits included in the final Michel electron shower are circled in orange. . . . . . . . . 67

\begin{tabular}{|lll|l|l|l}
\hline 5.7 & Clustering examples on the collection plane. See Figure & 5.6 & for an explanation
\end{tabular} of symbols. Note that charge has been expressed in units of electrons $\left(e^{-}\right)$; the ADC-to-electron conversion factor is introduced in Chapter 6 . . . . . . . . . . . 68

5.8 Clustering examples on the collection plane. See Figure 5.6 for an explanation of symbols. Note that charge has been expressed in units of electrons $\left(e^{-}\right)$; the ADC-to-electron conversion factor is introduced in Chapter 6 . . . . . . . . . .

5.9 The difference in hit times between the collection and induction planes for hits identified as the muon endpoint in events where a muon-electron boundary was identified on both planes. The peak is fit to a Gaussian (red line). . . . . . . . . 70

5.10 Overshoot in the Hamamatsu (HMM) PMT average waveform from Run IIB. . . 72 5.11 Individual exponential fits to the overshoot in average HMM PMT waveforms for all 9 amplitude ranges. The blue line shows the original averaged waveform, and the green line shows the waveform after the overshoot correction [87]. . . . . . . 73 5.12 Linear fit of $50 \mathrm{~ns}$ pulse integrals (integrated between $t=-5 \mathrm{~ns}$ and $t=45 \mathrm{~ns}$ ) to the undershoot normalization $A$ extracted from each fit [87]. . . . . . . . . . 73 
5.13 Tagged regions of a sample waveform to be used in the calculation of the running baseline (left), and the resulting running baseline for an example waveform shown (right). . . . . . . . . . . . . . . . . . . . . . 74

5.14 An inverted ETL PMT waveform with a zoomed-in view of the Michel candidate pulse for an event with decay time $\Delta T \approx 2.5 \mu \mathrm{s}$. The violet trace in the bottom plot is the signal gradient, with a dotted-red line signifying the identified location of the hit. The solid red line overlaid on the PMT signal plot is the estimated (fitted) muon late-light component which is subtracted off in the integration of the Michel pulse. . . . . . . . . . . . . . . . . . . . . . . . . . . . . 76

5.15 Fits to the average waveforms of crossing muon events. First the region $0.4-2 \mu \mathrm{s}$ is fit to a single exponential to extract $\tau_{\text {meas. }}$. Then, Equation $\mid$ A.1 $\mid$ is used to estimate the true quenched triplet lifetime $\tau_{t}^{\prime}$. A fit to $S_{\text {late }}(t)$ from Equation 5.8 is then performed over a larger time range, $0.4-7 \mu \mathrm{s}$, with parameter $\tau_{t}^{\prime}$ fixed. The ratio of the integral of the second component of the fit to the integral of the first component is computed and used to constrain the normalization parameter $B$ in fits during the late-light subtraction procedure in reconstruction: $B=f A \tau_{t}^{\prime} / 3550$, where $f=I_{A} / I_{B} . \ldots \ldots \ldots \ldots \ldots$

5.16 Amplitude of Michel-candidate optical pulses plotted against the measured decay time for the Run I (left) and Run IIB (right) datasets. For Run I, events are accepted with amplitude $>80$ ADC (on the ETL PMT). For Run IIB, we similarly impose an amplitude cut of 130 ADC for the HMM PMT and 45 ADC for the ETL PMT. . . . . . . . . . . . . . . . . . . . . . 79

5.17 Muon decay time data for Run I (left) and Run IIB (right) datasets. In Run I, only one PMT was used. Without a second PMT to verify true scintillation pulses, the Run I data is more likely to contain accidental noise events that will warp our spectra. . . . . . . . . . . . . . . . . . . . . . . . 80

$6.1 \quad$ Electron lifetimes in LArIAT measured over different run periods. . . . . . . . . 83 
6.2 Distribution from data (left) of separations between the reconstructed endpoint of the stopping muon track and the 3D Michel boundary point identified through the custom clustering procedure, projected along the direction of the reconstructed 3D track. On the right is the difference (in MC) between the reconstructed and true muon endpoint, projected along the reconstructed track direction, for events passing the quality selection cut illustrated by the orange dashed lines on the left plot. . . . . . . . . . . . . . . . . . . . . . . . . . . . 85

6.3 Reconstructed $d Q / d x$ and residual range along selected stopping muon tracks.

Shown below this histogram are several example fits to $d Q / d x$ using a Landau convoluted with a Gaussian. . . . . . . . . . . . . . . . . . . . . . . . . . 86

6.4 Mean $d E / d x$ versus residual range for muons. Points are taken from the Particle Data Group (PDG) table for muons in liquid argon [91]. The red curve is a

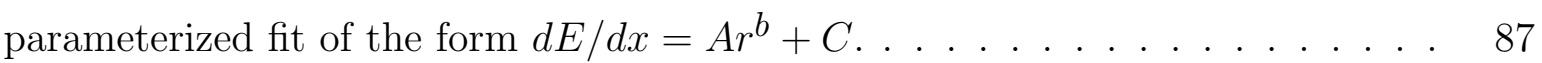

6.5 Charge response calibration fits for Run I and Run II using stopping muon tracks. 89

6.6 Examples of SPE calibration histograms for subsamples from the ETL PMT in Run I (top) and for both PMTs in Run IIB (bottom). . . . . . . . . . . . . . . . 91

6.7 On the left are plots of the SPE response measured over time for the Run I (top) and Run IIB Michel electron datasets. On the right, $\tau_{\text {meas }}$ extracted from fits to the average waveforms for both datasets. Vertical error bars in both SPE response and $\tau_{\text {meas }}$ plots represent the uncertainty on parameters returned by fits. 92

7.1 The calculated Rayleigh scattering length in liquid and solid argon as a function

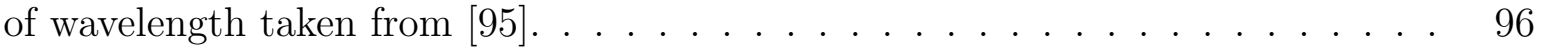

7.2 $\quad$ Projections of the average fractional scintillation visibility maps for Run I and IIB.

Note the differences in color-axis scale between the two runs, with $f_{\text {vis }}$ averaging at $\approx 0.02 \%$ in Run I (where only the ETL PMT is used) and five times higher, $\approx 0.1 \%$, in Run IIB (where both PMTs are operational). . . . . . . . . . . . . . 99 
7.3 True energy and deposited energy distributions for Michel electrons in the MC sample prior to any trigger efficiency cuts (left), and the energy containment fraction, $E_{d e p} / E$, plotted against the initial Michel electron energy $E$ (right). . . 100 7.4 The Birks recombination model (dashed line) and Modified Box recombination model (solid line) as functions of $d E / d x$ for two different electric field values. Parameterizations from fits to data from the ICARUS [55] and ArgoNeuT [58] experiments are used. An arbitrarily-normalized distribution of $d E / d x$ for Geant 4 particle steps is overlaid. . . . . . . . . . . . . . . . . . . . . . 101

7.5 Integral of baseline region used to estimate the inherent electronics noise in the Hamamatsu PMT for Run IIB. . . . . . . . . . . . . . . . . . . . . . . . . . 105

7.6 The effect of smearing contribution $\sigma_{X}$ on the total MC light distributions. . . . 108

7.7 The trigger efficiency function (red) overlaid with its respective prompt light distribution for PMTs in both MC samples. In each case the function is scaled arbitrarily and is drawn only for illustrative purposes. The unfilled distribution shows the prompt light distribution for all events in MC prior to trigger cuts. . . 108 7.8 Run I distributions of $S_{100}$ (left) and $S$ (right) compared to MC after smearing, scaling, and trigger efficiency parameters are optimized. . . . . . . . . . . . . . . 109

7.9 Run IIB distributions of $S_{100}$ (left) and $S$ (right) compared to MC after smearing, scaling, and trigger efficiency parameters are optimized. . . . . . . . . . . . . . . 109

8.1 Run I event cut distributions (top) and distribution of charge-weighted shower centroids (bottom). Selection boundaries indicated by dashed lines. . . . . . . . 117

8.2 Run I event cut distributions (top) and distribution of charge-weighted shower centroids (bottom). Selection boundaries indicated by dashed lines. . . . . . . . 118

8.3 Reconstructed distributions of free ionization electrons $(Q)$ and scintillation photons $(L)$ for the Run I Michel electron sample. . . . . . . . . . . . . . . . . . . . 119 8.4 Reconstructed distributions of free ionization electrons $(Q)$ and scintillation photons $(L)$ for the Run IIB Michel electron sample. . . . . . . . . . . . . . . . . . 119 
8.5 Reconstructed energy $E_{Q}^{\text {ion }}$ deposited by the portion of the event identified as the direct electron-induced ionization for Run I (left) and Run II (right). . . . . . . 121

8.6 Reconstructed energy of the full Michel electron shower for Run I (top) and Run II (bottom). Equation 8.1 is used to calculate the charge-based $E_{Q}$ (left), while Equation 8.2 is used to calculate energy by directly combining charge and light (right). . . . . . . . . . . . . . . . . . . . . . 123

8.7 Examples of reconstructed $Q$ (left) and $L$ (right) distributions for simulated Michel electrons depositing $30 \mathrm{MeV}$ in the LArIAT TPC. The red lines are fits to the distribution. For $Q$ the fit is to a double-Gaussian function, while $L$ is fit to a single Gaussian at all energies. The dotted orange line in $Q$ illustrates the "background" (BG) Gaussian in the double-Gaussian fit. . . . . . . . . . . . . . 125

8.8 Reconstructed $Q$ distributions for events of different deposited energies in the LArIAT TPC. Events are distributed randomly, so each distribution is effectively an average over all possible locations. The red line is a fit to a double-Gaussian function. The dotted orange line illustrates the secondary "background" (BG) Gaussian in the total fit. (Parameters: $N_{\text {peak }}=\mathrm{p} 0, \mu_{\text {peak }}=\mathrm{p} 1, \sigma_{\text {peak }}=\mathrm{p} 2$, $\left.N_{B G}=\mathrm{p} 3, \mu_{B G}=\mathrm{p} 4, \sigma_{B G}=\mathrm{p} 5.\right) \ldots \ldots \ldots \ldots \ldots \ldots$

8.9 Reconstructed $L$ distributions for events of different deposited energies in the LArIAT TPC. Events are distributed randomly, so each distribution is effectively an average over all possible locations. The red line is a fit to a Gaussian function.

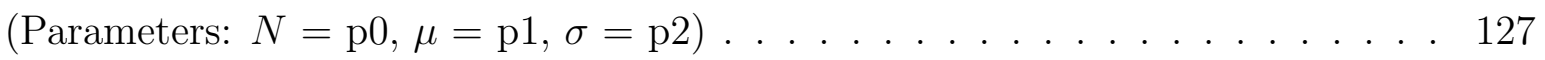
8.10 Parameters describing the charge PDF $f_{Q}$ (top) and light PDF $f_{L}$ (bottom) modeled as functions of energy deposited in the TPC by the Michel electron shower.

The equations for the fits to $f_{Q}$ parameters are outlined in Equations 8.10 8.14 while those for $f_{L}$ are in Equations $\mid 8.15 \| 8.16$. . . . . . . . . . . . . . . . . . . 129

8.11 Charge distributions overlaid with parameterizations. . . . . . . . . . . . . . . . 130

8.12 Light distributions overlaid with parameterizations. . . . . . . . . . . . . . . . 130 
8.13 A comparison of the Michel electron shower energy spectrum from Run IIB reconstructed using the the two simple formulaic constructions, $E_{Q}$ (left) and $E_{Q L}$ (middle), as well as the spectrum using the likelihood fitting technique, $E_{Q L}^{\text {likelihood }}$ (right).

8.14 The energy resolution of Michel electron events in LArIAT for energies reconstructed from charge (blue) as well as from both charge and light (violet and green). .

8.15 The energy resolution, as defined by Equation 8.18 , of isolated electron showers in the LArIAT TPC under Run II operating conditions. . . . . . . . . . . . . . . 134

9.1 Energy resolution for the electron ionization (excluding photon deposits) for the three different simulated samples. . . . . . . . . . . . . . . . . . . . . . . . . . . 140

9.2 The fractional improvement in resolution with a signal-to-noise of $\mathrm{S} / \mathrm{N} \approx 10$ for the four simulated light yields. . . . . . . . . . . . . . . . . . . . . . . . . . . . 140

9.3 Energy resolution for charge-only energy $\left(E_{Q}\right)$ and the light-augmented likelihood energy $\left(E_{Q L}^{\text {likelihood }}\right)$ for different wire noise levels and light yields. Fits are performed to function $\sigma=A / \sqrt{E} \oplus B$. . . . . . . . . . . . . . . . . . . . . . 141

A.1 Fitted linear relationship between the true quenched triplet lifetime and the measured lifetime found from fitting a single exponential to the resulting optical waveform from $0.4-2 \mu \mathrm{s}$.

B.1 Fits to the energy resolution for charge-based energy $\left(E_{Q}\right)$ of Michel electron events in LArIAT. . . . . . . . . . . . . . . . . . . . . . . . . . . . . . . . . . 149

B.2 Fits to the energy resolution for combined charge and light energy $\left(E_{Q L}\right)$ of Michel electron events in LArIAT. . . . . . . . . . . . . . . . . . . . . . . . . . . 150

B.3 Fits to the energy resolution using the maximum likelihood method $\left(E_{Q L}^{\text {likelihood }}\right)$ for Michel electron events in LArIAT. . . . . . . . . . . . . . . . . . . . . . . . . 151 
B.4 Gaussian fits to $Q$ distributions for isolated electron showers for nominal LArIAT conditions at different energies. . . . . . . . . . . . . . . . . . . . . . . . . . . . 152

B.5 Gaussian fits to $L$ distributions for isolated electron showers for nominal LArIAT conditions at different energies. . . . . . . . . . . . . . . . . . . . . . . . . . . . 152

B.6 Gaussian fits to the charge-only $E_{Q}$ energy variance $(\delta E)$ for isolated electron showers for nominal LArIAT conditions at different energies. . . . . . . . . . . . 153

B.7 Gaussian fits to $E_{Q L}$ energy variance $(\delta E)$ for isolated electron showers for nominal LArIAT conditions at different energies. . . . . . . . . . . . . . . . . . . . . 154

B.8 Gaussian fits to the maximum-likelihood energy $E_{Q L}^{\text {likelihood }}$ energy variance $(\delta E)$ for isolated electron showers for nominal LArIAT conditions at different energies. 155

B.9 Fits to energy variance $(\delta E)$ for the electron-induced ionization track $\left(E^{\text {ion }}\right)$ for nominal LArIAT conditions at different energies. . . . . . . . . . . . . . . . . . 156

B.10 Gaussian fits to the energy resolution for isolated electron showers at a light yield

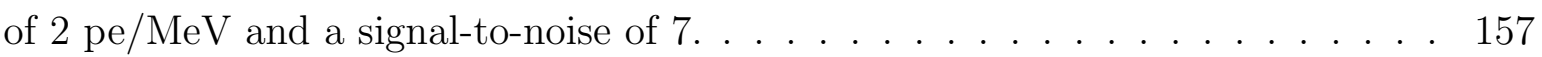

B.11 Gaussian fits to the energy resolution for isolated electron showers at a light yield of $10 \mathrm{pe} / \mathrm{MeV}$ and a signal-to-noise of 10.

B.12 Gaussian fits to the energy resolution for isolated electron showers at a light yield of $20 \mathrm{pe} / \mathrm{MeV}$ and a signal-to-noise of $70 . \quad \ldots \ldots \ldots$ 


\section{List of Tables}

2.1 Existing measurements of the three neutrino mixing angles, for a normal mass hierarchy, taken from a global analysis of oscillation results done in [18]. Here, $\Delta m^{2}=m_{3}^{2}-\left(m_{1}^{2}+m_{2}^{2}\right) / 2$, which is approximately equal to $\Delta m_{13}^{2}$ and $\Delta m_{23}^{2} . \quad 11$

$3.1 \quad$ Physical properties of liquid $\operatorname{argon}(\mathrm{LAr}) . \quad \ldots \ldots \ldots$

5.1 Summary of the nominal data samples used in this analysis. . . . . . . . . . . . 59

5.2 Summary of results for the negative muon lifetime. . . . . . . . . . . . . . . . 81

$7.1 \quad$ Relevant parameters used in the standalone photon propagation Monte Carlo. . 97

7.2 Measured TPB decay times and relative abundances (adapted from [67]). In the simulation, the instantaneous component (1-10 ns) is modeled as $\tau=5 \pm 5 \mathrm{~ns}$. . 103

7.3 Smearing, scaling, and trigger-cut parameters applied to the MC samples. Also displayed are the data-MC $\chi_{w}^{2}$ comparison values after minimization for both prompt $\left(S_{100}\right)$ and total $(S)$ photoelectrons. . . . . . . . . . . . . . . . . . 107

$8.1 \quad$ Event reduction table for all data and MC samples. . . . . . . . . . . . . . . . . 116

8.2 Average light yield (LY) in units of photoelectrons per MeV (pe/MeV). . . . . . 122

8.3 The largest sources of systematic error on calorimetric reconstruction. . . . . . . 138 


\section{Acknowledgments}

I would first like to acknowledge my thesis advisor, David Schmitz. I can't express my gratitude enough for the opportunities you've given me. When things got murky, you were always able to restore the clarity and confidence needed to keep moving forward. Thank you for your advice, your encouragement, and for always challenging me to go one step further.

Many thanks to my thesis committee - Ed Blucher, Scott Wakely, and Carlos Wagner. This analysis would not have been as impactful without your guidance and suggestions.

To Andrzej Szelc, thank you for taking me under your wing those first few years. I'll never forget frantically wheeling liquid nitrogen across campus in the dead of night to keep the PMT tests running smoothly. It's been a fun journey; I'm glad you were a part of it.

Jen Raaf and Jonathan Asaadi - I've felt so incredibly welcomed into this collaboration since the very first day. Thank you for fostering such a supportive environment, and for helping to bring these analyses to fruition. And to Flavio Cavanna - your convivial spirit and endless expertise have been hugely influential. Thank you especially to all those who made LArIAT the fun and unique experience it was - Jason, Elena, Paweł, and Greg among so many others. It's been an absolute pleasure working with you all.

I've been lucky to work with a fantastic group of people at UChicago. To Johnny Ho, thank you for being such a great friend and labmate. Our crazy car-ride conversations to and from Fermilab will always stick with me. To my first advisor, Young-Kee Kim: your limitless energy and enthusiasm continue to inspire me. Special shout-outs to Mark Zaskowski in the e-shop for the generous soldering assistance, and to Mary Heintz for solving countless technical problems with NORAD-level responsiveness.

I'm so grateful for the friends I've made here in Chicago - especially Nate, Ravi, Zach, Barry, Joakim, Yangyang, Lisa, and Daniel. You've helped make this city feel like home.

Finally, to my parents, Faye and Mike - I'm so privileged to be where I am today, and that's due in no small part to your love and support. You've given me so much. Thank you. 


\section{Abstract}

The ability to resolve electrons with energies as low as $5 \mathrm{MeV}$ in liquid argon (LAr) is important for future studies of astrophysical neutrinos in liquid argon time projection chambers (LArTPCs). Existing methods for measuring the energy of electromagnetic showers in LArTPCs rely solely on charge-based reconstruction which requires correcting for assumed electron-ion recombination. However, LAr scintillation light has the potential to improve energy reconstruction over charge-based measurements alone. Here we present a demonstration of a light-augmented calorimetric technique for low-energy electrons in LAr that foregoes the need to correct for recombination. A sample of Michel electrons from stopping cosmic muons is collected in LArIAT (Liquid Argon In A Testbeam), a single-phase LArTPC at Fermilab's Test Beam Facility. Michel electron energy spectra are reconstructed using both a traditional charge-based approach as well as a more holistic approach that incorporates both charge and light. A maximum-likelihood fitter, using LArIAT's well-tuned simulation, is developed for combining these quantities to achieve optimal energy resolution. A sample of isolated electrons with $E_{e}<60 \mathrm{MeV}$ is then simulated to better determine the energy resolution of this topology. Due to LArIAT's low wire noise, the addition of light is not found to significantly improve upon the achieved charged-based resolution of $\sigma / E \simeq 9 \% / \sqrt{E} \oplus 1 \%$. However, additional samples are generated with varying wire noise levels and light yields to gauge the impact of light-augmented calorimetry in large LArTPCs such as DUNE and SBND. At a charge-readout signal-to-noise of $\approx 10$, the energy resolution for low-energy electrons is improved by $\sim 15 \%, \sim 25 \%$, and $\sim 45 \%$ over charge-only calorimetry for average light yields of 10,20 , and $100 \mathrm{pe} / \mathrm{MeV}$, respectively. 


\section{1}

\section{Introduction}

"Nature has conservation of energy, but does not really care; she spends a lot of it in all directions."

- Richard Feynmann, Six Easy Pieces

About $10^{38}$ neutrinos are born in the Sun's core every second, nearly every one destined to stream across the Universe for billions of years unimpeded. Only about one out of every two billion will pass through the Earth on its way out of the solar system, a vast majority of them flying through the planet as if it weren't there. In fact, neutrinos are produced all across the Universe. We are bathed in them; 65 billion per second pass through every square centimeter. They come from burning stars, energetic particle interactions, and from every source of radioactivity, including the rock and soil we stand upon. Some come from supernova explosions, and some are remnants from the Big Bang itself. In the words of Frank Close, "if we could see with neutrino eyes, night would be as bright as day" [1.

The idea of the neutrino $(\nu)$ - a "desperate remedy" to explain the continuous energy spectrum of electrons from radioactive decay - was first put forth in 1930 by Wolfgang Pauli. Enrico Fermi built upon this idea several years later with his theory of beta decay in which the emission of an electron-neutrino pair from a decaying nucleus was modeled following the same framework used to describe the emission of photons by excited atoms [2]. It wasn't until 1956 that the first experimental evidence confirming the existence of the neutrino was produced by Frederick Reines and Clyde L. Cowan, Jr. To accomplish this, Reines and Cowan used two 200-liter tanks filled with water to detect the time-separated scintillation light signals produced by the annihilation of the outgoing positron and the capture of the outgoing neutron from inverse beta-decay interactions of neutrinos (or more specifically, antineutrinos, $\bar{\nu}$ ) emitted by a nuclear reactor at Savannah River, South Dakota [3].

We have learned a substantial amount about neutrinos since their discovery over 60 years 
ago. Experiments have shown they oscillate between flavors, a beautiful discovery which by itself implies they have mass and thus defies the Standard Model as we know it. Their mass differences and the mixing angles that parameterize the mass eigenstate content of each flavor-state have been measured [4]. However, despite their being the second-most abundant elementary particle in the Universe, neutrinos still remain the least-understood in the Standard Model of particle physics. We still don't know their absolute mass scale, relative mass ordering, nor whether this mixing is asymmetric between neutrinos and antineutrinos, a finding that would indicate a new source of charge-parity $(\mathrm{CP})$ violation in particle physics. There are even hints of possible new physics in the neutrino sector such as the existence of heavier "sterile" states that don't interact through the weak force [5, 6].

The next generation of experiments centered at Fermilab will unravel many of these puzzles by measuring oscillations of accelerator neutrinos over both short and long distances. The liquid argon time projection chamber (LArTPC) is the technology of choice in this new era of neutrino research. These detectors are ideal for reconstructing neutrino final-state topologies given their precise 3D particle tracking, calorimetric capabilities, and scalability to large active volumes. The Short-Baseline Neutrino (SBN) Program, which will measure neutrino cross-sections and oscillations along the Booster beamline over a baseline of $\approx 1 \mathrm{~km}$, is presently underway with MicroBooNE already running and the Short-Baseline Near Detector (SBND) and ICARUS far detector soon to be installed [7]. The Deep Underground Neutrino Experiment (DUNE), a massive LArTPC to be built over the next decade, will make precision neutrino oscillation measurements for neutrinos traveling $1300 \mathrm{~km}$ through the Earth [8, 9].

DUNE's large active volume will also enable the detection of neutrinos from the Sun [1] and - if we are lucky - from galactic core-collapse supernovae [12]. Of these low-energy astrophysical neutrinos, LAr is most sensitive to interactions of the electron-type $\left(\nu_{e}\right)$. These events will feature an electron on the order of $10 \mathrm{~s}$ of $\mathrm{MeV}$ or less emanating from the neutrino interaction point. This energy will go into ionizing and exciting Ar atoms which, 
through recombination and de-excitation channels, will ultimately result in the production of detectable quantities of free electrons (charge) and scintillation photons (light) in roughly equal amounts. Traditionally, only the collected charge is used to perform calorimetric measurements, but the addition of light has the potential to improve energy resolution.

This thesis presents results from the LArIAT (Liquid Argon In A Testbeam) Experiment at Fermilab in which light and charge are combined to reconstruct the energies of electrons. LArIAT is a 170-liter active volume LArTPC that operated in the Fermilab Test Beam Facility from 2015 to 2017. The unique design of LArIAT's light detection system allowed it to collect a much larger fraction of generated scintillation light compared to previous LArTPCs. This light allowed us to engineer a trigger on the delayed decay electrons from cosmic muons that come to rest in the TPC volume during the dedicated cosmic readout period following every beam spill. Both charge and light from these electron events are reconstructed and used in calorimetry to achieve an energy resolution that improves upon that from charge-only energy measurements.

First, in Chapter 2, the current state of neutrino physics is reviewed, and ongoing efforts to use LArTPCs to answer open questions in the field are used to motivate the need for improved calorimetry at low energies. Chapter 3 discusses the physics of energy deposition by charged particles in LAr and how this energy is divided into observable quantities of charge and light. The LArIAT Experiment and its light collection system is introduced in Chapter 4. In Chapter 5, the Michel electron sample is introduced and Michel-specific reconstruction needed to extract information about the charge and light deposited by the decay electrons is described. The reconstructed muon decay time spectrum is also presented in order to validate the sample through literature-compatible measurements of the negative muon capture lifetime and the cosmic muon charge ratio. Calibrations needed to correct the measurements of charge and light - including response calibrations for the wires and photodetectors as well as electron attenuation lifetime measurements to compensate for charge lost during the drift - are explained in Chapter 6. The Monte Carlo (MC) simulation 
of the LArIAT detector is described in Chapter 7. In Chapter 8, the corrected charge and light measurements are finally presented and used to produce Michel electron energy spectra, which are compared to MC. The MC is used to evaluate the energy resolution in LArIAT. These MC studies are expanded in Chapter 9 to make projections of the achievable energy resolution of isolated low-energy electron showers in larger LArTPCs like DUNE. Finally, some concluding remarks are offered in Chapter 10.

The LArTPC is still a relatively young technology in neutrino physics, and reconstruction techniques are under active development. With the work presented here, we hope to contribute to efforts in maximizing the effectiveness of these detectors - pushing them to their limits to extract as much physics as possible from the data they have to offer. 


\title{
2
}

\section{Detecting Neutrinos with Liquid Argon TPCs}

\author{
"Nature does not hurry, yet everything is accomplished."
}

- Lao Tzu

This chapter reviews our present understanding of neutrino physics and how it is motivating new experimental efforts using liquid argon TPCs, setting the stage for all that follows. In Section 2.1, a theoretical overview of the physics underlying the existence of neutrinos within the Standard Model is presented along with the phenomena of neutrino oscillations and its implications. Section 2.2 discusses the puzzles about neutrino behavior that remain to be solved, while Section 2.3 introduces the LArTPC technology behind the experiments underway or being planned to address some of these puzzles. In Section 2.4 we discuss some of the more exotic studies that would be possible in a large underground LArTPC, including the detection of neutrinos from astrophysical sources. Finally in Section 2.5, we lay out the importance of low-energy electron reconstruction capabilities in such a LArTPC and present results from previous experiments that have attempted to reconstruct this topology in LAr.

\subsection{Neutrino physics: what we know}

The Standard Model (SM) is an effective quantum field theory that describes the strong, weak, and electromagnetic interactions. It is constructed from boson (integer spin) and fermion ( $\left.\operatorname{spin}-\frac{1}{2}\right)$ fields that pervade all of space, governed by a local $S U(3) \times S U(2) \times U(1)$ gauge symmetry. Figure 2.1 illustrates the full set of particles comprising the SM.

Neutrinos are among the spin- $\frac{1}{2}$ fermions in the SM known as leptons. They arise naturally as solutions to the massless $(m=0)$ Dirac equation [13],

$$
i \gamma^{\mu} \psi=0,
$$




\section{Standard Model of Elementary Particles}

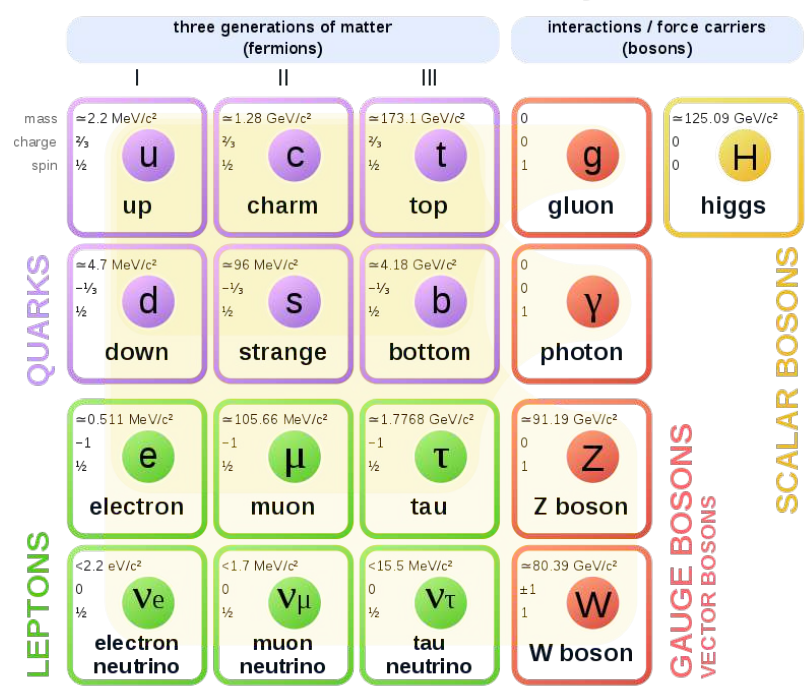

Figure 2.1: The fundamental particles of nature as described by the Standard Model [14].

which divides into two decoupled equations of two-component spinors describing left-handed $\left(\nu_{L}\right.$ and $\left.\overline{\nu_{R}}\right)$ and right-handed $\left(\nu_{R}\right.$ and $\left.\overline{\nu_{L}}\right)$ neutrino fields. Because neutrinos are presumed to interact only through the weak force, they lack any color or electrical charge. Further, due to the vector-axial $(V-A)$ structure of the weak current, which maximally violates parity by acting only on left-handed states (famously confirmed through the observation of outgoing $\beta$ from polarized cobalt nuclei by $\mathrm{Wu}$ in 1956 [15]), it is only $\nu_{L}\left(\right.$ or $\overline{\nu_{R}}$ ) which can interact with charged leptons. This leaves any hypothetical $\nu_{R}$ states unobservable [13]. The fermions of the SM can therefore be described by six left-handed isodoublets and nine right-handed isosinglets:

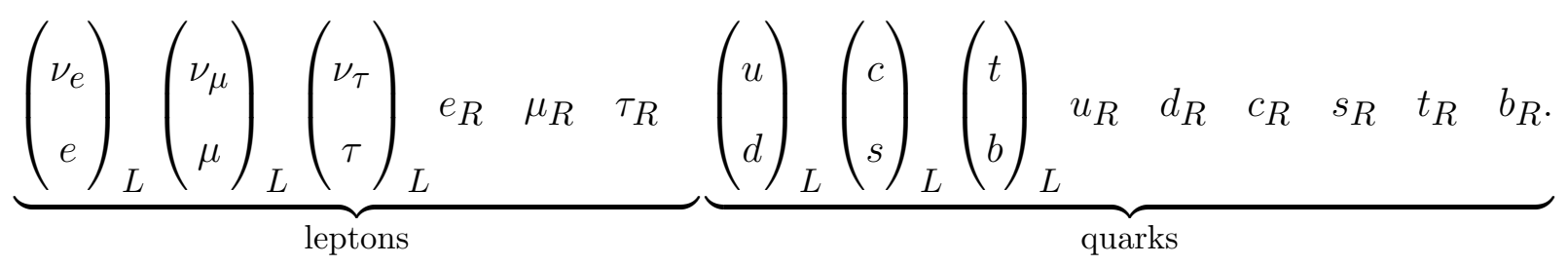

Aside from neutrinos, all the remaining fermions acquire mass in the SM through spontaneous symmetry breaking via the Higgs mechanism, which relies on a coupling between the left- and right-handed fields [13]. However, experiments have revealed that at least some 
of the neutrinos do, in fact, have mass [16]. The SM can be extended to accommodate massive neutrinos (in a way that preserves the conservation of lepton number exhibited in the unmodified SM) through the addition of a "Dirac mass term", $\mathcal{L}_{\mathcal{D}}$, to the SM Lagrangian:

$$
\mathcal{L}_{D}=-m_{D} \overline{\nu_{L}} \nu_{R}+\text { h.c. }
$$

where $m_{D}$ is a constant and $\nu_{R}$ is the right-handed neutrino field that is omitted in the SM but must be added to generate mass [17]. However, neutrino mass can also be generated in a Lorentz-invariant way that requires only one of the neutrino fields, though this would require that the neutrino is its own antiparticle and lepton number is not conserved. If these are true, then the SM Lagrangian allows for "Majorana mass terms" of the form

$$
L_{M}=-m_{M} \bar{\nu}^{C} \nu+\text { h.c. }
$$

where $\nu^{C}$ is the charge-conjugate of the neutrino $\nu$. As of now, it is unknown whether neutrinos are their own antiparticle (i.e., Majorana particles). Experiments looking for neutrinoless double- $\beta$ decay will help determine whether or not this is the case.

Neutrinos react with other particles exclusively through the weak force either by the exchange of a charged $W^{+/-}$or neutral $Z$ boson - referred to as charged-current (CC) and neutral-current (NC) interactions, respectively. In $\mathrm{NC}$ interactions of neutrinos and free nucleons $(N)$, the incoming $\nu$ effectively scatters off the nucleon, $\nu N \rightarrow \nu N$, and the final-state consists of an outgoing (invisible) $\nu$ as well as a recoiling $N$. However, for $\mathrm{CC}$ interactions off of protons or neutrons ( $p$ or $n)$, an outgoing charged lepton $(\ell)$ of the same flavor-type as the incoming neutrino or antineutrinos is produced in the final state: $\nu_{\alpha} n \rightarrow \ell_{\alpha}^{-} p$, or $\bar{\nu}_{\alpha} n \rightarrow \ell_{\alpha}^{+}$.

Because neutrinos interact only through the weak force and gravity, we can only probe their weak flavor states $(e, \mu, \tau)$. We know neutrinos have non-zero masses from the experimental confirmation of a phenomenon called "neutrino oscillations" in which a neutrino 
changes its flavor-state over time as it propagates [16]. In other words, a neutrino born of flavor $\alpha$ will, after traveling through some distance, behave as if it were of a different flavor $\beta$, thus producing an outgoing $\beta$ lepton in a $\mathrm{CC}$ interaction. Since it is the mass eigenstates that govern the time-evolution of a neutrino, the fact that flavor-state oscillations occur implies that the mass eigenstates $\left(\left|\nu_{i}\right\rangle\right)$ and weak flavor eigenstates $\left(\left|\nu_{\alpha}\right\rangle\right)$ are not the same. A neutrino of flavor $\alpha$ is thus a quantum superposition of the mass eigenstates,

$$
\left|\nu_{\alpha}\right\rangle=\sum_{i} U_{\alpha i}^{*}\left|\nu_{i}\right\rangle
$$

where $U$ is a unitary leptonic mixing matrix. Most experimental results point to there being three mass eigenstates $(i=1,2,3)$, suggesting $U$ is a $3 \times 3$ matrix. The transformation between the flavor basis to the mass eigenstate basis is carried out using a form of this matrix called the Pontecorvo-Maki-Nakagawa-Sakata matrix $\left(U_{P M N S}\right)$ :

$$
\left(\begin{array}{c}
\nu_{e} \\
\nu_{\mu} \\
\nu_{\tau}
\end{array}\right)=\underbrace{\left[\begin{array}{ccc}
U_{e 1} & U_{e 2} & U_{e 3} \\
U_{\mu 1} & U_{\mu 2} & U_{\mu 3} \\
U_{\tau 1} & U_{\tau 2} & U_{\tau 3}
\end{array}\right]}_{U_{P M N S}} \times\left(\begin{array}{c}
\nu_{1} \\
\nu_{2} \\
\nu_{3}
\end{array}\right)
$$

The matrix $U_{P M N S}$ can be written as

$$
U_{P M N S}=\underbrace{\left[\begin{array}{ccc}
c_{12} & s_{12} & 0 \\
-s_{12} & c_{12} & 0 \\
0 & 0 & 1
\end{array}\right]}_{\text {solar }} \underbrace{\left[\begin{array}{ccc}
c_{13} & 0 & s_{13} e^{-i \delta} \\
0 & 1 & 0 \\
-s_{13} e^{i \delta} & 0 & c_{13}
\end{array}\right]}_{\text {cross-mixing/reactor }} \underbrace{\left[\begin{array}{ccc}
1 & 0 & 0 \\
0 & c_{23} & s_{23} \\
0 & -s_{23} & c_{23}
\end{array}\right]}_{\text {atmospheric }} \text {, }
$$

where $c_{i j}=\cos \theta_{i j}$ and $s_{i j}=\sin \theta_{i j}$. In this representation, $\theta_{i j}$ are "mixing angles" and $\delta$ is a CP-violating phase 1 . The three quantum mass eigenstates interfere constructively

1. Here we have excluded any CP-violating phases one would expect if neutrinos were Majorana particles. 
or destructively as the neutrino propagates, resulting in flavor-state oscillations that are dependent on the length of travel $(L)$. Given this, the amplitude for a neutrino of flavor $\alpha$ of energy $E$ to change to flavor $\beta$ is given by

$$
\psi_{\nu_{\alpha} \rightarrow \nu_{\beta}}=\sum_{i} U_{\alpha i}^{*} e^{-i m_{i}^{2} L /(2 E)} U_{\beta i}
$$

Squaring this amplitude, we arrive at the probability of oscillation [17],

$$
\begin{aligned}
\left|\psi_{\nu_{\alpha} \rightarrow \nu_{\beta}}\right|^{2}=P_{\nu_{\alpha} \rightarrow \nu_{\beta}}=\delta_{\alpha \beta} & -4 \sum_{i>j} \mathcal{R}\left[U_{\alpha i}^{*} U_{\beta i} U_{\alpha j} U_{\beta j}^{*}\right] \sin ^{2}\left(\Delta m_{i j}^{2} \frac{L}{4 E}\right) \\
& +2 \sum_{i>j} \mathcal{I}\left[U_{\alpha i}^{*} U_{\beta i} U_{\alpha j} U_{\beta j}^{*}\right] \sin \left(\Delta m_{i j}^{2} \frac{L}{2 E}\right)
\end{aligned}
$$

where $\Delta m_{i j}^{2}=m_{i}^{2}-m_{j}^{2}$. We see that the oscillation probability is a function of $L / E$ and that the mass splittings $\Delta m_{i j}$ must be non-zero.

For a beam of neutrinos of known flavor $\nu_{\alpha}$, oscillations can be probed by looking for either the appearance of $\nu_{\beta}\left(P_{\mathrm{app}}=P_{\nu_{\alpha} \rightarrow \nu_{\beta}}\right)$, or for the disappearance of $\nu_{\alpha}\left(P_{\text {disapp }}=\right.$ $\left.1-P_{\nu_{\alpha} \rightarrow \nu_{\alpha}}\right)$ assuming the beam flux is known to sufficient precision. Experimentalists often simplify the oscillation probability formula by assuming that the mass splittings between the different mass eigenstates are vastly different in scale - i.e., $\Delta m_{12}^{2} \ll \Delta m_{23}^{2}$-implying highly disparate oscillation length scales. Under this assumption, only one splitting is important for any given experiment, and the oscillation probability can be approximated as a function of the mixing angle $\theta$ associated with the relevant mass states dominating the oscillation,

$$
P_{\nu_{\alpha} \rightarrow \nu_{\beta}}=\sin ^{2}(2 \theta) \sin ^{2}\left(1.27 \Delta m^{2}\left[\mathrm{eV}^{2}\right] \frac{L[\mathrm{~km}]}{E[\mathrm{GeV}]}\right)
$$

where the previously-omitted factors ' $\hbar$ ' and ' $c$ ' have been added back in and absorbed into the numerical factor in the sin-squared argument. It is clear from Equation 2.9 that an experiment's ability to measure an oscillation pattern is a function of the ratio of its 


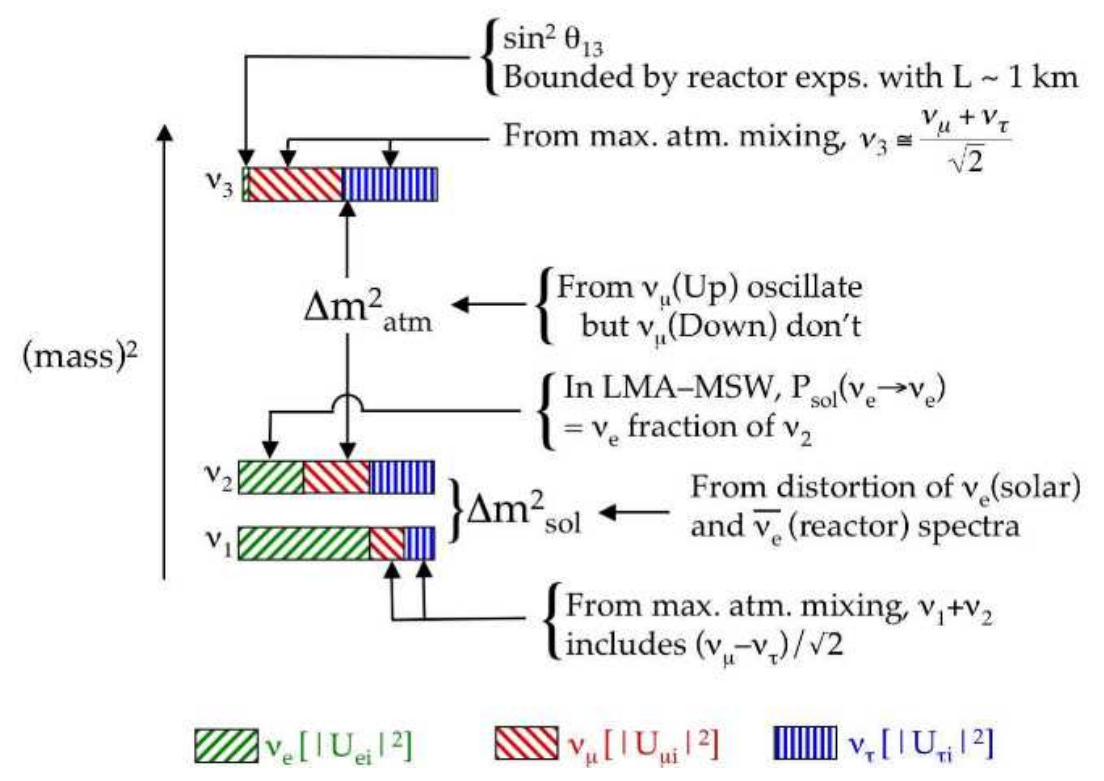

Figure 2.2: A schematic of the neutrino squared-mass spectrum assuming three mass eigenstates and a "normal" mass-ordering. The flavor content of each mass state is illustrated based on existing experimental results (excluding those from LSND) [17].

oscillation baseline to the neutrino energy, $L / E$, as well as the magnitude of the mass splitting. For comparable $E$, it follows that experiments using shorter baselines are more sensitive to oscillations dominated by high- $\Delta m^{2}$ splittings, whereas longer baselines will more effectively probe oscillations from small $\Delta m^{2}$.

It is illuminating to decompose mixing matrix $U_{P M N S}$ into three component matrices as we've done in Equation 2.6 because it highlights the different oscillation length scales and thus the nature of the experiments that measured their mixing angles. Figure 2.2 illustrates the three-neutrino mass spectrum for a normal mass ordering $\left(m_{1}<m_{2}<m_{3}\right)$ as well as the inferred flavor content of each mass state, while Table 2.1 summarizes existing neutrino mixing angle and mass splitting measurements.

The oscillation probabilities presented so far have been computed for neutrinos traveling through vacuum. In reality, the presence of matter will slightly alter these derived results. This comes about via the possibility of coherent forward scattering of $\nu_{e}$ through $W^{ \pm}$-boson exchange with bound electrons in atoms, which adds an extra interaction potential energy to the $\nu_{e}$. For electron-type antineutrinos, $\bar{\nu}_{e}$, the potential energy term has the same 
Table 2.1: Existing measurements of the three neutrino mixing angles, for a normal mass hierarchy, taken from a global analysis of oscillation results done in [18]. Here, $\Delta m^{2}=$ $m_{3}^{2}-\left(m_{1}^{2}+m_{2}^{2}\right) / 2$, which is approximately equal to $\Delta m_{13}^{2}$ and $\Delta m_{23}^{2}$.

\begin{tabular}{|cc|l|}
\hline \multicolumn{2}{|l|}{ Parameter } & Value \\
\hline$\theta_{12}$ & ("solar mixing angle") & $33_{-11}^{+10}$ degrees \\
$\Delta m_{12}^{2}$ & $\left(\approx \Delta m_{\text {sol }}^{2}\right)$ & $7.37_{-0.16}^{+0.17} \times 10^{-5} \mathrm{eV}^{2}$ \\
\hline$\theta_{23}$ & ("atmospheric mixing angle") & $41.4_{-1.2}^{+1.9}$ degrees \\
$\Delta m^{2}$ & $\left(\approx \Delta m_{13}^{2} \approx \Delta m_{23}^{2} \approx \Delta m_{\text {atm }}^{2}\right)$ & $2.50 \pm 0.04 \times 10^{-3} \mathrm{eV}^{2}$ \\
\hline$\theta_{13}$ & ("reactor mixing angle") & $8.4 \pm 0.2$ degrees \\
\hline
\end{tabular}

magnitude but with opposite sign. These contributions raise the effective mass of $\nu_{e}$ in matter while reducing the effective mass for $\bar{\nu}_{e}[17]$.

\subsection{Open questions in neutrino physics}

Despite all that has been learned about neutrinos, there remain many open questions that current and future experiments will address. The most prominent ones that can be answered through accelerator-based neutrino oscillation experiments, which are most relevant to topics addressed in this thesis, are outlined below.

- How many neutrino species are there? Results from the Liquid Scintillator Neutrino Detector (LSND), which looked for $\bar{\nu}_{e}$ in a beam of $\nu_{\mu}$ over a short baseline, showed an excess of detected $\bar{\nu}_{e}$ compared to expectations [5]. The best-fit mass splitting, $\Delta m_{L S N D}^{2}=1.2 \mathrm{eV}^{2}$, cannot be explained by the extended 3- $\nu \mathrm{SM}$ described previously in this chapter. The MiniBooNE Experiment at Fermilab reported a similar excess of electron-like neutrino events, though the background was dominated by photon-induced showers from $\mathrm{NC}$ events which were misidentified as electrons [6]. If such excesses are to be explained by neutrino oscillations, and not by a misidentification of background, clearly the $3-\nu$ model is insufficient and new mass eigenstates must be proposed. Due to 
constraints from LEP at CERN [19] on the total number of weakly-interacting neutrino species $\left(N_{\nu}=2.984 \pm 0.008\right)$, any new massive neutrinos would not interact through the weak force and would be termed "sterile."

- Do neutrinos violate charge-parity $(C P)$ conservation? If equal amounts of matter and antimatter were produced by the Big Bang as conservation and symmetry arguments suggest, then the presently-observed dominance of matter over antimatter must have arisen due to some CP-violating physical process in which particles and their antimatter partners behave differently. While CP-violation has been observed in quark interactions, as predicted in the SM, the effect is too small to explain the matterdominated Universe in which we find ourselves. The discovery of leptonic CP-violation - for example, an observed difference in oscillation behavior between $\nu$ and $\bar{\nu}$ - could help explain this imbalance if the effect is sufficiently significant. One natural way this can come about is through what is known as the see-saw mechanism in which the interplay between Dirac mass terms and the mass term of heavy right-handed Majorana neutrinos $N$ of mass $M$ results in an effective mass term for the left-handed neutrinos which is inversely proportional to $M$. Such heavy neutrinos would have been produced in the Big Bang and subsequently decayed to charged leptons as the Universe cooled. If the decay rate $\Gamma$ is different between these heavy neutrinos and their antiparticles $\left(\Gamma\left[N \rightarrow \ell^{+} \ldots\right] \neq \Gamma\left[N \rightarrow \ell^{-} \ldots\right]\right)$, the resulting imbalance in leptons and antileptons would have led to a corresponding imbalance in the baryons as well through sphaleron processes [16, 17]. An observation of non-zero $\delta$ in the PMNS matrix would be a signature of leptonic CP-violation.

- How are the neutrino mass states ordered? While oscillations can reveal the differences in the mass eigenstates, they don't necessarily tell us about the hierarchy of the mass spectrum - ie, whether $m_{3}>m_{1}$ (normal hierarchy) or $m_{3}<m_{1}$ (inverted hierarchy). Knowledge of the hierarchy of mass states is important in interpreting results from neu- 
trinoless double-beta decay experiments and can provide critical input to astrophysical models of the evolution of the early universe [20].

These questions are motivating a new generation of neutrino experiments at both short and long oscillation baselines. In the next section, we introduce the technology that underlies many these efforts, the liquid argon time projection chamber (LArTPC).

\subsection{LArTPCs for neutrino detection}

Given the extremely small interaction cross-section of neutrinos, a neutrino detector must compensate by offering a large volume of target material in which the neutrinos can interact. Liquid argon (LAr) is a relatively inexpensive noble element, making it well-suited as a target medium for neutrino detection. It turns out it offers several other advantages for neutrino physics as well, which will be addressed later.

Time projection chambers (TPCs) rely on the the collection of free electrons produced along the trails of ionizing particles in a medium (traditionally gaseous) by drifting them across a uniform electric field toward an "anode" of wire planes equipped with readout electronics. Such detectors are capable not only of reconstructing particle tracks in two and three dimensions (2D and $3 \mathrm{D})$, but also act as total-absorption calorimeters since nearly all energy a particle loses goes into producing ionization electrons and scintillation light which can be observed (see Section 3.2). While the use of LAr as a working medium in ionization chambers was first proposed in 1974 by W. J. Willis and V. Radeka [21], it was Carlo Rubbia who applied the idea toward neutrino detection in 1977 [22, 23]. The cartoon in Figure 2.3 illustrates the operation of a LArTPC for detecting the final states from neutrino interactions.

LArTPCs offer several key advantages for neutrino physics. First, LAr is relatively dense compared to gaseous media that were traditionally used in TPCs, resulting in more Ar atoms per unit area and thus increasing the probability of neutrino interactions. The 


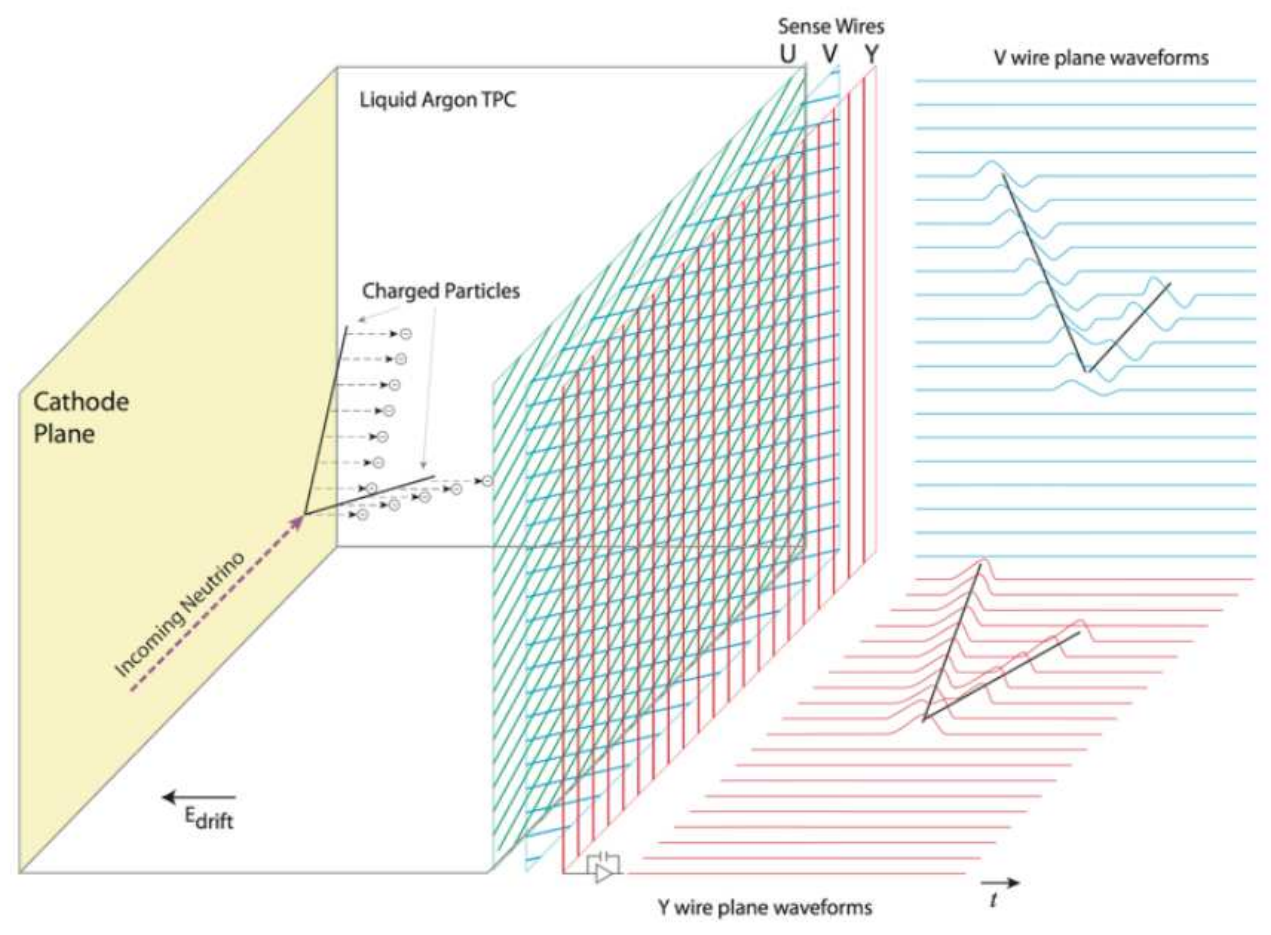

Figure 2.3: Illustration of charged particles emerging from a neutrino interaction and producing ionization tracks in a LArTPC.

increased density also results in a higher rate of energy loss for charged particles, and thus larger and more visible signals on the wires.

LArTPCs are currently being employed to address some of the burning questions in neutrino physics outlined in Section 2.2. The search for sterile neutrino states is being carried out at Fermilab through the Short-Baseline Neutrino (SBN) Program, which employs three LArTPCs - SBND [7], MicroBooNE [24], and ICARUS [23] - along a beam of primarily $\nu_{\mu}$ over a baseline of $\approx 600 \mathrm{~km}$. Results from SBN will address the low-energy excess observed by MiniBooNE and LSND and discover or set new limits on the existence of a sterile neutrino state with $\Delta m^{2} \sim 1 \mathrm{eV}^{2}$. The ability of the LArTPC to spatially reconstruct particle tracks will be a critical factor in this goal since this will allow for the distinction of electronfrom photon-induced electromagnetic (EM) showers. These two topologies produced similar signals in MiniBooNE, a detector which looked for rings produced by Cherenkov light, but LArTPCs are able to exploit two important identifying features. A photon, being a neutral 
particle, will travel some distance from the neutrino interaction point before pair-producing and forming an EM shower, leaving a separation "gap" between the interaction point and the beginning of the shower in which no charge is deposited. However, an electron will begin showering immediately, leaving no such gap. The rate of energy loss $(d E / d x)$ will also be approximately twice as high at the start of photon showers due to ionization from an electron-positron pair produced from the photon. The LArTPCs in SBN will more effectively identify these two topologies, thus minimizing background in an appearance search for $\nu_{e}$.

The Deep Underground Neutrino Experiment (DUNE) [9, 10] represents another prominent example of LArTPC technology at the forefront of neutrino physics. DUNE will consist of a LArTPC built $1.5 \mathrm{~km}$ underground at the Sanford Underground Research Facility (SURF) in South Dakota, USA. This "far detector" will be a modular LArTPC comprised of four separate volumes, each 10 kilotons in volume. DUNE's far detector will detect neutrinos produced 1,300 km away at Fermilab's Long Baseline Neutrino Facility (LBNF). One of the chief goals of DUNE will be to determine whether neutrinos violate CP-symmetry. For example, expanding the terms in the probability distribution in Equation 2.7 results in CP-odd terms, dependent on the CP-violating phase $\delta$, which differ in sign between $\nu$ and $\bar{\nu}$. If $\delta \neq 0$ these result in measurable differences in the oscillations of $\nu$ versus $\bar{\nu}$. Results from DUNE will also be able to determine the neutrino mass hierarchy.

\subsection{Studies enabled by a large underground LArTPC}

The far detector of DUNE, given its location $1.5 \mathrm{~km}$ underground, will be shielded from most cosmic rays that plague LArTPCs at the surface with passing cosmic muons which overlap with the readout window. This will give DUNE a relatively "quiet" environment in which neutrino events will be isolated both spatially and temporally without complications from overlapping cosmic muons. This, together with its massive size (40 kt of active LAr volume) and low energy threshold ( $\gtrsim 1 \mathrm{MeV})$, will expand DUNE's physics reach toward more exotic studies including searches for nucleon decay and detection of neutrinos of as- 


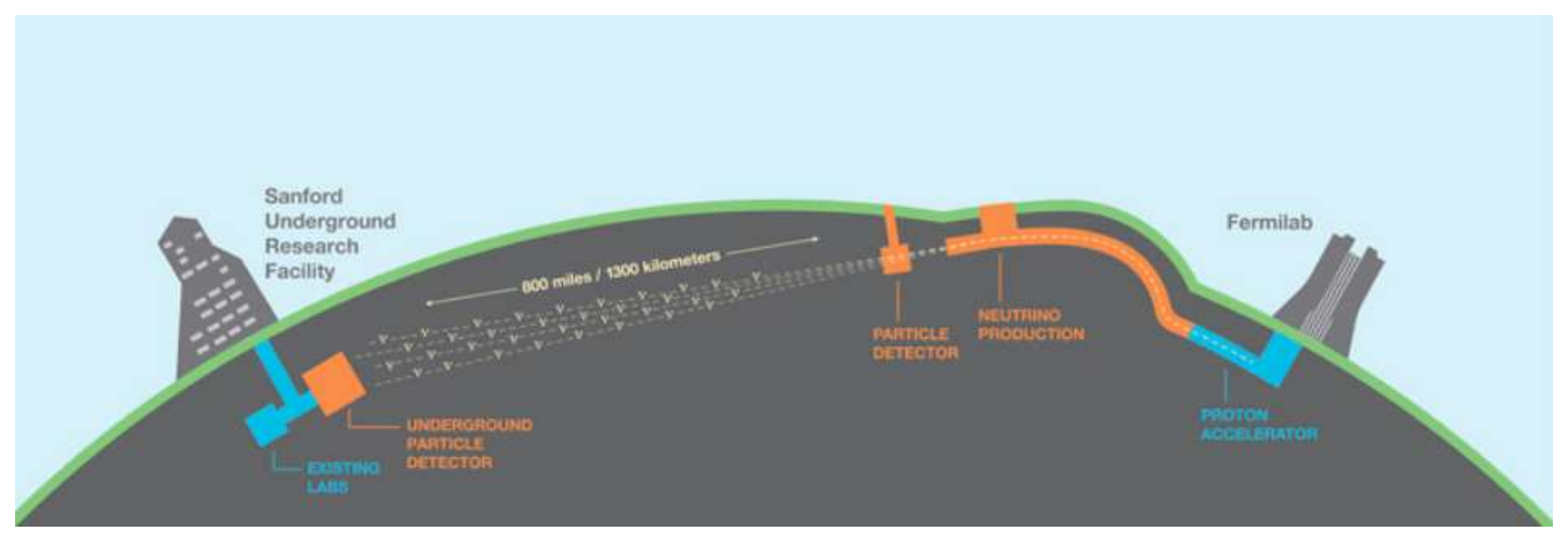

Figure 2.4: The DUNE and LBNF project. A neutrino beam is produced at Fermilab and directed through the earth toward the DUNE far detector located $1.5 \mathrm{~km}$ underground [10].

trophysical origin [9]. Here we describe the physics of core-collapse supernovae neutrinos as well as neutrinos produced in the Sun, and touch upon what DUNE will be able to teach us about particle physics and astrophysics through related studies.

\section{Core-collapse supernova neutrinos}

The iron cores of massive stars, as they age, continuously lose energy which is carried away by neutrinos from pair-annihilation and plasmon decay. This lost energy results in the core continuing to collapse in on itself. Over time, more iron is accreted onto the core from the burning of lighter elements in its surrounding layers. Eventually the core reaches a critical mass (about 1.4 times the mass of the Sun) at which the density and temperature are so high that electrons are absorbed into protons and some of the iron is disintegrated by thermal photons. With the outward pressure suddenly absent, the core collapses in on itself in freefall until nuclear densities are reached ( $\sim 10^{-2}$ seconds), after which it rebounds as an

outward-propagating shock wave forms. At these extreme densities $\left(\rho \sim 10^{12}-10^{14} \mathrm{~g} / \mathrm{cm}^{3}\right)$ even neutrinos cannot propagate freely without significant scattering [9].

The gravitational energy of the collapsed core is, at this point, stored among electrons and $\nu_{e}$ which are unable to immediately escape. When they do escape, about $99 \%$ of the 

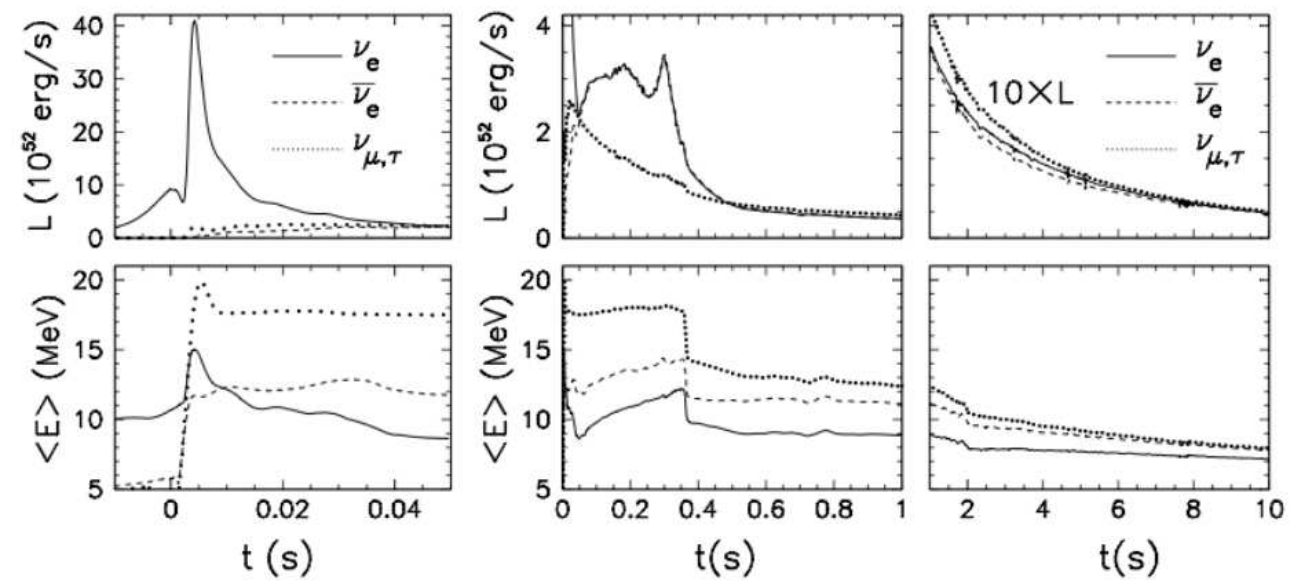

Figure 2.5: Core-collapse supernova $\nu$ signals expected based on the Basel model for a 10.8 solar-mass star. The top row shows the intensity over time, while the bottom row shows the average energy over time, separated by neutrino flavor [25, 26].

core's binding energy is released in a burst of $\nu$ of all flavors - about $10^{58}$ in total, each with an energy of approximately $10 \mathrm{MeV}$. This neutrino emission from the supernova (SN) event persists for about 10 seconds total, including an initial short "neutronization" burst of $\nu_{e}$ at $t<0.01 \mathrm{sec}$, followed by an accretion phase at $t \sim 0.5-1 \mathrm{sec}$ in which a variety of $\nu$ flavors are emitted as stellar matter continues to fall into the core and delays the progress of the outgoing shock-wave. Finally there is a "cooling phase" characterized by a gradual decrease in the $\nu$ intensity that extends for $\sim 10 \sec [9]$. Plots of the neutrino flux over time for core-collapse SN, based on simulations, are shown in Figure 2.5.

A star can also undergo an "electron capture" (EC) type SN in which the final stages of burning are never reached. The $\nu$ signal from an EC SN is similar to that from core-collapse $\mathrm{SN}$ in terms of time evolution and energy spectra.

The last (and only) observation of $\nu$ from a supernova was from SN1987A in 1987. Both the IMB [27] and Kamiokande-II [28] water Cherenkov detectors saw a $\nu$ signal in coincidence with the SN, with a grand total of 19 detected $\nu_{e}$ arriving over a $\sim 10$ second period between the two of them. Thanks to its large active volume, DUNE will (in principle) be able to observe a substantially larger signal! As evidenced in Figure 2.6, LAr is most sensitive to the $\mathrm{CC}$ interaction of electron-type neutrinos, $\nu_{e}+\mathrm{Ar} \rightarrow e^{-}+K^{*}$. For a core-collapse $\mathrm{SN}$ 


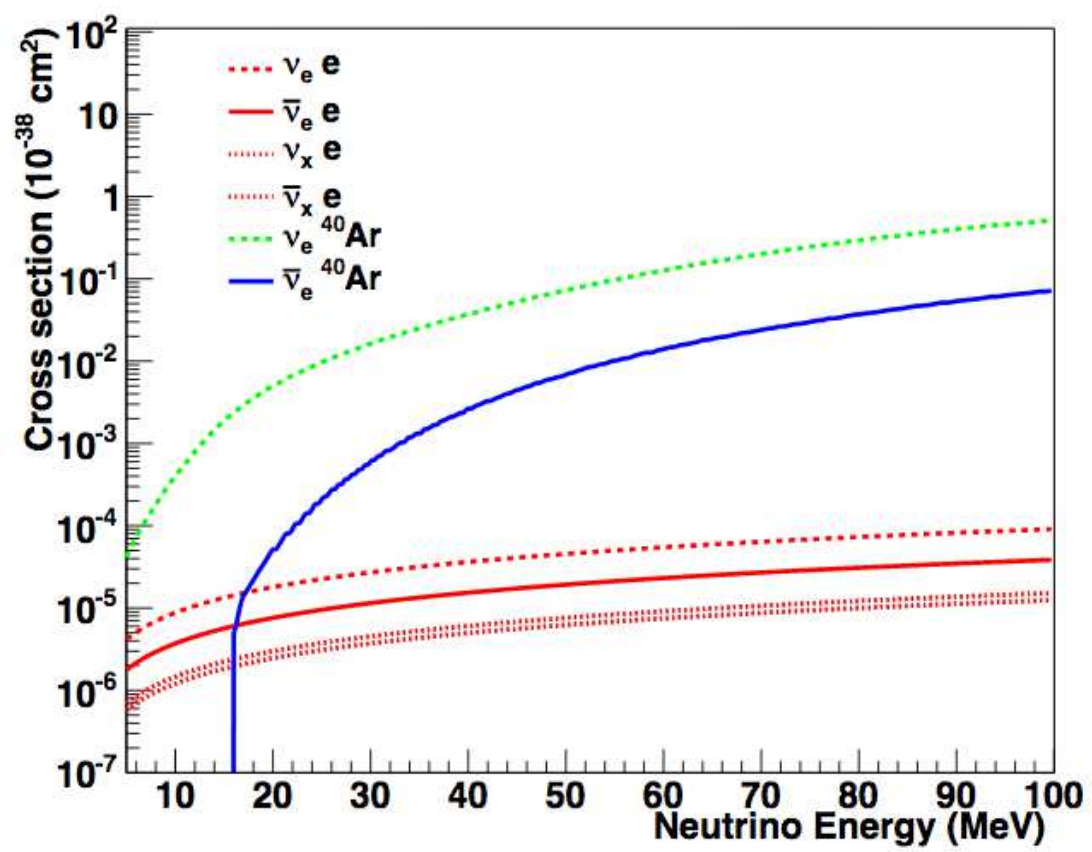

Figure 2.6: Cross-sections for neutrino interactions relevant to supernova emission [29, 30].

10 kiloparsecs (kpc) away, DUNE is expected to see 2700-3300 CC- $\nu_{e}$ interactions total [9]. In Figure 2.7, the number of expected events is plotted versus time (left) and energy (right).

\section{Solar neutrinos}

There are several avenues through which DUNE could make significant contributions to particle physics and astrophysics by studying neutrinos from the Sun. For example, presently there exists a $\sim 2-\sigma$ discrepancy between the best-fit measurement of $\Delta m_{12}^{2}$ from solar neutrino experiments compared to those from reactor-based experiments. While the JUNO [32] experiment will soon improve the constraints on the reactor measurements, additional improvement on the solar measurement is needed in order to fully break the ambiguity and potentially probe new physics. In addition, precise measurements of the flux of neutrinos from ${ }^{8} \mathrm{~B}$ beta-decay $\left(E_{\nu}=18 \mathrm{MeV}\right)$ and the discovery of the hep neutrino flux would help increase our understanding of solar astrophysics [11]. The advantages DUNE offers for solar neutrinos mirror those that enable the detection of SN neutrinos: an inherent overburden 

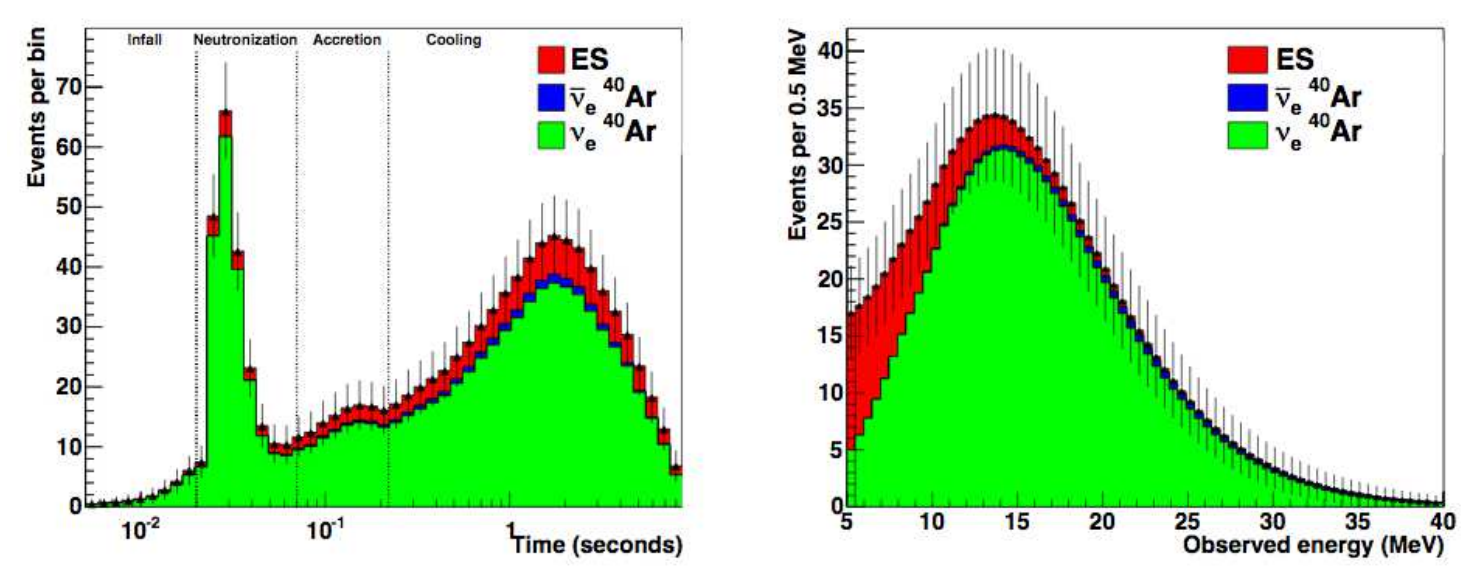

Figure 2.7: Simulated time-dependent $\nu$ signal in $40 \mathrm{kt}$ of LAr from an electron capture SN $10 \mathrm{kpc}$ away (left), and the energy spectrum of $\nu$ integrated over arrival times (right) [31].

due to being located underground as well as a low-energy threshold down to a few $\mathrm{MeV}$. However, it is expected that some modifications to the baseline design plans would need to be implemented in order to fully realize DUNE's potential for solar neutrino studies - specifically, better data acquisition capabilities and improved rejection of $\sim \mathrm{MeV}$ backgrounds from neutrons produced in the rocks surrounding the detector and capturing on Ar within the fiducial volume [1]. Figure 2.8 shows the expected distributions of visible energy from solar neutrino interactions in DUNE.

\subsection{Motivation for low-energy calorimetric capabilities}

As evidenced in Figures 2.7 and 2.8, astrophysical $\nu$ events extend to the lowest energies accessible in DUNE $\left(E_{\nu} \sim 5-50 \mathrm{MeV}\right)$. DUNE's ability to accurately resolve energies down to this level is critical to enabling studies of $\mathrm{SN}$ and solar $\nu$, since poor energy resolution will have the effect of "smearing" low-energy backgrounds to higher energies, crowding out signal regions and degrading the sensitivity of analyses.

For $\mathrm{SN} \nu$, an anticipated energy resolution of $\sigma / E<10 \%$ in the $5-100 \mathrm{MeV}$ range will be needed in order to effectively resolve features in the $\mathrm{SN} \nu$ energy spectrum and probe the relevant physics [12]. For solar $\nu$, the estimated requirement is even more stringent at 

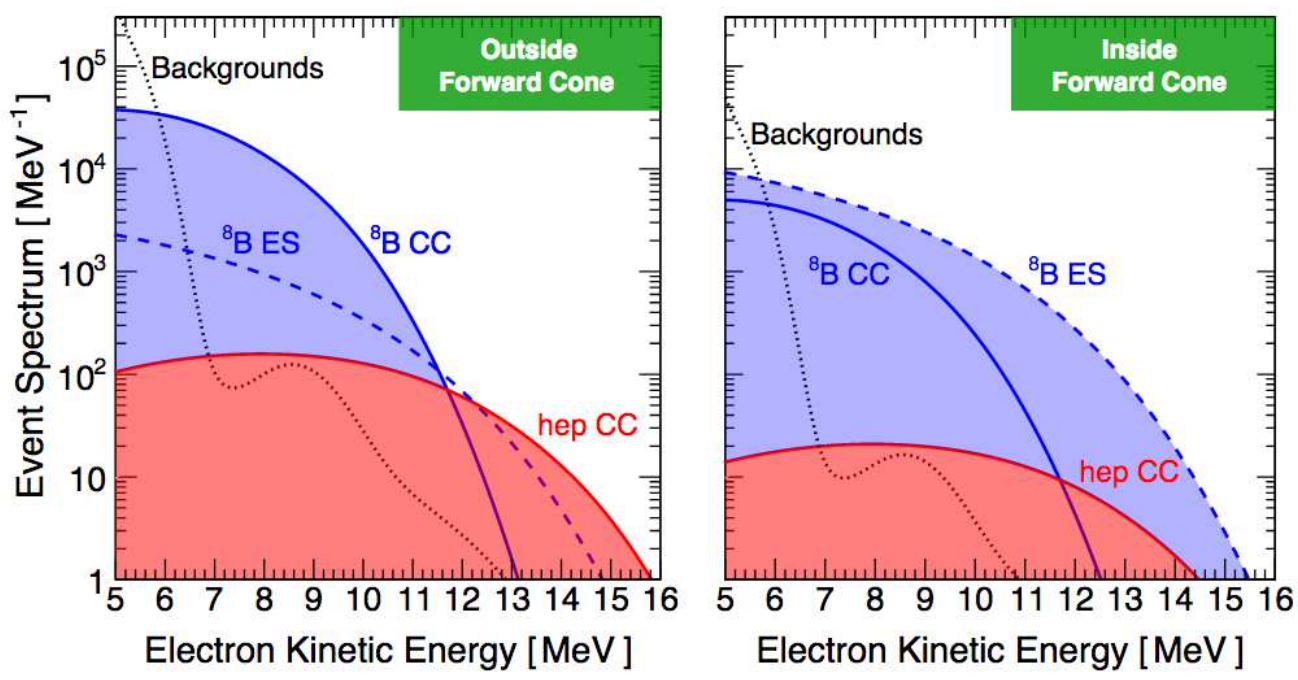

Figure 2.8: Visible energy expected from solar ${ }^{8} \mathrm{~B}$ and hep $\nu$ signal and background in DUNE over a total exposure of $100 \mathrm{kton}$ years for CC-interactions and elastic scattering (ES) interactions. Looking either inside (left) or outside (right) a forward-going cone of half-angle $40^{\circ}$ changes the relative spectra for different event types [11].

$\sigma / E<7 \%$ in order to ensure backgrounds from the end-point energies of products from the ${ }^{42} \mathrm{Ar}$ and ${ }^{222} \mathrm{Rn}$ decay chains $(\approx 3.5 \mathrm{MeV})$ are low enough to avoid appreciably contaminating the low-energy tail of the signal [11].

Good energy reconstruction at low energies will also enhance the reach of oscillation analyses in SBN and DUNE detectors through more efficient tagging of 4.1-MeV muons from pion decay-at-rest and of $<53 \mathrm{MeV}$ Michel electrons from stopping muons. Tagging such features will help identify final-state topologies from $\nu$ interaction events [12].

Several LArTPCs have performed analyses from which energy resolution estimates were extracted for low-energy electrons using decay electrons from stopping cosmic muons. These are commonly referred to as Michel electrons and exhibit an energy distribution ranging from 0-53 MeV, which has significant overlap with the energy range relevant for astrophysical $\nu$ studies outlined earlier (Michel electrons will be discussed in greater detail in Chapter 5).

In a study carried out by the ICARUS experiment [23], the spectrum of energy deposited directly by the decay electron ionization tracks $\left(E^{\text {ion }}\right)$ was reconstructed and compared to $\mathrm{MC}$, and the good data-MC agreement was used to justify the simulation of a sample of 


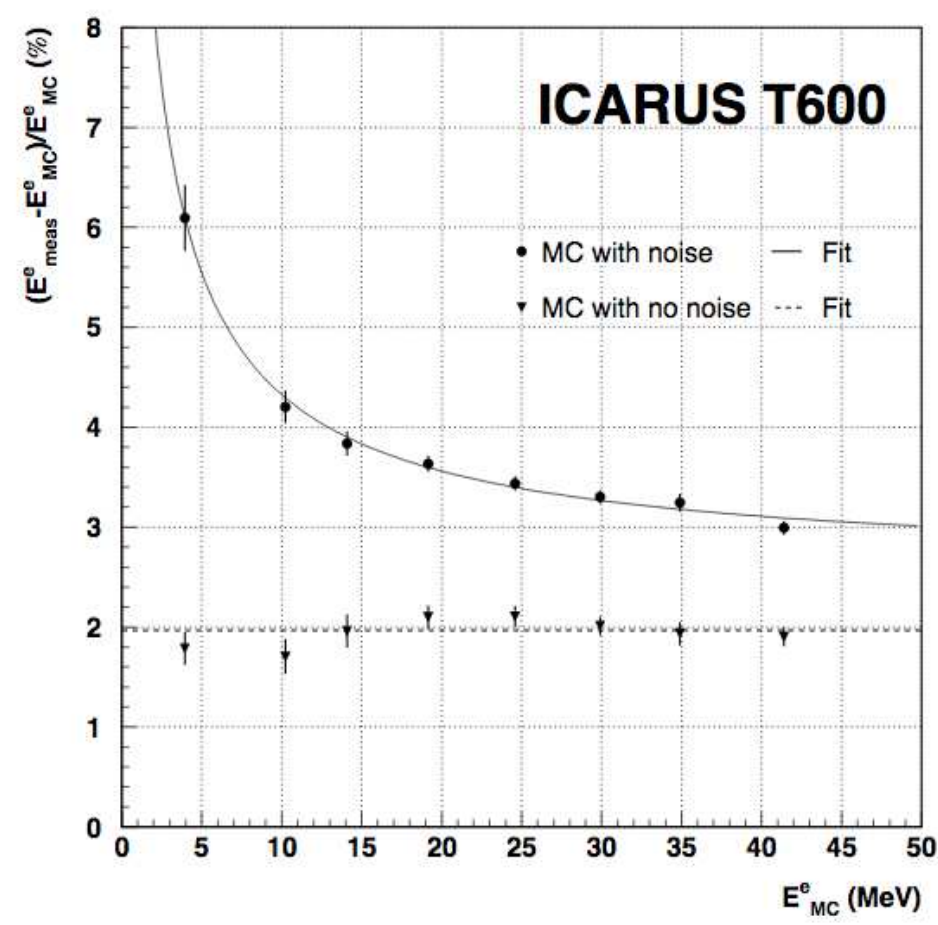

Figure 2.9: Energy resolution in ICARUS for low-energy electron based on MC studies. Only the ionization induced by the electron is considered for both reconstructed and truth information [33].

isolated low-energy electrons to estimate energy resolution. The resulting energy resolution curves from ICARUS are presented in Figure 2.9, taking the resolution from the width of a fitted Gaussian function to the distribution of $\delta E=\left(E_{\text {meas }}^{\text {ion }}-E_{\mathrm{MC}}^{\mathrm{ion}}\right) / E_{\mathrm{MC}}^{\mathrm{ion}}$ at specific values of $E_{M C}^{\text {ion }}$. Fitting these points to a function of the form $f(E)=a / \sqrt{E[\mathrm{MeV}]} \oplus b$, they $a=(11 \pm 1) \%$ and $b=(2.5 \pm 0.3) \%$, with the errors on each value arising from statistics [33]. The first term in this formulation $(a / \sqrt{E})$ models the expected contribution due to electronic noise on signals read out from the wires (from which deposited charge is extracted); the second term $(b)$ represents the contribution due to inherent reconstruction effects related to charge hit-finding, hit-integration, and clustering. This equation is commonly used to characterize the calorimetric resolution in detectors of all kinds.

The MicroBooNE experiment [24] also studied Michel electrons from stopping cosmic muons. Once again, an energy spectrum was reconstructed and compared to MC in order to validate the simulation. The energy resolution was then evaluated from MC, though with 
several key differences relative to the ICARUS study which must be noted. For example, MicroBooNE looked only at the energy of the Michel electron in the muon decay topology (not isolated electrons as was the case in ICARUS's MC study), and they used the original electron's kinetic energy as the "truth" reference energy for calculations of resolution instead of only the deposited energy. They also separately considered the energy constructed from both the direct electron ionization, $E^{\text {ion }}$ (similarly to ICARUS) as well the "total" energy $\left(E_{e}\right)$ by including displaced bremsstrahlung photon-induced charge depositions. They found a fractional energy resolution of $\sigma / E \sim 30 \%$ for the electron ionization alone, which improved to $\sigma / E \sim 20 \%$ after the inclusion of radiative photons. Unfortunately, MicroBooNE was unable to reconstruct a "time-zero" $\left(T_{0}\right)$ for these events which is necessary in order to correct for electron attenuation from impurities during the drift as explained in Section 3.3 . Contributions from cosmic muon contamination further degraded the achieved resolution since muon tracks and delta-rays (knock-off ionization electrons from the muon track) could be clustered together within the electron shower by accident. These two caveats, along with their differences in the definition of energy resolution, make direct comparisons to the ICARUS result difficult.

A recent study performed by the ArgoNeuT experiment [35, 36] measured energies of $\mathrm{MeV}$-scale de-excitation photons from $\mathrm{Ar}$ and deposits from inelastic scattering of neutrons produced in neutrino-Ar interactions [37]. This represents a remarkable milestone for LArTPC calorimetry, extending the accessible energy range down to $\sim 1 \mathrm{MeV}$. Successfully tagging de-excitation gammas may also help in identifying CC- $\nu_{e}$ interactions from SN and solar $\nu$. Since the focus of this thesis is on low-energy electrons, these results from ArgoNeuT will not be discussed in detail.

\subsection{The case for scintillation light}

Absent from our discussion thus far has been the role scintillation light plays in LArTPC reconstruction. Since it propagates several orders of magnitude faster than the drifted elec- 
trons, its detection provides a useful reference point in time $\left(T_{0}\right)$ corresponding to the event. This information is essential for properly measuring the absolute spatial coordinates of charge hits along the drift direction ( $\hat{x}$ as is common convention), which is necessary for estimating and correcting for attenuation of electrons by residual impurities along their drift path. Good timing resolution also aids in background mitigation for accelerator-based oscillation experiments through the requirement that an event be triggered coincidentally with the time structure of the pulsed beam. Timing information may also improve particle identification for delayed decay electrons from stopping muons [38].

Both SN and solar $\nu$ studies can benefit from a powerful light collection capability. For example, if optical flashes can be matched spatially with their associated electron from SN $\nu_{e}$ interactions, the temporal structure of the $\mathrm{SN} \nu$ signal can be measured [12]. Light also offers a way to potentially trigger readout for astrophysical $\nu$ interaction events [11, 12].

In the chapters that follow, an additional use for scintillation light is explored: enhanced calorimetry. First we describe the physics of energy deposition in LAr and how this light is actually produced (alongside the ionization charge). Then, studies carried out in the LArIAT testbeam experiment are presented which demonstrate that the energy resolution for low-energy electrons benefits from the inclusion of scintillation light. 


\section{3}

\section{Charge and Light Production in Liquid Argon}

In this section, the physics of energy deposition by charged particles in LAr is reviewed. Mechanisms of ionization charge and scintillation production are used to motivate calorimetric techniques in which information about both quantities is combined.

\subsection{Energy loss by charged particles in matter}

Charged particles lose energy $(E)$ primarily by ionizing surrounding atoms of the medium within which they traverse. At moderately relativistic energies $(0.1 \lesssim \beta \gamma \lesssim 1000)$ and materials of intermediate atomic number $(Z)$, a particle's stopping power - or average energy loss $(d E)$ per unit length $(d x)$ - is well-described by the Bethe-Bloche equation [39],

$$
\left\langle-\frac{d E}{d x}\right\rangle=K z^{2} \frac{Z}{A} \frac{1}{\beta^{2}}\left[\frac{1}{2} \ln \left(\frac{2 m_{e} c^{2} \beta^{2} \gamma^{2} T_{\max }}{I^{2}}\right)-\beta^{2}-\frac{\delta}{2}\right]
$$

where $z$ is the number of valence electrons of the medium, $A$ is the medium's atomic mass, $\beta$ is the ratio of the particle's velocity to that of light $(v / c), m_{e}$ is the electron mass, $\gamma$ is the Lorentz factor $\left(=1 / \sqrt{1-\beta^{2}}\right), I$ is the mean excitation energy of the atoms of the medium, and $T_{\max }$ is the maximum kinetic energy that a particle of mass $M$ can impart onto a free electron in a single collision:

$$
T_{\max }=\frac{2 m_{e} c^{2} \beta^{2} \gamma^{2}}{1+2 \gamma m_{e} / M+\left(m_{e} / M\right)^{2}}
$$

The term $\delta$ corrects for the density effect which manifests at higher momenta, and $K=$ $4 \pi N_{A} r_{e}^{2} m_{e} c^{2}$ where $N_{A}$ is Avogadro's number and $r_{e}$ is the classical electron radius.

The total energy loss for positive muons in copper is shown in Figure 3.1. Only in the "Bethe-Bloch" region is $d E / d x$ well-modeled by the above relationship; for higher energies, radiative effects begin to dominate in which emitted photons carry away the particle's energy, 


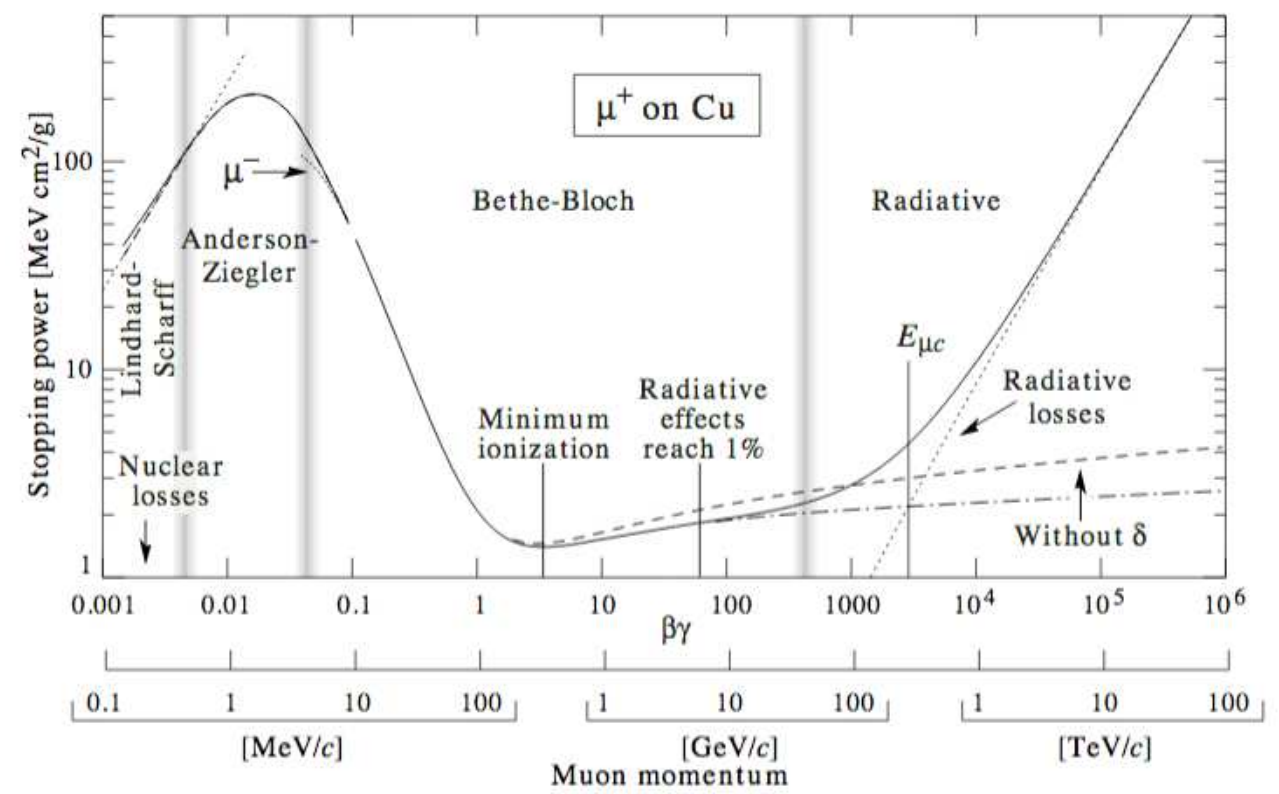

Figure 3.1: Stopping power for muons in copper as a function of energy and momentum 39, 40.

and at lower energies the particle's velocity becomes comparable to those of atomic electrons and Equation 3.1 loses its applicability. The rate of energy loss directly to ionization, as opposed to radiative photons, is often termed the electronic stopping power.

Stopping power is characterized by a broad region of minimal energy loss as seen, for example, in Figure 3.1 at $\beta \gamma \approx 3$ (or a momentum of approximately $500 \mathrm{MeV} / \mathrm{c}$ ). The location of minimal ionization density is dependent on particle mass, typically falling in the range $3.0<\beta \gamma<3.5$. Most particles relevant to high-energy experimental physics - for example, cosmic-ray muons - have energies that place them within this valley of low- $d E / d x$ and are thus termed "minimal ionizing particles" or MIPs.

The electronic energy loss of a moving particle, while on average described by BetheBloch, is subject to stochastic fluctuations. This results in a probability distribution of energy loss per interaction that follows a highly-skewed Landau. Figure 3.2 illustrates this by showing the energy loss distributions for pions traversing a range of different silicon thicknesses. For this reason, the average energy loss $\langle d E / d x\rangle$ tends to be a difficult metric to measure experimentally, and the most probable value (MPV) is preferred. For a particle 


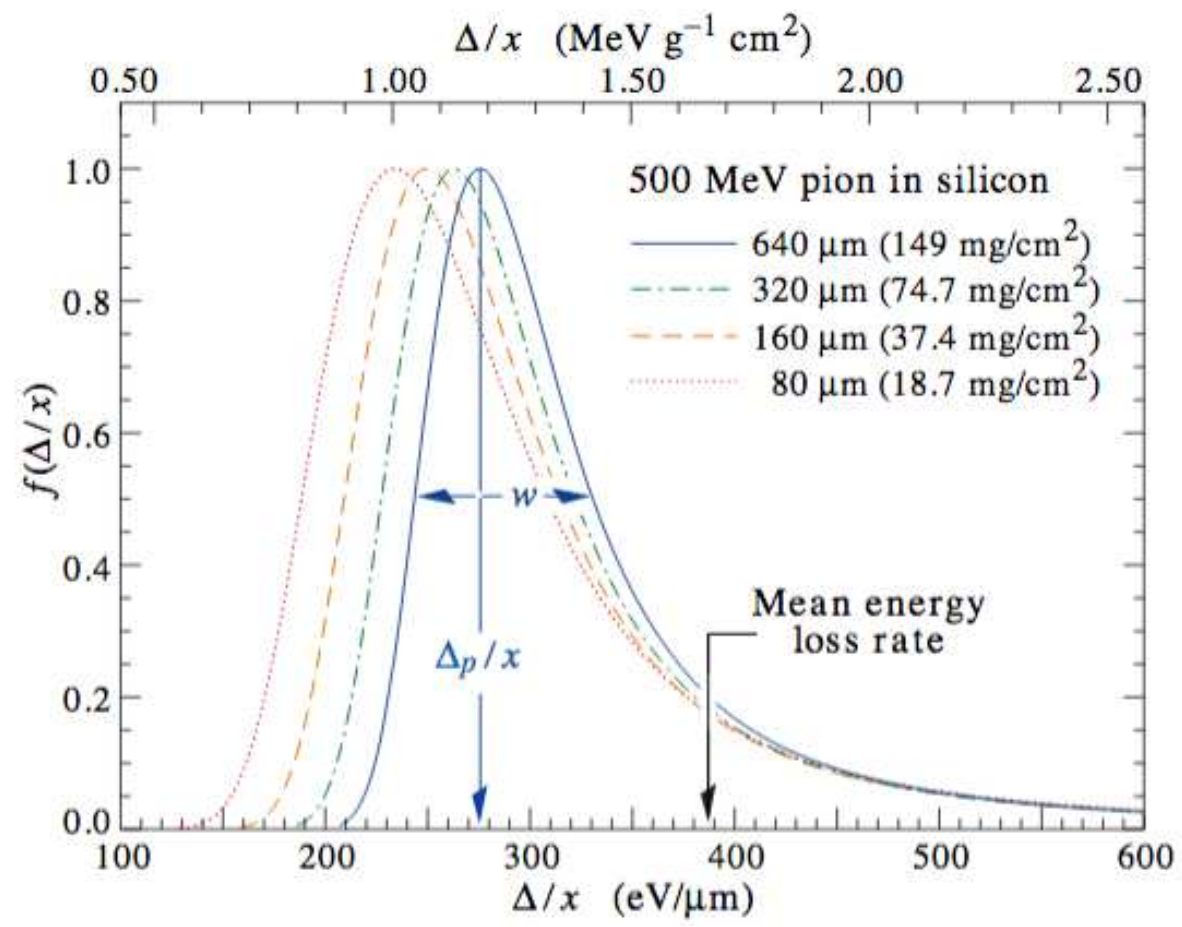

Figure 3.2: Skewed Landau energy loss probability distribution for pions traversing silicon [39].

traversing a material of thickness $x$, the MPV of the total energy loss, $\Delta_{p}$, is described by

$$
\Delta_{p}=\eta\left[\ln \left(\frac{2 m_{e} c^{2} \beta^{2} \gamma^{2}}{I}\right)+\ln \left(\frac{\eta}{I}\right)+j-\beta^{2}-\delta(\beta \gamma)\right]
$$

where $\eta=\frac{1}{2} K(Z / A)\left(x / \beta^{2}\right)$. In Figure 3.3 , both $\langle d E / d x\rangle$ and $\Delta_{p} / x$ are plotted as functions of momentum for several charged particle species traversing a length of $4 \mathrm{~mm}$ of LAr.

Table 3.1: Physical properties of liquid argon (LAr).

\begin{tabular}{|c|l|c|}
\hline Variable & Description & Value \\
\hline$Z$ & Atomic number & 18 \\
\hline$A$ & Atomic mass & $39.948 \mathrm{~g} / \mathrm{mol}$ \\
\hline$I$ & Mean excitation energy (lone atom) & $188 \mathrm{eV} \mathrm{[45]}$ \\
\hline$\rho$ & Density (liquid phase) & $1.396 \mathrm{~g} / \mathrm{cm}^{3}$ \\
\hline$W_{\text {ion }}$ & Average deposited energy per ionized atom (liquid) & $23.6 \pm 0.3 \mathrm{eV}[41]$ \\
\hline$W_{\mathrm{ph}}$ & Mean ionization/excitation energy (liquid) & $19.5 \pm 1.0 \mathrm{eV}[46,[4]$ \\
\hline
\end{tabular}




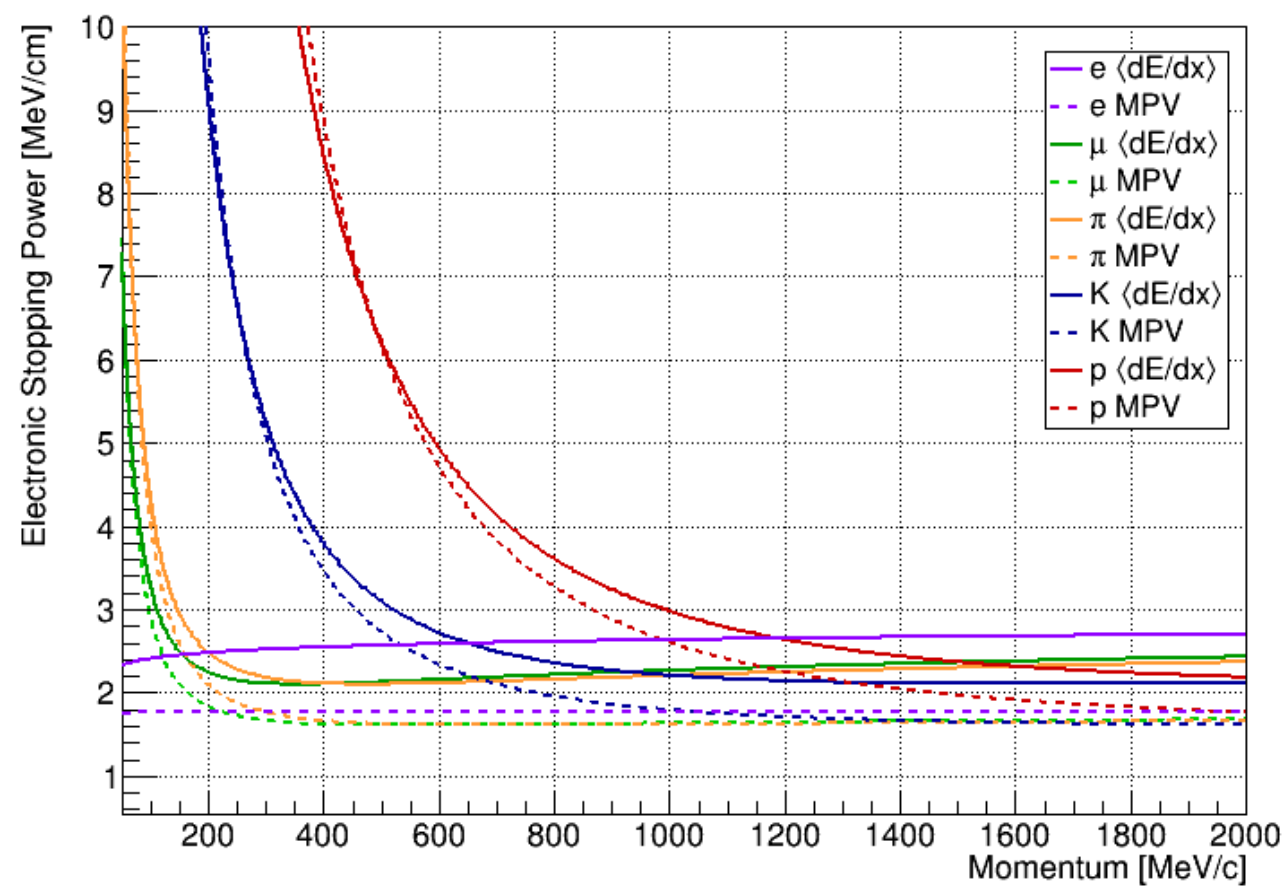

Figure 3.3: The mean electronic stopping power $\langle d E / d x\rangle$ and the most probable electronic stopping power $\Delta_{p} / d x$ (or MPV) for several particle types traversing a 4-mm length of LAr.

\subsection{Ionization, scintillation, and recombination}

The diagram in Figure 3.4 illustrates how energy lost through electronic means by a single particle interaction in LAr is ultimately divided between free electrons and scintillation photons, often referred to here as $Q$ and $L$, respectively. In the following subsections we walk through the physics needed in understanding each step in this chain.

\subsubsection{Ionization and excitation of $\mathrm{Ar}$}

Energy deposited in LAr $(\Delta E)$ is first distributed between some number of excited $\left(N_{\mathrm{ex}}\right)$ and ionized $\left(N_{\mathrm{i}}\right)$ Ar atoms according to an excitation ratio, $\alpha=N_{\mathrm{ex}} / N_{\mathrm{i}}$. The excitation ratio for electrons in LAr is calculated from measurements to be $\alpha=0.21$ [41-43]. This ratio is observed to be higher for more heavily-ionizing particles like alphas.

Through excitation mechanisms and secondary interactions with surrounding bulk Ar atoms, excited argon dimers or excimers, $\mathrm{Ar}_{2}^{*}$, are produced. These short-lived excimers 


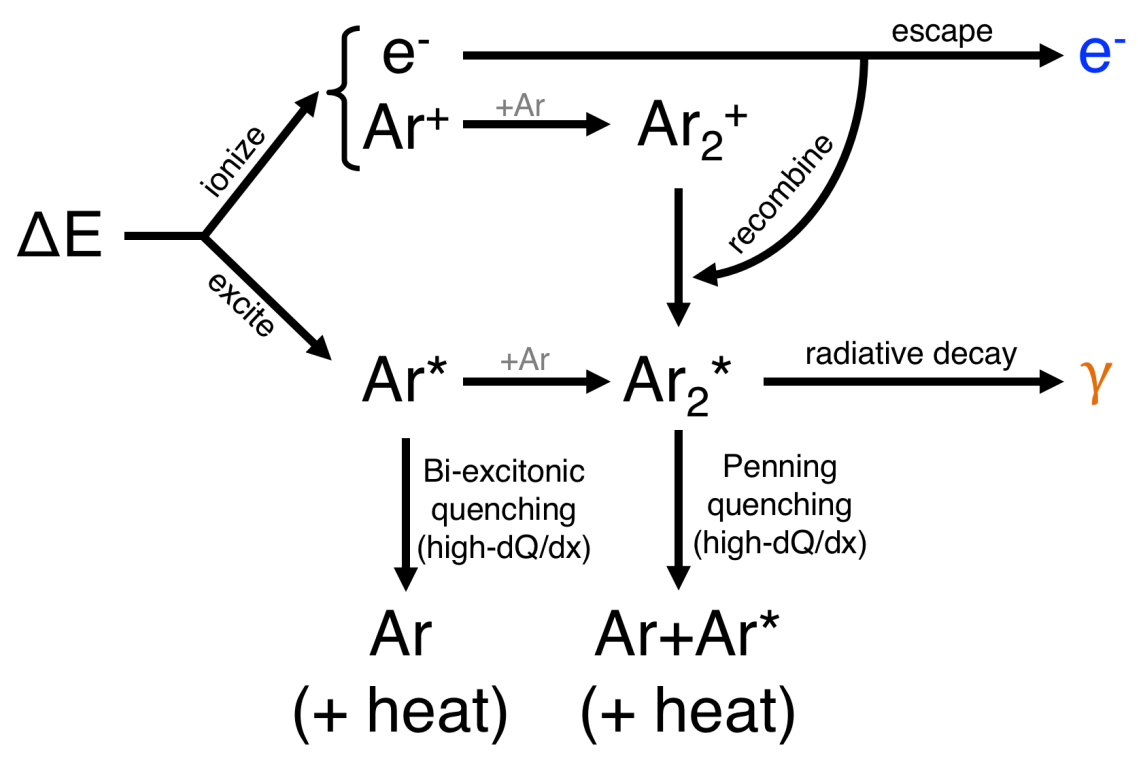

Figure 3.4: Schematic diagram illustrating the production of observable quantities of charge $\left(e^{-}\right)$and scintillation $(\gamma)$ for an energy deposition in liquid argon.

decay to their dissociative ground state by emitting a vacuum ultraviolet (VUV) photon of $\lambda=128 \mathrm{~nm}$ and energy $E=9.69 \mathrm{eV}$ [44]. The excited electron in $\mathrm{Ar}_{2}^{*}$ exists in either the singlet state $\left({ }^{1} \Sigma_{u}^{+}\right)$or the triplet state $\left({ }^{3} \Sigma_{u}^{+}\right)$configuration, with decay lifetimes of $\tau_{s}=6 \pm 2 \mathrm{~ns}$ [48] and $\tau_{t}=1300 \pm 60 \mathrm{~ns}$, respectively [44]. The ratio of singlet to triplet excimers formed, $I_{s} / I_{t}$, is believed to be dependent on the local excitation density along a particle track, enabling particle identification based on the observed ratios of "fast" singlet-decay and "slow" (or "late") triplet-decay components of the light [49]. For electrons, this ratio has most recently been measured as $I_{s} / I_{t}=0.51 \pm 0.05$ [44, 50]. Several ideas have been proposed to explain the dependence of $I_{s} / I_{t}$ on ionization density. One possibility [48] is thermalized free electrons undergoing superelastic collisions with singletstate dimers, changing them to triplet states. This process would then be proportional to the recombination time. Recombination takes place more rapidly for heavily-ionizing particles, leaving less time for this collisional process to take place (resulting in a higher $I_{s} / I_{t}$ ).

The photon-emitting argon excimers $\mathrm{Ar}_{2}^{*}$ are produced through two channels. Schemat- 
ically, the excitation channel proceeds as follows:

$$
\begin{aligned}
\Delta E+\mathrm{Ar} \rightarrow \mathrm{Ar}^{*} & \\
\mathrm{Ar}^{*}+\mathrm{Ar}+\mathrm{Ar} \rightarrow & \mathrm{Ar}_{2}^{*}+\mathrm{Ar} \\
& \mathrm{Ar}_{2}^{*} \longrightarrow 2 \mathrm{Ar}+\gamma
\end{aligned}
$$

In the ionization channel on the other hand, a freed electron $\left(e^{-}\right)$recombines with a molecular argon ion $\left(\mathrm{Ar}_{2}^{+}\right)$to produce a doubly-excited $\mathrm{Ar}^{* *}$ which then undergoes a non-radiative transition producing an exciton, $\mathrm{Ar}^{*}$. This leads to the formation of $\mathrm{Ar}_{2}^{*}$ through interactions with bulk ground-state Ar [47]:

$$
\begin{aligned}
& \Delta E+\mathrm{Ar} \rightarrow \mathrm{Ar}^{+}+e^{-} \\
& \mathrm{Ar}^{+}+\mathrm{Ar} \rightarrow \mathrm{Ar}_{2}^{+} \\
& \mathrm{Ar}_{2}^{+}+e^{-} \rightarrow \mathrm{Ar}^{*}+\mathrm{Ar}+\text { heat } \\
& \mathrm{Ar} *+\mathrm{Ar}+\mathrm{Ar} \rightarrow \quad \mathrm{Ar}_{2}^{*}+\mathrm{Ar} \\
& \mathrm{Ar}_{2}^{*} \longrightarrow 2 \mathrm{Ar}+\gamma
\end{aligned}
$$

\subsubsection{Electron-ion recombination}

The rate of the $e^{-}-\mathrm{Ar}_{2}^{+}$recombination process shown in the ionization channel above is often characterized using the recombination survival probability or recombination factor, $R$, which is the number of escaping electrons $\left(N_{e}\right)$ divided by the original number of electron-ion pairs produced. The recombination factor is dependent in part on the external electric field strength $(\mathscr{E})$. For stronger electric fields, more liberated electrons are drifted away from the deposition region before they can recombine, contributing to the total deposited charge referred to as $Q$. On the other hand, the recombination probability decreases at lower $\mathscr{E}$, resulting in less charge and more scintillation light $L$. The ionization density also plays a 
role in this process since a smaller average separation distance between electrons and ions in the ionization cloud results in more efficient recombination.

Following from the physical intuition outlined above, functional forms predicting the rate of recombination have been derived phenomenologically. The Onsager germinate theory of recombination [51] assumes that a freed electron recombines with its parent ion under the influence of the mutual Coulomb attraction between them. Alternatively, the columnar model of recombination proposed by Jaffe [52] assumes electrons instead reattach primarily to other argon ions nearby. The Jaffe model thus predicts the local density of ionization will be positively correlated with the rate of recombination. Simulations [53] of electron thermalization in liquid argon estimate the probability of germinate recombination at $\approx 10^{-3}$ for typical charge deposition densities. Therefore, the Jaffe model is more relevant in understanding recombination in liquid argon since electrons are far more likely to recombine with other ions in their vicinity. Jaffe theory assumes electrons and ions take on a Gaussian spatial distribution around the particle trajectory, and that this distribution (as well as the charge mobility factors, $\mu$ ) are the same for both. Following these assumptions, the fraction of electrons and ions that survive the recombination process is given by an equation which is now referred to as Birks law [54]:

$$
R_{J}=\left[1+\frac{\alpha N_{0}}{8 \pi D} \sqrt{\frac{\pi}{z^{\prime}}} S\left(z^{\prime}\right)\right]^{-1} \text { where } z^{\prime}=\left(\frac{b \mu \mathscr{E} \sin \phi}{\sqrt{2} D}\right)^{2}
$$

where $\alpha$ is the coefficient of recombination, $N_{0}$ is the number of ion-electron pairs per unit length, $D$ is the diffusion coefficient, $\mathscr{E}$ is the external electric field, and $b=r_{0} \sqrt{4 / \pi}$ where $r_{0}$ is the average ion-electron separation. The angle of the particle's trajectory relative to the electric field is given by $\phi$. The function $S\left(z^{\prime}\right)$, which accounts for the time-dependent overlap of electron and ion distributions, is given by

$$
S\left(z^{\prime}\right)=\frac{1}{\sqrt{\pi}} \int_{0}^{\infty} \frac{e^{-s}}{\sqrt{s\left(1+s / z^{\prime}\right)}} d s
$$


For typical particle trajectories ${ }^{1}$ in LArTPCs, $S\left(z^{\prime}\right) \sim 1$, allowing Equation 3.4 to be rewritten in a simpler form:

$$
R_{J}=\left[1+k_{c} \frac{(d E / d x)}{\mathscr{E} \sin \phi}\right]^{-1}
$$

where all the prefactors have been absorbed into a liquid argon-specific constant, $k_{c}$.

Further simplifications to this model are made by Thomas and Imel [57]. They ignore transverse electron diffusion, which is negligible (on the order of millimeters per meter of drift). They also neglect the mobility of positive ions since the ion drift velocity is at least several orders of magnitude smaller than the electron drift velocity. By dropping these terms and imposing "Box model" boundary conditions on the Jaffe equation, they find

$$
R_{\mathrm{Box}}=\frac{1}{\xi} \ln (\alpha+\xi), \quad \xi=\frac{\alpha N_{0}}{4 a^{2} \mu \mathscr{E}}
$$

The variable $\xi$ incorporates the charge density in a box of size $a$ and volume $V=4 a^{2}$. One can recast $\xi$ in terms of linear charge density,

$$
\xi=\beta(d E / d x)
$$

where $\beta$ is a parameter that would be extracted from fits to data.

Several LArTPC experiments used data to parameterize the recombination survival probability. The ICARUS Experiment [23] used a sample of minimally-ionizing stopping cosmic muons to determine a parameterization of the Birks functional dependence on stopping power

1. The ArgoNeuT analysis used proton and deuterons over an angular range of $\phi=20^{\circ}-80^{\circ}$ in an electric field of strength $481 \mathrm{~V} / \mathrm{cm}$. They estimated the diffusion and mobility of electrons based on electron thermalization, $D / \mu \approx E_{\text {thermal }} \approx 0.01 \mathrm{eV}$, and used $r_{0}=2500 \mathrm{~nm}$. For this range of conditions, $S\left(z^{\prime}\right)$ was found to vary by less than $1 \%$. 558. 
$d E / d x$ in LAr [55]:

$$
\begin{aligned}
R(d E / d x) & =\frac{A}{1+k /(\mathscr{E} \times d E / d x \times \rho)} \\
A & =0.800 \pm 0.003 \\
k & =0.0486 \pm 0.0006(\mathrm{kV} / \mathrm{cm})\left(\mathrm{g} / \mathrm{cm}^{2}\right) / \mathrm{MeV}
\end{aligned}
$$

It is interesting to note that the ICARUS parameterization of Birks Law does not tend toward $R \rightarrow 0$ with $\mathscr{E} \rightarrow 0$ as one would expect based on physical arguments. This implies that even at zero-field, some fraction of freed electrons are expected to escape the recombination process as they diffuse outward and into regions of considerably lower charge density. This idea is supported by experimental results [56].

The ArgoNeuT Experiment [35] parameterized recombination using samples of more heavily-ionizing particles, protons and deuterons. ArgoNeuT also engineered a modified form of the Box model from Equation 3.7 which avoided mathematical pitfalls that manifest for spuriously-high values of $d E / d x$ [58]. This "Modified Box model" recombination is represented as

$$
\begin{aligned}
R(d E / d x) & =\frac{\ln \left[\alpha+\beta^{\prime}(d E / d x) /(\rho \mathscr{E})\right]}{\beta^{\prime}(d E / d x) /(\rho \mathscr{E})} \\
\alpha & =0.93 \pm 0.02 \\
\beta^{\prime} & =0.212 \pm 0.002(\mathrm{kV} / \mathrm{cm})\left(\mathrm{g} / \mathrm{cm}^{2}\right) / \mathrm{MeV}
\end{aligned}
$$

Both the Birks Model (Equation 3.9) and Modified Box Model (Equation 3.12 are plotted as functions of $d E / d x$ for LArIAT's electric field value of $\mathscr{E}=484 \mathrm{~V} / \mathrm{cm}$ in Figure 3.5.

\subsubsection{Production of charge and light}

For heavily-ionizing nuclear recoils, various effects [59 62] are believed to lead to energy also being dissipated into atomic motion (heat) through the non-radiative destruction of 


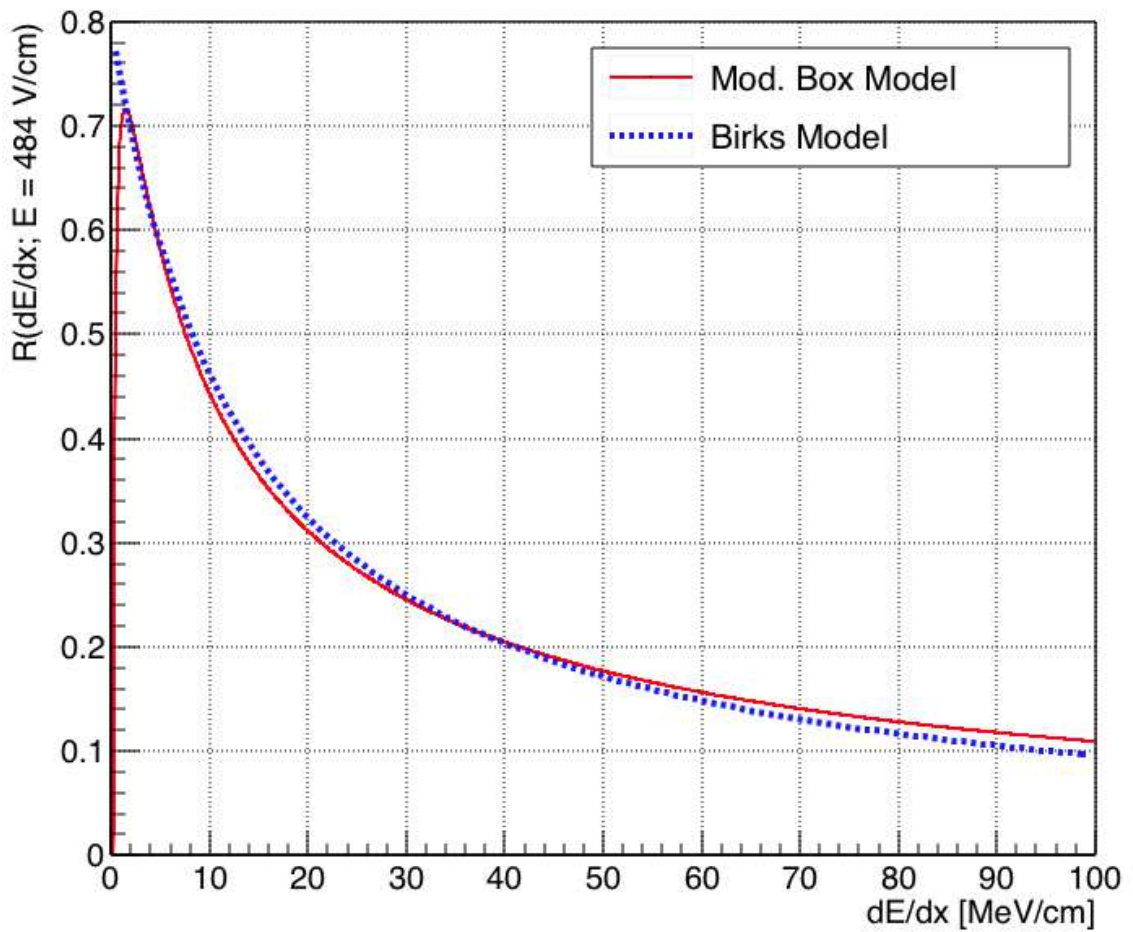

Figure 3.5: Recombination in LArIAT according to the Modified Box Model (using ArgoNeuT parameterization [58]) and the Birks Model (using ICARUS parameterization [55]). The downturn in the Modified Box curve at low- $d E / d x$ is a mathematical artifact of the equation and does not describe actual recombination physics. 
some fraction of $\mathrm{Ar}_{2}^{*}$ as described by Lindhard's theory, bi-excitonic quenching of excitons, and Penning quenching of excimers. For MeV-scale electrons however, we may neglect these effects and assume all the deposited energy goes into observable charge and light and represent them mathematically as

$$
\begin{aligned}
& Q=N_{e}=N_{\mathrm{i}} R \\
& L=N_{\gamma}=N_{\mathrm{ex}}+N_{\mathrm{i}}(1-R),
\end{aligned}
$$

Charge and light are therefore seen to be anticorrelated for electrons, with their sum directly proportional to the total energy deposited,

$$
Q+L=N_{\mathrm{ex}}+N_{\mathrm{i}}=\frac{\Delta E}{W_{\mathrm{ph}}}
$$

where $W_{\mathrm{ph}}=19.5 \pm 1.0 \mathrm{eV}$ [46, 47] is the average energy required to produce an ion or exciton. The quantity $W_{\text {ph }}$ relates to the commonly used ionization work function, $W_{\text {ion }}=$ $23.6 \pm 0.3 \mathrm{eV}$ [41], through the excitation ratio, $W_{\mathrm{ion}}=(1+\alpha) W_{\mathrm{ph}}$.

\subsection{Quenching effects from impurities in LArTPCs}

The processes described so far govern the direct production of ionization electrons and photons at the site of the particle interaction. Here we describe how impurities in the argon can further reduce these quantities prior to their collection in a LArTPC, a process known as quenching. Such impurities $\left(\mathrm{O}_{2}, \mathrm{~N}_{2}, \mathrm{H}_{2} \mathrm{O}\right.$, and $\left.\mathrm{CO}+\mathrm{CO}_{2}\right)$ are commonly found in trace amounts in commercially-available LAr [63]. While multi-stage filtering processes using oxygen reactants and molecular sieves are fairly effective at removing $\mathrm{O}_{2}, \mathrm{H}_{2} \mathrm{O}$ and $\mathrm{CO}+\mathrm{CO}_{2}$ in LAr (down to < 100 parts-per-trillion, or ppt), a small residual fraction of these contaminants can remain, especially if the filter malfunctions or there is a leak or outgassing into the purified LAr volume in which the TPC resides. 


\subsubsection{Charge attenuation during drift}

In a LArTPC, electrons freed by ionization which do not recombine with $\mathrm{Ar}_{2}^{+}$will be directed along the electric field toward the readout wires. However, electrons will attach to electronegative contaminants, especially oxygen, along the way $\left(e^{-}+\mathrm{O}_{2} \rightarrow \mathrm{O}_{2}^{-}\right)$. If it is assumed that the concentration of electrons is much smaller than that of the oxygen $\left(C_{e} \ll C_{O 2}\right)$, and that the concentration of contaminants is uniform throughout the volume, then this attachment process will cause the free electron concentration to decrease over time based on a first-order rate law, with the rate of decrease proportional to the electron concentration:

$$
\frac{d}{d t}\left[C_{e}\right]=-k_{e} C_{O 2} C_{e} \quad \longrightarrow \quad C_{e}(t)=C_{e}^{0} e^{-t / \tau_{e}}
$$

where $C_{e}^{0}$ is the electron concentration at some reference time $t=0, \tau_{e}$ is the electron lifetime and $k_{e}=1 / \tau_{e}$ is the corresponding rate constant [63]. The value of $k_{e}$ depends on the electric

field strength with $k_{e}=5.5 \times 10^{10} \mathrm{lt} \mathrm{mol}^{-1} \mathrm{~s}^{-1}$ [64], though typically the value of $\tau_{e}$ will be measured directly from data from the observed attenuation of charge deposition density $(d Q / d x)$ over drift distance. The calibration method to extract $\tau_{e}$ from data is described in Chapter 6.

\subsubsection{Light quenching}

Non-radiative destruction of photon-producing Ar excimers, $\mathrm{Ar}_{2}^{*}$, can occur through twobody collisions with all kinds of residual impurities. The impurity contributing most prominently to this process is $\mathrm{N}_{2}\left(\mathrm{Ar}_{2}^{*}+N_{2} \rightarrow 2 \mathrm{Ar}+N_{2}\right)$. Similarly as before with charge attenuation, the rate of change in excimer concentration $\left(C_{*}\right)$ is thus proportional to the concentration of contaminants $\left(C_{N 2}\right)$. However, the excimer concentration now has its own time dependence due to the singlet- and triplet-state de-excitations:

$$
C_{*}(t)=C_{t}+C_{t}=C_{s}^{0} e^{-t / \tau_{s}}+C_{t}^{0} e^{-t / \tau_{t}}
$$




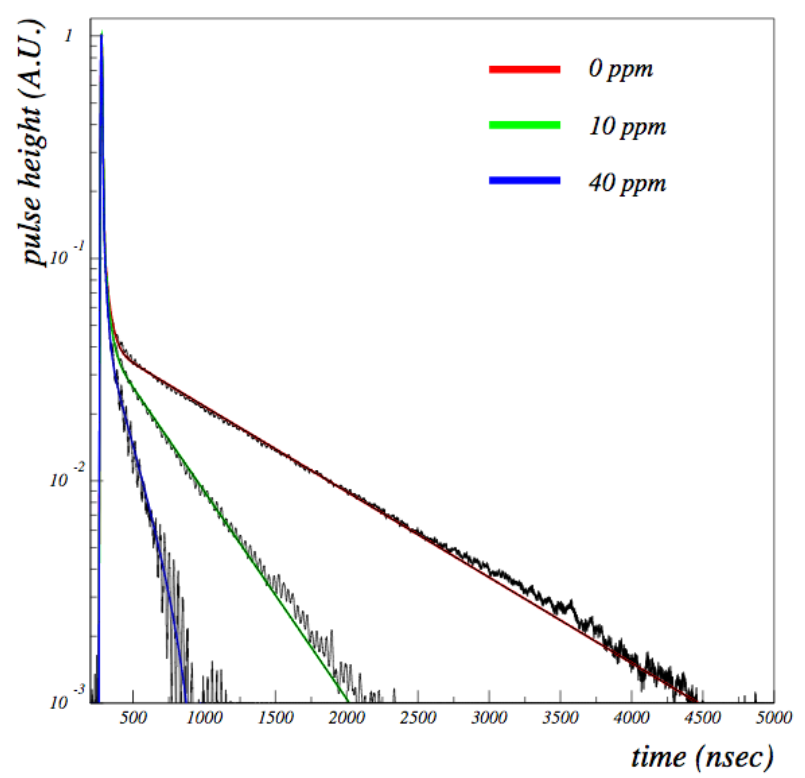

Figure 3.6: Average optical signals for different levels of $\mathrm{N}_{2}$ contamination [65].

where $C_{S}$ and $C_{T}$ are the singlet- and triplet-state excimer concentrations, respectively. The impurity quenching process therefore competes with the radiative de-excitation that produces light $\left(\mathrm{Ar}_{2}^{*} \rightarrow 2 \mathrm{Ar}+\gamma\right)$. Following the same procedure as with the charge attenuation, we arrive at modified time-dependent functions for $C_{S}$ and $C_{T}$ in the presence of LAr impurities:

$$
\begin{aligned}
& C_{s}(t)=C_{s}^{0} e^{-t\left(\tau_{s}^{-1}+\tau_{q}^{-1}\right)} \\
& C_{t}(t)=C_{t}^{0} e^{-t\left(\tau_{t}^{-1}+\tau_{q}^{-1}\right)}
\end{aligned}
$$

where $\tau_{q}$ is the characteristic quenching lifetime (analogous to the electron lifetime $\tau_{e}$ from before). We see that excimer population $j$ now has an "effective" quenched lifetime [63, 65],

$$
\tau_{j}^{\prime}=\left(\frac{1}{\tau_{j}}+\frac{1}{\tau_{q}}\right)^{-1}
$$

The net result of this scintillation quenching is a reduction in the observed lifetimes of the prompt (singlet) and late (triplet) components of the light as well as an overall reduction of factor $\tau^{\prime} / \tau$ in the amount of light that gets produced by each component [65]. In typical 
experimental conditions where LAr has undergone proper filtering, $\tau_{q} \gg \tau_{s}$ and very minimal change is seen in the prompt singlet component $\left(\tau_{s}^{\prime} \approx \tau_{s}=6 \mathrm{~ns}\right)$. However, the triplet component tends to be more vulnerable to this quenching due to its much longer decay time of $\tau_{s}=1.3 \mu \mathrm{s}$. This is demonstrated in Figure 3.6 where average scintillation waveforms are shown for varying levels of $\mathrm{N}_{2}$ contamination in LAr [65], with late-light lifetime dramatically decreasing as contamination levels rise from $0 \mathrm{ppm}$ to $40 \mathrm{ppm}$.

Correcting for light quenching in data thus relies on the knowledge of $\tau_{q}$, which can be estimated based on exponential fits to average optical waveforms. A linear relationship between $\tau_{q}$ and the late-light lifetime measured in averaged optical waveforms, derived from a Monte Carlo study, is presented in Appendix A.

\subsection{Implications for energy reconstruction}

Before $Q$ or $L$ can be used in energy reconstruction, the quenching described previously must be corrected. For $Q$, the correction is straight-forward: each individual charge deposit $q$ is scaled up based on its measured drift time and the known electron lifetime, $q \times e^{t / \tau_{e}}$. For $L$, the correction is not as simple due to the differing levels of quenching in the prompt and late light. These quenching corrections will be presented in Chapter 8 .

Light presents additional complications as well. First, determining the amount of fastand slow-component light is not straightforward since the time distribution of detected light is additionally convoluted with physical propagation time and the re-emission fluorescence from the wavelength shifter tetraphenyl-butadiene (TPB), which has components with lifetimes ranging from $\approx 5$ ns to $3.5 \mu \mathrm{s}$ [67]. Secondly, unlike the electrons which drift directly to the wires at constant velocity, VUV photons emanate isotropically. Typically only a very small fraction of photons are ever detected. Therefore, one must know the fraction of detected photons, $f_{\text {vis }}$, which requires knowledge of the photon visibility throughout the detector (from Monte Carlo) as well as a sufficient number of reconstructed 3D space points. From

there, it is possible to reconstruct the light produced in the event, $L=S \times f_{\text {vis }}^{-1}$, where $S$ is 
the (quenching-corrected) amount of detected light.

For track-like topologies that can be spatially reconstructed, the linear density of energy deposition, $d E / d x$, can be calculated for points along the track and summed to determine the total energy deposited by the particle. However, for more complicated topologies like EM showers which may include many short charge depositions that can't be fully tracked in reconstruction, the $d E / d x$ cannot be determined for all depositions, and an average recombination $\langle R\rangle$ must be assumed:

$$
E=\left(Q \times\langle R\rangle^{-1}\right) \times W_{\text {ion }}
$$

Combining Eqs. 3.15) and (3.17), the deposited energy can be more holistically determined by exploiting the complementarity of $Q$ and $L$ for EM showers, foregoing the need to correct for an assumed recombination:

$$
E=\left(N_{\mathrm{ex}}+N_{\mathrm{i}}\right) \times W_{\mathrm{ph}}=(Q+L) \times W_{\mathrm{ph}}
$$

This recombination-agnostic method is particularly useful for shower-like topologies which, due to the stochasticity of bremsstrahlung radiation and normal Poisson fluctuations in energy deposition, are likely to include charge deposited over a wide variety of $d E / d x$.

The physics of energy dissipation in LAr presented here has motivated the use of scintillation light in energy reconstruction. We now intend to use low-energy electrons in LArTPC data to demonstrate Equation 3.24 in action! This sample will be provided by the LArIAT testbeam experiment, which is introduced in the next chapter. 


\section{4}

\section{The LArIAT Testbeam Experiment}

The LArIAT (Liquid Argon In A Testbeam) experiment [68, 69] ran at Fermilab's Test Beam Facility [70] from 2015-2017. Its primary purpose was for research and development (R\&D) studies related to calibration of the LArTPC technology more generally, though it is also delivering several physics results relevant to the field of neutrino research using liquid argon detectors.

LArIAT's beamline was assembled and commissioned in 2014. Several data-taking campaigns were undertaken throughout LArIAT's lifetime, with the first, termed Run I, beginning in April 2015 and ending in July prior to Fermilab's summer beam shutdown. Run II began in February 2016 and lasted until July. Several important upgrades were made to the TPC readout electronics prior to Run II which resulted in better signal-to-noise $(\mathrm{S} / \mathrm{N})$ on the wires. Modifications were also made to the biasing of the light collection system. These two changes resulted in improved data quality compared to Run I. LArIAT's second major run period was bifurcated into two sub-periods based on the configuration of the light collection system. In Run IIA, only one of the two PMTs was operational; a repair was made prior to Run IIB, after which both PMTs were used. This is described in more detail later. For Run III (2017), wire planes with different wire spacings were tested. However, the data presented in this thesis portends only to Runs I-II, so Run III will not be discussed in detail.

We now describe the detector subsystems relevant to this analysis as well as the Michel electron hardware trigger using scintillation light from stopping cosmic muons.

\subsection{Motivation for LArIAT: physics and R\&D goals}

With interest in liquid argon surging among neutrino physicists, and several LArTPC-based neutrino experiments planned or already running, a dedicated calibration of this emerging technology is invaluable. LArIAT fills this niche through studies of particle response calibra- 
tion, technical R\&D, and several physics topics relevant to current and future liquid argon detectors [69]. Such efforts will provide input to Fermilab's LAr-based neutrino program.

The primary research goals of LArIAT are outlined below:

- Electron/photon shower separation. Charged-current (CC) $\nu_{e}$ interactions are identified by the appearance of an electron EM shower emanating directly from an interaction vertex. Neutral-current (NC) events can produce neutral pions $\left(\pi^{0}\right)$ which decay to two photons, which will (invisibly) travel some distance before pair-producing to form an EM shower. Distinguishing these two events is critical to the success of the SBN Program at Fermilab which is searching for $\nu_{e}$ appearance in a $\nu_{\mu}$ beam to address the low-energy $\nu_{e}$ excess observed in LSND and MiniBooNE. LArTPCs are able to accomplish this thanks to their calorimetric nature and fine spatial granularity. Photon showers are tagged by identifying two key features: (1) a spatial displacement between the interaction vertex and the start of the EM shower; and (2) a linear energy deposition density $d E / d x$ corresponding to two minimally-ionizing particles (MIPs) at the start of the shower. ArgoNeuT has demonstrated this $d E / d x$ separation on a small sample of electrons and photons from $\nu_{e}$ data events [36]. LArIAT will be able to carry out a systematic measure of the efficiency of this method using a large sample of electron showers produced in its tertiary beamline, as well as a sample of photons through $\pi^{0}$ decays and bremsstrahlung radiation from electrons.

- Pion-argon interactions. A major source of systematic uncertainty in neutrino interactions is related to the largely-unknown dynamics of pions interacting with constituents of the Ar nucleus. LArIAT will measure various $\pi$-Ar interaction cross section for the first time, helping to validate nuclear models implemented in simulations. As part of this effort, LArIAT will also facilitate the development of algorithms for identifying the various pion interaction modes on Ar.

- Kaon-argon interactions. The efficient identification of charged kaons $\left(K^{ \pm}\right)$is im- 
portant for proton decay searches which will look for the decay channel $p \rightarrow \bar{\nu} K^{+}$, predicted by various supersymmetry (SUSY) and grand unified theories (GUTs). LArIAT will use the small fraction of kaons present in its beamline to measure $K^{ \pm}$-Ar cross sections and evaluate their reconstruction and identification efficiency.

- Proton and antiproton interactions in LAr. LArIAT will collect a large sample of protons $(p)$ from its positively-tuned tertiary beamline, and a much smaller sample of antiprotons $(\bar{p})$ when the beam is tuned to select negatively-charged particles. A measurement of the $p$-Ar cross section will be possible, as well as the observation of $\bar{p}$-Ar annihilation, the results from which will help in constraining simulated nuclear models which will be used in searches for neutron-antineutron oscillation in DUNE.

- Scintillation light. As discussed in Chapter 3, much of the energy deposited by particles in LAr goes into generating scintillation photons. LArIAT's powerful light collection system, to be described momentarily, allows for studies in which this light is used to enhance calorimetry or in particle identification techniques (similar to those used in dark matter searches) in which the prompt light ratio is used to separate particles based on their $d E / d x$ [49].

\subsection{Beamline}

The charged particles which LArIAT observes originate from the Fermilab Main Injector (MI). Protons are accelerated to $120 \mathrm{GeV}$ in the MI and then directed toward the Fermilab Test Beam Facility (FTBF) [70] where they impinge upon a target to produce a "secondary beam" composed mostly of high-energy pions and protons. The energy of this secondary beam can be tuned to anywhere between $8 \mathrm{GeV}$ and $80 \mathrm{GeV}$.

The secondary beam is used to create what is referred to as LArIAT's "tertiary beam" in the Meson Center enclosure at FTBF. Since the analysis presented in this thesis does not use any beamline information, we will only discuss the components of the tertiary beam in 


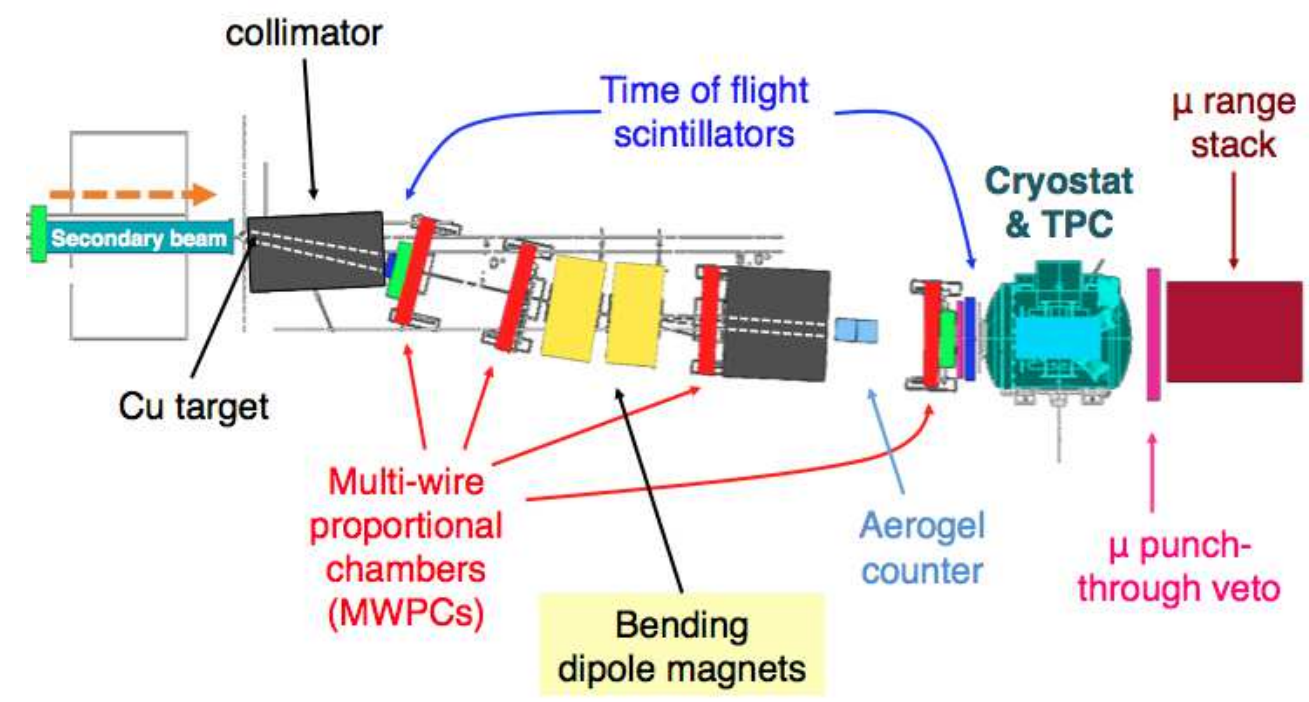

Figure 4.1: A schematic overhead view of LArIAT's beamline and TPC in the Meson Center at the Fermilab Test Beam Facility.

limited detail. The high-energy secondary beam is first impinged onto a copper $(\mathrm{Cu})$ target embedded in a steel collimator as illustrated on the left in the overhead view of the tertiary beamline shown in Figure 4.1. The collimator has a narrow opening at $13^{\circ}$ off-axis, allowing for outgoing particles emanating from the pion- $\mathrm{Cu}$ interactions to continue onward along the beamline. Particle trajectories are tracked by four wire chambers as they traverse the beamline and are bent by about $10^{\circ}$ in the dipole magnets. Momentum $(P)$ can then be calculated from this information, which, together with the particle time-of-flight (TOF), is used to determine particle mass and select particles for analysis [69]. Aerogel Cherenkov detectors and a muon range stack have the potential to help in further distinguishing $\pi$ from $\mu$ (which are close enough in mass to be indistinguishable in the mass selection), though data from these detectors have yet to be used in ongoing analyses.

\subsection{Cryogenic system}

The LArIAT cryostat, shown on the left in Figure 4.2, is composed of a cylindrical inner cryostat $(76.2-\mathrm{cm}$ in diameter and $130 \mathrm{~cm}$ in length) filled with purified LAr in which sits the time projection chamber (TPC). Surrounding this is a region of vacuum and layered 

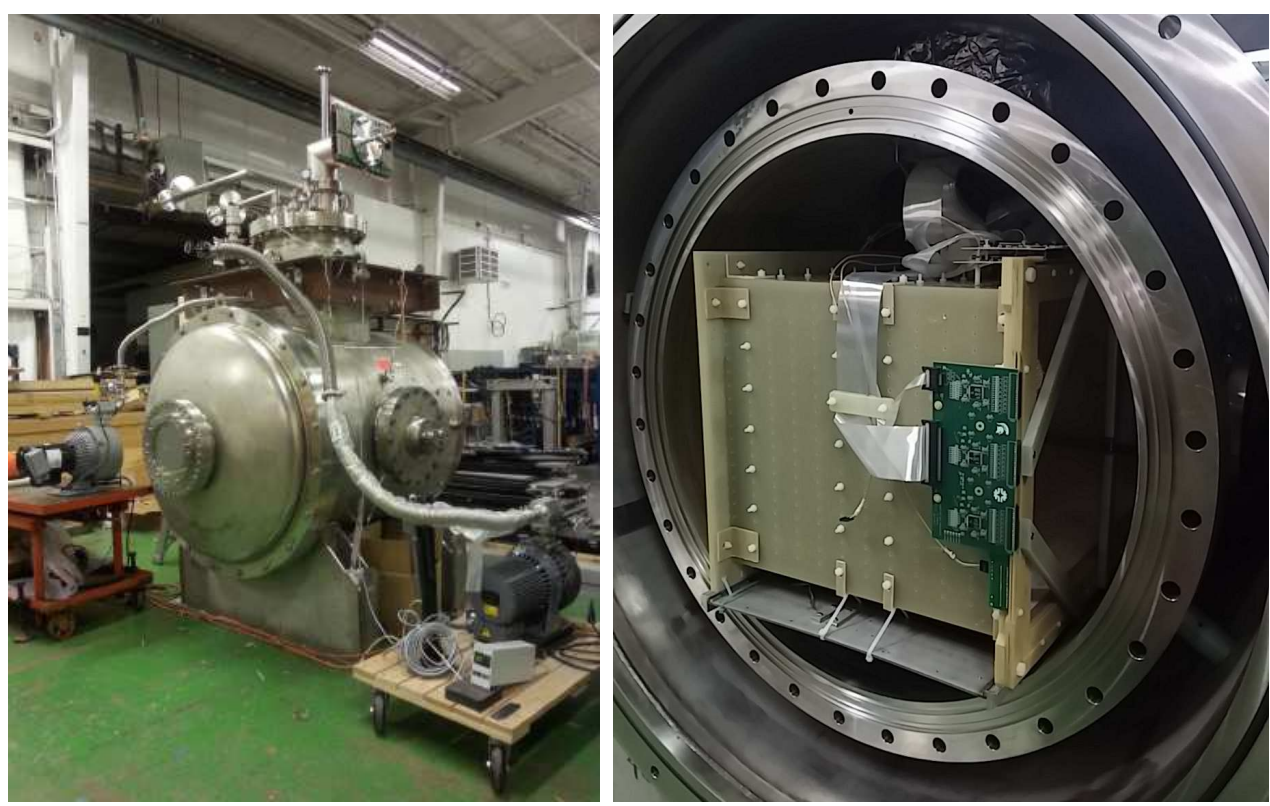

Figure 4.2: The fully-sealed LArIAT cryostat (left) and the TPC sitting inside the open inner cryostat (right).

aluminized mylar sheets to provide insulation for the inner cryostat. A wide neck protrudes from the top to allow for signal and high-voltage (HV) cables, and a side-flange with several high-voltage feedthroughs provide signal and HV to the region behind the wireplanes where the light collection system is mounted.

A commercial dewar of LAr sits outside the Meson Center enclosure. The LAr passes from the dewar to a purification system [71] consisting of several stages which, together, remove primarily water and oxygen $\left(\mathrm{O}_{2}\right)$ as well as small amounts of nitrogen $\left(\mathrm{N}_{2}\right)$. Electrons in LAr easily attach to water and $\mathrm{O}_{2}$ along their drift, so maintaining good LAr purity $(<100$ ppt) of these two contaminants is critical for ensuring electron drift lifetimes are long enough not to adversely affect signal integrity $\left(\tau_{e} \gtrsim 1 \mathrm{~ms}\right)$.

Filtered LAr is then piped into the inner cryostat, filling it up from the bottom. Argon level, temperature, and pressure are monitored using several sensors positioned at varying heights in the cryostat. Over time, LAr boils off and must be refilled several times per day during data-taking in order to maintain a level such that the TPC readout electronics are continually submerged [69]. 


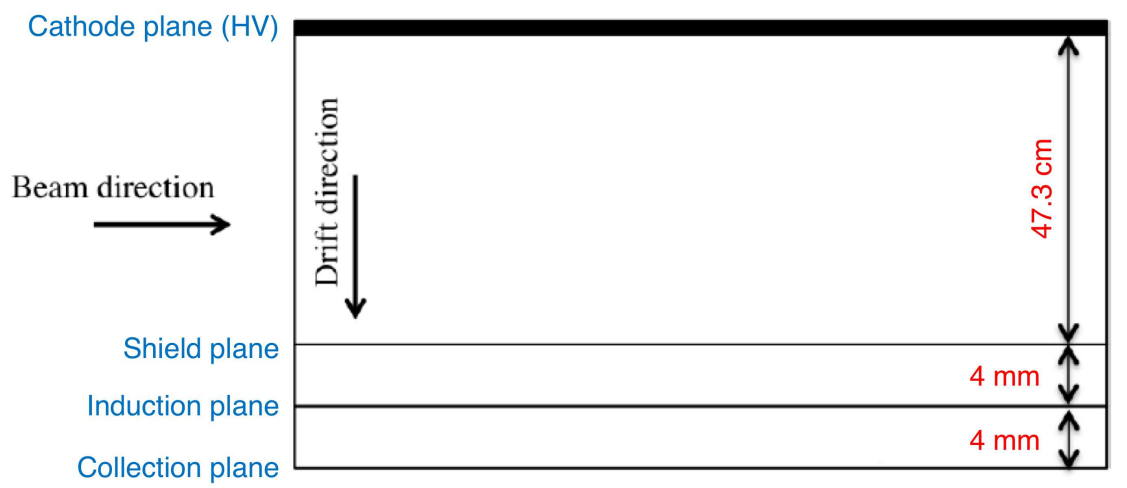

Figure 4.3: Schematic representation of the three drift regions in the LArIAT TPC.

\subsection{Time projection chamber}

The LArIAT TPC is a refurbished version of the TPC from ArgoNeuT which ran in the NuMI $\nu$ beamline from 2009-2010 [35, 36]. In accordance with the conventional TPC coordinate system for LArTPCs, the (secondary) beam axis determines the $\mathrm{Z}$ direction while Y describes the vertical dimension and X describes the "drift" coordinate parallel to the applied electric field. LArIAT's 170-liter liquid argon active volume has dimensions of $47.3 \mathrm{~cm}(x) \times 40 \mathrm{~cm}$ $(y) \times 90 \mathrm{~cm}(z)$.

LArIAT's TPC, shown on the right in Figure 4.2, is equipped with a total of three wire planes: a non-instrumented 225-wire shield plane, and 240-wire induction and collection readout planes. All planes have a wire pitch (or separation) of $4 \mathrm{~mm}$. Wires on the induction and collection planes are oriented at $\pm 30^{\circ}$ relative to the vertical direction. High voltages are applied to the cathode plane $(\approx-23.2 \mathrm{kV})$, shield plane $(-298 \mathrm{~V})$, induction plane $(-18.5 \mathrm{~V})$ and collection plane $(338 \mathrm{~V})$ to induce a uniform electric field of strength $\mathscr{E}=484 \mathrm{~V} / \mathrm{cm}$ parallel to the X-direction. This field strength is also measured in data from observed electron drift velocities for anode-cathode-piercing muon tracks [69]. The relative biasing between planes is set to enable electron transparency conditions on the shield and induction planes such that the drift electrons pass by these two planes and induce signals before being collected on the final (positively-biased) plane. Figure 4.3 illustrates the three distinct regions in the TPC across which electrons drift before finally being absorbed onto the collection plane. 
After their assembly and prior to their installation in the TPC, the wire planes were tested using simple electrical continuity tests and through measurements of the RC time constant following the injection of a $20 \mathrm{~Hz}$ square wave.

\subsection{Photon detection system}

We now describe LArIAT's system for collecting scintillation light, a critical ingredient to the analysis which will follow in subsequent chapters.

LArIAT is unique among existing LArTPCs in how it implements wavelength-shifting. As discussed in Chapter 3 , the scintillation photons from the decay of $\mathrm{Ar}_{2}^{*}$ are produced in the VUV regime $(\lambda=128 \mathrm{~nm})$. To match the spectral sensitivity of most photodetectors, which respond primarily to visible light, light collection systems in LAr typically rely on tetraphenyl butadiene (TPB) to down-convert the VUV scintillation photons into the blue-visible regime $(\lambda \approx 430 \mathrm{~nm})$. In LArIAT the active target volume is surrounded by wavelength-shifting material, a technique adapted from many scintillation-based dark matter experiments. The four inner walls of the TPC's field cage are lined with dielectric foils made of a polymeric layer of highly specular reflectivity. These foils have been evaporatively coated with a layer of the wavelength-shifting TPB. VUV photons produced by ionizing particles are wavelengthshifted at these foils and the re-emitted visible photons are subsequently reflected back into the active volume where they have a chance of being detected. Compared to more traditional methods where TPB is coated or suspended in front of the PMTs' windows - for example, as is done in MicroBooNE [24] - LArIAT's use of reflector foils increases the average light yield by nearly a factor of two and improves light yield uniformity throughout the active volume.

Each visible wavelength-shifted photon propagates through the LAr while undergoing much less scattering compared to the VUV light. A photon may continue reflecting off of the other foils until it is eventually detected by one of two cryogenic PMTs, a 76-mm Hamamatsu (HMM) and a 51-mm Electron Tubes Limited (ETL). Each PMT is suspended behind the wire planes within a PEEK support structure that screws into a side access 

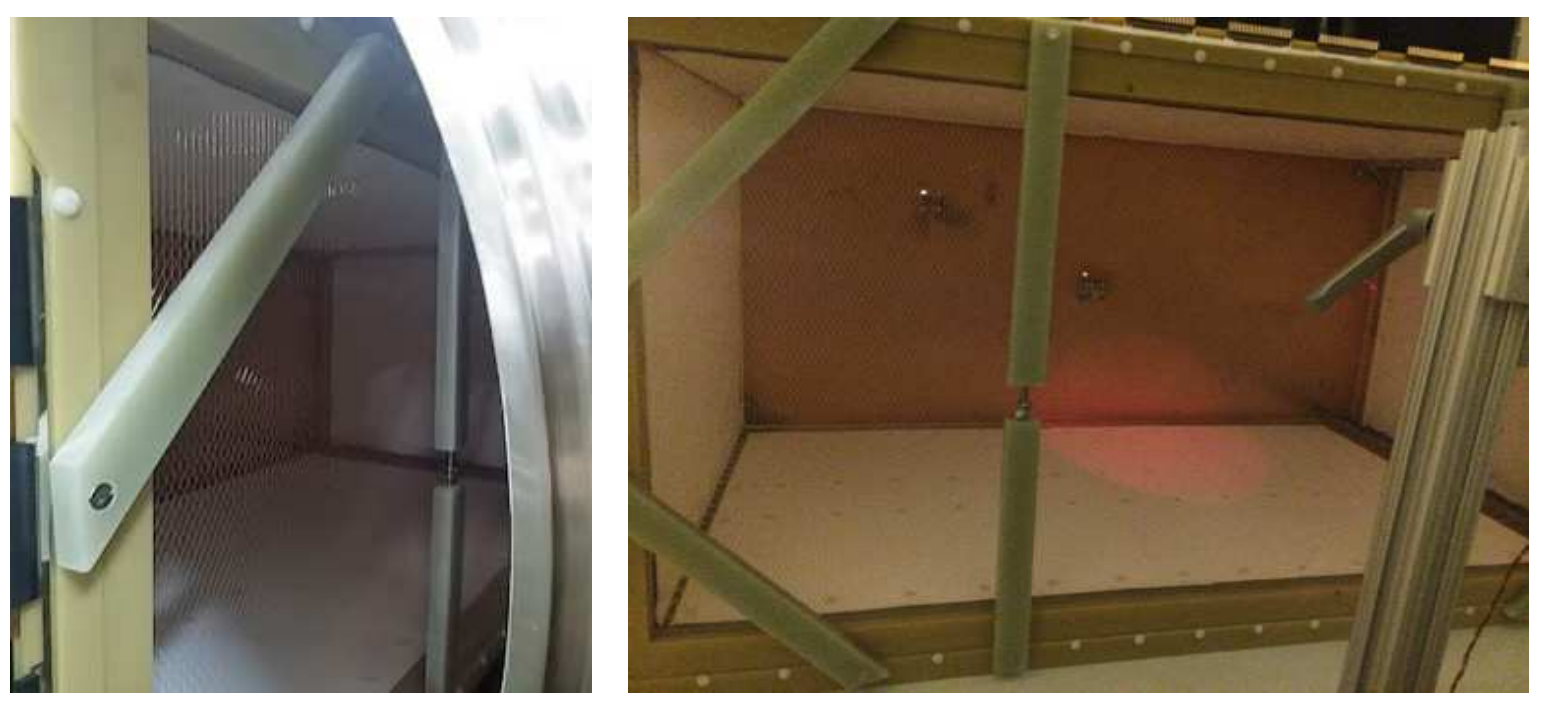

Figure 4.4: A view of the TPB-coated foils lining the field cage walls.

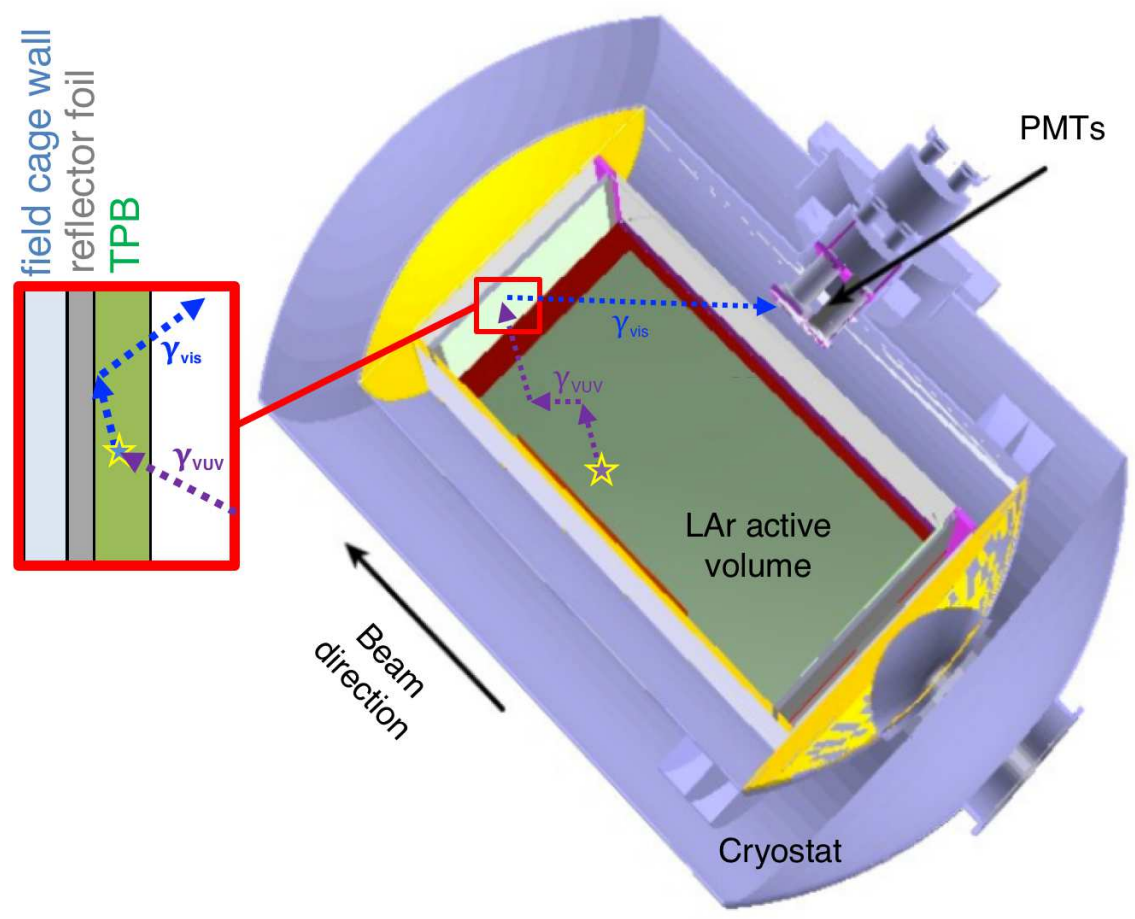

Figure 4.5: A cartoon schematic illustration of VUV light being wavelength-shifting along the TPB-coated reflecting foils. 

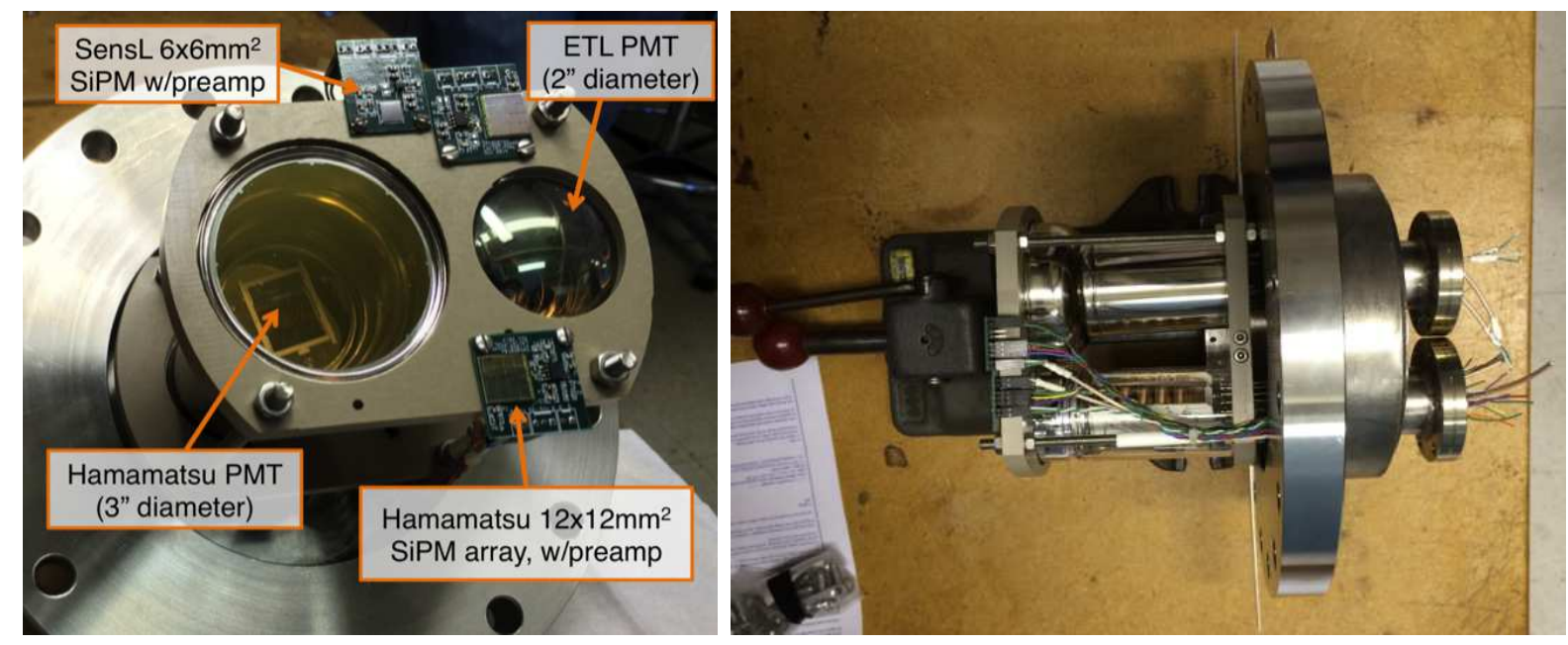

Figure 4.6: The light collection system mounted to the inner flange of the cryostat's side port prior to installation.

flange as see in Figure 4.6. The PMTs' windows are held parallel to the wire planes with about $5 \mathrm{~cm}$ of clearance. Both PMTs were designed for operation in cryogenic temperature, and were tested [73] and used successfully by the WArP Collaboration [66] in Gran Sasso, Italy. Their operating voltages in LAr range from $1.1 \mathrm{kV}$ to $1.5 \mathrm{kV}$, and they draw a current of about $100 \mu \mathrm{A}$ each. These devices emit low levels of radioactivity and have high photoelectron conversion efficiencies - about $29.5 \%$ for the Hamamatsu and $17.5 \%$ for the ETL when averaged over the visible TPB re-emission spectrum. The collection efficiency, or the probability of a photoelectron successfully progressing through the dynode chain, is 9095\% for the Hamamatsu and 75-80\% for the ETL [73]. Schematic diagrams for both PMTs' voltage dividers are presented in Figures 4.7,4.8, including two slightly different designs for the Hamamatsu that were used between the run periods [74].

Aside from the PMTs, several silicon photomultiplier (SiPM) arrays were incorporated into LArIAT's light collection system as well. These boards are shown in Figures 4.9 and 4.11 . Custom printed circuit boards (PCBs) displayed in Figures 4.10 and 4.12 were designed to amplify and read out the signals from the SiPMs, each of which were mounted to their board via a solder-reflow method. The PCBs include an on-board inverting transimpedance amplifier circuit which uses an OPA656 op-amp. There is a rising interest in using SiPMs for 


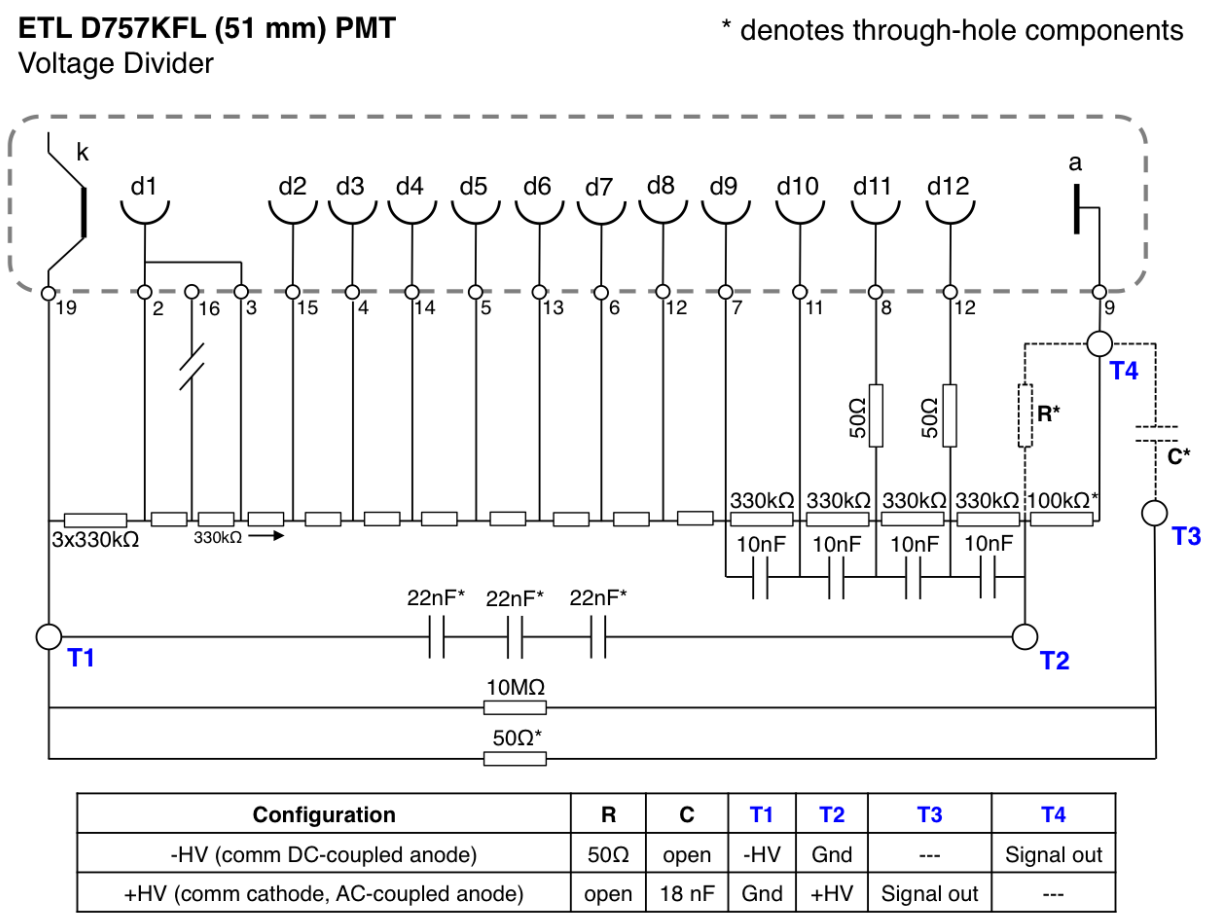

Figure 4.7: Voltage divider for the 2"-diameter ETL D757-KFL PMT.

Hamamatsu R-11065 (76 mm) PMT

* denotes through-hole components

Custom-made voltage divider

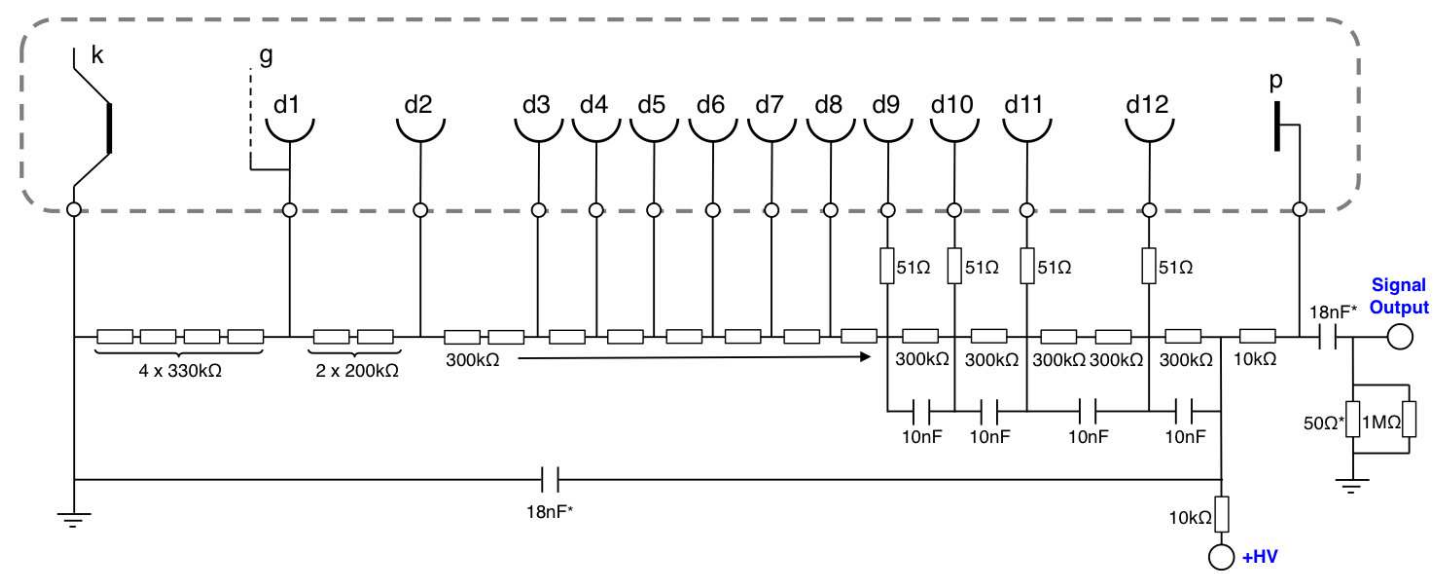

Figure 4.8: Voltage divider for the 3"-diameter Hamamatsu R-11065. 
light collection in LArTPCs for neutrino physics in place of conventional PMTs due to their lower operating voltage $(\approx 25 \mathrm{~V})$, smaller profile, and insensitivity to external magnetic fields. LArIAT's inclusion of these devices served as a "proof-of-principle" test that such on-board amplification circuits could be used to read out SiPMs in cryogenic temperature. However, given their small active areas $\left(6 \times 6 \mathrm{~mm}^{2}\right.$ and $\left.12 \times 12 \mathrm{~mm}^{2}\right)$ the SiPM boards contribute negligibly to the total light yield. Therefore, data from these SiPMs were not used in the analysis presented in this thesis.

There were several important differences in the light system between LArIAT's run periods which must be noted:

- In Run I, both PMTs were configured for negative bias with the DC-coupled collection anode held at ground and the photocathode at high voltage (HV). In Run II, to reduce noise and interference induced on the wire planes due to the proximity to the charged PMT photocathodes, the PMT voltage dividers were modified for operation in positive bias mode; in this configuration, the photocathode was grounded and the anode equipped for AC-coupled readout.

- For Run I and much of Run II, only the ETL PMT was powered due to a malfunction in the Hamamatsu voltage divider. Midway through Run II, the Hamamatsu was repaired. The Run II dataset used in this analysis had both PMTs fully functional, and will be referred to as Run IIB to distinguish it from the first part of the Run II data-taking period where only the ETL PMT was operational.

- Prior to the start of Runs IIA and IIB, a translucent film of a TPB/polystyrene solution 11 was added to the window of the ETL PMT (see Figure 4.13). This TPB film allowed some of the VUV scintillation light from Ar to down-convert directly at the face of the PMT instead of at the coated reflector foils. The decision to coat the ETL

1. The TPB coating was made by dipping the PMT's window twice in a solution of $100 \mathrm{~mL}$ toluene, $2 \mathrm{~g}$ polystyrene, and $800 \mathrm{mg}$ (600 mg) of TPB powder for Run IIA (IIB). 

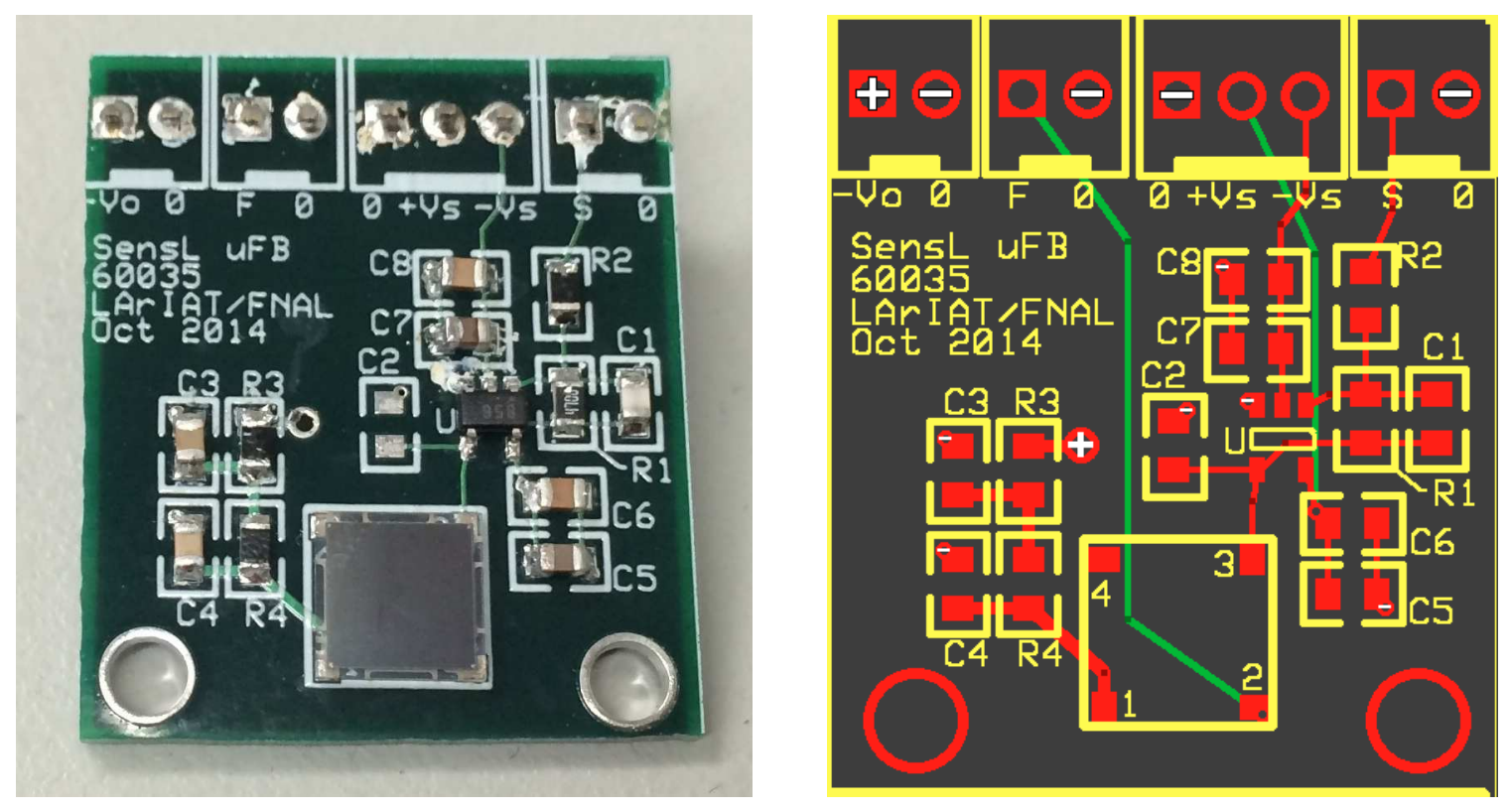

Figure 4.9: Custom SiPM readout board for the $6 \times 6 \mathrm{~mm}^{2}$ SensL MicroFB-60035.

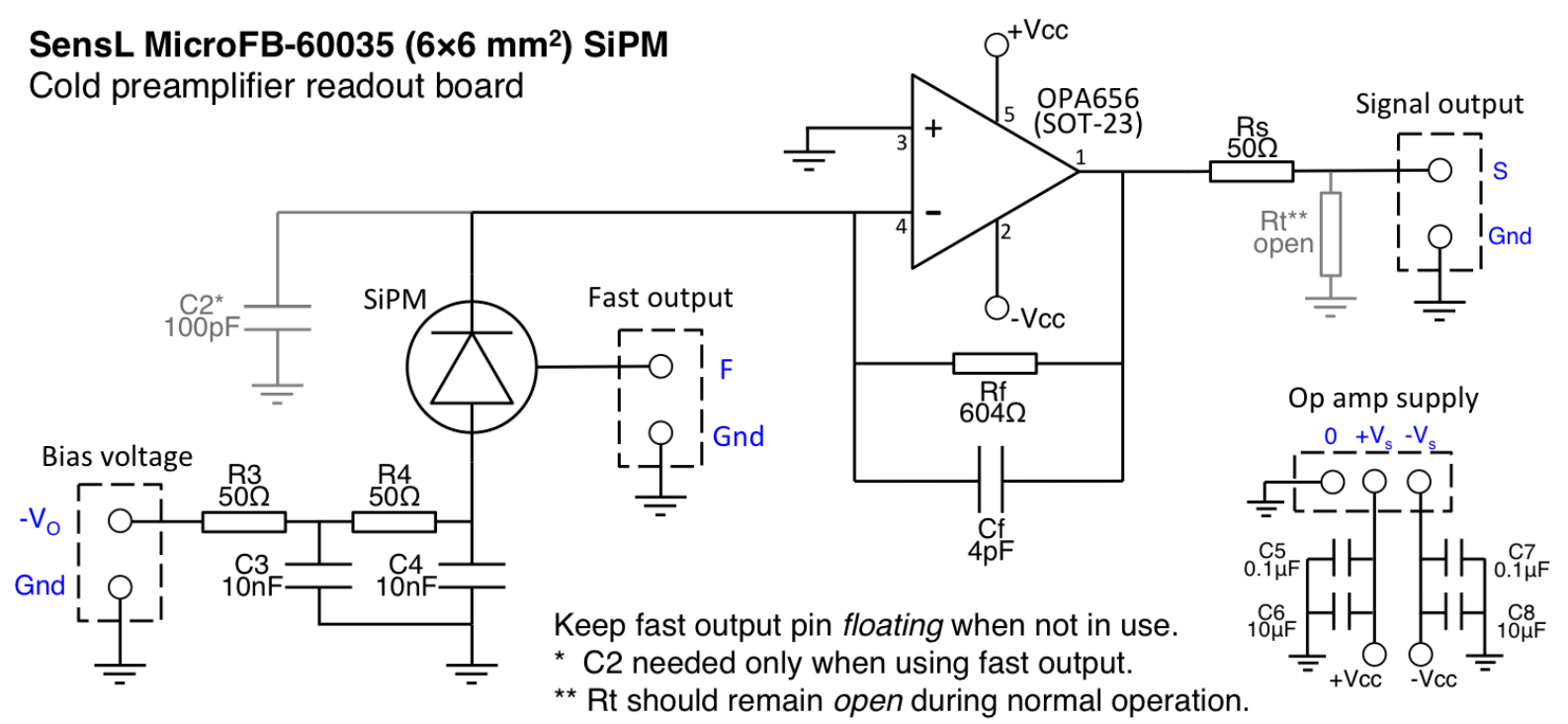

Figure 4.10: Schematic board diagram for the SensL MicroFB-60035 chip with an on-board inverting transimpedance amplifier circuit using an OPA656 op-amp. 

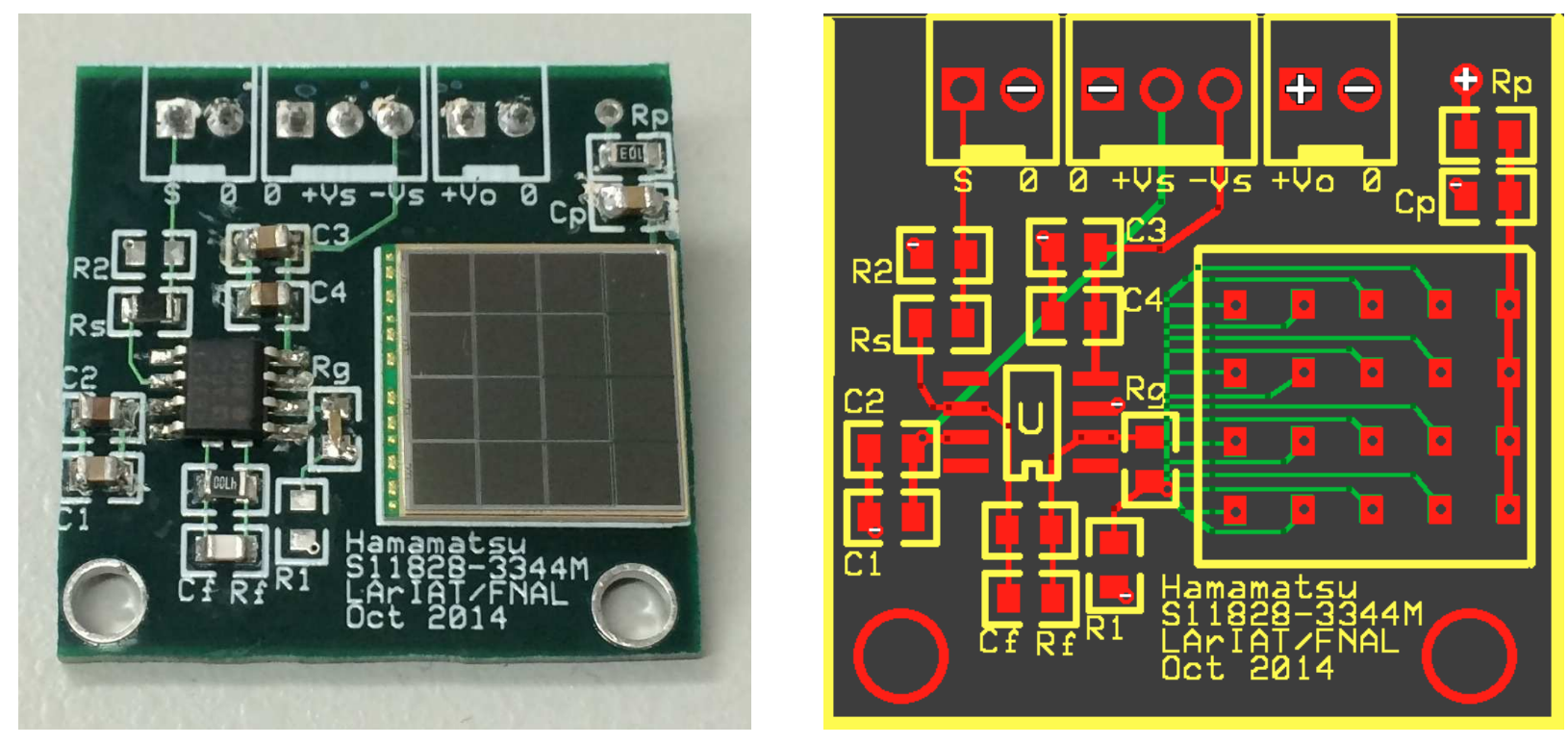

Figure 4.11: Custom SiPM readout board for the $12 \times 12 \mathrm{~mm}^{2}$ Hamamatsu S11828-3344M MPPC array.

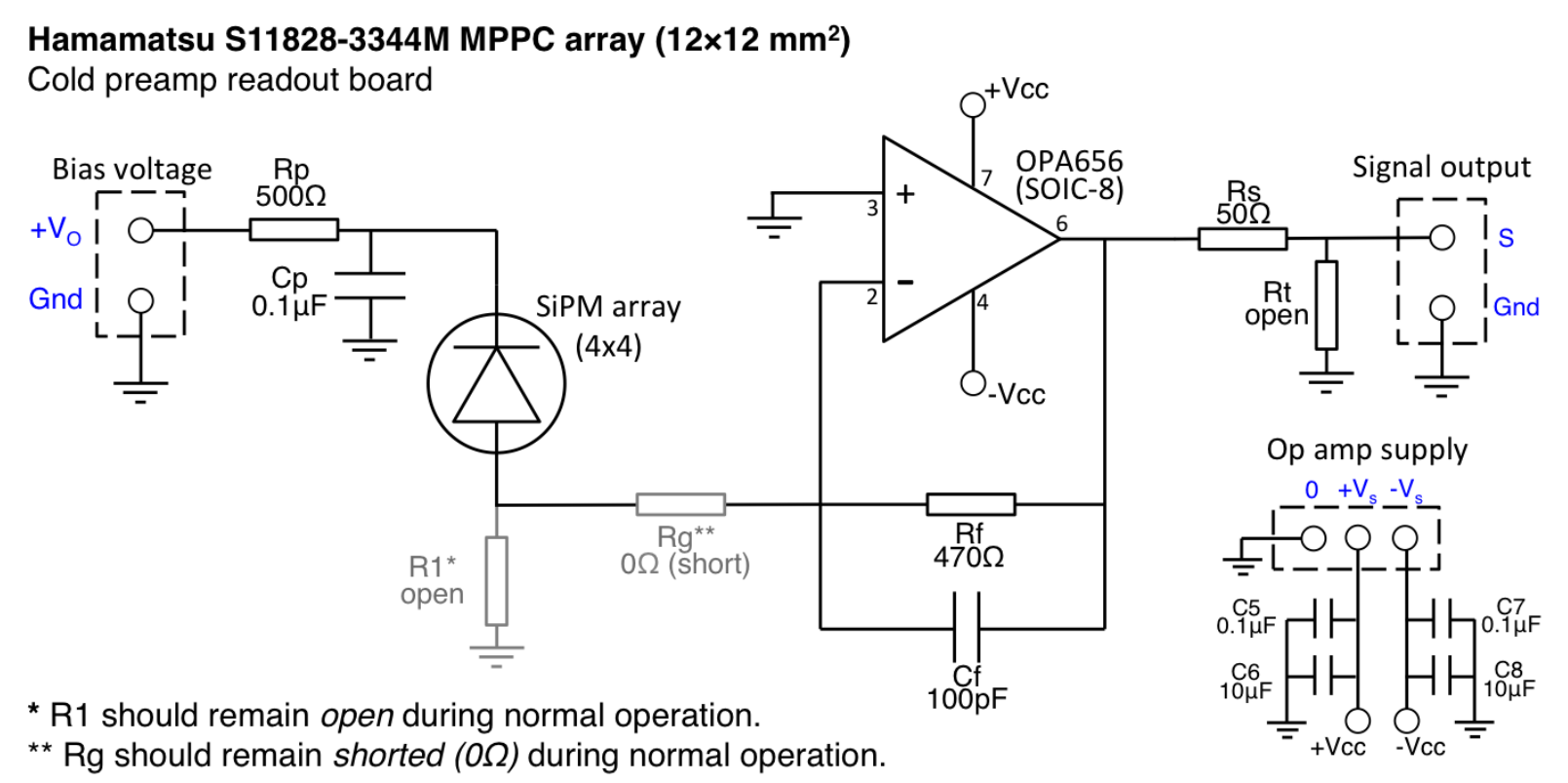

Figure 4.12: Schematic board diagram for Hamamatsu S11828-3344M MPPC array with an on-board inverting transimpedance amplifier circuit using an OPA656 op-amp. 


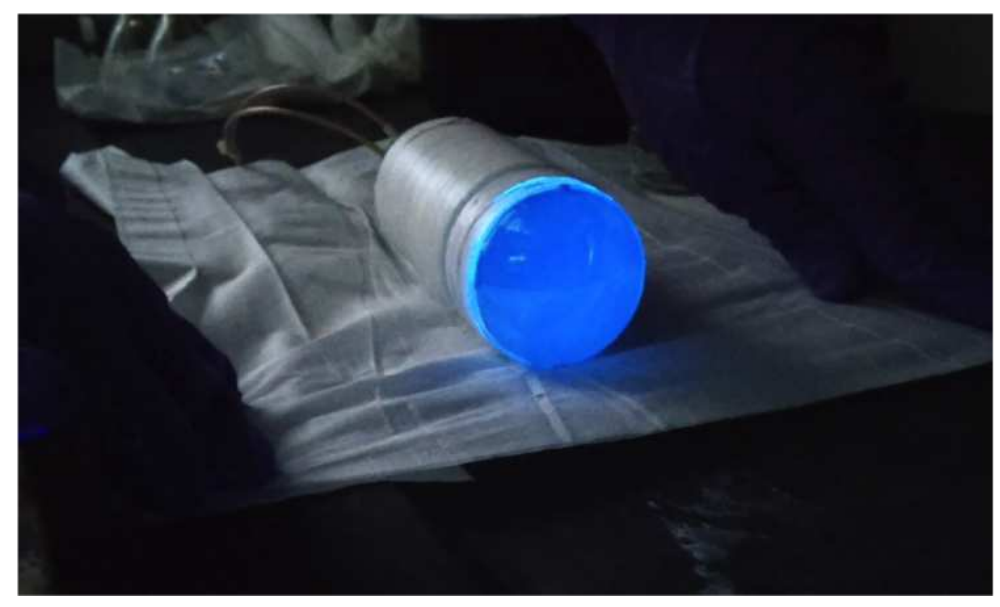

Figure 4.13: The ETL PMT illuminated under a UV lamp after application of TPB film. Visible re-emission from TPB induced by impinging UV radiation causes it to glow.

in TPB was motivated by potential scintillation timing studies which it enabled, and was not directly related to the Michel electron analysis described in this thesis.

\subsection{Data acquisition}

Readout of the TPC and photodetector signals (along with the beamline detectors, which are not relevant to this thesis) is triggered using the CAEN V1495, a configurable coincidence module capable of handling 16 logic inputs. User defined patterns are set and the inputs (sampled at $100 \mathrm{MHz}$ ) are checked for any matches to these patterns. If the inputs match a pattern for two consecutive samples, a readout request is generated.

Ionization drifted to the wires produces relatively small electrical signals which must be amplified. All 480 wire channels are read out through cold amplifier motherboards hosting ASICS (LArASICS) [76] which are mounted along the outer frame of the wire planes submerged in LAr. Signals are then routed through warm receiver/driver cards and across $\sim 10 \mathrm{~m}$ cables to CAEN V1740 digitizers where they are sampled every 128 ns with 12-bit resolution during readout [69]. A 3072-sample waveform is saved per wire totaling $393 \mu \mathrm{s}$ (the total drift time for an event is $320 \mu \mathrm{s}$ ). Typical analog signals from MIPs are roughly $90 \mathrm{mV}$ in amplitude which corresponds to about 180 ADC after digitization. 


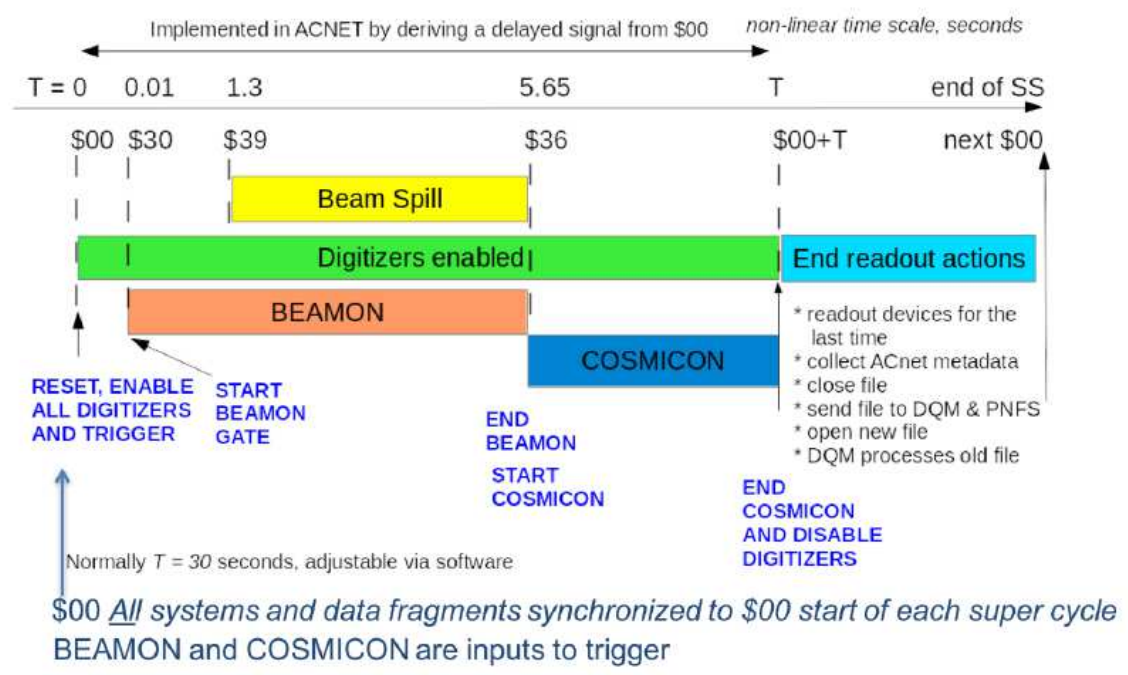

Figure 4.14: The Meson Center beam supercycle. Cosmic triggers are active in the 24-second COSMICON window following each beam spill.

Signals from the photodetectors are first routed to a linear fan-in/fan-out NIM module where analog copies of the signals are made. One copy of each PMT signal is sent to a CAEN V1751 digitizer and sampled every $1 \mathrm{~ns}(1 \mathrm{GHz})$. Other copies of the signals are used in light-based trigger logic described in Section 4.7 to follow. Optical waveforms 28672 samples in length $(28.7 \mu \mathrm{s})$ are saved per channel for each triggered event.

LArIAT receives 4.2 seconds of MI-extracted beam once every 60.5 seconds as part of Fermilab's accelerator complex "super cycle" as shown in Figure 4.14. Following this period of beam, LArIAT triggers on cosmic muon events for a period of about 24 seconds. At the time of LArIAT's conception, this cosmic readout window was intended to include only events triggered by a pair of scintillator paddles on either side of the TPC within the enclosure which are arranged to select only muons that cross the long diagonal of the TPC. Such events are useful in measuring the electron attenuation lifetime for calibration purposes since they span the full drift length and are assumed to have a MIP-like energy deposition density through their path (see Chapter 6). However, it was soon realized at the start of Run I that additional triggers could be implemented using the optical signals. Several scintillation-based trigger patterns were developed and tested over LArIAT's data-taking periods, including one to 
select stopping-and-decaying cosmic muons as described in the next section.

\subsection{Light-based Michel electron trigger}

A hardware-level trigger was set up to collect events where a cosmic muon stops inside the TPC and decays to a Michel electron after some time delay. This trigger is represented schematically in Figure 4.15. Copies of both PMT signals are first amplified x10 in a NIM amplifier module and then discriminated at thresholds ranging 80-140 mV, which corresponds to discrimination at 8-14 $\mathrm{mV}$ on the original unamplified signals. This amplification is necessary in order to extend the trigger sensitivity to lower signal voltages since the discriminator module has a minimum threshold of $\approx 50 \mathrm{mV}$. A coincidence of these discriminated signals produces the logic signal LARSCINT. The LARSCINT signal opens a 7- $\mu$ s-wide gate and, by nature of the gate generator module, imposes a "dead time" during which subsequent LARSCINT signals will not open more gates. A second LARSCINT produced in coincidence with this gate produces the $M I C H E L$ signal. In order to prevent accidental mis-fires in which a pulse coincides with itself, the coincidence gate is delayed by 250-300 ns. An example oscilloscope screenshot of this trigger in action is shown in Figure 4.16. Starting in Run II, unamplified copies of the PMT signals were discriminated at $180 \mathrm{mV}$ to use as a veto on events that might saturate the full $200 \mathrm{mV}$ dynamic range of the CAEN 1751 digitizer.

Much time was spent experimenting and optimizing this trigger by viewing events in an oscilloscope in the Meson Center enclosure. An example oscilloscope readout trace is shown in Figure 4.16. The configuration was tweaked a number of times over the course of LArIAT's run time to test different schemes. The specific configurations used to collect the Michel electron samples used in our analysis is described in Chapter 8.

Michel electron candidate events were collected at a rate of 1-3 $\mathrm{Hz}$ depending on the run period. These events were a mix of true Michel events and accidental background events that faked the delayed double-pulse. An event display of a Michel electron candidate is displayed in Figure 4.17 . 


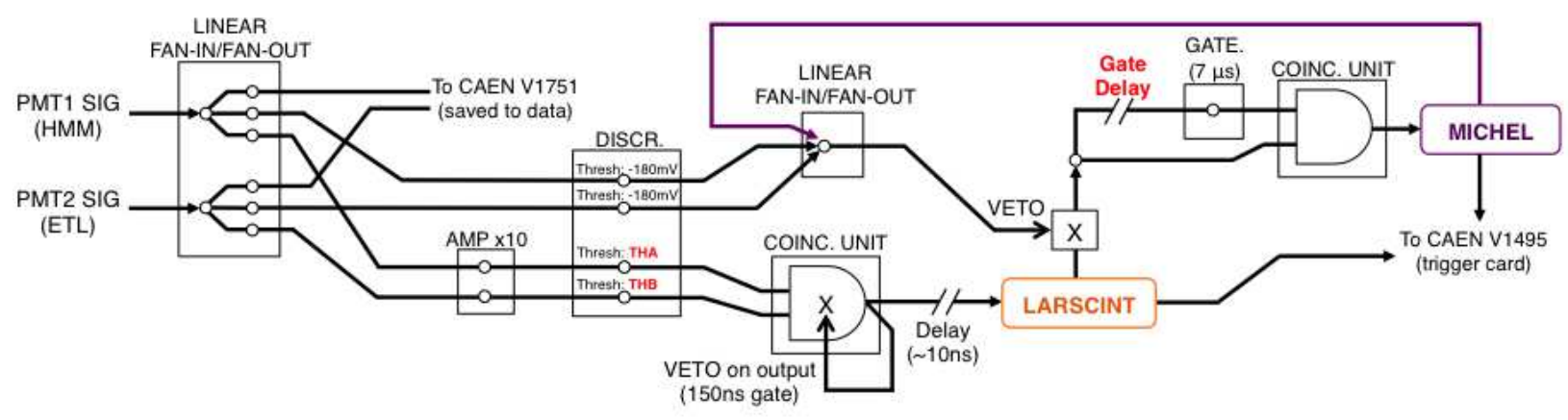

Figure 4.15: Schematic diagram of the logic used to make two of the light-based trigger signals in LArIAT, LARSCINT and MICHEL [74]

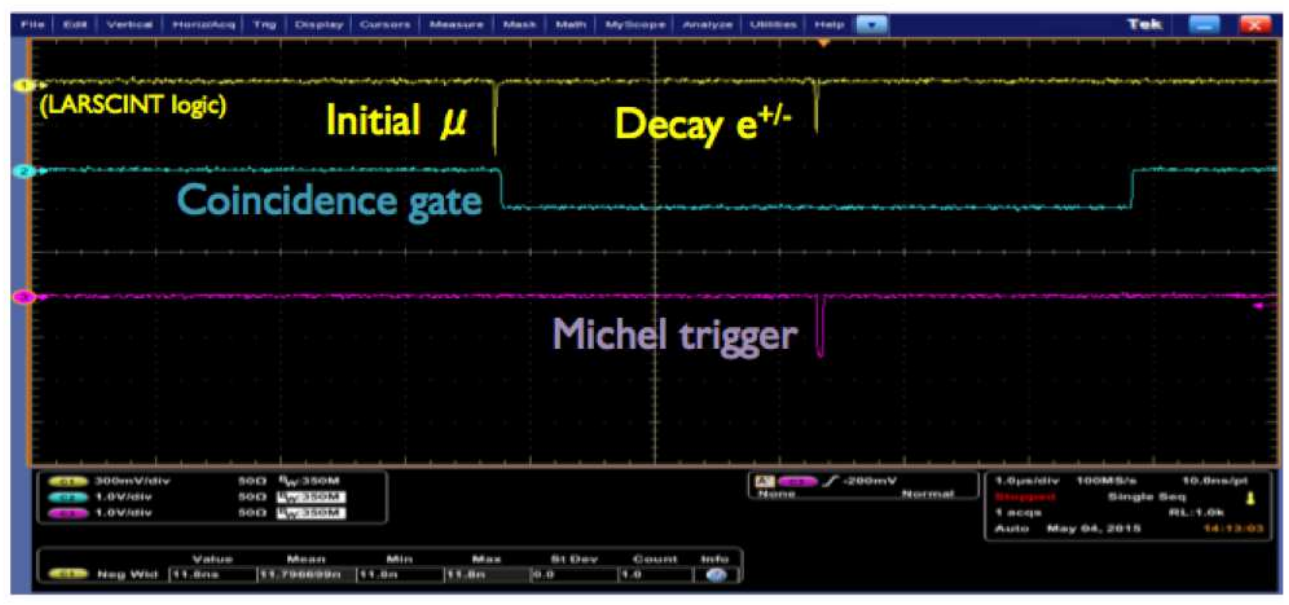

Figure 4.16: An oscilloscope screen capture demonstrating the trigger for a cosmic Michel decay candidate captured using the light-based trigger. 


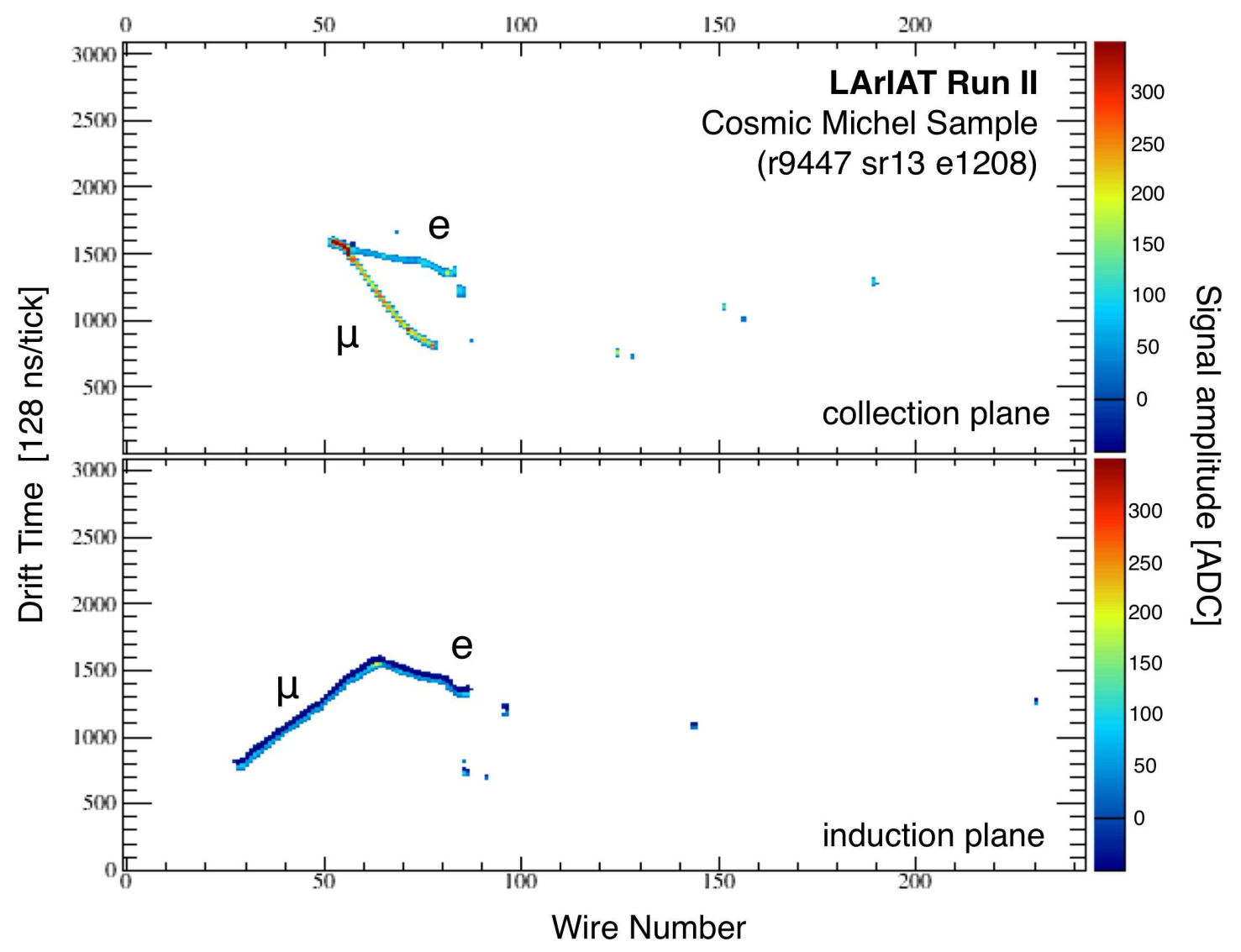

Figure 4.17: Raw data event of a stopping muon with its decay electron from the Michel electron light-based trigger. Vertical columns of pixels represents the raw signal collected on wires. The color scale indicates wire signal amplitude at each time-tick. 


\section{5}

\section{Reconstruction of Michel Electrons}

This chapter covers the reconstruction of the sample of low-energy electrons obtained from the light-based Michel electron trigger. First the physics of muon decay are reviewed and the specific data samples are introduced for both Run I and Run IIB. Then the steps involved in reconstructing physical quantities from the raw data are outlined using both the charge and optical data. Finally, in order to validate the sample, the muon decay time spectrum is reconstructed and used to extract a measurement of the negative muon capture lifetime/probability as well as the cosmic muon charge ratio.

\subsection{Overview of muon decay and capture}

Muons undergo $\beta$-decay with a well-measured vacuum lifetime of $\tau_{\text {free }}=2.197 \mu \mathrm{s}$ [79], with each decay producing either a positron or an electron and two neutrinos:

$$
\mu^{+(-)} \rightarrow e^{+(-)}+\nu_{e(\mu)}+\bar{\nu}_{\mu(e)}
$$

In the rest frame of the muon, the kinematic phase space available to the final products of this decay give the outgoing $e^{+(-)}$a distinctive energy spectrum with a sharp cut-off at $E_{e}=m_{\mu} / 2 \approx 53 \mathrm{MeV}$. Electrons from muons that lose enough energy to come to a stop in a medium before decaying are referred to as Michel electrons. A measurement of the Michel energy spectrum is shown in Figure 5.1 [77].

Muons that come to a rest in media behave differently depending on their charge sign. As a $\mu^{-}$slows down and approaches rest, it becomes bound to a nucleus due to the attractive Colulomb potential and immediately cascades down to the $1 \mathrm{~S}$ atomic orbital. Bound $\mu^{-}$ can still undergo decay, though the energy of the emitted electron is smeared slightly from Doppler shift effects due to the inherent velocity of the $\mu^{-}$in orbit. This effect softens the 

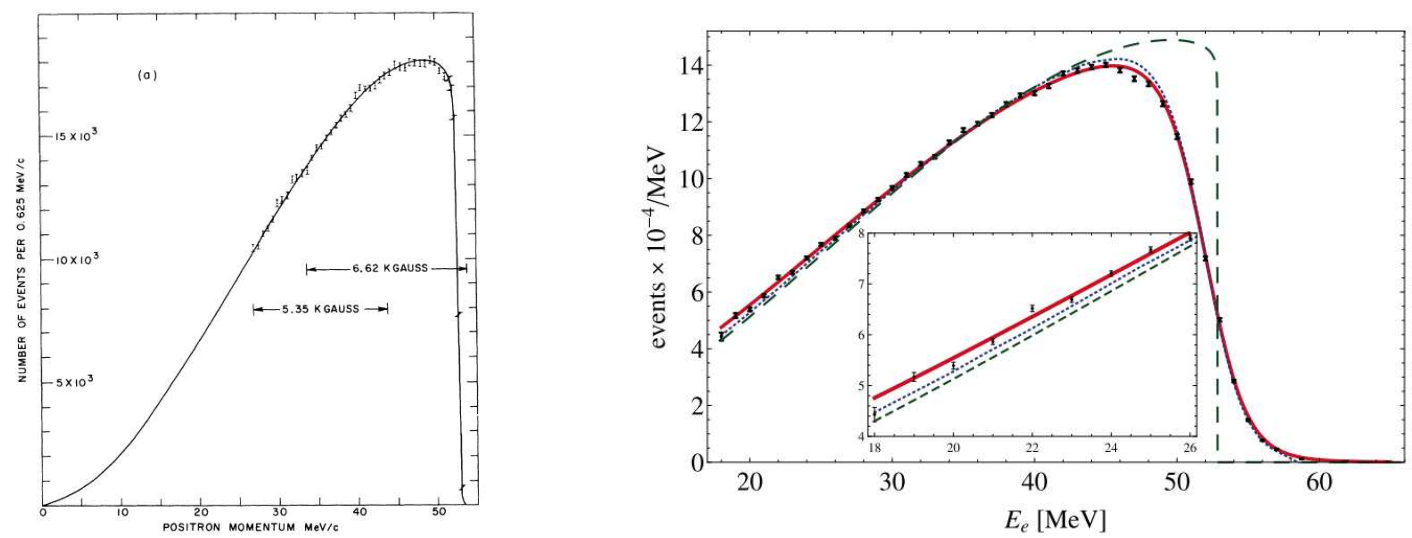

Figure 5.1: Positron energy distribution from decay-at-ret $\mu^{+}$at the Columbia University cyclotron at the Nevis laboratories (left) [77]. On the right is the energy spectrum for bound $\mu^{-}$decaying in-orbit. Data from the TWIST experiment (black points) are compared to theoretical curves with (solid red line) and without (dotted blue) radiative corrections. The dashed green line represents the electron spectrum from free $\mu^{+}$that stop and decay 82 .

$53 \mathrm{MeV}$ edge in the Michel spectrum [82], and also slightly reduces the rate of decay for the bound $\mu^{-}$. Given the relatively small Bohr radius of a bound muon in its lowest orbital state $\left(\mathrm{a} \approx 0.005 a_{0} \approx 0.0029\right.$ angstrom $)$, the muon's wavefunction partially overlaps with that of the nucleus. This enables a nuclear capture process to take place, $\mu p \rightarrow \nu_{\mu} n$. The probability of capture competes with that of decay, resulting in an effective $\mu^{-}$lifetime,

$$
\tau_{\mu-}=\left(\frac{1}{\tau_{c}}+\frac{Q}{\tau_{\text {free }}}\right)^{-1}
$$

where $\tau_{c}$ is the lifetime of the capture process and Q (=0.988 for Ar [80]) is the Huff factor, a minor corrective term to account for the reduction in decay rate for bound $\mu^{-}[81$.

Theoretical predictions based on the Primakoff formula [80] give a $\mu^{-}$-Ar capture lifetime of $\tau_{c}=851 \mathrm{~ns}$, which translates to an effective predicted $\mu^{-}$lifetime $\tau_{\mu^{-}}=615 \mathrm{~ns}$. Measurements by Suzuki and Measday [80] show $\tau_{c}=833 \pm 55 \mathrm{~ns}\left(\tau_{\mu-}=606 \pm 29 \mathrm{~ns}\right)$. A more recent measurement [83] in LAr showed $\tau_{\mu-}=616.9 \pm 6.7 \mathrm{~ns}$. 


\subsection{The Michel electron data samples}

Specific data-taking periods were selected from Run I and Run IIB during which the Michel trigger was functioning optimally. These are summarized in Table 5.1. Each dataset is defined by a curated range of runs with additional requirements on PMT voltages and TPC readout status to ensure good quality data. Care was taken to exclude runs taken during two specific periods in Run IIB during which the electric field was varied for test runs, and when the cryostat was drained and for argon filter regeneration.

Since both PMTs were operational in Run IIB, the scintillation logic signal was taken as the coincidence of both discriminated PMT signals. This allowed the discrimination threshold for the ELT PMT to be lowered in Run IIB without significantly adding to the number of noise-induced triggers.

Table 5.1: Summary of the nominal data samples used in this analysis.

\begin{tabular}{|c|c|c|c|c|}
\hline Datasets & Date Range & $\begin{array}{c}\text { Effective } \\
\text { trig. thresholds }\end{array}$ & $\begin{array}{c}\text { Trigger } \\
\text { gate delay }\end{array}$ & Notes \\
\hline Run I & $06 / 18 / 2015-06 / 29 / 2015$ & ETL: $-16 \mathrm{mV}$ & $300 \mathrm{~ns}$ & $\begin{array}{c}\text { ETL PMT only, } \\
\text { Negative HV mode }\end{array}$ \\
\hline Run IIB & $06 / 07 / 2016-06 / 22 / 2016$ & $\begin{array}{c}\text { HMM: }-14 \mathrm{mV} \\
\text { ETL: }-7 \mathrm{mV}\end{array}$ & $250 \mathrm{~ns}$ & $\begin{array}{c}\text { Positive HV mode, } \\
\text { Overshoot in HMM }\end{array}$ \\
\hline
\end{tabular}

\subsection{Charge reconstruction}

We now describe the stages in the procedure in which raw wire plane signals are used to reconstruct collections of charge deposits which belong to the decaying Michel electron. First, wire signals pass through a 1D deconvolution using Fourier transform techniques. Pulses are then identified and tagged as "hit" objects containing some amount of integrated charge arriving at some time. Hits are then clustered and information from both planes is used to form 3D tracks from line-like hit patterns. A custom clustering technique is then carried out which identifies hits belonging to the Michel electron. These stages are outlined in more detail below. 
Reconstruction is performed offline using LArSoft [78], a detector-generic suite of algorithms and modules written primarily in $\mathrm{C}++$, tailored for common LArTPC-specific data processing tasks.

\subsubsection{Wire signal deconvolution and filtering}

The measured output signal along a wire, $y(t)$, is a convolution of some impulse response $h(t)$ and the actual (unknown) signal generated by the collected or induced charge $x(t)$, along with some electronic noise component $n(t): y(t)=(x(t) * h(t))+n(t)$. The impulse response itself is a convolution of (a) the electronics response inherent to the readout electronics, shown in Figure 5.2, and (b) the expected plane-dependent field response as charge approaches and either passes by or collects on the wires. Signals on the collection plane will appear unipolar, whereas signals on the induction plane will have a bipolar shape; the field response functions characterize this effect. Figure 5.3 illustrates these field response functions.

A series of signal deconvolution algorithms are applied to the waveforms from both planes to reverse the detector effects and restore the true distribution of arriving charge, $x(t)$ [84]. While additional filtering is sometimes applied to LArTPC wire signals to remove noise and wire cross-talk, the signals in LArIAT are sufficiently clean and no filtering is performed.

\subsubsection{Hit-finding}

The deconvolved signals are fed to an algorithm which searches for pulses or regions of interest and attempts to fit them to Gaussian functions. A multiplet of fits is attempted if a single Gaussian doesn't sufficiently model the shape as seen in the middle plot in Figure 5.4 . When a pulse is successfully fit, each of the component Gaussians is used to create a hit object. The hit's properties are taken from parameters from the fit, including the hit charge which is simply the integral of the Gaussian. Each hit is therefore characterized by an arrival time and width (in time-ticks), and total charge (in ADCs) [84]. 


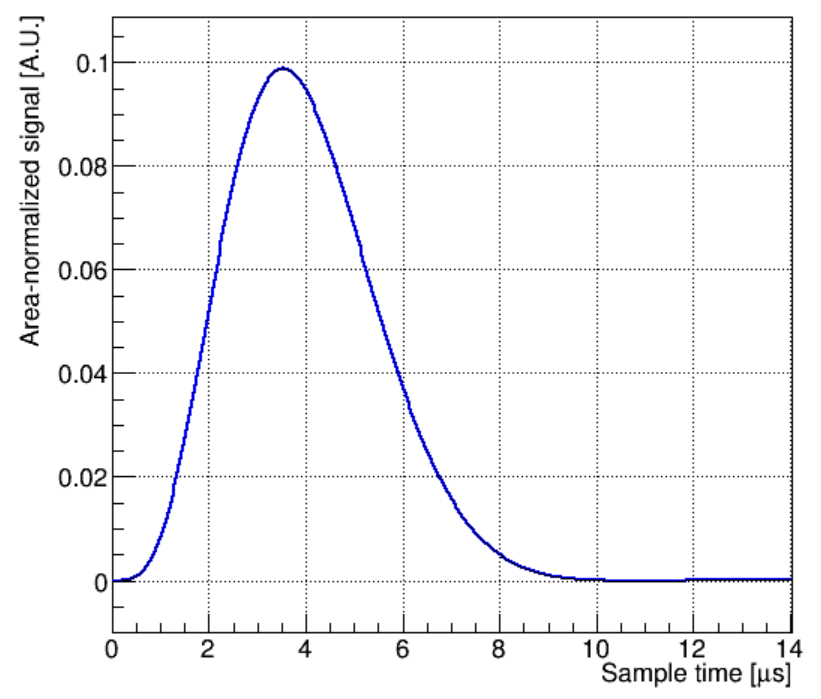

Figure 5.2: The electronics response of the wires as a function of time (arbitrarily-normalized) from BNL SPICE simulation of the LArIAT electronics.

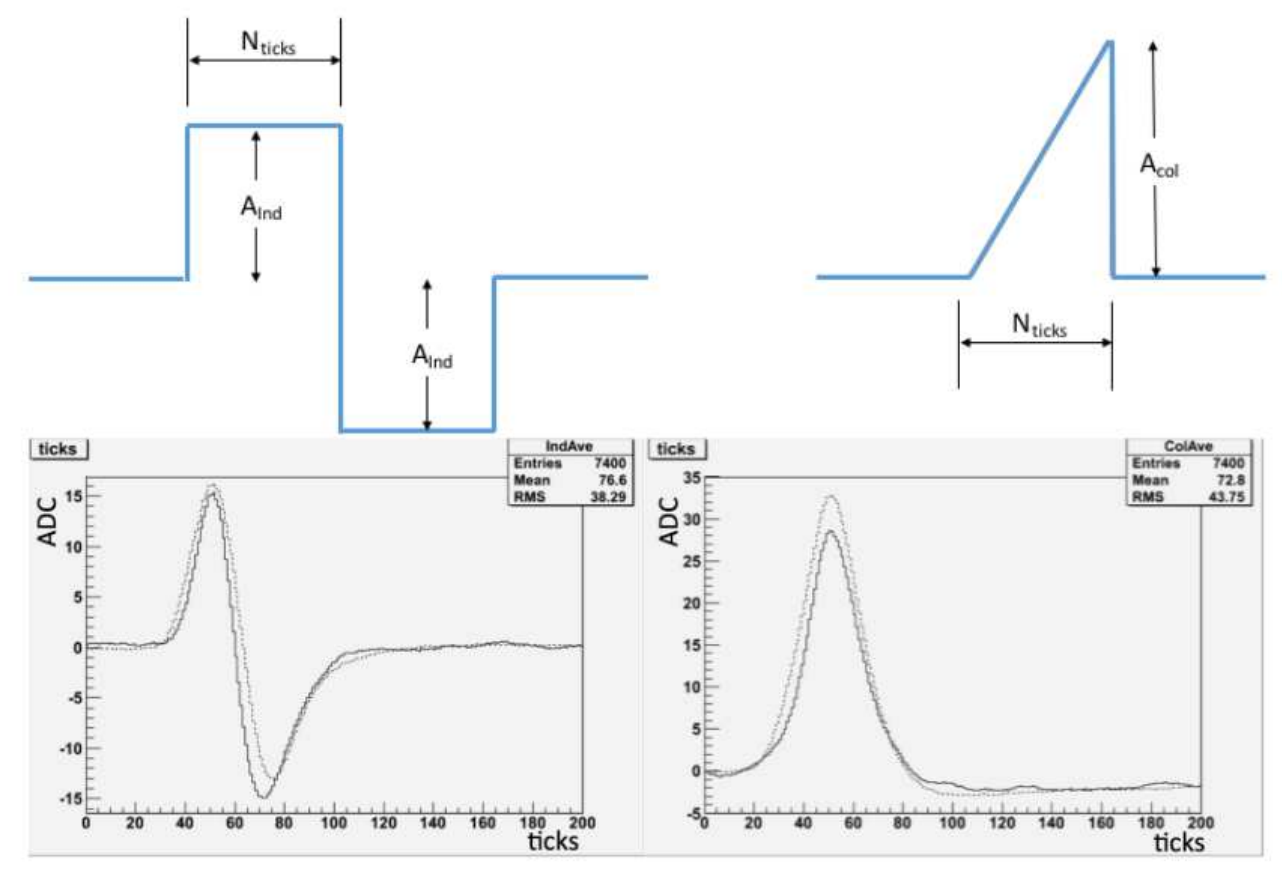

Figure 5.3: Simplified representations of the field response function (top) for the induction (left) and collection (right) plane wires. The bottom plots show real detector signals in solid lines and the simulated signals in dotted lines [84]. 
Each hit's time $(t)$ is corrected based on offsets to account for the pedestal region of the readout (i.e., samples prior to the sample corresponding to the trigger time), as well as the anticipated drift time between planes. We then map the hits into a 2D space defined by a wire coordinate $(W)$ and a drift coordinate $(X)$,

Wire coordinate: $W= \begin{cases}(\text { wire number }) \times 0.4 \mathrm{~cm}, & \text { collection plane } \\ (\text { wire number }-240) \times 0.4 \mathrm{~cm}, & \text { induction plane }\end{cases}$ Drift coordinate: $\quad X \quad=t \times v_{e}$,

where $0.4 \mathrm{~cm}$ is the spacing between adjacent wires and $v_{e}$ is the electron drift velocity. Note that care is taken to take into account the number convention for the wire planes which assigns wires 0-239 to the collection plane and wires 240-479 to the induction plane. We will refer to this wire-drift coordinate space as $\mathrm{W}-\mathrm{X}$ space moving forward.

\subsubsection{Charge clustering and $3 D$ track reconstruction}

Line-like groups of hits are identified using a trajectory-based clustering algorithm [85]. This algorithm steps along collections of hits in a 2D wire-time space for each plane and creates trajectory points by sequentially adding nearby hits, updating the direction of the trajectory along the way. Whether or not a new hit gets added to the trajectory depends primarily on the charge of the hit compared to the average of recently-added hits; how well the hit aligns with the current trajectory; and the angle between the two lines formed from a small collection of hits before and after the hit being considered. All the hits added to a trajectory are then aggregated into a single cluster.

The collection of $2 \mathrm{D}$ clusters from both wire planes are then provided as inputs to a $3 \mathrm{D}$ track-finding algorithm [86. To do this, a 3D trajectory is determined such that it minimizes a measure of the distance between its 2D-projections onto wire planes and the actual 2D hits 
observed for all wire planes simultaneously. The result is a collection of 3D track objects, each characterized by some number of $x y z$-points or "space points."

\subsubsection{Identification of muon/electron boundary}

The signal processing and reconstruction stages up to now are generally applicable to nearly all kinds of LArTPC events. We now turn to several hit-grouping techniques that are tailored specifically for the Michel electron topology.

In this first stage, we intend to cluster together all the hits belonging to both the incoming muon and its decay electron, forming a single contiguous cluster on both planes. To do this, we require a "seed" hit from which to begin the clustering. The seed hit is naturally taken to be the hit corresponding to the start of the stopping muon track where the muon enters the TPC. First, the 3D tracks in the event are evaluated and a stopping muon-candidate track must be identified in the event. A fiducial margin of $1.5 \mathrm{~cm}$ around the active rectangular volume is defined, and a track is tagged as stopping if its total length exceeds $5 \mathrm{~cm}$ and one of its nodes (endpoints) falls outside of this fiducial volume while the other node falls within it. There can be any number of passing or contained tracks, but if there is exactly one stopping track, it is tagged as the candidate muon and subsequent clustering can proceed.

A proximity-based clustering algorithm, designed to crawl along trails of charge in W-X space starting from the muon entrance point, is now used to group together the hits of both the muon and electron. First, the charge hits on the wire plane of choice from the tagged muon-candidate track are looped over and the hit most closely matched in drift position to the X-coordinate of this track's starting point (where it enters the TPC) is designated as the clustering seed. Then, starting at the seed hit, all hits on the plane are looped over and the

next nearest hit (within a 2D distance $d<2.5 \mathrm{~cm}$, where $d=\sqrt{\Delta W^{2}+\Delta X^{2}}$ ) is added to the cluster. This is repeated until no new hits can be added to the cluster. This clustering process is then repeated starting again from the seed hit, but this time excluding hits that were clustered together in the first round. If hits can be added to this new cluster, then it implies the original clustering procedure was incomplete (this can happen if the true first 

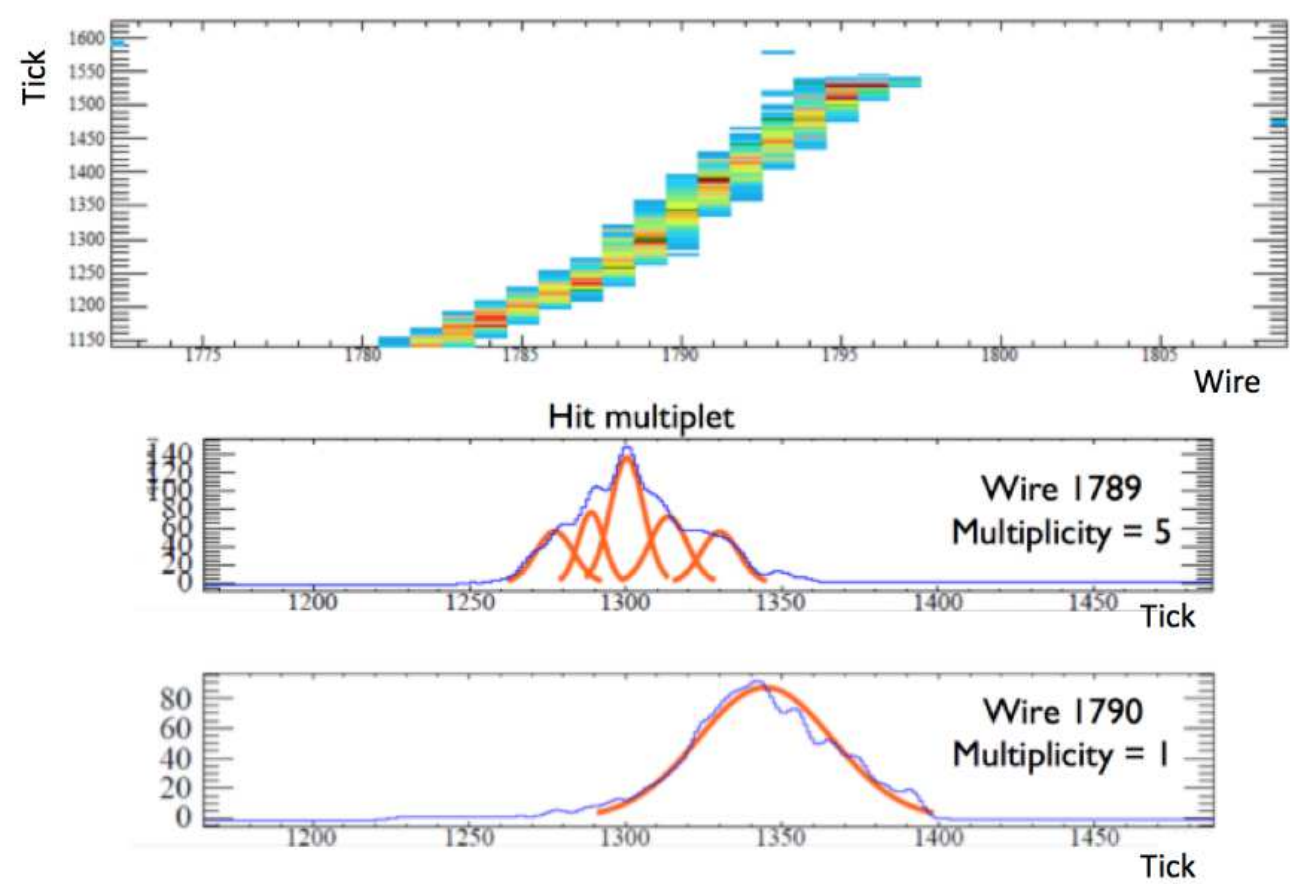

Figure 5.4: (top) Wire signals from a stopping track, and two examples of Gaussian hits fit to identified regions of interest (middle and bottom) [84].

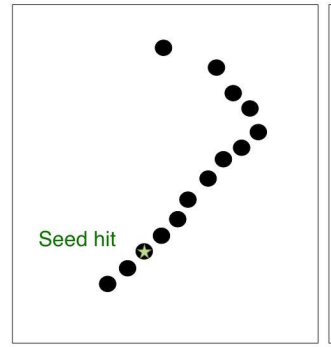

(1) Initial seed chosen

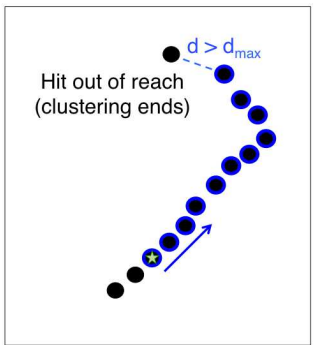

(2) $1^{\text {st }}$ pass clustering

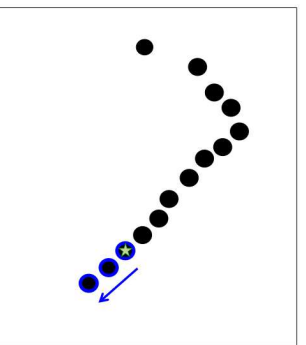

(3) $2^{\text {nd }}$ pass clustering

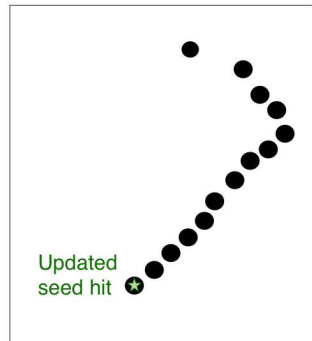

(4) Seed hit updated

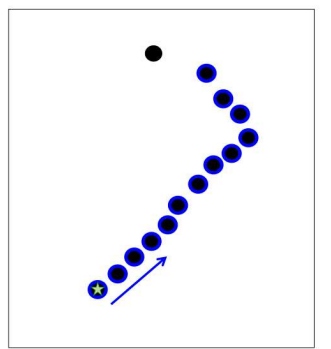

(5) Final cluster formed

Figure 5.5: Cartoon illustrating the proximity-based clustering process. Charge hits are represented as black circles in a 2D space. Clustered hits are shown with a blue outline. The seed hit is identified by a green star. 
hit from the muon track is not properly tagged as the seed due to incomplete tracking), and the entire clustering process is repeated using the endpoint of the second cluster as the new seed. This clustering procedure is depicted in a cartoon in Figure 5.5.

We now employ a technique developed by MicroBooNE in their analysis of Michel electrons [34], with several modifications to account for LArIAT's smaller active volume. Each hit in the cluster is assigned a distance value $(s)$ corresponding to the projected $2 \mathrm{D}$ distance of that hit along the cluster's trajectory (in W-X coordinate space). A smoothed-out profile of charge $(q)$ of each hit is constructed by calculating the local truncated mean in a neighborhood surrounding each hit along the cluster trajectory, truncating some fraction of both the highest- and lowest-charge points to minimize fluctuations from outliers. An example of a charge profile for a cluster in data is shown in the middle plot of Figure 5.6. with the truncated mean hit charge represented by the blue line while the dotted black line traces the charge of each individual hit.. The blue points connected by a solid blue line show the truncated mean along the cluster, while the dotted black lines trace the charge for each individual hit.

A profile of the local linearity (sometimes called the covariance) is also computed. The linearity $\chi_{i}^{2}$ at hit $i$ within a cluster is computed as:

$$
\chi_{i}^{2}=\frac{1}{N \sigma_{x} \sigma_{w}}\left|\sum_{i-b}^{i+b}\left[\left(X_{i}-\bar{X}\right) \times\left(W_{i}-\bar{W}\right)\right]\right|
$$

where $b$ is the number of hits defining the local neighborhood window, $N=2 b+1$ is the total number of hits in the neighborhood, and $\sigma_{x}$ and $\sigma_{w}$ are the standard deviations of the $\mathrm{X}$ and $\mathrm{W}$ coordinates, defined generically as

$$
\sigma_{v}=\sqrt{\frac{\sum_{i-b}^{i+b}\left(V_{i}-\bar{V}\right)^{2}}{N}}
$$

The mean values used are always the mean within the defined neighborhood, $\bar{V}=\sum_{i-b}^{i+b} V / N$. 
An example linearity profile is drawn in the bottom plot of Figure 5.6.

Using these two profiles, the boundary point between the muon and electron portions of the cluster is determined. A procedure is followed exploiting the muon's Bragg peak together with the kink formed by the decay electron:

1. The maximum in the truncated mean charge, $\langle q\rangle_{t}$, is first located. To avoid edge effects in the truncation process where outlier hits at the edges are not properly truncated, the first and last two points in the cluster are excluded. The hit with the maximal $q$ within a region following (and including) this point is identified as the candidate boundary point.

2. If the local linearity at this boundary hit or at least one of its adjacent hits is below some threshold, $\chi_{\text {thresh }}^{2}$, then this is declared the boundary hit. This ensures the hit occurs at or near a "kink" in the cluster likely corresponding to the start of the decay electron. The threshold of maximum allowable linearity at the identified boundary (or adjacent samples) is nominally $\chi_{\max }^{2}=0.8$.

If a boundary is identified, the cluster is divided up into a muon-track segment and an electron-track segment. In order to define a terminal muon direction vector in $2 \mathrm{D} \mathrm{W}-\mathrm{X}$ space, a line is fit to all muon's hits near the end of the track (separated by less than 20 hits from the end point) with local linearity above some threshold, $\chi^{2}>0.8$.

\subsubsection{Michel electron shower formation in $2 D$ and $3 D$}

A vector is fit to hits in regions of high local linearity at the end of the muon track (using a maximum of 20 hits) to determine the terminal direction of the muon, $\overrightarrow{d_{\mu}}$. A second vector corresponding to the outgoing electron, $\vec{d}_{e}$, is then drawn from the muon endpoint to the charge-weighted mean (in W-X space) of the electron-tagged hits. The $2 \mathrm{D}$ decay angle $\left(\theta_{2 D}\right)$

is defined as the angle between $\vec{d}_{\mu}$ and $\overrightarrow{d_{e}}$. All hits included in the electron-tagged portion of the cluster, in addition to all other previously-unclustered hits falling within a 2D "cone" 

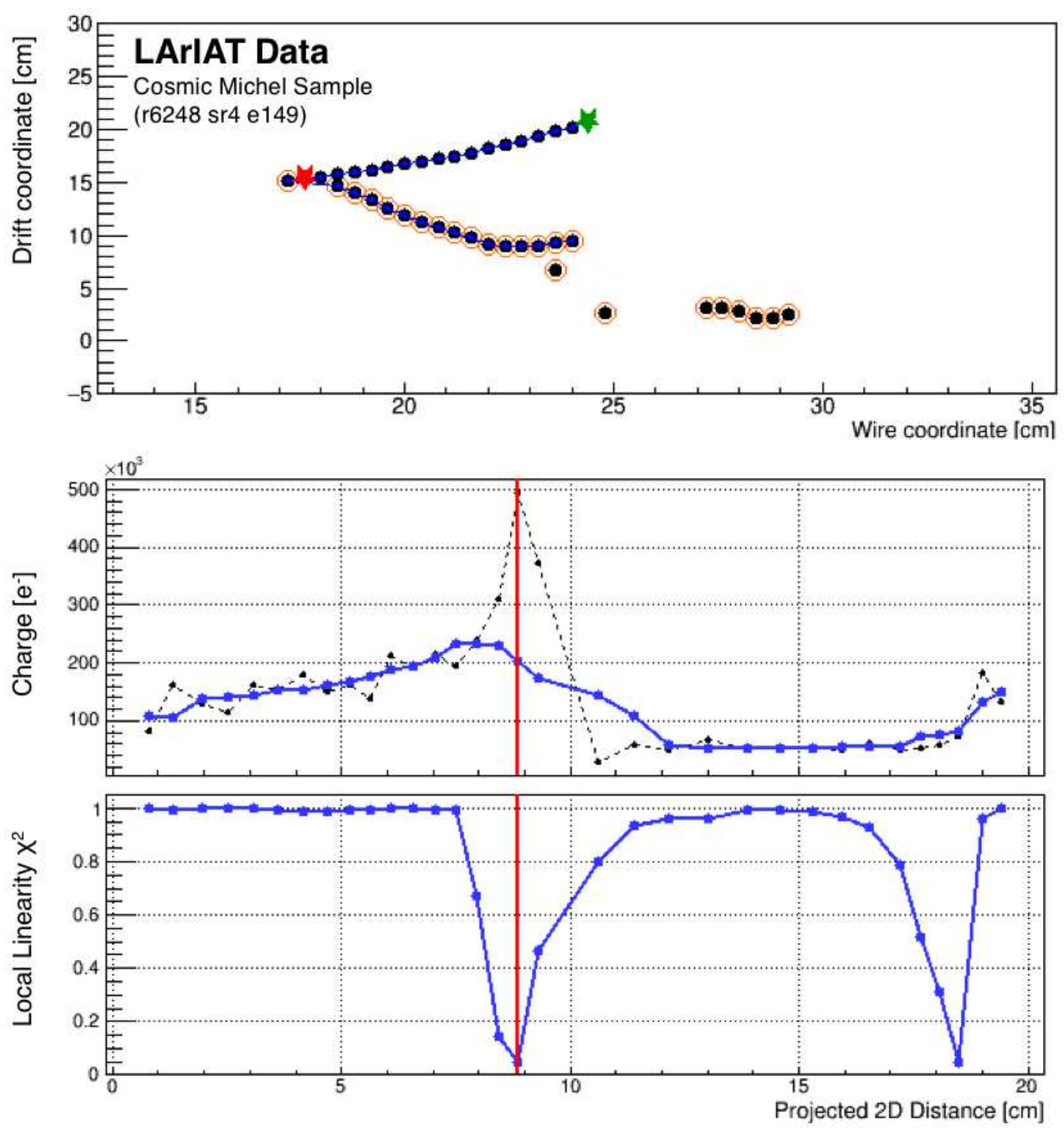

Figure 5.6: An example representation of the 2D Michel clustering procedure on the collection plane. In the top plot, wire hits are represented as black circles, and the smaller blue dots indicate hits that were included in the original combined $\mu+\mathrm{e}$ cluster. The green star marks the seed hit, while the red star marks the identified muon endpoint based on hit charge and linearity conditions described in the text. Hits included in the final Michel electron shower are circled in orange. 

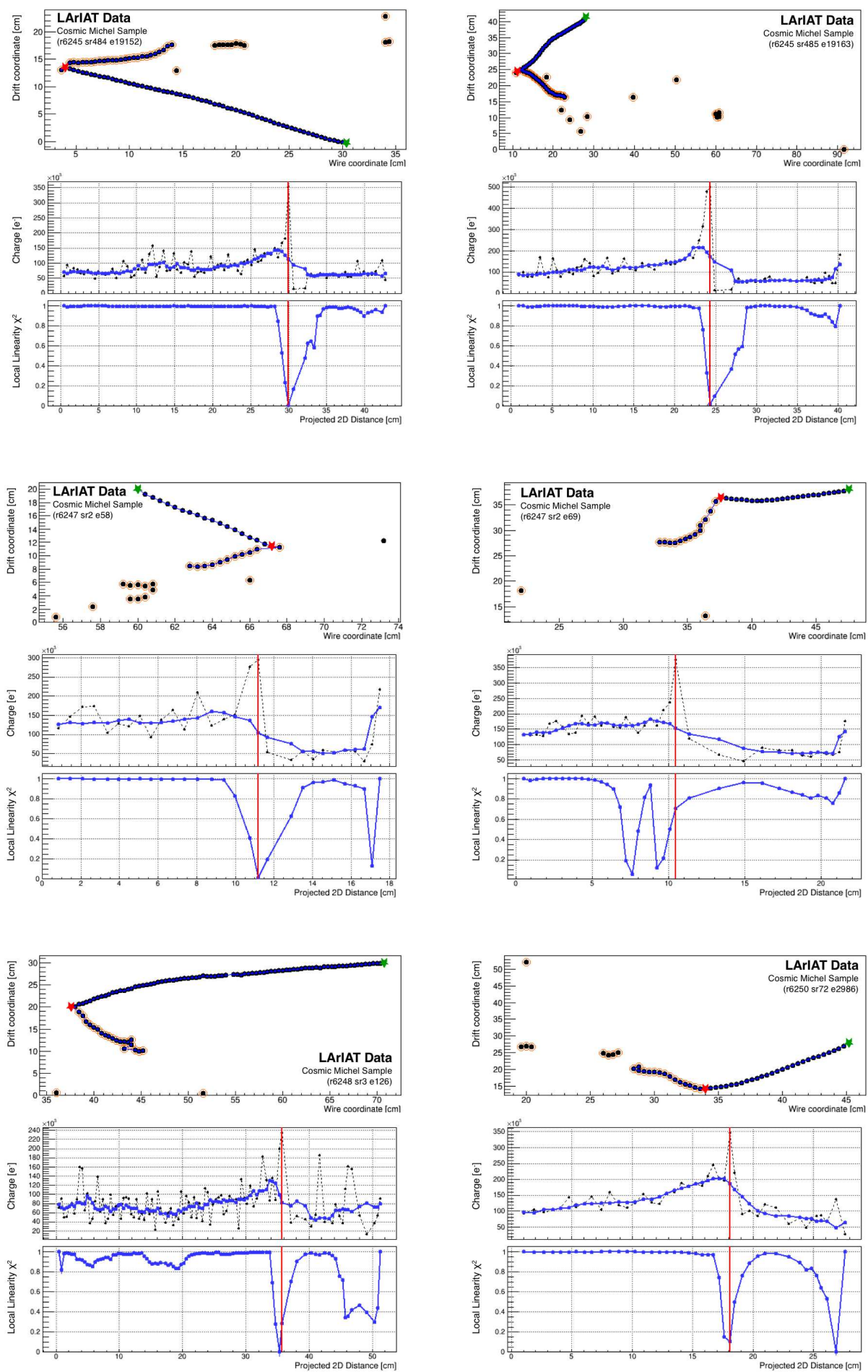

Figure 5.7: Clustering examples on the collection plane. See Figure 5.6 for an explanation of symbols. Note that charge has been expressed in units of electrons $\left(e^{-}\right)$; the ADC-to-electron conversion factor is introduced in Chapter 6 . 

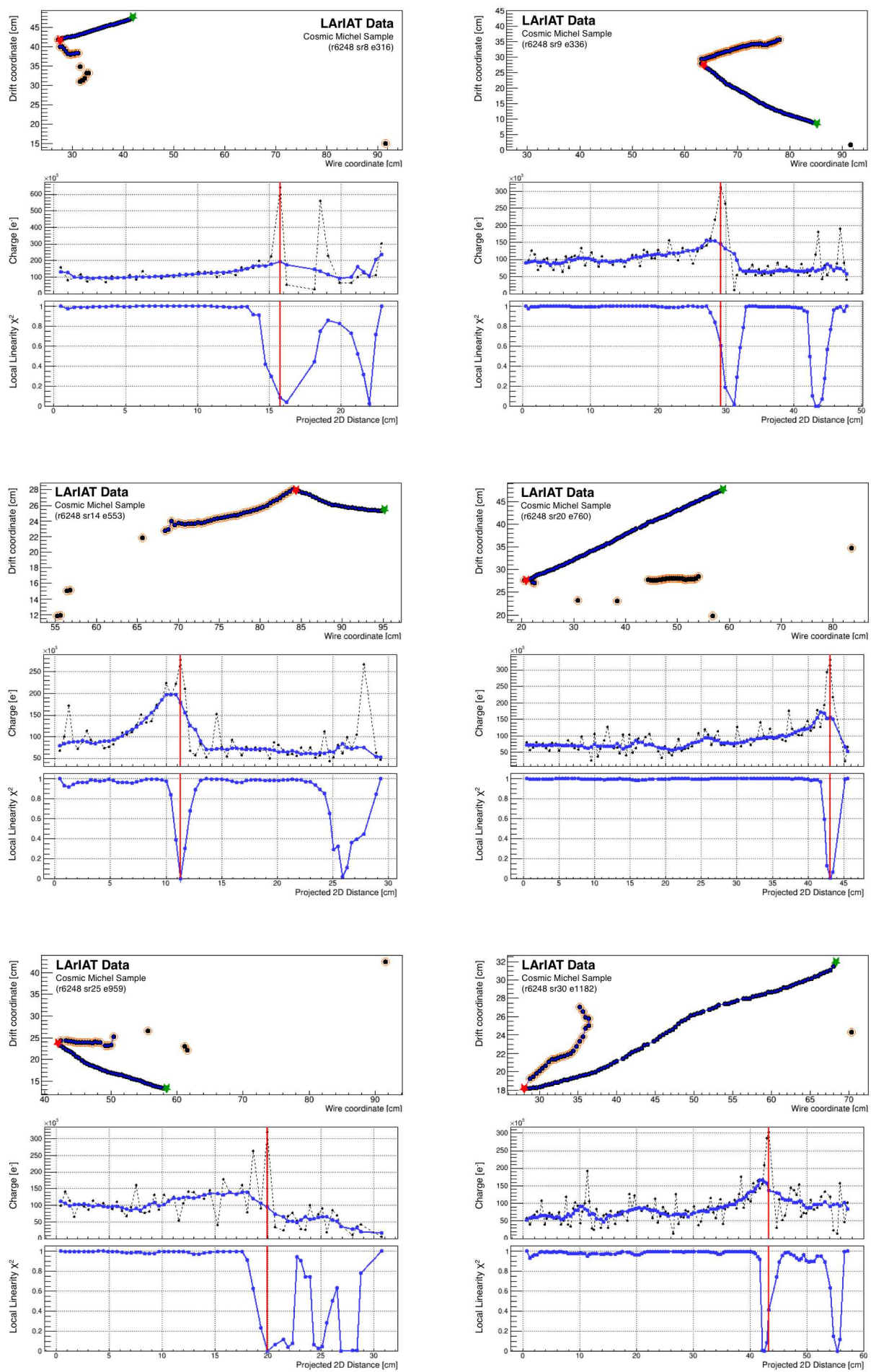

Figure 5.8: Clustering examples on the collection plane. See Figure 5.6 for an explanation of symbols. Note that charge has been expressed in units of electrons $\left(e^{-}\right)$; the ADC-to-electron conversion factor is introduced in Chapter 6 . 


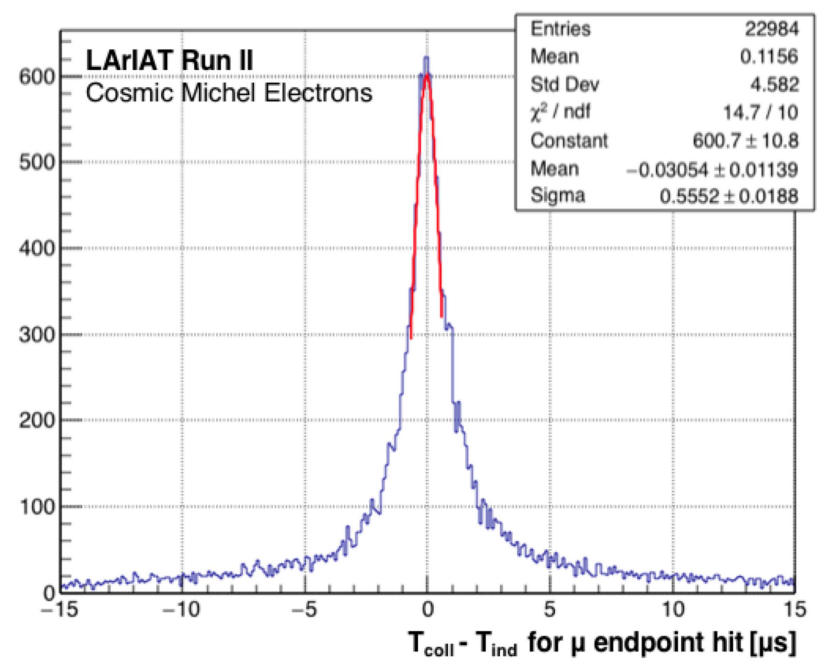

Figure 5.9: The difference in hit times between the collection and induction planes for hits identified as the muon endpoint in events where a muon-electron boundary was identified on both planes. The peak is fit to a Gaussian (red line).

with opening angle $\delta \theta=30^{\circ}$ directed along $\vec{d}_{e}$, are grouped into the Michel electron shower. In Fig. 5.6, hits belonging to the electron shower are outlined in orange. In principle, this will include both the direct ionization from the electron as well as any displaced charge deposited by bremsstrahlung photons emitted by the electron in the EM showering process [87]

The 2D clustering and shower reconstruction steps discussed up to now are repeated on both the collection and induction wire planes. We attempt to form 3D space points within the shower by combining information from both wire planes. If a shower is successfully reconstructed on both planes, inter-plane hits separated in time by less than some threshold, defined by their respective hit widths $\sigma_{h}\left(\Delta<\sqrt{\left.\left(\sigma_{h}^{\text {ind. }}\right)^{2}+\left(\sigma_{h}^{\text {coll. }}\right)^{2}\right)}\right)$, are paired up starting with those that are most closely-matched in time. To determine if there is a timing offset between the planes that hasn't been corrected, we plot a distribution of hit time differences between hits identified as the muon endpoint on each plane, shown in Figure 5.9. A Gaussian fit to the peak of this distribution suggests an offset of only $-0.03 \pm 0.01 \mu$ s, indicating good inter-plane time alignment.

For each pair of matched hits, a $Y$ and $Z$ coordinate is calculated from the intersection 
of the two wires. Together with the drift coordinate $X$, a full 3D space point is thus formed.

\subsection{Optical reconstruction}

The reconstruction of signals from the two cryogenic PMTs is now addressed. This process involves several steps necessary to remove noise and overshoot, followed by gradient-based optical pulse-finding. The identification of the characteristic Michel electron topology, defined by two pulses delayed by $\sim 2.2 \mu \mathrm{s}$, is described.

\subsubsection{Waveform cleanup}

PMT waveforms are first truncated from their full size of 28672 samples down to the first 18000 samples for faster processing. In order to minimize electronic fluctuations that could fake hits in the signal gradient scan to follow, the waveform is "smoothed" in that each sample is replaced by the average of that sample and the two adjacent. There is a period of $8.4 \mu \mathrm{s}$ (a "front porch" or pedestal region) preceding the sample corresponding to the trigger time in each waveform, and the Michel coincidence gate plus its delay extend to a maximum of about $7.3 \mu$ s prior to this trigger time. Therefore, the waveform pedestal and its RMS is calculated using the first $1 \mu \mathrm{s}$ of the waveform, since this region is unlikely to include activity related to the event. This pedestal is subtracted from the full waveform.

\subsubsection{PMT overshoot correction}

An overshoot was observed in signals from the larger HMM PMT in the Run IIB dataset. To prevent a systematic mis-measurement of pulse areas, we craft a correction for this overshoot by exploiting an observed linear relationship between the integral of the peak and the amplitude of the overshoot. Inverted average HMM waveforms were compiled for nine ranges of pulse amplitudes and each waveform was fitted from $9 \mu$ s to $18 \mu$ s to the function $f(t)=-A e^{-t / \tau_{r}}$. The baseline recovery lifetimes $\tau_{r}$ from all fits were averaged together to

determine the average lifetime $\tau_{r}^{a v e}=11.5 \mu \mathrm{s}$. The fits were repeated with $\tau_{r}$ fixed to $\tau_{r}^{a v e}$ 


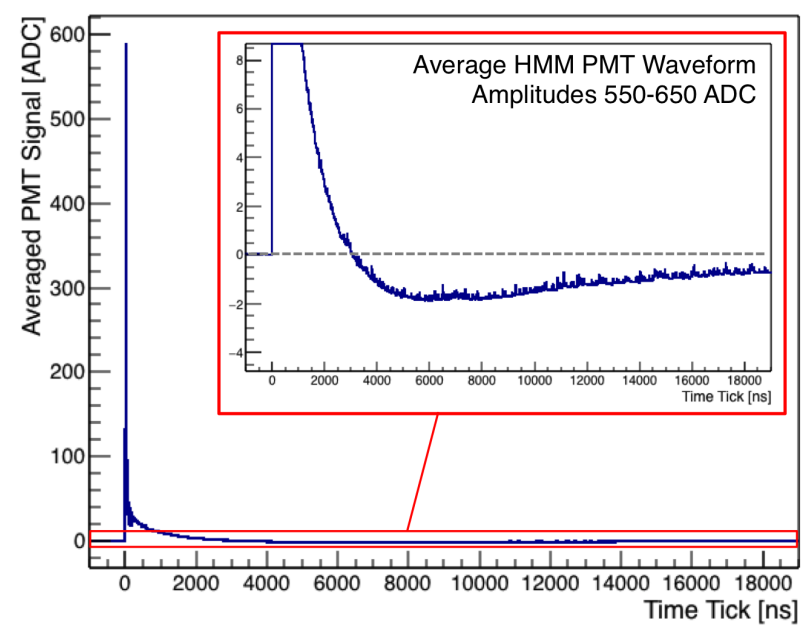

Figure 5.10: Overshoot in the Hamamatsu (HMM) PMT average waveform from Run IIB.

and the normalization parameter $A$ was recorded for each. A linear relationship shown in Figure 5.12 was found between this overshoot normalization and the integral $\left(I_{50}\right)$ of the pulse peak, integrated in the range $t=[-5,45] \mathrm{ns}$,

$$
A\left(I_{50}\right)=0.2777+0.0002466 \times I_{50}
$$

To apply this correction during reconstruction of optical data, each pulse in the waveform (found using the hit-finding methods to be described later) is first identified. For each found pulse $i$, a baseline correction of

$$
f_{\mathrm{corr}}^{\mathrm{BS}}(t)= \begin{cases}0, & t<t_{i} \\ -A \cdot \exp \left(\frac{-\left(t-t_{i}\right)}{\tau_{r}^{\text {que }}}\right), & t \geq t_{i}\end{cases}
$$

is subtracted from all samples, where $t_{i}$ is the time-zero of the specific pulse. Since this is repeated for each pulse, the total baseline correction is cumulative across the waveform.

\subsubsection{Adaptive running baseline subtraction}

To remove slow oscillations in the PMT signal $s(t)$, a modified running baseline is com- 

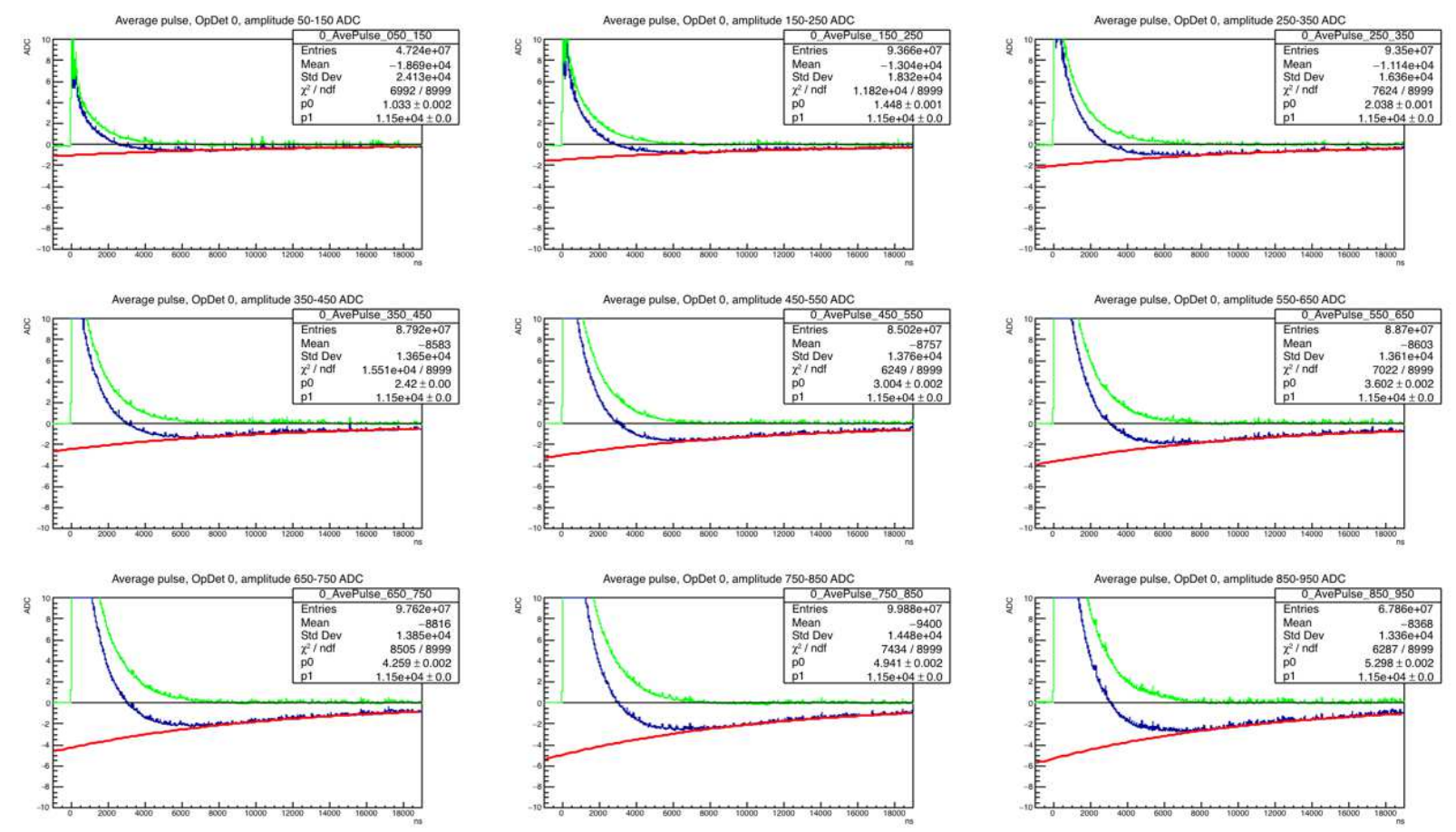

Figure 5.11: Individual exponential fits to the overshoot in average HMM PMT waveforms for all 9 amplitude ranges. The blue line shows the original averaged waveform, and the green line shows the waveform after the overshoot correction [87].

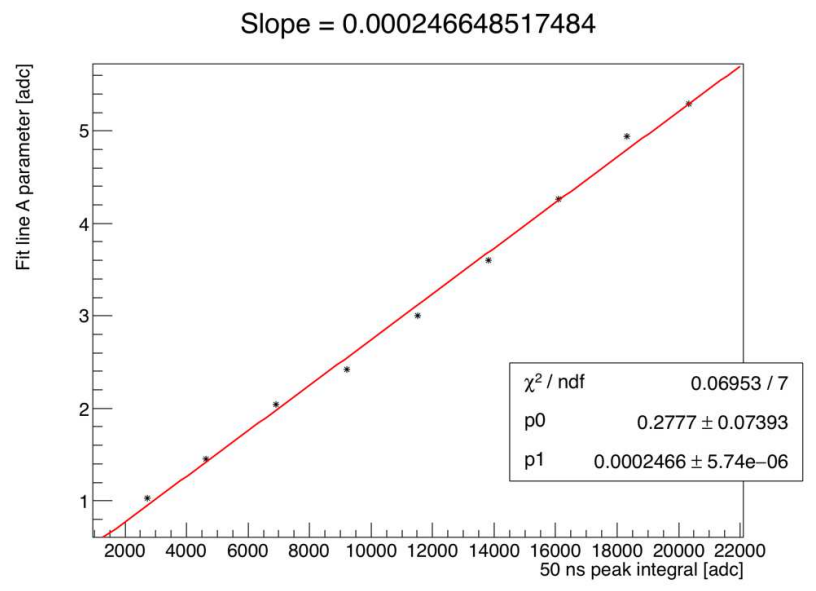

Figure 5.12: Linear fit of $50 \mathrm{~ns}$ pulse integrals (integrated between $t=-5 \mathrm{~ns}$ and $t=45 \mathrm{~ns}$ ) to the undershoot normalization $A$ extracted from each fit [87]. 

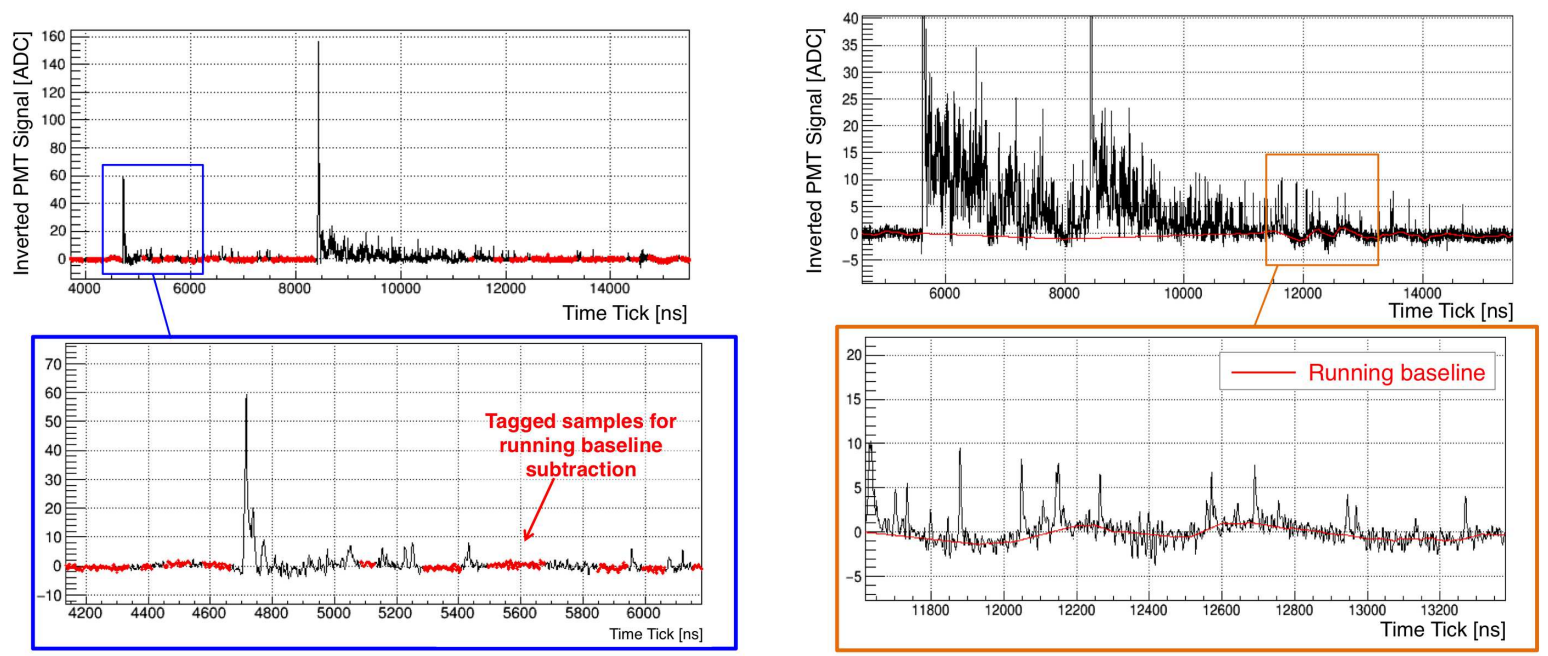

Figure 5.13: Tagged regions of a sample waveform to be used in the calculation of the running baseline (left), and the resulting running baseline for an example waveform shown (right).

puted with a gradient-based technique which masks out regions where activity is detected. This process is illustrated in Figure 5.13. First the signal gradient $g(t)$ is computed using a formula that replicates a 5th-order derivative,

$$
g\left(t_{i}\right)=\frac{1}{12}\left[f\left(t_{i-2}\right)-8 f\left(t_{i-1}\right)+8 f\left(t_{i+1}\right)-f\left(t_{i+2}\right)\right]
$$

The RMS of the gradient, $\sigma_{g}$, is calculated over a baseline region $1 \mu$ s in length. Samples with $s(t)>5 \mathrm{ADC}$ or $g(t)>3 \sigma_{g}$ are marked as the start of regions containing activity, and the following 50 samples are designated as "active." A standard running baseline is computed over the inactive or "quiet" regions using a truncated mean taken from quiet regions that lie within 50 clock ticks of the current sample. A linear extrapolation extends this running baseline across tagged active regions, connecting the first sample of each quiet region to the last sample of the preceding quiet region. This running baseline is then subtracted from the waveform [87].

\subsubsection{Optical hit-finding}

To locate hit candidates, the gradient using Equation 5.7 is computed at each sample 
in a PMT waveform and samples with gradient values higher than a specified threshold are tagged as hit candidates. The RMS of the gradient in a region preceding the hit (ranging from $-100 \mathrm{~ns}$ to $-5 \mathrm{~ns}$ ) is calculated and the hit is eliminated if the gradient's local maximum in the neighborhood of the hit candidate sample fails to exceed a multiplicative factor $(\times 3)$ of the RMS. Hit candidates separated by less than a specified minimum distance from the preceding hit are eliminated.

\subsubsection{Hit reconstruction and muon decay time measurement}

The goal of optical hit processing is to determine properties like pulse amplitude, inte$\operatorname{gral}(\mathrm{s})$, and width for each of the identified hits. The signal gradient $g(t)$ is thresholded to identify optical hits. If there are exactly two hits found, the muon decay time $\Delta T$ is measured as the difference between the time of the first (muon candidate) and second (Michel electron candidate) pulse. An integral of the first $100 \mathrm{~ns}$ of the electron candidate pulse determines the prompt light $S_{100}$; an integral over $7 \mu$ s determines the total light $S$.

\subsubsection{Muon late-light subtraction}

Light produced by the stopping muon will overlap with the Michel candidate integration window to varying degrees depending on the decay time $\Delta T$. For events with a large enough time separation between the muon and electron pulses, we attempt to correct for this muon late-light contamination by fitting the region of the muon's late light between 400-1800 ns and extrapolating the fit forward.

First, the late-light signal following the muon pulse is binned into six regions with varying widths ranging between 100 ns and 300 ns (400-500 ns, 500-700 ns, 700-900 ns, 900-1200 ns, 1200-1500 ns,1500-1800 ns). The center of each bin is treated as the $t$-coordinate and the signal density (ADC/ns) per bin is calculated by dividing each bin content by its width. A fit is performed to a function of form:

$$
S_{\text {late }}(t)=A e^{-t / \tau_{t}^{\prime}}+B e^{-t / \tau^{*}}
$$



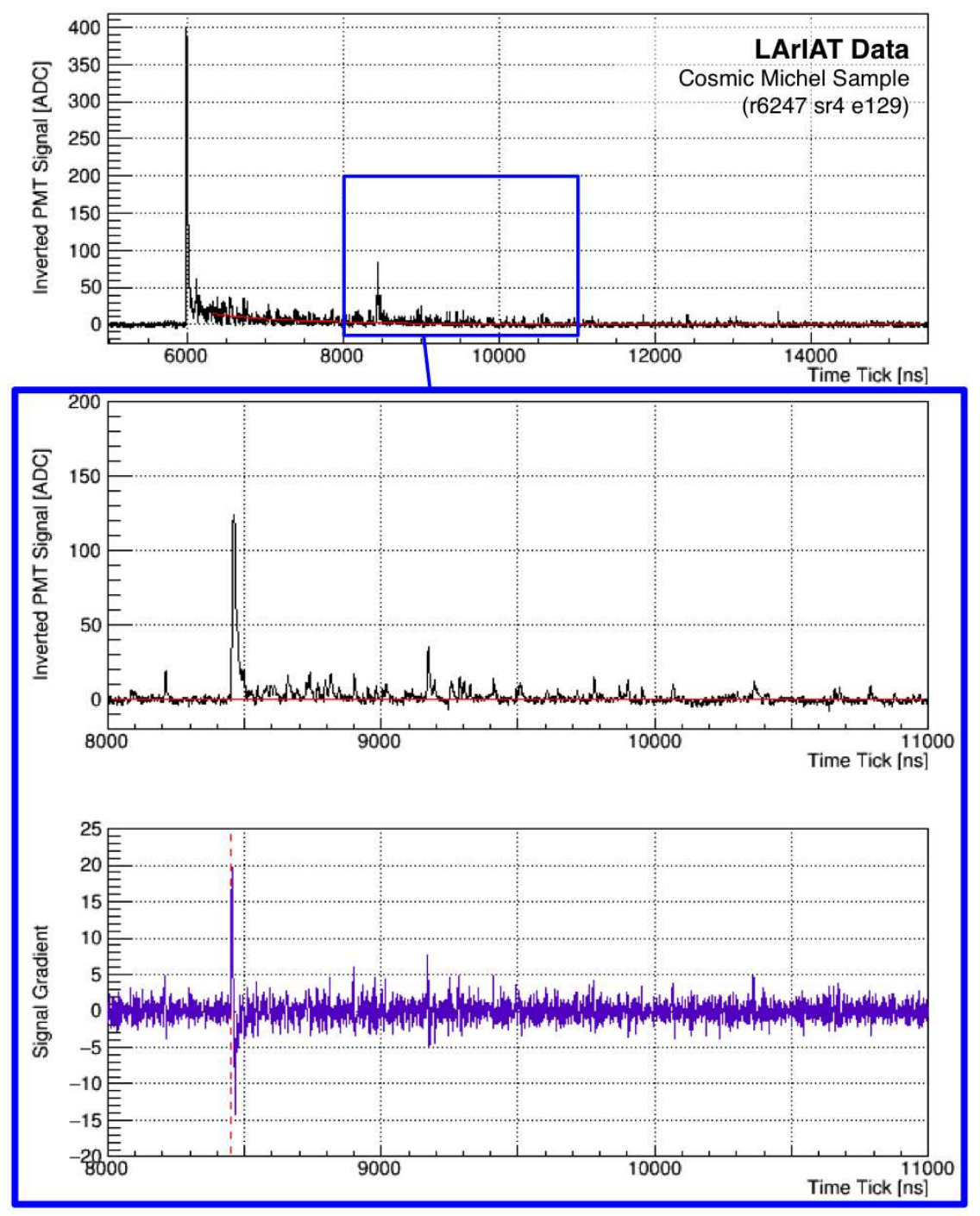

Figure 5.14: An inverted ETL PMT waveform with a zoomed-in view of the Michel candidate pulse for an event with decay time $\Delta T \approx 2.5 \mu \mathrm{s}$. The violet trace in the bottom plot is the signal gradient, with a dotted-red line signifying the identified location of the hit. The solid red line overlaid on the PMT signal plot is the estimated (fitted) muon late-light component which is subtracted off in the integration of the Michel pulse. 
where $A$ is treated as a free parameter and $\tau_{t}^{\prime}$ is taken as the effective quenched triplet-state excimer lifetime inferred from fits to average muon waveforms in the range of $0.4-2 \mu \mathrm{s}$ (see Appendix A). The second term in $S_{\text {late }}(t)$ is meant to account for contamination from the component of TPB fluorescence with the longest lifetime, $\tau^{*}=3.55 \mu \mathrm{s}$ [67], since this extra contribution is not effectively handled by a simple single-exponential fit. The normalization factor $B$ is fixed to values based on fits of Equation 5.8 to average waveforms from an independent sample of cosmic muons that cross the TPC without stopping. See Figure 5.15 for a more detailed description of this procedure. ${ }^{1}$

This fitted function $S_{\text {late }}(t)$ is extrapolated through the integration regions of the Michel pulse to get an estimation of the contamination, which is then subtracted off the raw integrals accordingly. Corrected integrals for the "total light" $S(7-\mu$ s integral) and the "prompt light" $S_{100}(100-n s$ integral) for the Michel electron are saved.

\subsection{Muon decay time spectra and measurement of capture lifetime}

Here we present the resulting muon decay time spectra from our two datasets, using the results to validate the sample and estimate its purity. In order to enhance statistics and minimize optical hit-finding effects that manifest at small $\Delta T$, we use a slightly different event selection for measuring the muon decay time spectrum. Since there is no intention to use any calorimetric information from the wires here, the only event selection requirement is that a stopping 3D track was identified with an optical topology consistent with a delayed Michel electron decay. (The result when also requiring a $2 \mathrm{D}$ shower on the collection plane can be seen in Table 5.2.)

When plotting $\Delta T$ against optical hit characteristics such as hit amplitude, we notice anomalies at low- $\Delta T$ (see Figure 5.16). This is especially prominent in Run IIB, with two distinct populations of low-amplitude events contaminating very specific values of $\Delta T$. It's

1. It's unclear why there is a data-MC discrepancy in the ratios of the slow TPB component to the fitted LAr scintillation component. Possible causes are waveform cleanup and smoothing techniques which may be inadvertently suppressing photons in the tails. 

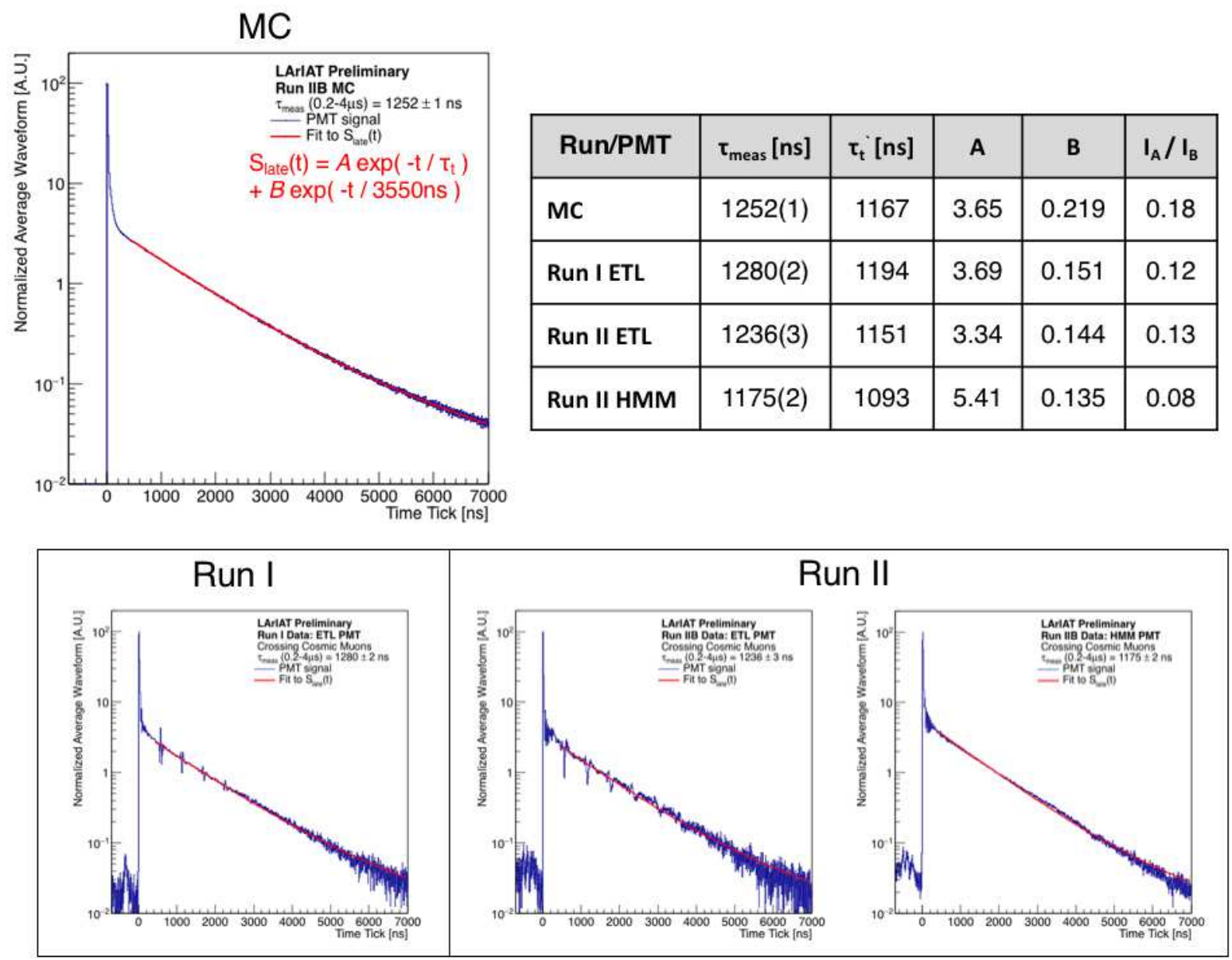

Figure 5.15: Fits to the average waveforms of crossing muon events. First the region 0.4$2 \mu \mathrm{s}$ is fit to a single exponential to extract $\tau_{\text {meas }}$. Then, Equation A.1 is used to estimate the true quenched triplet lifetime $\tau_{t}^{\prime}$. A fit to $S_{\text {late }}(t)$ from Equation 5.8 is then performed over a larger time range, 0.4-7 $\mu$ s, with parameter $\tau_{t}^{\prime}$ fixed. The ratio of the integral of the second component of the fit to the integral of the first component is computed and used to constrain the normalization parameter $B$ in fits during the late-light subtraction procedure in reconstruction: $B=f A \tau_{t}^{\prime} / 3550$, where $f=I_{A} / I_{B}$. 
LArIAT Run I

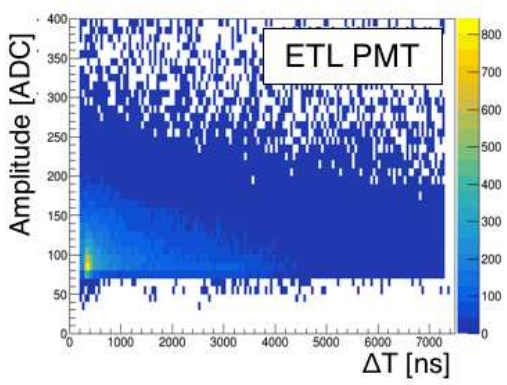

LArIAT Run IIB

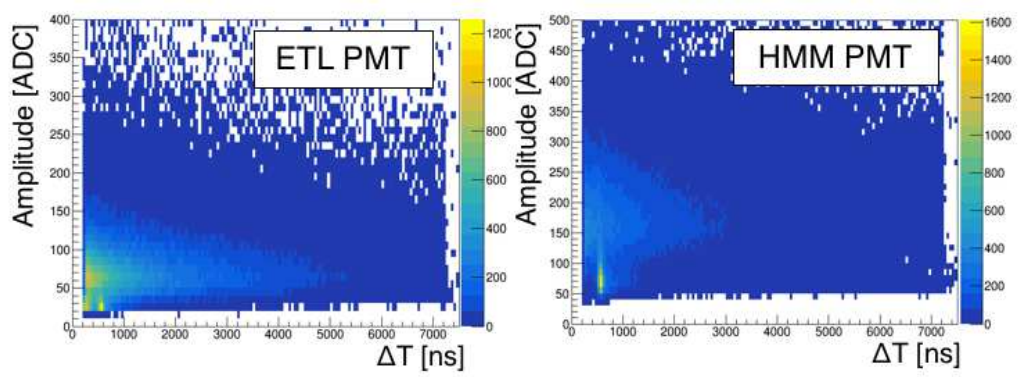

Figure 5.16: Amplitude of Michel-candidate optical pulses plotted against the measured decay time for the Run I (left) and Run IIB (right) datasets. For Run I, events are accepted with amplitude $>80$ ADC (on the ETL PMT). For Run IIB, we similarly impose an amplitude cut of 130 ADC for the HMM PMT and 45 ADC for the ETL PMT.

unclear what could be causing this (afterpulsing, reflections, noise hits, etc..). We place cuts on pulse amplitude to exclude these anomalous events which would otherwise distort our decay time fits. We can safely place these cuts since decay time and and the energy of the Michel electron are independent of one another.

Resulting muon decay time spectra are shown in Figure 5.17. Each distribution of decay times is fit to a double exponential function,

$$
f(t)=\left[C_{\mu^{+}} \times e^{-t / \tau_{\mu^{+}}}\right]+\left[C_{\mu^{-}} \times e^{-t / \tau_{\mu^{-}}}\right]+B,
$$

where $B$ is a constant background term, $\tau_{\mu^{+}}$is the positive muon lifetime of $2197 \mathrm{~ns}$ [79], and $\tau_{\mu^{-}}$is the effective lifetime of negative muons decaying in orbit from muonic Ar.

In Run I, the measured effective negative muon decay lifetime $\left(\tau_{\mu-}=504 \pm 40 \mathrm{~ns}\right)$ is significantly lower than the expected theory value of $615 \mathrm{~ns}$. This is likely due to only one PMT being used during this Run. Without a second PMT to cross-check and verify true scintillation pulses, this decay time spectra is likely to be contaminated with events at low- $\Delta T$ due to accidental noise hits being found within the late-light of the muon.

From the Run IIB dataset, we find $\tau_{\mu^{-}}=626 \pm 48 \mathrm{~ns}$, which suggests a muon capture 

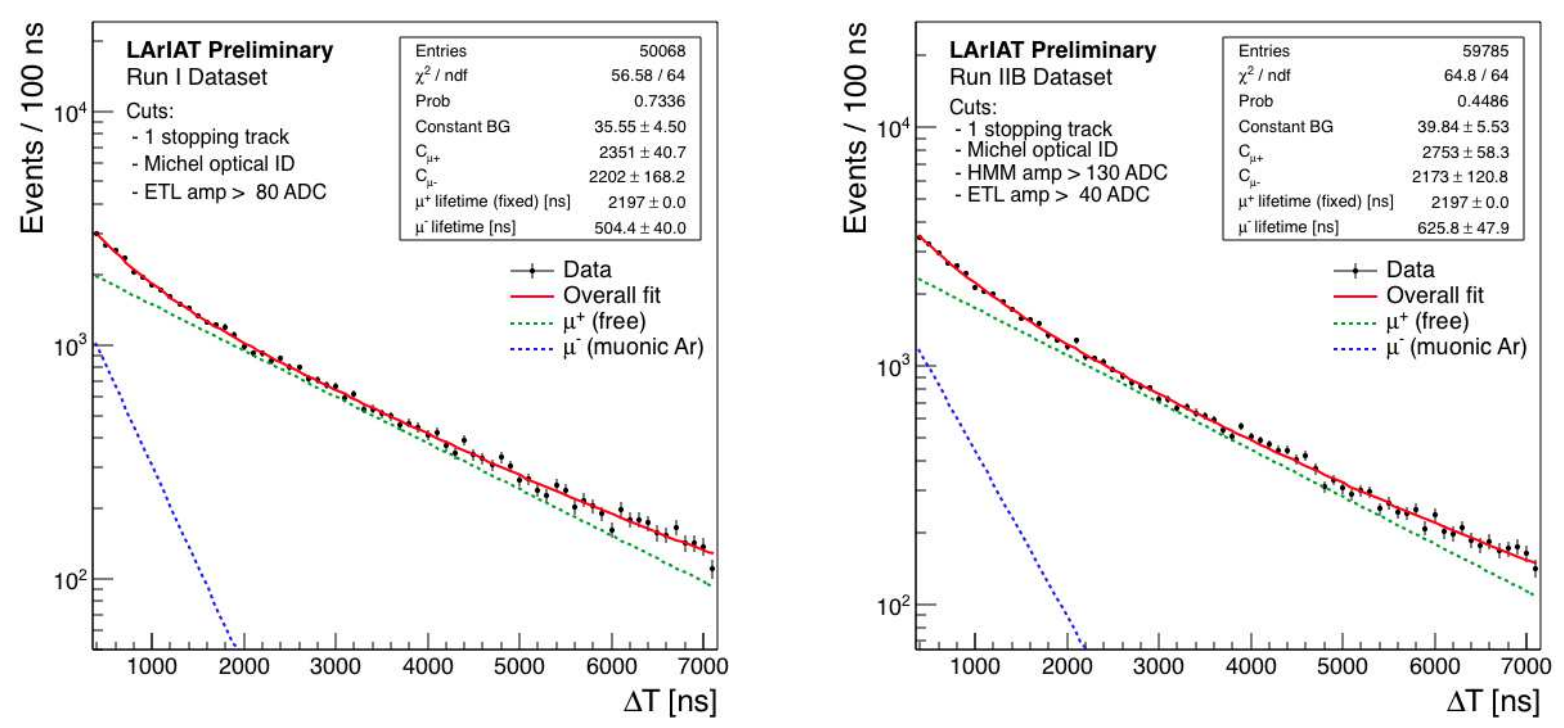

Figure 5.17: Muon decay time data for Run I (left) and Run IIB (right) datasets. In Run I, only one PMT was used. Without a second PMT to verify true scintillation pulses, the Run I data is more likely to contain accidental noise events that will warp our spectra.

lifetime of

$$
\tau_{c}=871 \pm 93 \mathrm{~ns},
$$

in agreement with the theoretical expectation and previous measurements [80, 81]. The corresponding capture probability from our data is

$$
P_{c}=\frac{k_{c}}{k_{\text {total }}}=\left(\frac{\tau_{c}}{\tau_{\mu^{-}}}\right)^{-1}=71.8 \pm 2.2 \% .
$$

The flat background term, $B=40 \pm 6$ events/bin, comprises $\approx 2740$ events integrated across the relevant decay times. We therefore estimate the contamination from non-Michel events to be $<5 \%$. Additional event quality cuts are expected to improve upon this purity in the final selected sample used for calorimetric studies presented in Chapter 8 .

We can use the relative normalization of the $\mu^{+}$and $\mu^{-}$populations to estimate the cosmic muon charge-ratio in our sample,

$$
\frac{\mu^{+}}{\mu^{-}}=\frac{N_{\mu^{+}}}{N_{\mu^{-}}}=\frac{C_{\mu^{+}} \tau_{\mu^{+}}}{C_{\mu^{-}} \tau_{\mu^{-}}\left(1-P_{c}\right)^{-1}}
$$


Table 5.2: Summary of results for the negative muon lifetime.

\begin{tabular}{|l|c|c|}
\hline Selection & Run I & Run IIB \\
\hline 1 stopping trk + optical ID & $504(40) \mathrm{ns}$ & $626(48) \mathrm{ns}$ \\
\hline 1 stopping trk + optical ID + 2D shower & $511(56) \mathrm{ns}$ & $662(62) \mathrm{ns}$ \\
\hline
\end{tabular}

where $C_{\mu^{+}}$and $C_{\mu^{-}}$are the normalization factors for the two exponential decay components in the fit. The result,

$$
\frac{\mu^{+}}{\mu^{-}}=1.27 \pm 0.16
$$

is consistent with a previous measurement of $\mu^{+} / \mu^{-}=1.25$ from CMS [93], which used a sample of muons in the momentum range of $5 \mathrm{GeV} / \mathrm{c}$ to $1 \mathrm{TeV} / \mathrm{c}$. Our sample probes the lowest-energy tail of the distribution since the muons must be low enough in energy to stop in the 40-cm-tall LAr active volume of the LArIAT TPC.

The optical hit-finding procedure may have an efficiency that is a function of $\Delta T$, particularly for the Hamamatsu PMT due to its overshoot. Michel electron pulses that closely follow the $\mu$ pulse may suffer from a suppressed signal response as the PMT recovers and the dynodes return to their nominal voltage. A full and accurate simulation of the PMT electronics would need to be carried out to evaluate the impact of this effect on our measurement. For the time being, our cut at higher pulse amplitudes should mitigate this time-dependent effect. 


\section{6}

\section{Detector Calibrations}

Here we discuss the calibration factors needed to reverse detector effects (like drift attenuation and losses due to propagation) and convert raw signals, in units of ADCs, into physical quantities like electrons or photoelectrons (pe). Such corrections are an essential component in the calorimetric reconstruction presented in Chapter 8 .

\subsection{Charge-based calibrations}

First we describe the calibrations necessary for reconstructing charge, including (1) correcting for electron attenuation from impurity quenching; (2) addressing non-uniformities in the responsiveness of wires across the collection plane; and (3) extracting the electronics calibration factor needed to convert ADCs from integrated wire pulses into a quantity of collected electrons.

\subsubsection{Charge attenuation lifetime during drift}

As noted in Section 3.3.1, electrons in LAr will attach to residual electro-negative contaminants, leading to an attenuation factor of $C / C_{0}=\exp \left(-t / \tau_{e}\right)$ where $C_{0}$ and $C$ are free electron concentrations at the site of deposition and at the anode wires, respectively.

Samples of cosmic muons crossing the full diagonal of the TPC, triggered by a pair of scintillator paddles arranges outside the cryostat, are used to measure $\tau_{e}$ throughout the course of the experiment. Since the muons are passing through the TPC, we assume they are uniformly MIP-like in their energy deposition density throughout their trajectory. The full $\approx 320 \mu$ s drift time is divided into 10 bins and values of charge deposition density $d Q / d x$ measured along the tracks are added to separate histograms for each bin. Each histogram is fit to a convolution of a Landau (the shape of the true distribution of $d Q / d x$ expected) and a Gaussian (detector resolution/smearing effects). The most probable value (MPV) 


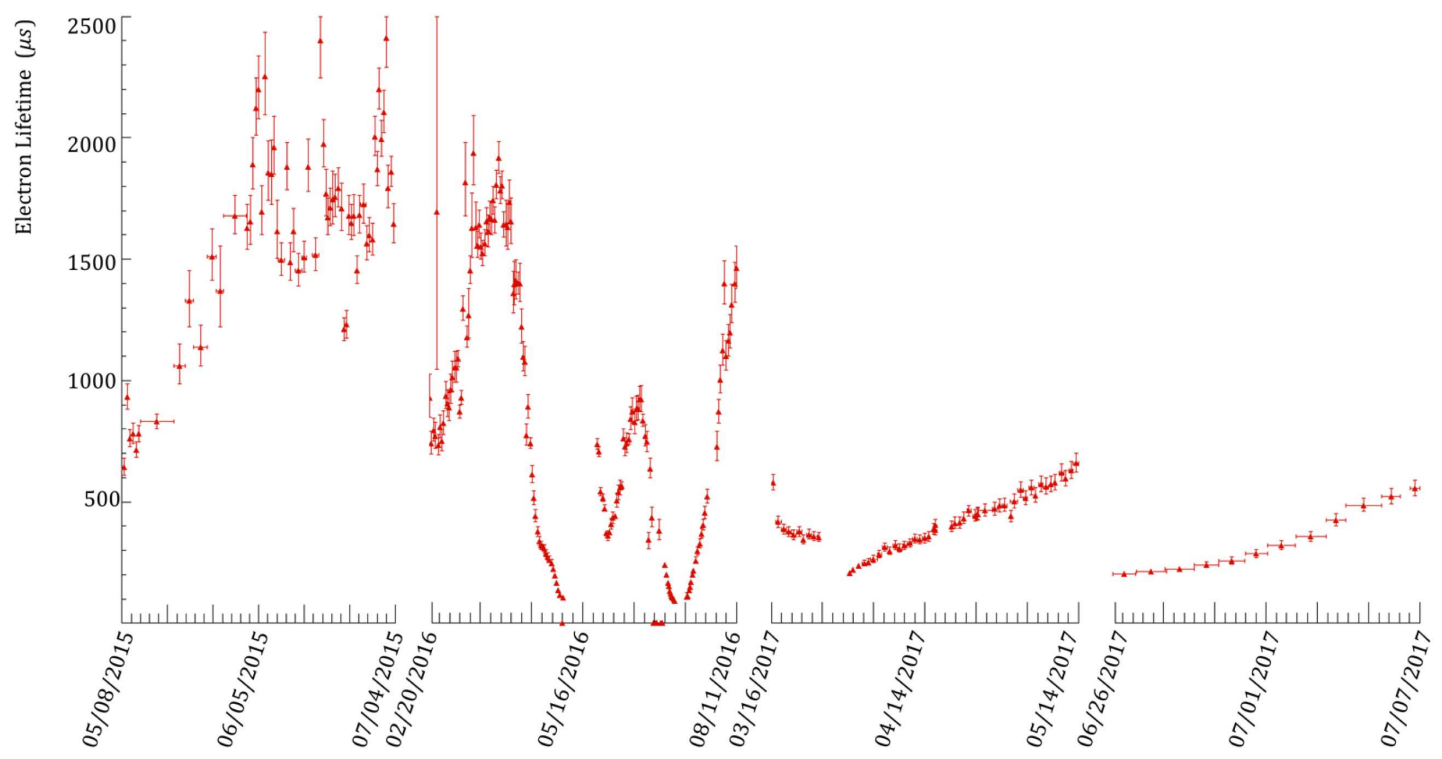

Figure 6.1: Electron lifetimes in LArIAT measured over different run periods.

from the Landau in each fit is plotted against the average drift time of the bin and a falling exponential is fit to extract $\tau_{e}$. This procedure is described in more detail in [69, 88]. The values of $\tau_{e}$ measured for LArIAT's different run periods are plotted in Figure 6.1. Visible in this plot are several periods of time during which the lifetime was observed to rapidly decrease due to unexpected sources of contamination.

\subsubsection{Wire-by-wire charge response uniformity}

Variations in the average response (i.e., signal amplitude) for lifetime-corrected MIP signals across the wire planes was observed. Specifically, the MPV of $d Q / d x$ distributions varied. A table of correction factors was compiled, the entry for each wire set to the mean MPV across the plane divided by the MPV observed for that wire. Raw signals from the wires are scaled by these correction factors on a wire-by-wire basis prior to any deconvolution or hit reconstruction [89].

\subsubsection{Charge response amplitude using stopping muons}

Understanding the charge response of wires in a LArTPC is essential for calorimetry. 
To calculate the number of drift electrons that were collected to produce a wire hit, we use an ADC-to-electron conversion constant which is a property of the read-out electronics and signal filtering process. These plane-specific calibration constants, $C_{e}^{\text {cal }}$, represent the average number of integrated ADC units for each detected electron and are used to reconstruct the charge $Q$ of each hit:

$$
Q\left[e^{-}\right]=\frac{\text { Hit area }[\mathrm{ADC}]}{C_{e}^{\mathrm{cal}}\left[\mathrm{ADC} / \mathrm{e}^{-}\right]}
$$

The charge response can be determined through charge injection studies in a dedicated electronics test-stand, or by looking at the energy deposition rate $(d E / d x)$ from known particles in data. As discussed in Chapter 3, the rate at which charged particles deposit energy depends on their mass and momentum, and the distribution of energy loss follows a skewed Landau for particles traversing some thickness of material (see Equation 3.3 and Figure 3.3). As muons in the Michel electron event sample slow down and lose momentum, they deposit an increasing amount of energy per unit length as predicted by Bethe-Bloch. This results in a well-known pattern of increasing charge deposition density along the track prior to the muon's stopping point, known as a Bragg peak, which can be fit from data to tune the charge response constant.

First we recontruct $d Q / d x$, in units of $\mathrm{ADC} / \mathrm{cm}$, along well-reconstructed stopping muon tracks identified in the cosmic Michel electron sample. This profile is then fit to the expected $d Q / d x$ in units of $\mathrm{e}^{-} / \mathrm{cm}$ calculated using Equation 3.3 and the Modified Box recombination model, with the calibration constants for converting ADC to electrons treated as free parameters. We describe the stages of this procedure in more detail below [69, 90]:

- Track selection: If a muon-electron boundary in the 2D Michel clustering from Chapter 5 is successfully identified on both the collection and induction planes, and if these hits align in time within a threshold defined by their respective hit widths $\sigma_{h}$ $\left(\delta t<\sqrt{\left.\left(\sigma_{h}^{\text {ind. }}\right)^{2}+\left(\sigma_{h}^{\text {coll. }}\right)^{2}\right)}\right)$, then they are used to form a 3D space point, $\mathbf{P}_{\text {bnd }}$. We require that the distance between $\mathbf{P}_{\text {bnd }}$ and the endpoint found from 3D-reconstructed track (Section 5.3) associated with the muon $\mathbf{P}_{\text {trk }}$, projected along the direction of 3D 

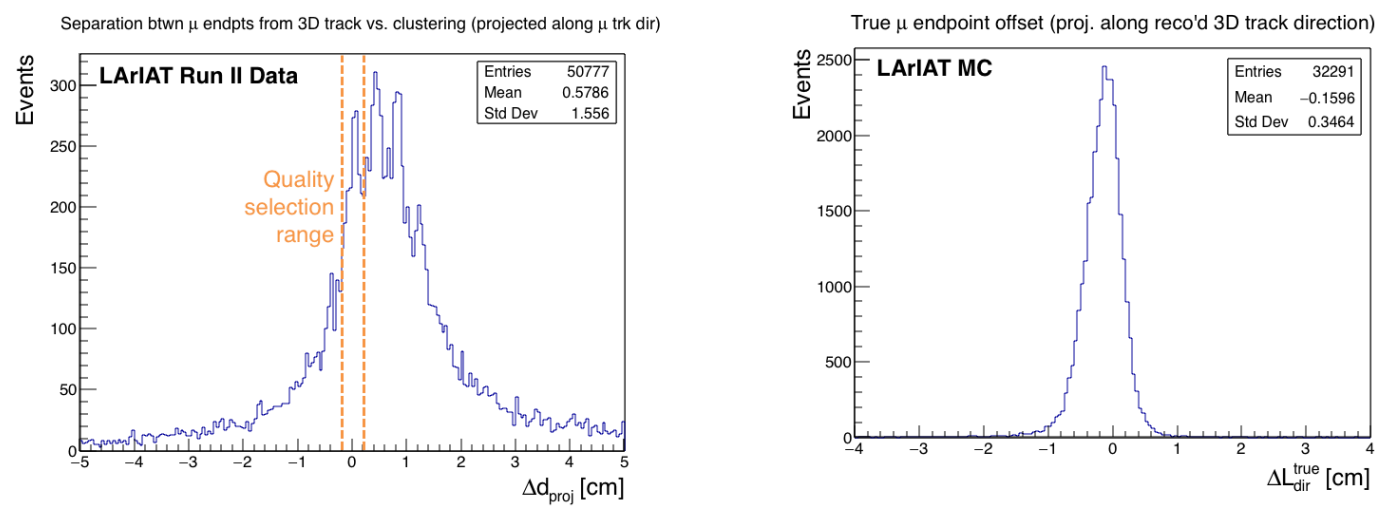

Figure 6.2: Distribution from data (left) of separations between the reconstructed endpoint of the stopping muon track and the 3D Michel boundary point identified through the custom clustering procedure, projected along the direction of the reconstructed 3D track. On the right is the difference (in MC) between the reconstructed and true muon endpoint, projected along the reconstructed track direction, for events passing the quality selection cut illustrated by the orange dashed lines on the left plot.

track $\vec{V}_{t r k}$, is less than $2 \mathrm{~mm}$ :

$$
-2 \mathrm{~mm}<\left(\mathbf{P}_{\text {bnd }}-\mathbf{P}_{\text {trk }}\right) \cdot \vec{V}_{\text {trk }}<2 \mathrm{~mm}
$$

Figure 6.2 (left) shows a distribution of this projected separation metric over a sample of Run II data events. The dashed orange lines indicate the bounds of the quality selection cut. The right plot in Figure 6.2 shows the true muon endpoint resolution projected along the track direction, $\left(\mathbf{P}_{\text {trk }}-\mathbf{P}_{\boldsymbol{\mu}}^{\text {true }}\right) \cdot \vec{V}_{t r k}$, for the events passing the endpoint quality cut described above. This distribution indicates that, on average, the reconstructed muon endpoint stops short of the true muon endpoint by about $1.6 \mathrm{~mm}$ with an RMS spread of $3.5 \mathrm{~mm}$.

- Measuring $\mathbf{d Q} / \mathbf{d x}$ Once we have selected a well-reconstructed track, $d Q / d x$ (corrected for $\tau_{e}$ ) and residual range $r$ along the track is recorded. Hits are only used if they have an inclination angle (relative to the electric field) of $\theta_{i n c}>20^{\circ}$ to match the sample used by ArgoNeuT in their parameterization of the Modified Box recombination model [58]. The hit's pitch $(d x)$ must also be less than $1.2 \mathrm{~cm}$. Hits' $d Q / d x$ fill a 


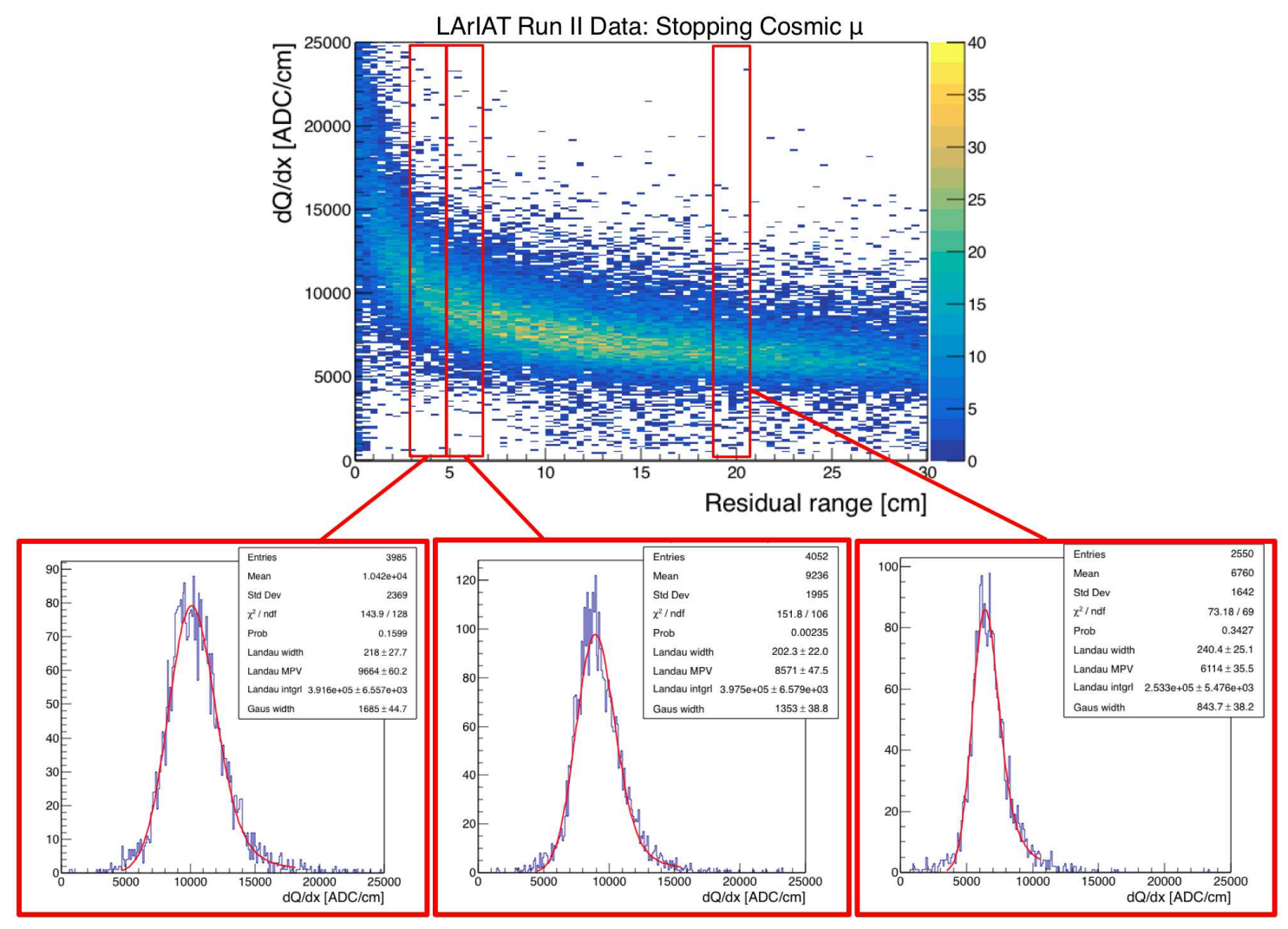

Figure 6.3: Reconstructed $d Q / d x$ and residual range along selected stopping muon tracks. Shown below this histogram are several example fits to $d Q / d x$ using a Landau convoluted with a Gaussian.

2D histogram with $r$ on the $\mathrm{X}$-axis and $d Q / d x$ in units of $\mathrm{ADC} / \mathrm{cm}$ on the $\mathrm{Y}$-axis.

Slices of this 2D histogram are made along the residual range axis in range $3-25 \mathrm{~cm}$, and projections of $d Q / d x$ for each group of residual range bins are made. Each distribution is fit to a Landau function convoluted with a Gaussian as illustrated in Figure 6.3.

- Fitting to predicted $\mathbf{d Q} / \mathbf{d x}$ : We need to know the energy of the muon at each point along its track in order to calculate the predicted MPV for energy deposition density, $(d E / d x)_{\text {pred }}$. To do this, we take calculated values of the mean $d E / d x$ for different residual ranges from a Particle Data Group (PDG) table for muons in liquid argon [91] and fit them to a simple power law function,

$$
d E / d x=A r^{b}+C
$$




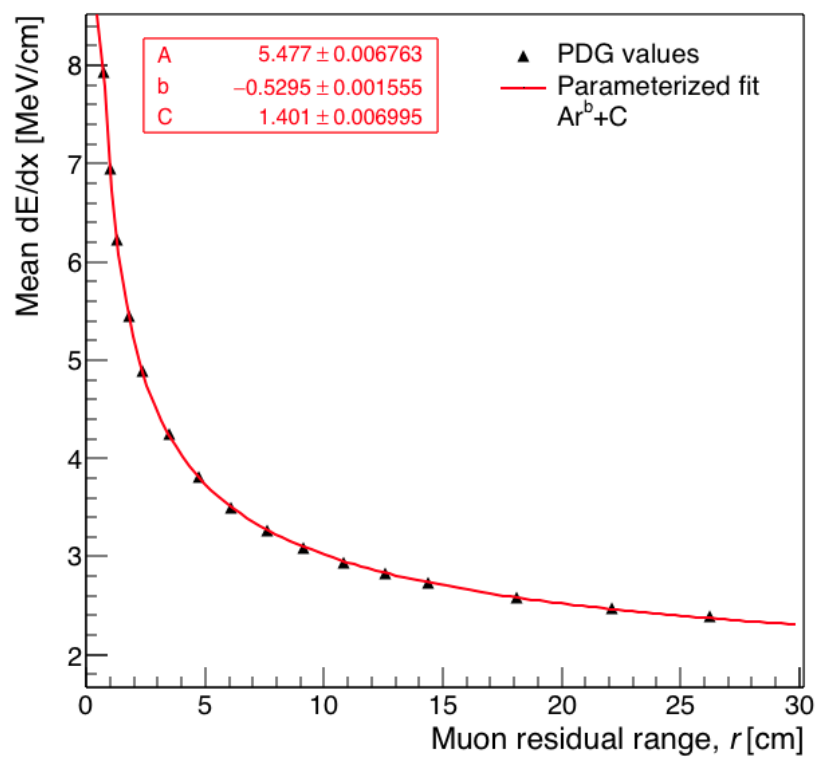

Figure 6.4: Mean $d E / d x$ versus residual range for muons. Points are taken from the Particle Data Group (PDG) table for muons in liquid argon [91]. The red curve is a parameterized fit of the form $d E / d x=A r^{b}+C$.

where $r$ is the residual range (in $\mathrm{cm}$ ) and $A, b$, and $C$ are treated as free parameters. This fit is shown in Figure 6.4. The fit results are

$$
\begin{aligned}
A & =5.477(7) \mathrm{MeV} / \mathrm{cm} \\
b & =-0.5295(16) \\
C & =1.401(7) \mathrm{MeV} / \mathrm{cm}
\end{aligned}
$$

By integrating Equation 6.3 over the muon's trajectory starting from its end point (where $r=0$ ), we derive a relationship for its energy as a function of its range,

$$
T_{r}=\left(\frac{A}{b+1}\right) r^{b+1}+C r
$$

This relation allows us to use Equation 3.3 to calculate the expected $d E / d x$ MPV, $(d E / d x)_{\text {pred }}$, at all points along the stopping muon's track, assuming some average 
LAr thickness $x$ (i.e., hit separation distance in 3D). For this study we use a thickness of $x=0.67 \mathrm{~cm}$ corresponding to the average hit pitch in Run II data for selected muon tracks. The value of $(d E / d x)_{\text {pred }}$ assigned to each group of residual range bins is determined as the mean of values calculated at both ends of the range: $(d E / d x)_{p r e d}^{r}=$ $\left[(d E / d x)_{\text {pred }}^{r 1}+(d E / d x)_{p r e d}^{r 2}\right] / 2$.

The predicted $d Q / d x$ (in $\mathrm{ADC} / \mathrm{cm}$ ) for any $d E / d x$ can then be calculated as

$$
d Q / d x[\mathrm{ADC} / \mathrm{cm}]=C_{c a l} \times \frac{d E / d x}{W_{\text {ion }}} \times R(d E / d x, \mathscr{E})
$$

where $C_{c a l}$ is the calibration constant $\left(\mathrm{ADC} / e^{-}\right), W_{\text {ion }}$ is the average ionization energy for liquid argon, and $R$ is the electron-ion recombination survival fraction.

Equation 6.8 is fit to the data, using as data points the MPV from the Landau fits to $d Q / d x$ at different residual ranges. The residual range offset found from $\mathrm{MC}$ in the right plot of Figure $6.2\left(r_{0}=-0.16 \mathrm{~cm}\right)$ is taken into account. The Modified Box recombination model is assumed, with its parameters fixed to those found by ArgoNeuT [58]. Errors on $d Q / d x \mathrm{MPV}$ are taken directly from the fits, while the error on $(d E / d x)_{\text {pred }}$ is based on the expected variation due to uncertainty in the muon endpoint projected along the muon track direction as found from $\mathrm{MC}\left(\sigma_{r}=0.35 \mathrm{~cm}\right)$. The best fit value for $C_{c a l}$ is taken as the final electronics response calibration result. These fits are displayed in Figure 6.5.

\subsection{Light-based calibrations}

We now turn to calibrations needed for scaling of scintillation-related quantities. These include (1) measuring the effective late-light lifetime; (2) determination of the single photoelectron response amplitude. 


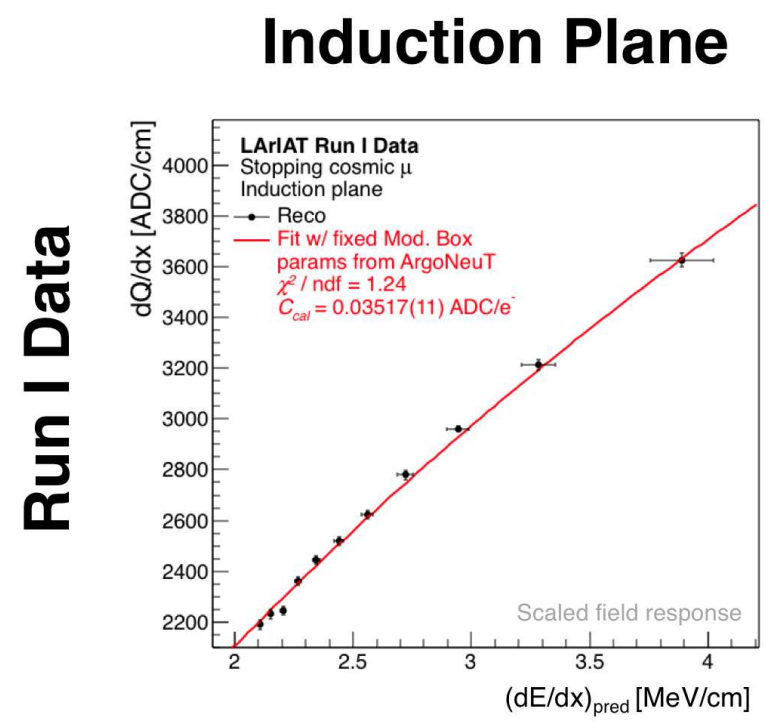

\section{Collection Plane}
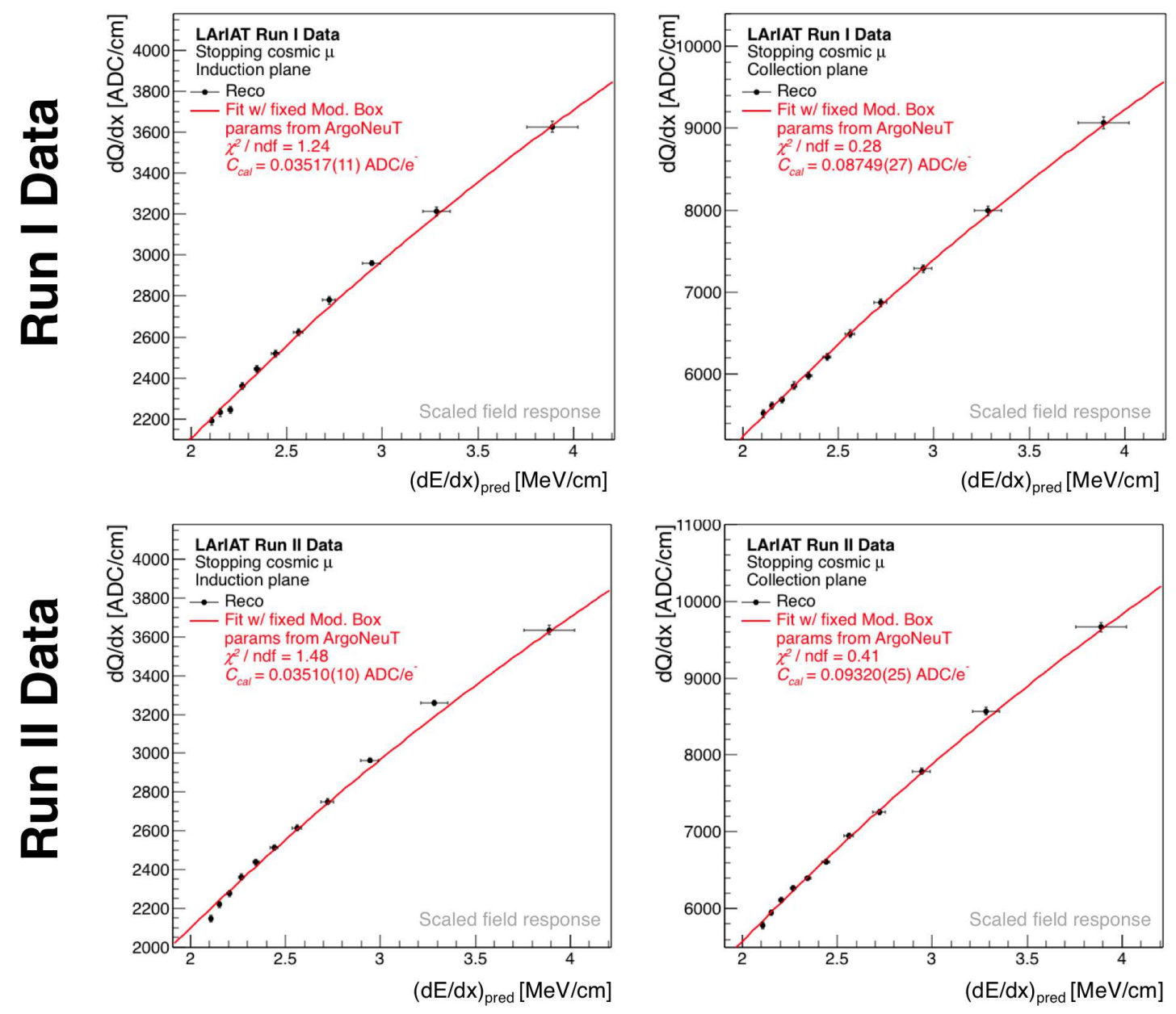

Figure 6.5: Charge response calibration fits for Run I and Run II using stopping muon tracks. 


\subsubsection{Effective late-light lifetime}

In order to correct for (and properly simulate) light quenching, information about the effective lifetime $\tau_{t}^{\prime}$ of the triplet-state $\mathrm{Ar}_{2}^{*}$ must be determined. As discussed in Chapter 3 and Appendix A, the actual lifetime we observe in scintillation signals from the PMTs is the result of a convolution of the Ar excimer decays, physical propagation, and (most importantly) the reemission fluorescence of TPB shown in Table 7.2 [67. A study using MC was carried out to derive a simple relationship between $\tau_{t}^{\prime}$ and $\tau_{\text {meas }}$, the measured late-light lifetime one would find from an averaged waveform (Equation A.1).

Averaged PMT waveforms for the event samples were created, with the samples split up into groups of 2-3 days in length. The lifetime $\tau_{\text {meas }}$ was found by fitting the inverted muon-candidate pulse with a falling exponential in the range $0.4-2 \mu \mathrm{s}$. These are plotted in Figure 6.7. It is not clear why $\tau_{\text {meas }}$ is observed to be different for the two PMTs in Run IIB, though it is likely due to the HMM PMT's gain being set too low during this period. This difference could also be due to residual effects from the signal overshoot in the HMM which may reduce the late-light lifetime measured with this PMT (corrections to the overshoot are discussed in Section 5.4 .

\subsubsection{Single photoelectron response amplitude}

The single photoelectron (SPE) response determines the factor needed to convert integrated charge on the PMTs, in units of ADCs, into the corresponding number of photons that produced the signal. This value is found using a common single photoelectron (PE) calibration technique in which the integrals of single PE pulses are added to a histogram and fit to multiple Gaussians representing the pedestal (noise) hits as well as separate response distributions expected for the detection of $1 \mathrm{PE}, 2 \mathrm{PE}, 3 \mathrm{PE}$, etc. The mean of the Gaussian corresponding to $1 \mathrm{PE}$ is taken as the $\mathrm{SPE}$ response.

In LArIAT this calibration is done in-situ by using single PEs in the late-light tails of scintillation pulses where PEs are likely to be well-isolated in time. Each dataset was divided 
into a series of approximately 3-day-long subsamples, and the SPE was calculated for each subsample using an algorithm which scans the PMT signal waveform and searches for small PE-like pulses in regions of the waveform which are sufficiently quiet. Quiet regions are defined as periods of sustained dead-time (i.e., no samples are observed exceeding $3 \times$ the local signal RMS for some configurable length of time, usually 100 samples).

In Run I, there was substantially more electronic RMS noise on the optical signals, which made the SPE Gaussian difficult to resolve. This was improved in Run IIB by replacing one of the NIM fan-out modules which resulted in much more easily-resolved SPE responses for this run period as seen in Figure 6.6. The measured SPE responses over the two Michel electron datasets are plotted in Figure 6.7. This calibration was performed only for select periods of time in which data was collected for use in specific light-based studies, including the Michel electron calorimetry study presented in this thesis. A full calibration profile over
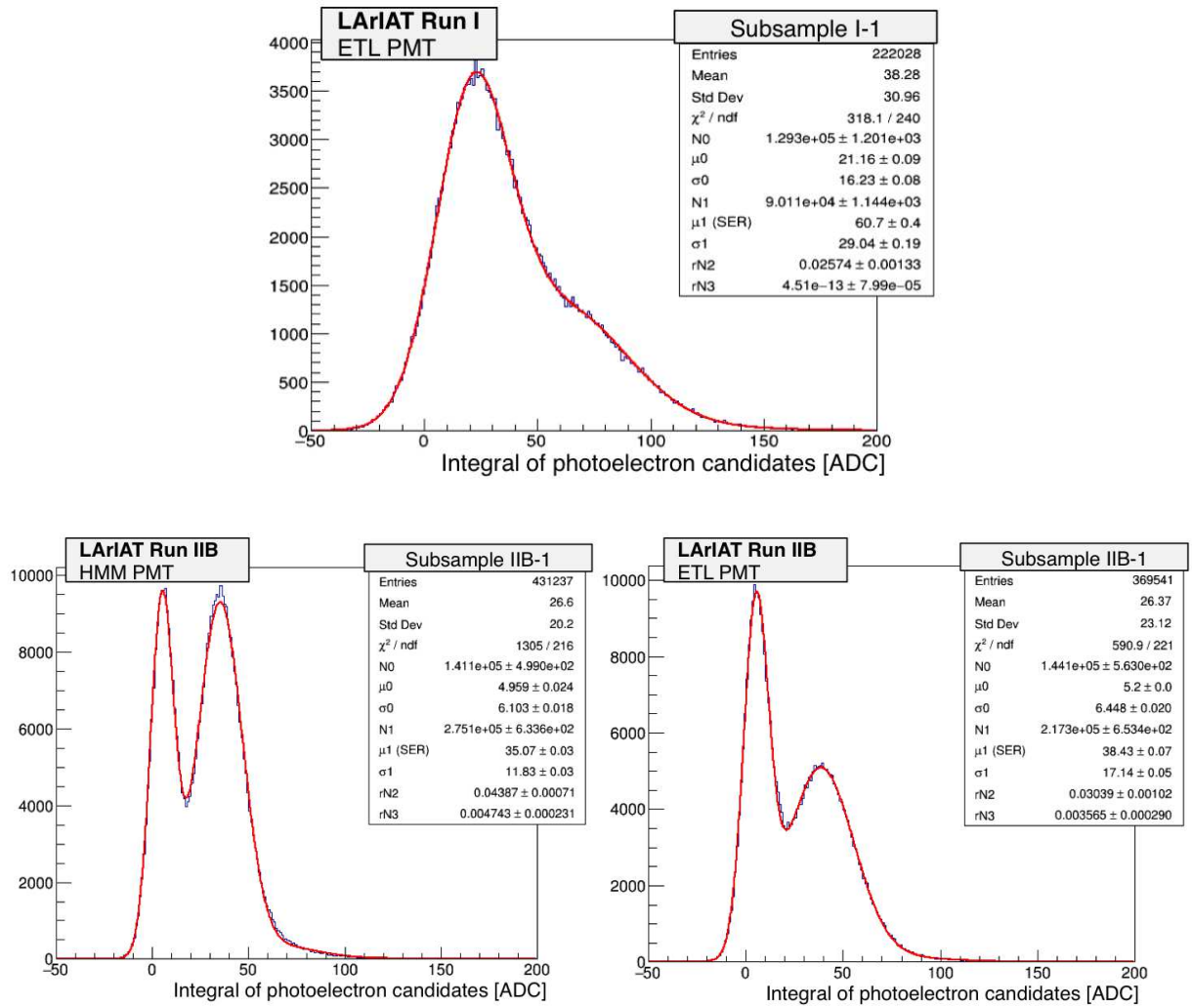

Figure 6.6: Examples of SPE calibration histograms for subsamples from the ETL PMT in Run I (top) and for both PMTs in Run IIB (bottom). 

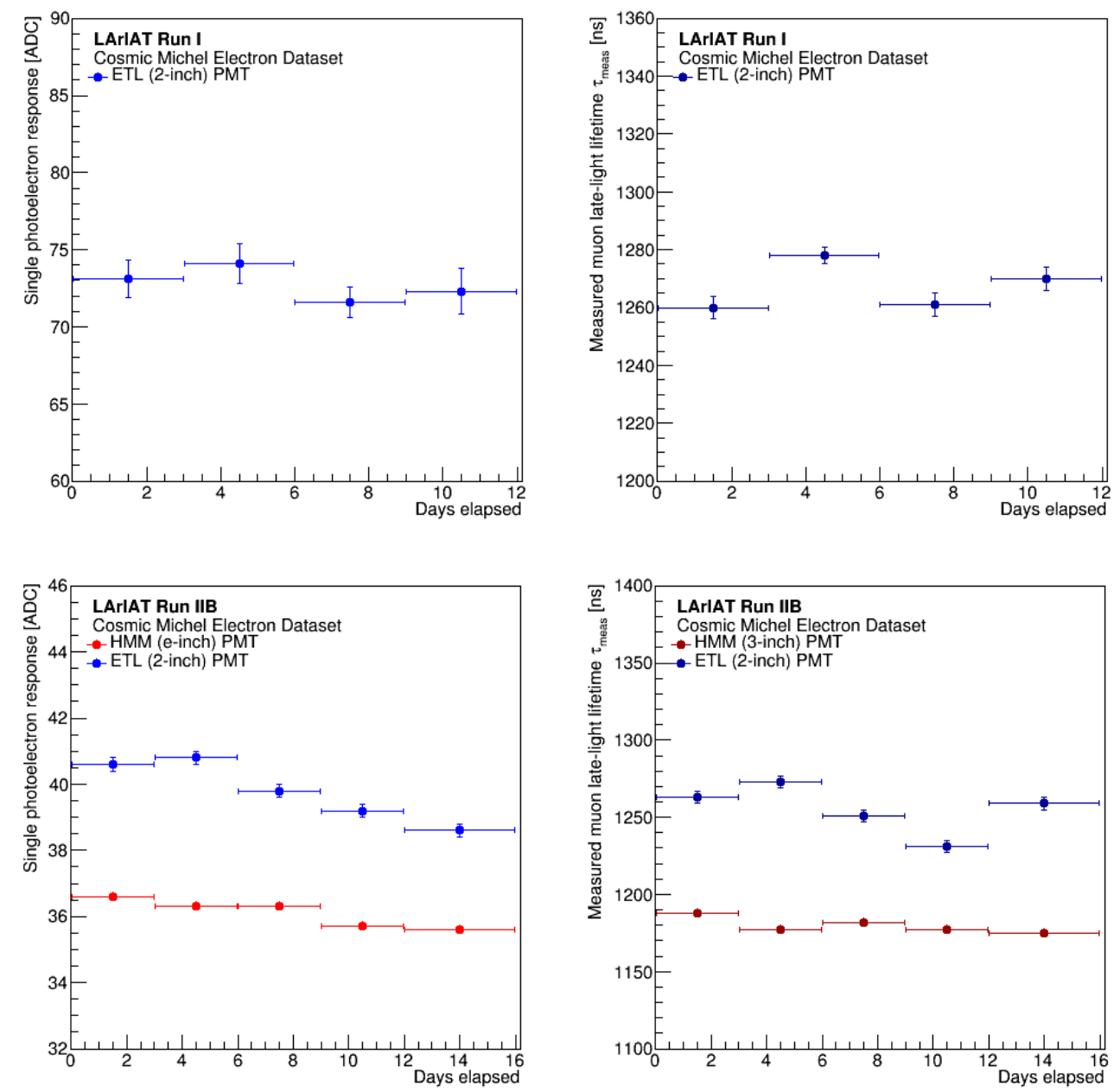

Figure 6.7: On the left are plots of the SPE response measured over time for the Run I (top) and Run IIB Michel electron datasets. On the right, $\tau_{\text {meas }}$ extracted from fits to the average waveforms for both datasets. Vertical error bars in both SPE response and $\tau_{\text {meas }}$ plots represent the uncertainty on parameters returned by fits. 
the entirety of Run I and Run II has not been carried out.

The advantage of this in-situ calibration is that it provides direct measure of the PMT gain over time in experimental data-taking conditions. Therefore, any effects on our signals from the cryogenic temperature and signal propagation through cables is reflected in the constant we extract. This is a useful way to monitor PMT gains over time so that the highvoltage can be adjusted if needed. For example, the gain in both PMTs is seen to gradually decrease over time in Figure 6.7, which prompted adjustments in the PMT HV for the period of time immediately following this (which is not included in these plots). 


\section{7}

\section{Monte Carlo Simulation}

An accurate simulation is critical for assessing our achieved energy resolution. In this chapter, the Monte Carlo (MC) simulation of the LArIAT detector is described in detail. First we describe how the scintillation photon visibility is mapped out using a photon propagation MC. Then we discuss the propagation of particles through our simulated geometry and simulation of energy loss, as well as the replication of charge and scintillation light signals. Finally, the effect from the inherent bias in the light-based trigger toward more luminous events is taken into account.

\subsection{Simulating photon visibility}

Scintillation light plays a prominent role in the analysis of low-energy electrons presented in subsequent chapters. However, light is complicated to reconstruct due to its propagation. Unlike ionization electrons, which drift along electric field lines in straight paths to the anode plane wires (suffering from exponential attenuation from impurity quenching, which is easily corrected using the arrival time), scintillation photons emanate isotropically from the interaction and undergo scattering and reflections. An overwhelmingly large fraction of photons produced by ionizing particles are never even collected by a photodetector. Understanding the distribution of photon visibility within our detector - i.e., the fraction of generated photons that are expected to reach a photodetector - is therefore important for reconstruction purposes. Light collected in an event needs to be scaled up by the inverse of the event's average visibility to account for losses during physical propagation.

In addition, a "map" of this visibility is used when simulating scintillation signals. Detailed Geant-level simulation of photon propagation is computationally taxing given the $\sim O(40,000)$ VUV photons that need to be tracked for every $\mathrm{MeV}$ of deposited energy. A visibility map acts as a stand-in for the physical propagation stage, allowing us to determine 
the number of detected photons instantly by simply scaling the number of generated photons by the visibility corresponding to the location of that energy deposit (more on this in Chapter 8).

In this section we describe how the $3 \mathrm{D}$ photon visibility and arrival times are mapped across the active volume of LArIAT using a standalone $\mathrm{MC}$ simulation written in $\mathrm{C}++$. The dimensions and placement of the TPB foils along the four field cage walls were coded in to replicate the actual setup in LArIAT as closely as possible. The photocathode windows of the two PMTs are represented as virtual "disks" suspended $5 \mathrm{~cm}$ behind the wire planes. This code has the advantage of running faster than a full Geant-level simulation due to a less complicated geometry, less memory overhead, and parameterized particle behavior as will be described later. This method also allows for easier modification of various properties related to how (for example) reflections from TPB foils are modeled.

Each photon is first assigned an energy of $E=9.69 \mathrm{eV}$ with a random Gaussian spread of $\sigma=0.25 \mathrm{eV}$ corresponding to wavelength $\lambda=128 \pm 8 \mathrm{~nm}$ [44]. A random isotropic starting direction is also chosen. The determination of the photon velocity $v_{\text {vuv }}$ and Rayleigh scattering length $L_{R}$ are based on energy $E[\mathrm{eV}]$ and wavelength $\lambda[\mathrm{nm}]$, respectively, using parameterized functions:

$$
\begin{aligned}
v_{\text {vuv }}[\mathrm{cm} / \mathrm{ns}] & =66.9-5.846 E \\
L_{R}[\mathrm{~cm}] & =174.7-0.03725 \lambda^{2}+\left(1.855 \times 10^{-6}\right) \lambda^{4}
\end{aligned}
$$

The functional form for $v_{\text {vuv }}$ above was found from a linear fit in the neighborhood of $E \approx 9.7 \mathrm{eV}$ to a plot of photon velocities generated using the group velocity equation, $v_{g}=c /[n(E)+d n / d(\ln E)]$, where $n$ is the energy-dependent index of refraction for LAr. The parameterization of $L_{R}$ is a polynomial fit to recently reported values [95] (shown in Figure 7.1) in the neighborhood of $\lambda=128 \mathrm{~nm}$, with an average calculated scatter length of $62 \mathrm{~cm}$. 


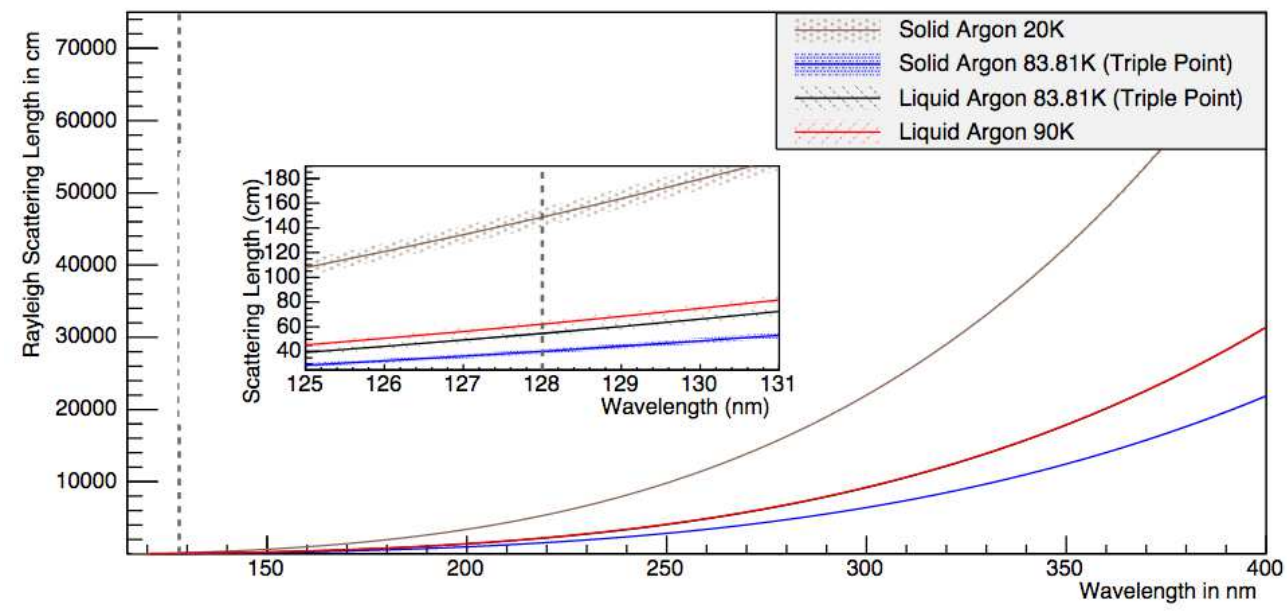

Figure 7.1: The calculated Rayleigh scattering length in liquid and solid argon as a function of wavelength taken from [95].

A photon is projected forward in a series of steps whose lengths are drawn randomly from the distribution, $e^{-x / L_{R}}$, based on the assigned Rayleigh attenuation length $L_{R}$. At each scattering point, a new direction is assigned using a random $\phi$ and a $\theta$ relative to the initial direction drawn from a distribution, $\left(1+\cos ^{2} \theta\right) \sin \theta$, that approximates scattering for low-energy photons. When a VUV photon reaches a TPB reflector foil, it has a chance of being wavelength-shifted to visible and assigned ${ }^{1}$ an energy $E=2.88 \mathrm{eV}$ corresponding to $\lambda \approx 430 \mathrm{~nm})$, velocity $v_{\text {vis }}=24 \mathrm{~cm} / \mathrm{ns}$, and Rayleigh scattering length $L_{R}=320 \mathrm{~m}$ [95]. Visible photons are propagated similarly through the active volume, undergoing scattering and reflections. This ray-tracing process continues until the photon fails a reflection efficiency cut or reaches a PMT face.

Reflections and reemission from TPB are assumed to be Lambertian with a $100 \%$ VUVto-visible conversion fraction and 95\% visible reflection efficiency [96]. Reflections from the copper cathode are described according to the GLISUR model [92] using a reflectivity of 0\% for VUV light and $17 \%$ for visible [97]. Wire plane shadowing is approximated for each plane using a parameterization of the transmission probability $P_{\text {trans }}$, motivated from geometrical arguments, which depends on the wire pitch $(p)$, wire diameter $(d)$, and the incoming photon

1. The variances in Rayleigh attenuation and velocity are negligible for visible light. Therefore we can safely neglect replicating the spread on assigned visible photon parameters. 
Table 7.1: Relevant parameters used in the standalone photon propagation Monte Carlo.

\begin{tabular}{|c|c|}
\hline Parameter(s) & Value \\
\hline PMT-wireplane clearance & $5 \mathrm{~cm}$ \\
\hline \multicolumn{2}{|l|}{ HMM PMT parameters } \\
\hline $\begin{array}{l}\text { - eff. photocathode radius } \\
\text { - window center coords. (x.v.z) }\end{array}$ & $\begin{array}{c}3.2 \mathrm{~cm} \\
(-5 \mathrm{~cm}, 0 \mathrm{~cm}, 47.9 \mathrm{~cm})\end{array}$ \\
\hline \multicolumn{2}{|l|}{ ETL PMT parameters } \\
\hline $\begin{array}{l}\text { - eff. photocathode radius } \\
\text { - window center coords. (x,y,z) }\end{array}$ & $\begin{array}{c}2.3 \mathrm{~cm} \\
(-5 \mathrm{~cm}, 0 \mathrm{~cm}, 40.7 \mathrm{~cm})\end{array}$ \\
\hline \multicolumn{2}{|l|}{ TPB film on PMT (ETL Run II) } \\
\hline - conversion efficiency & $100 \%$ \\
\hline - visible transmittance & $80 \%$ \\
\hline \multicolumn{2}{|l|}{ TPB reflector foils } \\
\hline - upstream/downstream dimensions & $l_{x}=43.18 \mathrm{~cm}, l_{y}=40.00 \mathrm{~cm}$ \\
\hline $\begin{array}{l}\text { - top/bottom dimensions } \\
\text { - conversion efficiency }\end{array}$ & $\begin{array}{c}l_{x}=43.18 \mathrm{~cm}, l_{z}=84.24 \mathrm{~cm} \\
100 \%\end{array}$ \\
\hline - visible reflectance & $95 \%$ \\
\hline - reflection model & Lambertian (diffuse) \\
\hline \multicolumn{2}{|l|}{ Copper cathode } \\
\hline - reflectivity to VUV & $0 \%$ \\
\hline - reflectivity to visible & $17 \%$ \\
\hline - reflection model & GLISUR \\
\hline - polish factor & 0.80 \\
\hline \multicolumn{2}{|l|}{ Wireplane shadowing } \\
\hline - num. planes & 3 \\
\hline - wire diameter (D) & $0.15 \mathrm{~mm}$ \\
\hline - wire pitch $(\mathrm{P})$ & $4 \mathrm{~mm}$ \\
\hline \multicolumn{2}{|l|}{ Visible photons } \\
\hline - Rayleigh length $\left(\mathrm{R}_{L}\right)$ & $320 \mathrm{~m} 95$ \\
\hline - velocity & $24 \mathrm{~cm} / \mathrm{ns}$ \\
\hline
\end{tabular}

angle projected onto the plane normal to the wires $(\theta)$ [98]:

$$
P_{\text {trans }}= \begin{cases}1-\frac{d}{p \cos \theta} & \cos \theta>d / p \\ 0 & \cos \theta \leq d / p\end{cases}
$$

No reflectivity is simulated at the glass windows of the PMTs, and photons are counted as detectable once arriving. All the relevant simulation parameters mentioned here are summarized in Table 7.1 . 
We now use this $\mathrm{MC}$ tool to generate $3 \mathrm{D}$ maps of the fractional visibility $\left(f_{\mathrm{vis}}\right)$ in LArIAT. We also encode some information about the arrival time of this light, though this is less critical for our analysis.

The active volume of the LArIAT TPC is divided into $24(\mathrm{x}) \times 20(\mathrm{y}) \times 45(\mathrm{z}) 3 \mathrm{D}$ voxels. Within each voxel, $N_{\gamma}^{\text {gen }}=300 \mathrm{~K}$ VUV photons are generated at random positions within the voxel boundary. The fraction of simulated photons reaching each photodetector, per voxel, is saved:

$$
f_{\text {vis }}(x, y, z)=\frac{\text { number photons generated at position }(x, y, z) \text { reaching PMT }}{N_{\gamma}^{\text {gen }}}
$$

where the $x y z$-position is taken as the center of the voxel. Metrics describing the time distribution of both visible and VUV photons - the minimum $\left(t_{\min }\right)$, average $\left(t_{\text {ave }}\right)$, and RMS $\left(t_{\text {rms }}\right)$ of arrival times - is also encoded in this map.

Projections in 2D of this simulated visibility map for both Run I and Run IIB are shown in Fig. 7.2. As we expect, the normalization of $f_{\text {vis }}$ throughout the volume is $\approx 5$ times higher in Run IIB compared to Run I since both PMTs were operational. Also, the TPB layer on the ETL in Run II is reflected in the maps through a region of relatively high $f_{\text {vis }}$ extending out from the position of the ETL, $x=40.7 \mathrm{~cm}$. Unfortunately, this dip-coated layer of TPB was not applied in a very systematic way, so its exact thickness is unknown. Estimated guesses of its transparency to visible light (80\%) were used in the simulation as shown in Table 7.1.

It is worth noting that $f_{\text {vis }}$ encodes only information about the physical geometric propagation of the light within the detector. The time structure of LAr scintillation, TPB reemission, as well as quantum and collection efficiencies of the PMTs are taken into account during optical signal generation described later in Section 7.5 . 

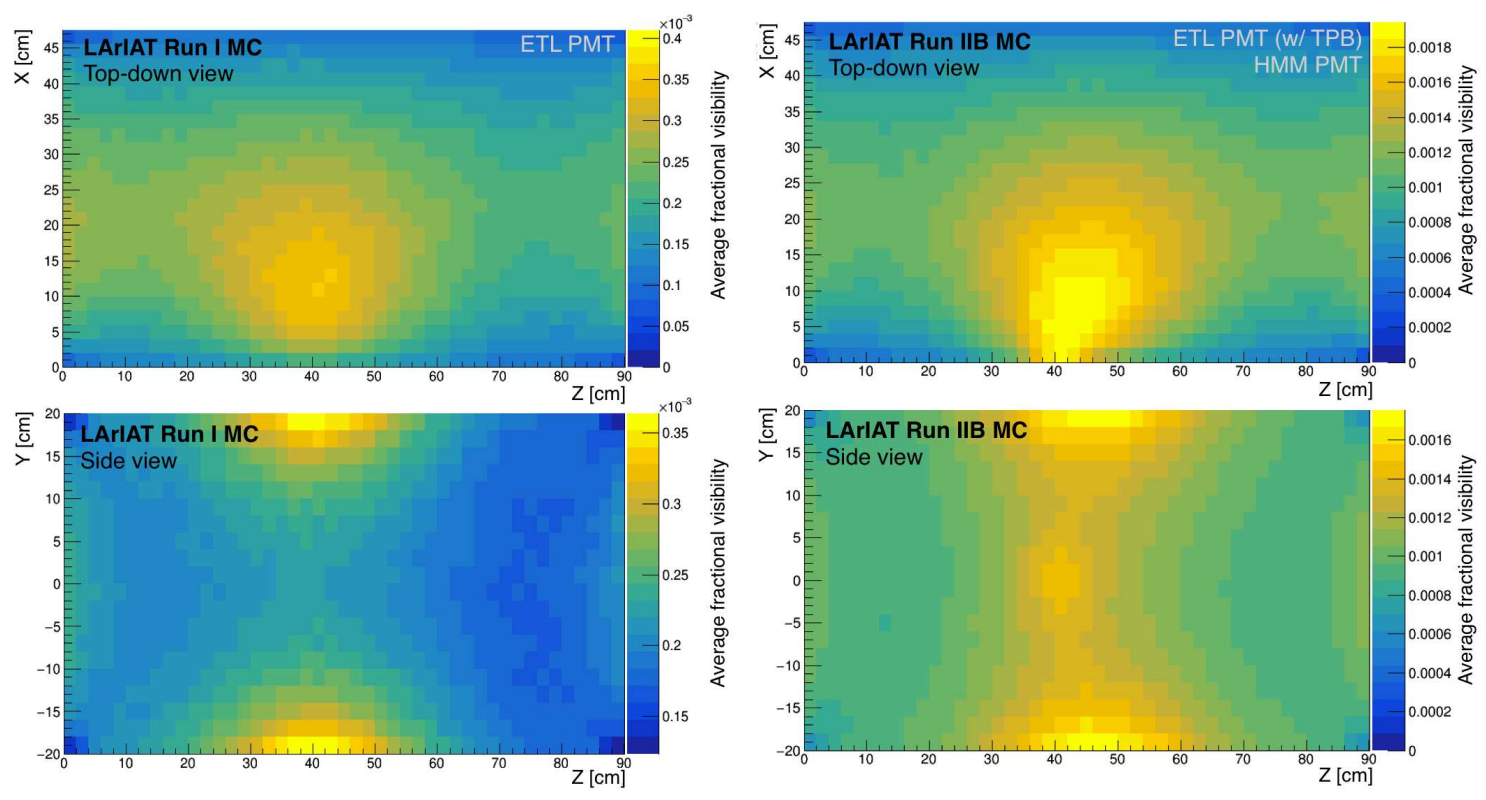

Figure 7.2: Projections of the average fractional scintillation visibility maps for Run I and IIB. Note the differences in color-axis scale between the two runs, with $f_{\text {vis }}$ averaging at $\approx 0.02 \%$ in Run I (where only the ETL PMT is used) and five times higher, $\approx 0.1 \%$, in Run IIB (where both PMTs are operational).

\subsection{Particle propagation and energy loss with Geant4}

We now turn to the actual simulation of charged particles. A sample of cosmic muons which stop and decay within the TPC is generated for comparison to data using LArSoft [78. The initial properties of each simulated muon are determined as follows:

1. A random point is chosen in the TPC.

2. An incoming muon direction vector is then determined by drawing a zenith angle from a $\cos ^{2} \theta$ distribution with a random $\phi$. From the randomly chosen point, we walk backward along this direction vector to the point $5 \mathrm{~cm}$ beyond the TPC boundary, and assign this point as the muon starting location.

3. The muon is assigned a momentum vector of random magnitude $|p|=50-300 \mathrm{MeV} / \mathrm{c}$, aimed toward the point in the TPC chosen earlier. The charge ratio of the simulated $\mu$ flux is set to $\mu^{+} / \mu^{-}=1.25$ based on a measurement by CMS [93] which is confirmed by LArIAT data in Section 5.5 . 

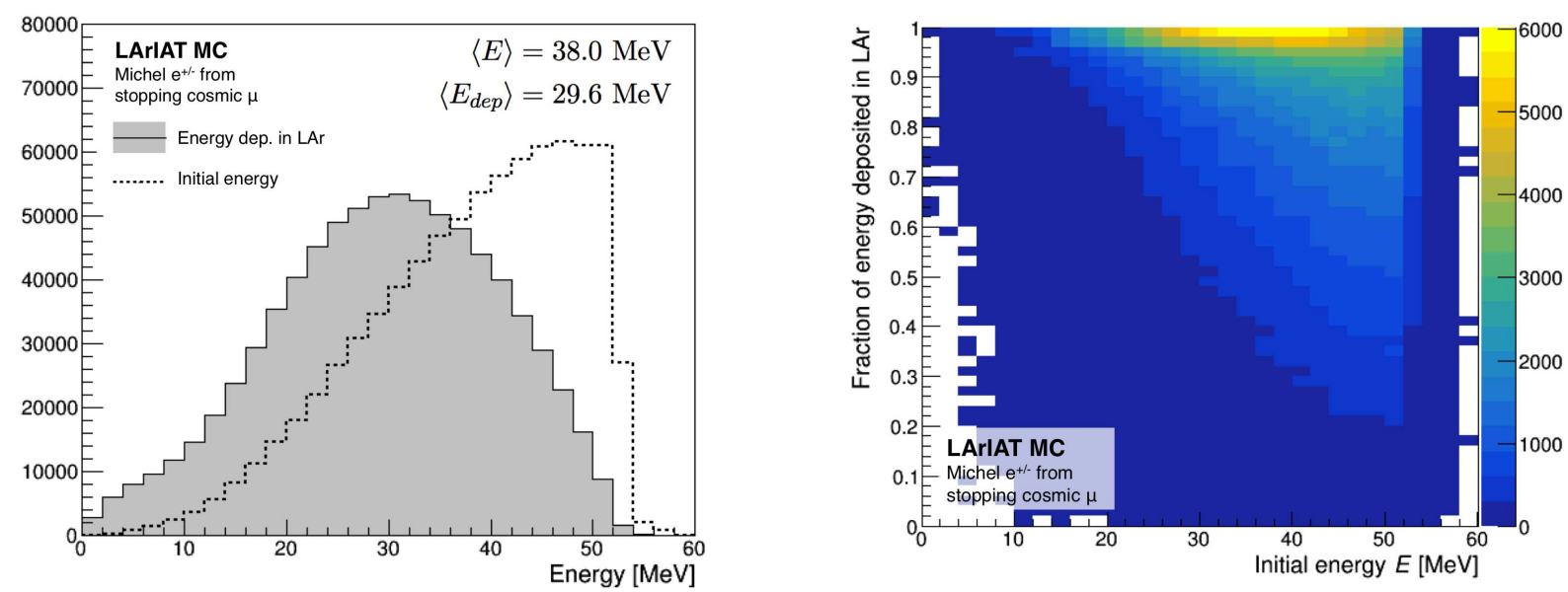

Figure 7.3: True energy and deposited energy distributions for Michel electrons in the $\mathrm{MC}$ sample prior to any trigger efficiency cuts (left), and the energy containment fraction, $E_{d e p} / E$, plotted against the initial Michel electron energy $E$ (right).

Geant4 [92], incorporated into the broader LArSoft framework, is used to propagate the muons (and their decay electrons) through the LArIAT geometry and simulate energy loss. The energy loss is calculated in accordance with the principles outlined in Chapter 3 . Separate MC samples were produced for Run I and Run IIB with detector properties set to match averages seen in the datasets.

Due to LArIAT's limited size, many bremsstrahlung photons emitted during the electromagnetic showering process escape the LAr active volume before pair-producing or Compton scattering, or the Michel electron itself will sometimes leave the TPC. Because these effects limit the detectable energy, the characteristic energy cut-off in the Michel spectrum is not resolvable without detailed containment corrections which are not attempted in this analysis. Figure 7.3 compares the distribution of true Michel electron energies and the energy deposited in the TPC.

\subsection{Charge and scintillation production}

The energy loss $(\Delta E)$ and step size $(d x)$ at each step of a particle's trajectory as simulated by Geant4 is passed onto native modules in LArSoft which use this information to determine 


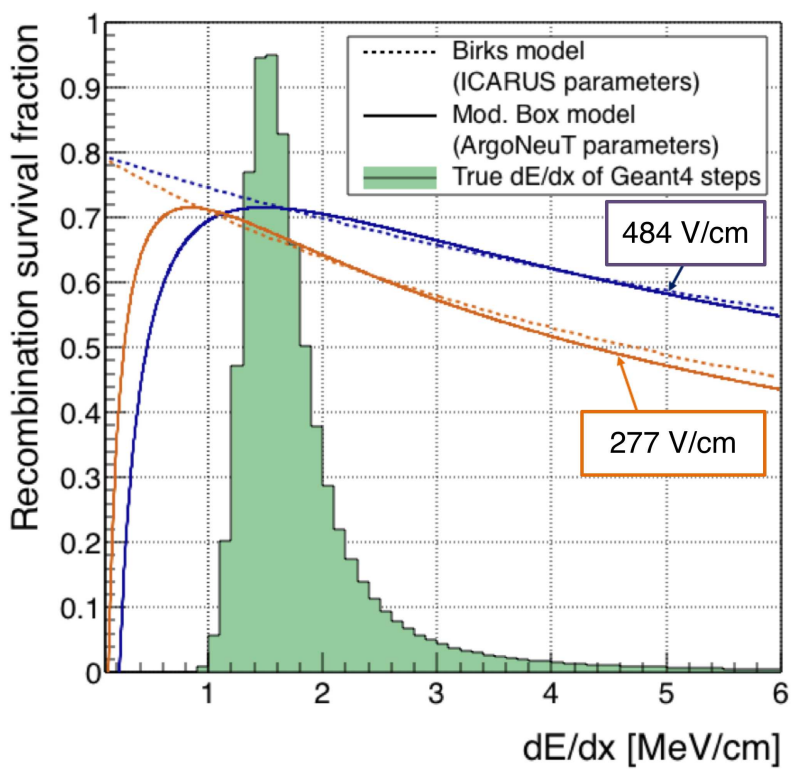

Figure 7.4: The Birks recombination model (dashed line) and Modified Box recombination model (solid line) as functions of $d E / d x$ for two different electric field values. Parameterizations from fits to data from the ICARUS [55] and ArgoNeuT [58] experiments are used. An arbitrarily-normalized distribution of $d E / d x$ for Geant4 particle steps is overlaid.

the quantity of free ionization electrons and scintillation photons to be produced.

Deposited energy $\Delta E$ is first divided into populations of $\operatorname{Ar}_{2}^{*}$ excimers $\left(N_{\mathrm{ex}}\right)$ and electronion pairs $\left(N_{\mathrm{i}}\right)$ according to the excitation ratio in LAr, $\alpha=N_{\mathrm{ex}} / N_{\mathrm{i}}=0.21$ [41, 43]. The $d E / d x$ of the particle step determines the fraction of free electrons surviving recombination $(R)$ based on both parameterized models described in Section 3.2. The Modified Box model (Equation 3.12 is applied for $d E / d x \gtrsim 1.7 \mathrm{MeV} / \mathrm{cm}$ and the Birks model (Equation 3.9) is applied for small- $d E / d x$ to avoid the unphysical downturn in the Modified Box model for LArIAT's electric field as plotted in Fig. 7.4. The exact transition between these two regimes is the point in the neighborhood of low- $d E / d x$ at which the models intersect, $\approx$ $1.65 \mathrm{MeV} / \mathrm{cm}$.

The final number of photon-producing $\operatorname{Ar}_{2}^{*}\left(N_{\gamma}\right)$ is determined from Eq. 3.15 such that 
the anti-correlation expected between $N_{\gamma}$ and $N_{e}$ is preserved after recombination:

$$
\begin{aligned}
& N_{e}=N_{\mathrm{i}} R \\
& N_{\gamma}=N_{\mathrm{ex}}+N_{\mathrm{i}}(1-R) .
\end{aligned}
$$

Excimers are then divided into fast (singlet) and slow (triplet) populations using ratios from literature. A singlet-to-triplet ratio of $I_{s} / I_{t}=0.51$ is applied for muon and electron-induced ionization while $I_{s} / I_{t}=0.3$ is used for photon-induced ionization [50, 94].

\subsection{Charge drift and signal formation}

The simulation of charge drift to the wireplanes is then replicated. Electrons produced at each step along the ionizing particle's path are each scaled down based on an electron lifetime $\tau_{e}$ which is provided as an input parameter to the simulation and set to match data (see Chapter 6),

$$
N_{e}^{\mathrm{drift}}=N_{e} \exp \left(-t / \tau_{e}\right)
$$

where $t$ is the charge deposit's drift time, calculated as $t=x / v_{e}$ where $x_{i}$ is the X-location of the deposited charge and $v_{e}$ is the electron velocity in LAr.

The distribution (in time) of arriving charge at each plane is then convoluted with the electronics response and field response functions (see Section 5.3.1) to generate signals on the wires. Noise is also added based on a noise frequency profile taken from data.

\subsection{Scintillation signal formation}

To simulate light quenching due to collisions with impurities (Section 3.3), first the effective

quenched triplet lifetime $\tau_{t}^{\prime}$ is used to calculate $\tau_{q}=\left(1 / \tau_{t}^{\prime}-1 / \tau_{t}\right)^{-1}$. The value of $\tau_{t}^{\prime}$ is calculated from $\tau_{\text {meas }}$ as described in Appendix A, which is provided as an input parameter to the simulation and set to reflect the average values from the datasets (see Chapter 6). 
Table 7.2: Measured TPB decay times and relative abundances (adapted from [67]). In the simulation, the instantaneous component (1-10 ns) is modeled as $\tau=5 \pm 5 \mathrm{~ns}$.

\begin{tabular}{|l|c|c|}
\hline & decay time [ns] & abundance (\%) \\
\hline Instantaneous component & $1-10$ & $60 \pm 1$ \\
Intermediate component & $49 \pm 1$ & $30 \pm 1$ \\
Long component & $3550 \pm 500$ & $8 \pm 1$ \\
Spurious component & $309 \pm 10$ & $2 \pm 1$ \\
\hline
\end{tabular}

This $\tau_{t}^{\prime}$ is used to calculate $\tau_{q}$, thus enabling the calculation of the effective singlet decay time, $\tau_{s}^{\prime}$, and the overall yield reduction factors necessary for proper simulation of $N_{\gamma}$ quenching. For typical measured late-light lifetimes in the data, $\tau_{\text {meas }} \approx 1200 \mathrm{~ns}\left(\tau_{t}^{\prime} \approx 1120\right.$ ns), we expect negligible quenching of the fast-decaying singlet population $\left(\tau_{s}^{\prime} / \tau_{s} \approx 1\right)$ and a $14 \%$ reduction in the slower-decaying triplet population $\left(\tau_{t}^{\prime} / \tau_{t} \approx 0.86\right)$.

Once quenching of the two excimer populations has been applied, the physical propagation of the photons to the PMTs must be simulated. This process is folded into the visibility map constructed in Section 7.1. For each PMT, the fractional visibility $f_{\text {vis }}$ corresponding to the point of charge and light deposition is retrieved from the visibility map. The quantum and collection efficiencies of the PMTs themselves are then folded into this factor to arrive at an effective scale factor, $f_{\mathrm{eff}}=f_{\mathrm{vis}} \times f_{\mathrm{QE}} \times f_{\mathrm{CE}}$. The number of photons produced by the energy deposit, $N_{e}$, is then scaled by factor $f_{\text {eff }}$ to yield the number of detected photoelectrons, drawing from a binomial distribution to include stochastic fluctuations. Timing information from the map is also retrieved and each photon is assigned a physical propagation time $t_{\text {prop }}$ drawn randomly from a Gaussian of $\mu=t_{\text {mean }}$ and $\sigma=t_{\text {rms }}$ (if $t_{\text {prop }}<t_{\text {min }}$ a new random time is drawn). The resulting photon arrival time is then a convolution of the particle's birth time, the quenched LAr scintillation lifetimes $\tau_{s}^{\prime}$ and $\tau_{t}^{\prime}$, propagation time $t_{\text {prop }}$, and the TPB decay time constants shown in Table 7.2. 


\subsection{Optical smearing and trigger efficiency}

The PMT electronics response has not been modeled in our simulation. To reproduce detector resolution effects, we smear the integrated photoelectron counts from the Monte Carlo. This is done on an event-by-event basis by randomly varying the raw integrated counts, $N$, based on a two-component resolution factor,

$$
\sigma=\sqrt{\sigma_{N}^{2}+\sigma_{T}^{2}}=\sqrt{N \sigma_{p e}^{2}+T \sigma_{\mathrm{RMS}}^{2}}
$$

where $T$ is the integration time in nanoseconds. The two resolution factors, $\sigma_{p e}$ and $\sigma_{\mathrm{RMS}}$, are derived from data and described below:

- $\sigma_{N}=\sqrt{N} \sigma_{p e}$ : This factor, proportional to the square-root of the number of detected photoelectrons, approximates effects from the inherent width of the single photoelectron Gaussian peak from the PMT calibration histograms (see Section 6.2). The average SPE mean $\left(\mu_{1}\right)$ and width $\left(\sigma_{1}\right)$ are found for the individual PMTs in each of the two datasets, and the ratio of the two $\left(\mu_{1} / \sigma_{1}\right)$ is set as $\sigma_{p e}$ for the MC sample corresponding to each respective data sample.

- $\sigma_{T}=\sqrt{T} \sigma_{\mathrm{RMS}}:$ This factor represents the contribution from RMS noise in the PMT waveforms and is thus proportional to the square-root of the integration time in nanoseconds, $T$. We determine this value by integrating a 1000-ns window in the pedestal-subtracted baseline/pedestal region of all waveforms in data and extract the RMS of this distribution in units of PE. Then, following the assumed relationship $\sigma \sim \sqrt{T}$, we calculate $\sigma_{\mathrm{RMS}}=\mathrm{RMS} / \sqrt{1000}$

Figure 7.5 shows an example integrated baseline region used to calculate $\sigma_{\mathrm{RMS}}$ from data. The values of these smearing parameters calculated from each data sample are summarized in Table 7.3 .

We now turn to some additional modifications to the reconstructed light in MC which 


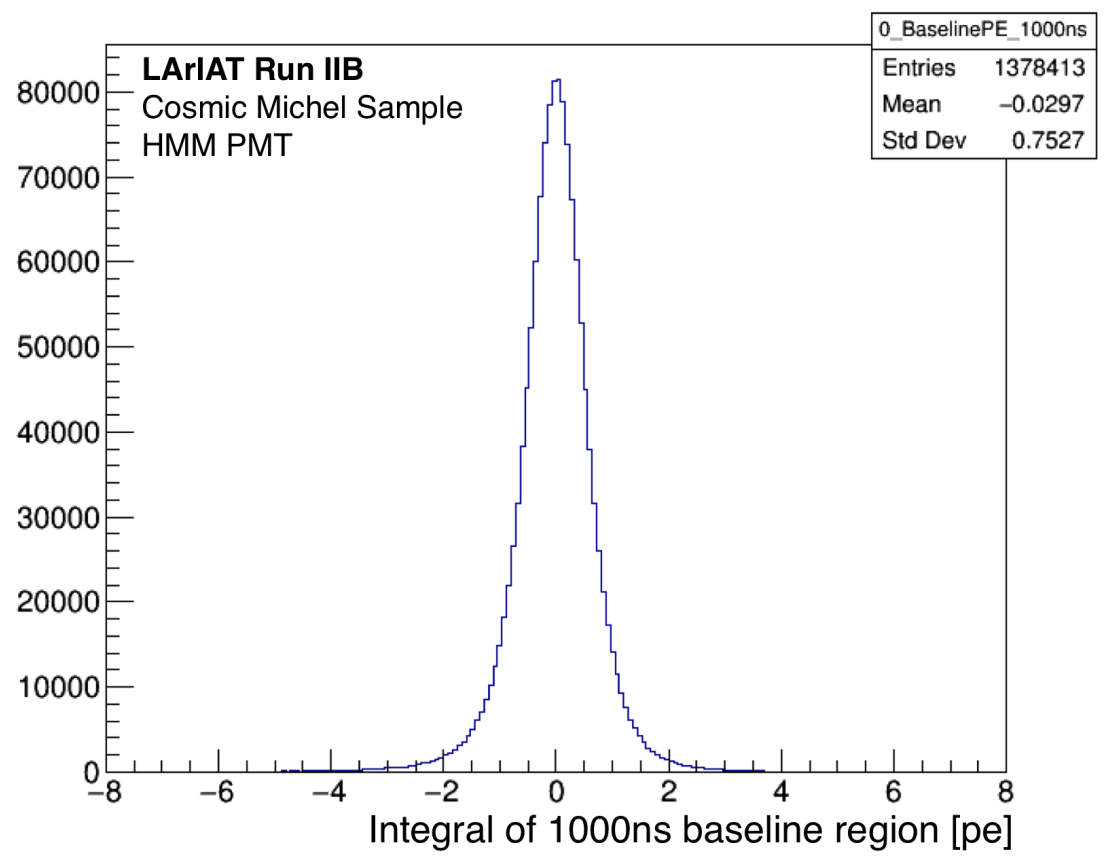

Figure 7.5: Integral of baseline region used to estimate the inherent electronics noise in the Hamamatsu PMT for Run IIB.

are determined by finding the best-match to the final distributions of prompt photoelectrons $\left(S_{100}\right)$ and total photoelectrons $(S)$ in data. These are discussed below:

- $\sigma_{X}[\mathrm{pe}]=\sigma_{x} \times S$ : This "extra" smearing contribution is applied only to the total light integral, $S$, and is meant to fold in other effects that might distort the data (like recurrent noise oscillations) which would be hard to model in a natural way using SPE resolution and waveform RMS noise. The effect of this extra smearing to the total light distributions is illustrated in Figure 7.6 .

- Trigger efficiency parameters $P$ and $K$ : As mentioned previously, the light-based trigger used to obtain our sample introduces a bias toward more luminous, higher energy Michel decays. To replicate this bias in our MC sample, we define a trigger efficiency function that returns a probability of accepting an event with prompt light $S_{100}$,

$$
f\left(S_{100}\right)=1-\frac{1}{1+\left(S_{100} / P\right)^{K}}
$$


Parameter $P$ dictates the approximate cut-off point in the prompt light distribution, while $K$ controls the sharpness of this boundary. A visual overlay of the (scaled) trigger efficiency functions with each prompt light distribution from the MC is shown in Figure 7.7 .

- Scaling: Given the number of tunable parameters and approximations used in the photon propagation simulation (reflection modeling, surface reflectances, wire plane transparency to visible light), combined with the uncertainty in the reported PMT collection efficiencies [73], we take the liberty of scaling the light distributions by some amount to match distributions in data. This is effectively treated as scaling of each PMT's global photoelectron detection efficiency.

These four parameters $-\sigma_{x}, P, K$, and the scale factor - are determined by finding values best matching data distributions after applying a decay time cut of $\Delta T>1.8 \mu \mathrm{s}$ and requiring the event to have a successfully reconstructed $2 \mathrm{D}$ shower. First the MC distributions of $S_{100}$ and $S$ are area-normalized to the total entries in the corresponding histograms for data. A chi-squared metric with bin content weighting is used to compute the goodness-of-match between histograms in data and their (normalized) MC counterpart histograms,

$$
\chi_{w}^{2}(\mathrm{~A}, \mathrm{~B})=\left(\sum_{i=0}^{N} a_{i}\right)^{-1}\left(\sum_{i=0}^{N} \frac{a^{i}\left(a^{i}-b^{i}\right)^{2}}{\left(\sigma_{a}^{i}\right)^{2}+\left(\sigma_{b}^{i}\right)^{2}}\right)
$$

where $\mathrm{A}$ and $\mathrm{B}$ are the data and MC histograms, $a^{i} / b^{i}$ the histograms' values at bin $i$, and $\sigma^{i}$ the error assigned to that bin. For data, $\sigma^{i}=\sqrt{a^{i}}$, but for MC the error is scaled down due to the area-normalization. For each PMT, the minimization package MINUIT in ROOT (using the SIMPLEX algorithm) is used to find the three-fold combination of $P, K$, and scale factor that simultaneously minimizes the $\chi_{w}^{2}$ of the prompt light distribution $S_{100}$. Then the minimization is performed over each total light distribution to find the optimal smear factor $\sigma_{x}$ with the previously-determined trigger efficiency parameters and scale factor fixed. The resulting distributions after optimization are overlaid with data in Figures 7.8 and 7.9 . 
Table 7.3: Smearing, scaling, and trigger-cut parameters applied to the MC samples. Also displayed are the data-MC $\chi_{w}^{2}$ comparison values after minimization for both prompt $\left(S_{100}\right)$ and total $(S)$ photoelectrons.

\begin{tabular}{|l|c|c|c|c|}
\hline Run Period & \multicolumn{2}{|c|}{ Run I MC } & \multicolumn{2}{c|}{ Run IIB MC } \\
\hline PMT & HMM & ETL & HMM & ETL \\
\hline$\sigma_{p e}[\mathrm{pe}]$ & - & 0.43 & 0.32 & 0.43 \\
$\sigma_{\mathrm{RMS}}[\mathrm{pe} / \sqrt{\mathrm{ns}}]$ & - & 0.043 & 0.024 & 0.024 \\
$\sigma_{x}[\mathrm{pe}]$ & - & 0.152 & 0.002 & 0.238 \\
Trig eff. $P[\mathrm{pe}]$ & - & 25.74 & 81.31 & 22.72 \\
Trig eff. K & - & 9.098 & 8.271 & 4.506 \\
Scale factor & - & 1.016 & 1.021 & 0.957 \\
$S_{100}$ data-MC $\chi_{w}^{2}$ & - & 2.88 & 1.01 & 0.53 \\
$S$ data-MC $\chi_{w}^{2}$ & - & 0.91 & 1.54 & 1.68 \\
\hline
\end{tabular}

The results of this minimization procedure are displayed in Table 7.3. Similar scale factors are required on the ETL PMT in Run I and the HMM PMT in Run IIB (+1-2\%). However, we find in Run IIB the ETL PMT needs to be scaled down by about $-4 \%$. This could be due to the TPB film that was applied via dip-coating to the window of this PMT in Run II. The exact conversion efficiency for VUV photons and the transparency to visible light of this TPB film is unknown, and in the simulation these values were estimated (see Table 7.1). 

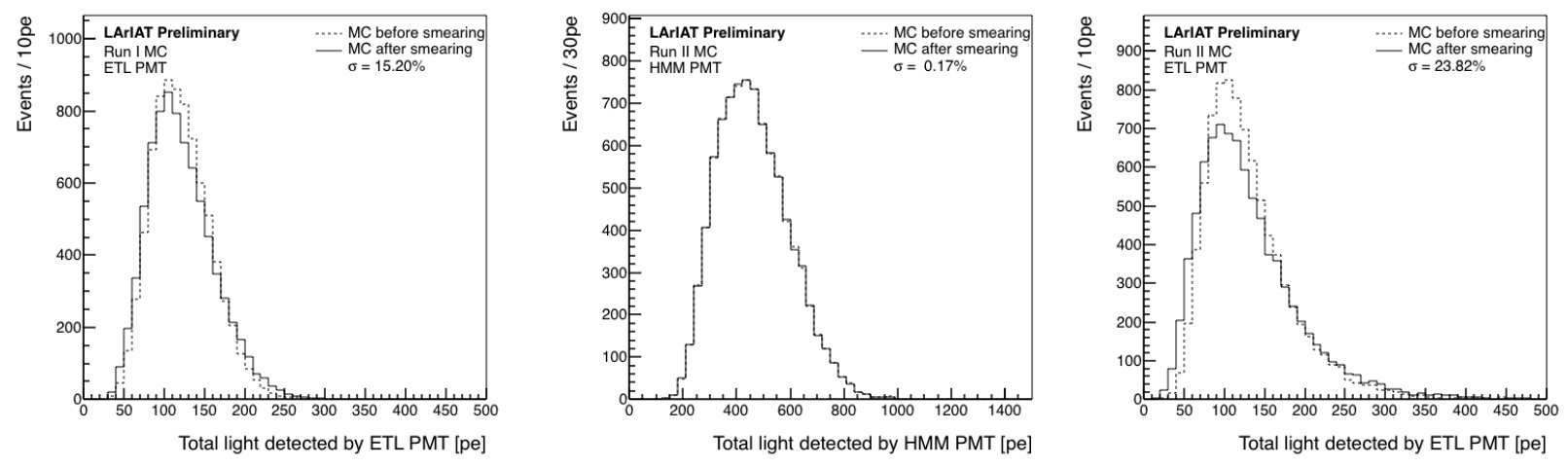

Figure 7.6: The effect of smearing contribution $\sigma_{X}$ on the total MC light distributions.
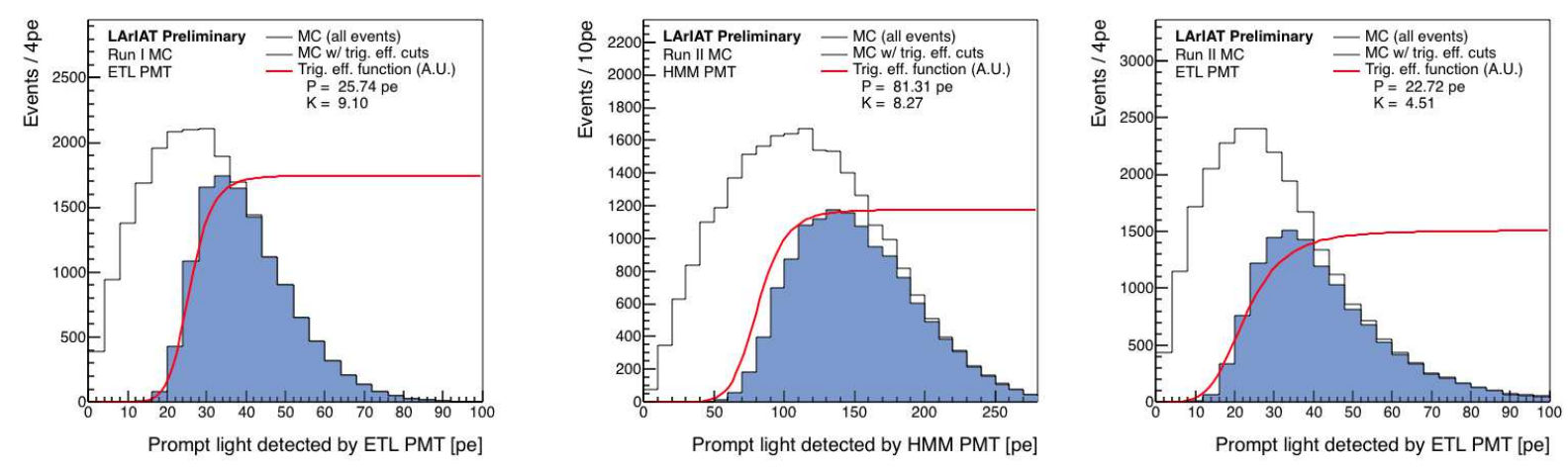

Figure 7.7: The trigger efficiency function (red) overlaid with its respective prompt light distribution for PMTs in both MC samples. In each case the function is scaled arbitrarily and is drawn only for illustrative purposes. The unfilled distribution shows the prompt light distribution for all events in MC prior to trigger cuts. 

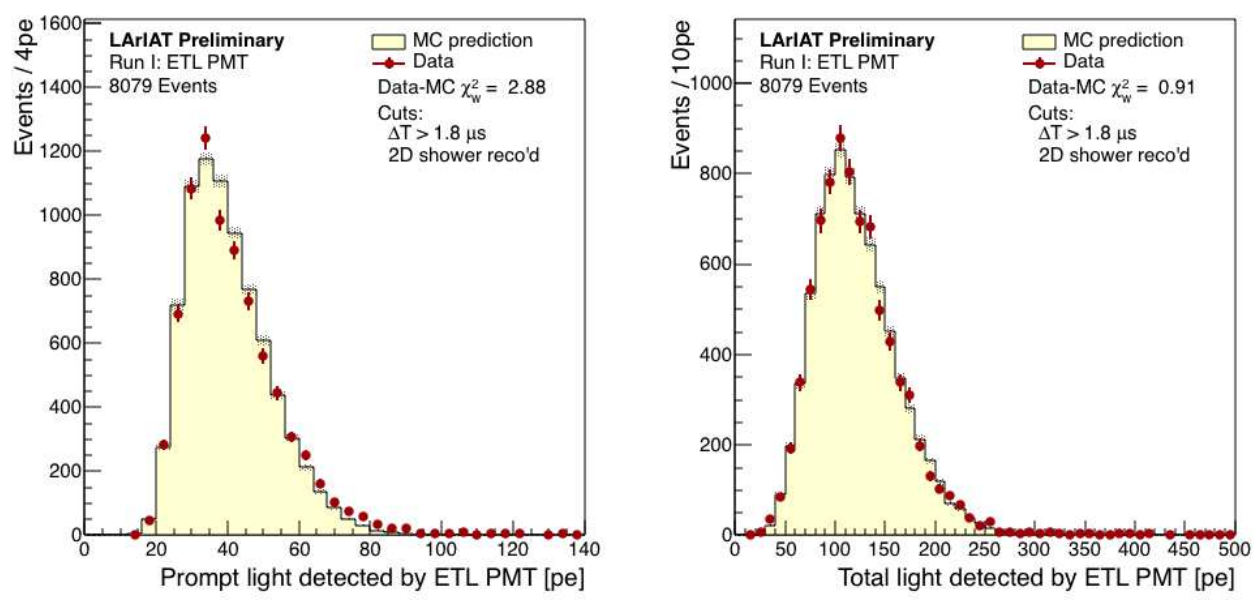

Figure 7.8: Run I distributions of $S_{100}$ (left) and $S$ (right) compared to MC after smearing, scaling, and trigger efficiency parameters are optimized.
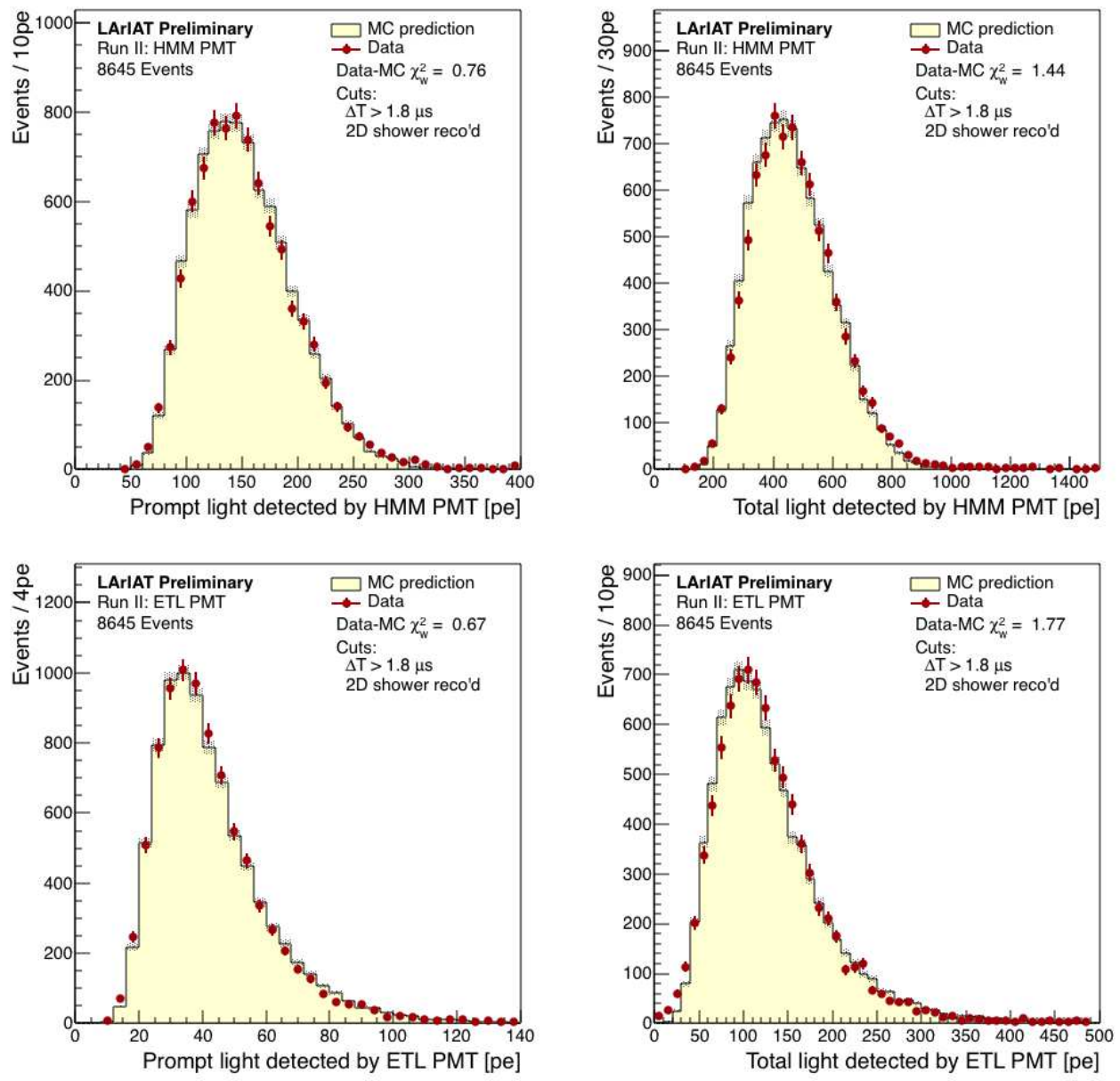

Figure 7.9: Run IIB distributions of $S_{100}$ (left) and $S$ (right) compared to MC after smearing, scaling, and trigger efficiency parameters are optimized. 


\section{Calorimetry Results for Low-Energy Electrons in LArIAT}

Now that we have obtained a reconstructed sample of Michel electrons and have verified the sample using the muon decay time (see Section 5.5), we turn now to calorimetry. First we provide a brief overview of the calculation of energy from light and charge, including the corrections needed on both quantities to compensate for attenuation effects and convert ADCs to physical quanta $\left(e^{-}\right.$or $\left.\gamma\right)$. We then present the reconstructed energy spectra for electron ionization tracks as well as full showers in Run I and Run IIB using the formulaic constructions of energy. Based on these results, the light yield (pe/MeV) of the LArIAT TPC is estimated for both Run I and Run II. Next, we prepare a maximum-likelihood fitter tailored specifically for the Michel electron sample in LArIAT's Run II and present the

resulting energy spectrum for comparison. MC resolution studies are carried out to gauge the improvement in Michel electron energy resolution using the three different reconstruction methods in Run II.

Following this, we use a sample of simulated electrons to determine LArIAT's energy resolution for isolated electron showers to better approximate the event topology expected from low-energy electron neutrino interactions.

\subsection{Overview of energy reconstruction methodologies}

Energies of Michel electron track and shower objects are reconstructed using three different methods as briefly described in Chapter 3. We outline these methods below. For the sake of clarity, quantities of charge $(Q)$ and light $(L)$ are assumed to represent the number of quanta, whether free electrons $\left(N_{e}\right)$ or photons $\left(N_{\gamma}\right)$, produced by the ionizing deposit.

- $\mathrm{E}_{\mathrm{Q}}$ : “charge-only" (Q-only) method. This is the traditional approach which relies 
only on the reconstructed charge, $Q$. Hits belonging to the electron or its displaced photon-initiated shower deposits on the collection plane are looped over and the total charge and charge-based energy are computed:

$$
E_{Q}=\left(Q \times R^{-1}\right) \times W_{\text {ion }}
$$

The recombination factor, $R=N_{e} / N_{\mathrm{i}}=0.69$, is chosen using the Modified Box model assuming an average Michel electron stopping power of $2.3 \mathrm{MeV} / \mathrm{cm}$. This assumption was used by MicroBooNE in their Michel electron analysis as well [34].

- $\mathrm{E}_{\mathrm{QL}}$ : "charge-plus-light" $(\mathrm{Q}+\mathrm{L})$ method. In this approach, the sum of the collected light and charge together is used to determine the energy deposited. For this method, only the full electron shower energy can be used since it is impossible to isolate (in time) the photoelectrons coming directly from the ionization of the electron itself. To combine light information, we first require that showers were constructed successfully on both planes. The 3D space points are looped over and the charge-weighted average visibility, $f_{\text {vis }}$, is calculated using the fractional visibility maps discussed in Section 7.1. The total scintillation light, $L$, is then calculated from the detected light, S. A scaling is also performed on the late component of the scintillation (ie, $>100 \mathrm{~ns}$ ) to correct for quenching effects. We then calculate the $\mathrm{Q}+\mathrm{L}$ energy as

$$
E_{Q L}=(Q+L) \times W_{\mathrm{ph}}
$$

Due to the complementarity of light and charge as described in Section 3.2, this mathematical construction does not rely on an assumed recombination rate. This makes it well-suited for measuring energies of extended objects like showers where the $d E / d x$ (and thus recombination) is difficult or impossible to measure for many of the reconstructed depositions. 


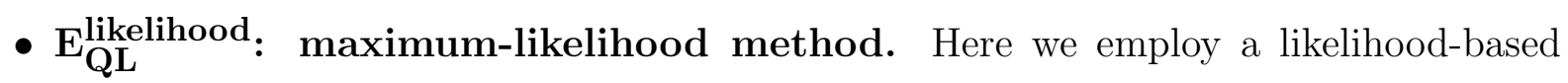
hypothesis testing framework which returns the most probable deposited energy that would result in a particular measured combination of $Q$ and $L$. We use a simplified model where each Michel electron event can be represented by just three parameters: energy $(E)$, reconstructed charge $(Q)$, and reconstructed light $(L)$. This method requires knowledge of the detector's response, averaged over all spatial positions, as a function of energy deposited by the electron shower. We achieve this through the construction of parameterized probability distribution functions (PDFs) which approximate the probabilities of measuring any $Q$ or $L$ given some amount of deposited shower energy in the TPC. This is explained in more detail later.

\subsection{Event selection}

A series of selection cuts are made on the data to ensure only well-reconstructed events contribute to our final physics samples. These cuts can be separated into three categories: optical Michel double-pulse identification, 2D Michel electron shower identification, and 3D shower identification. Here we describe each of the cuts in detail.

In all cases, we try to treat data and MC identically. However, many of the optical data cuts cannot be applied analogously to MC due to the lack of simulated PMT waveforms. Extraction and reconstruction of the optical data values in $\mathrm{MC}$ is thus done in an idealized way that doesn't employ waveform corrections, hit-finding, or the integration techniques discussed in the previous section. Instead, simulated photoelectron arrival time distributions are used as proxy-waveforms and light yields are taken as the number of counts within the same integration regions applied to data (with smearing applied) [75].

Prior to reconstruction-based cuts, events are first filtered based on the average wire signal RMS on the collection plane to exclude unusually noisy events (removes $\approx 2 \%$ of events). Events passing both the cosmic timestamp and wire noise filters are then fed into a standard reconstruction chain. First wire signals are deconvolved and Gaussian-based hit- 
finding is performed using GausHitFinder. The remaining reconstruction steps are skipped if either plane has more than 300 hits reconstructed, as this is usually a sign of high noise or a non-Michel event (removes a negligible number of events, $<0.01 \%$ ).

Optical Michel identification: The optical data plays an essential role in this analysis. The PMT signals are used both in the physical trigger described in Section 4.7 and again in the data analysis to identify Michel-like topologies, reconstruct detected photoelectron yields, and measure muon decay time. Michel electron candidates must pass a series of cuts involving the optical data:

- Waveform RMS cut: The baseline region of the PMTs' waveforms (first $1 \mu \mathrm{s}$ ) must have a signal RMS that does not exceed some threshold. This excludes unusually noisy events from our sample.

- 2 optical hits: Require exactly two optical hits reconstructed in each PMT, giving us a separate $\Delta T_{i}$ for each.

- $\Delta T$ matching: The $\Delta T_{i}$ from each PMT must not differ by more than 15 ns.

- Hit width cuts: It was realized that the full-width-half-max (FWHM) of optical hits could be used to exclude a sizeable fraction of unknown background events that contaminate our data samples. Both the muon and electron hit are required to have a FWHM that exceeds 10 ns.

- 2nd pulse saturation: The candidate Michel pulse cannot saturate the full scale of the digitizer.

- Decay time cut: The measured $\Delta T$ must be greater than some minimum and less than $7200 \mathrm{~ns}$ (the approximate length of the coincidence gate plus its delay in the hardware trigger). The cut requiring a minimum $\Delta T$ is particularly important to avoid efficiency effects that occur at small $\Delta T$ near the boundary of the gate delay period. For this 
analysis, the gate delay was set to about $300 \mathrm{~ns}$ in the trigger, so a $\Delta T_{\min }=350 \mathrm{~ns}$ is used.

2D shower identification: Several quality cuts are made on the 2D Michel shower reconstructed from the collection plane, if one was found.

- Cluster size cuts: $N_{\mu} \geq 8, N_{e} \geq 4$

- Muon linearity cuts: The average local linearity of all the hits in the muon portion of the cluster must exceed 0.7 .

- At least 5 linear muon hits must be used to define its terminal direction vector.

- The 2D decay angle must fall within a defined range, $15^{\circ}<\theta_{2 D}<165^{\circ}$. This cut removes events where the electron track/shower is co-linear with the muon.

\section{$3 D$ shower identification}

- Michel electron showers must be reconstructed in both planes. We do not impose the same level of quality cuts to the induction plane shower as we did for the collection plane shower, since we do not intend to extract a charge-energy measurement from the induction plane. We only need some number of hits to construct spacepoints.

- Minimum 3D points: After hit-matching, the number of 3D points constructed $\left(N_{p t s}^{3 D}\right)$ must be at least 3 .

- The charge-weighted 3D centroid location of the shower must be within a fiducial volume defined by a $4 \mathrm{~cm}$ boundary at the edges of the TPC.

After the above cuts, we impose a final decay-time cut, $\Delta T>1.8 \mu \mathrm{s}$, to ensure the reconstructed light in our sample of events is minimally affected by late-light contamination from the muon pulse. In the optical reconstruction, $1.8 \mu$ s also marks the threshold beyond 
which the binned muon late-light correction procedure is applied, so by making this cut we avoid introducing systematic uncertainties associated with having a mix of events that were reconstructed differently. We must note here that this cut on $\Delta T$ is only imposed to ensure that good-quality reconstruction of $L$ is possible. The data excluded by this cut are not necessarily a "background" - they are still perfectly fine Michel electron events in the TPC, and can thus be reconstructed to extract $Q$ if one wished to do so.

The cut-by-cut event reduction is represented in Tabel 8.1. The fraction of events removed by each cut is displayed as well. Note that, as mentioned in the text, the events included in "total events" are actually a mix of all the different cosmic triggers.

\subsection{Reconstructing charge and light}

The $Q$ for a topology containing $N$ reconstructed wire hits is simply the summation of all individual charge deposits $\left(q_{i}\right)$, each corrected for its drift attenuation based on its drift time $\left(t_{i}\right)$

$$
Q=\sum_{i}^{N}\left(q_{i}^{\mathrm{ADC}} \times \exp \left(t_{i} / \tau_{e}\right)\right) \times C_{e^{-}}^{\mathrm{cal}}
$$

where $q_{i}^{\mathrm{ADC}}$ is the integrated charge in ADC units of each individual hit, while $C_{e^{-}}^{\mathrm{cal}}$ is the ADC-to-electron calibration constant determined in Chapter 6.

Similarly, we calculate $L$ by summing the detected light $S$ from both PMTs and applying quenching corrections and scaling to account for losses during propagation,

$$
L=\sum_{j}^{N^{P M T s}}\left[S^{\mathrm{ADC}} \times C_{p e}^{\mathrm{cal}} \times f_{Q E}^{-1} \times f_{\text {vis }}^{-1} \times C_{\text {quench }}\right]_{j} .
$$

The term $S^{\mathrm{ADC}}$ is the total integrated charge of the candidate pulse on PMT $j$ in ADC units, after muon late-light contamination has been estimated and removed (see Section 5.4.6. The calibration constant $C_{p e}^{c a l}$ is the inverse of the PMT's SPE response determined in Chapter 6. The factor $f_{Q E}$ folds in the PMT's quantum and collection effi- 
Table 8.1: Event reduction table for all data and MC samples.

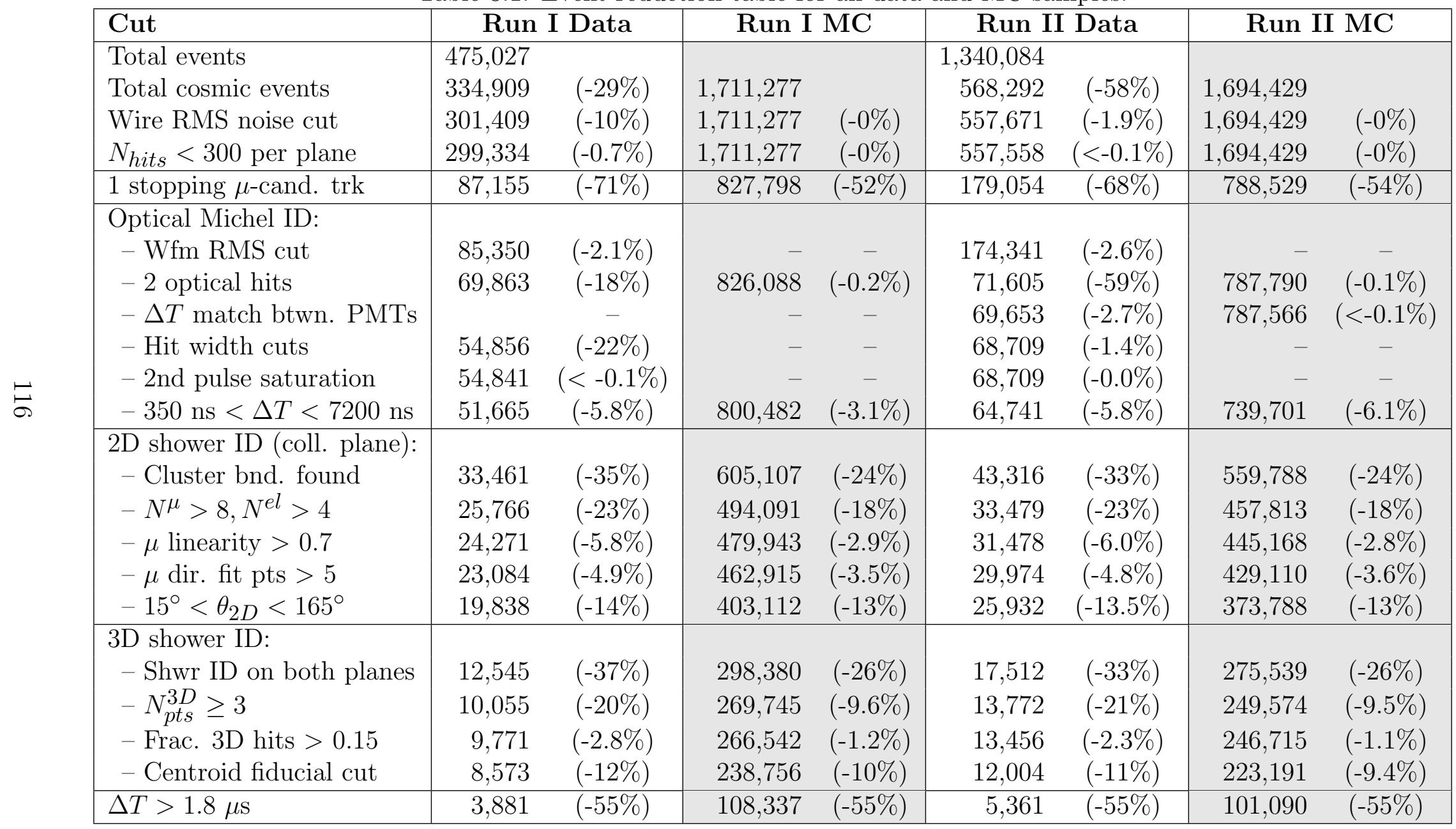




\section{Run I}
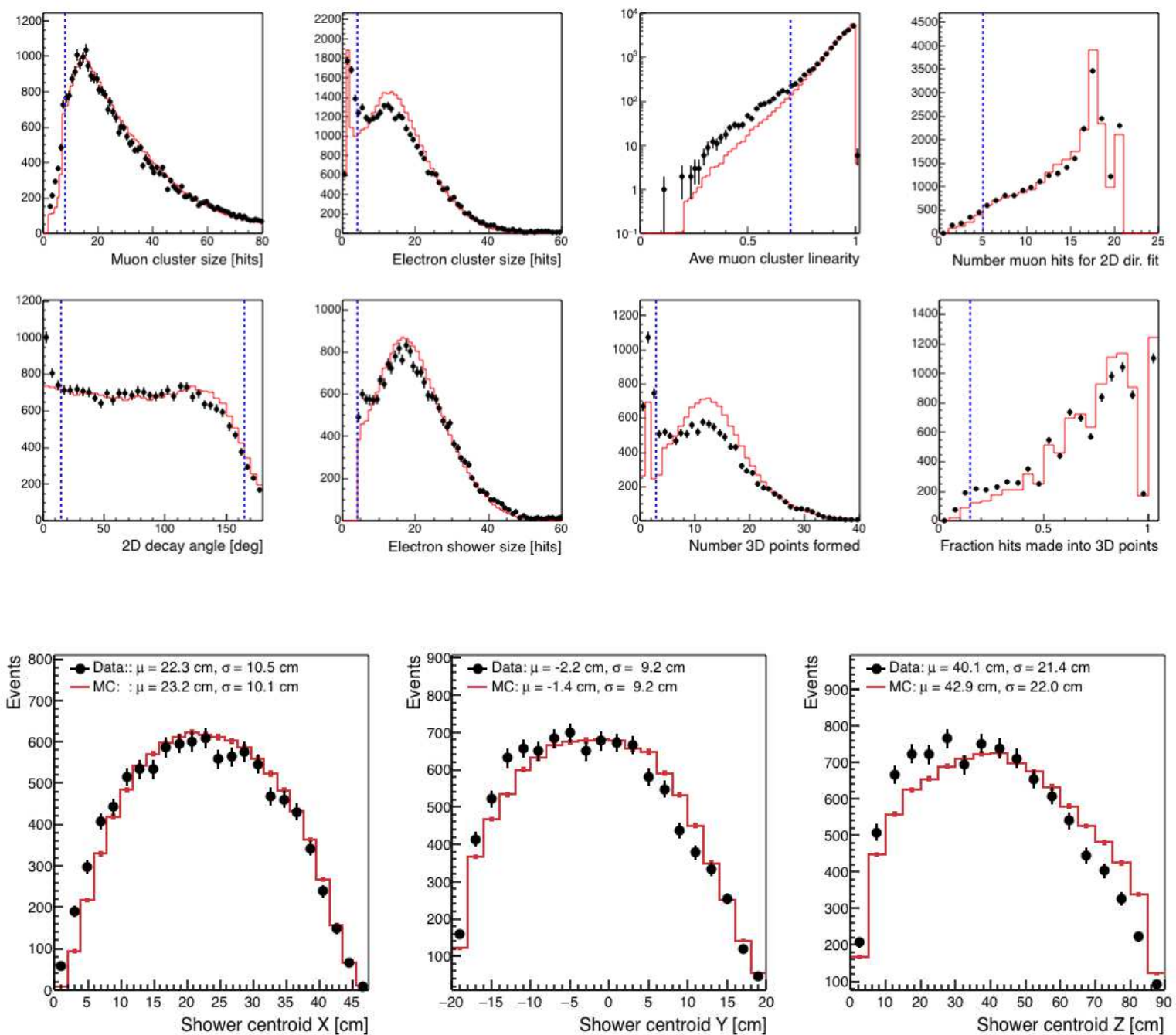

Figure 8.1: Run I event cut distributions (top) and distribution of charge-weighted shower centroids (bottom). Selection boundaries indicated by dashed lines. 


\section{Run IIB}
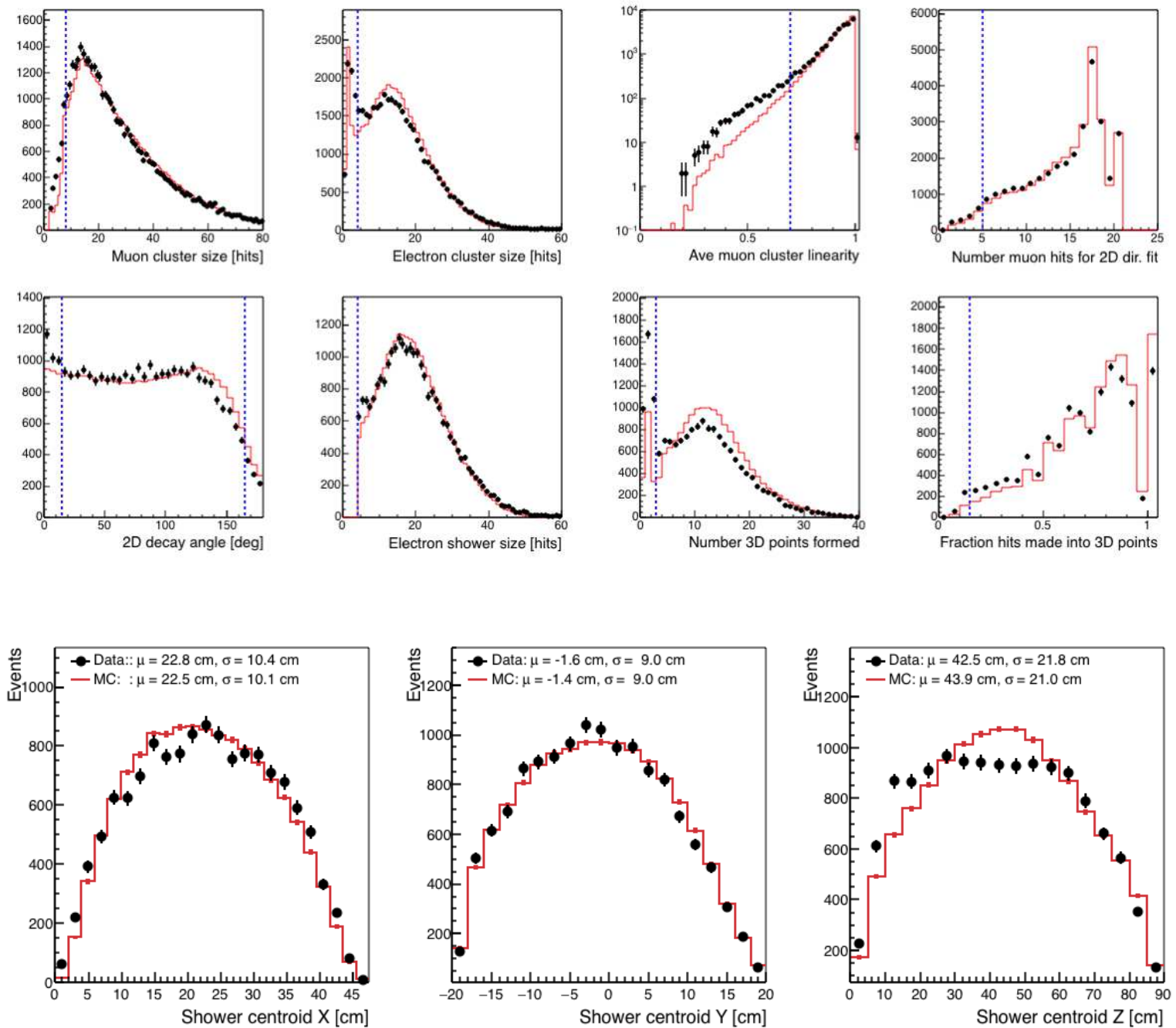

Figure 8.2: Run I event cut distributions (top) and distribution of charge-weighted shower centroids (bottom). Selection boundaries indicated by dashed lines. 

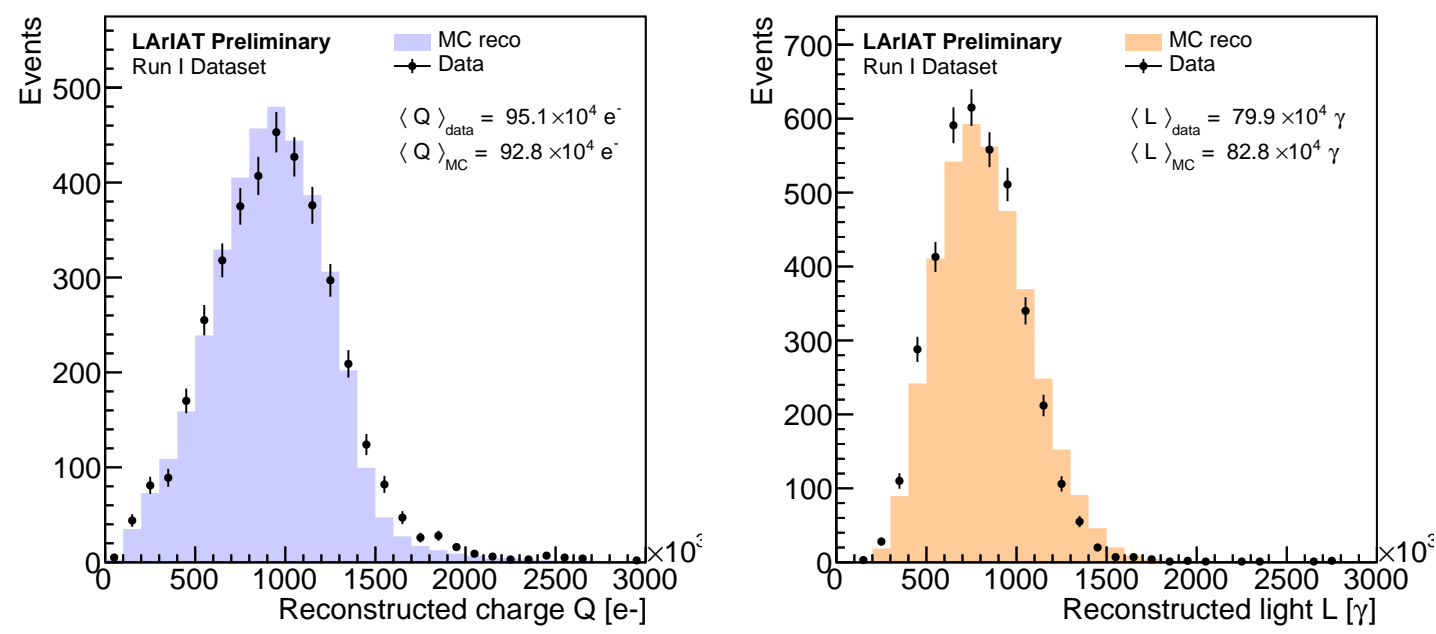

Figure 8.3: Reconstructed distributions of free ionization electrons $(Q)$ and scintillation photons $(L)$ for the Run I Michel electron sample.
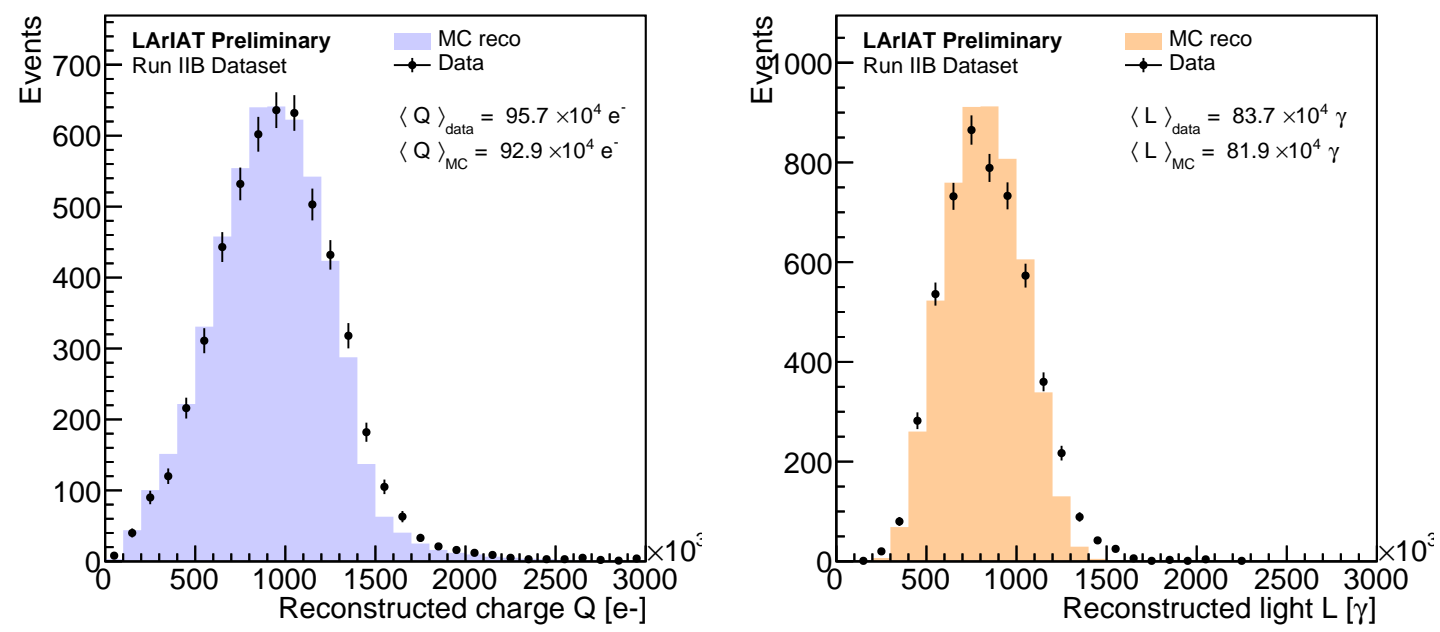

Figure 8.4: Reconstructed distributions of free ionization electrons $(Q)$ and scintillation photons $(L)$ for the Run IIB Michel electron sample. 
ciencies [73]. The visibility and quenching corrections in Equation 8.4 are analogous to the electron attenuation lifetime corrections applied to get $Q$ in Eq. 8.3 in that we are scaling up the collected light to account for expected losses due to propagation and impurity quenching.

The average photon visibility $f_{\text {vis }}$ is calculated as the charge-weighted average of the visibility of each reconstructed 3D space point. For PMT $j$,

$$
f_{v i s}=\sum_{i}^{N^{3 D}}\left[q_{i} \times f_{j}(x, y, z)\right] \times\left(\sum_{i}^{N^{3 D}} q_{i}\right)^{-1}
$$

Here, $f_{j}(x, y, z)$ is the fraction of scintillation photons emanating from the location of each space point expected to reach the window of $\operatorname{PMT} j$ according to the $3 \mathrm{D}$ visibility map.

Since the quenching of prompt light from singlet excimer decays is negligible, the correction factor $C_{\text {quench }}$ is constructed such that only the late component of the total light is scaled up. We define prompt light simply as the light arriving prior to some time $T_{\mathrm{pr}}$ (nominally $100 \mathrm{~ns}$ for this analysis). The quenching correction factor is thus redefined as a function of the prompt fraction $\left(f_{\text {prompt }}\right)$ - i.e., the ratio of the prompt to the total light integral - as well as the triplet excimer lifetime $\tau_{t}$ and the effective quenched late lifetime $\tau_{t}^{\prime}$ found from fits to data,

$$
C_{\text {quench }}=f_{\text {prompt }}+\left(1-f_{\text {prompt }}\right) \times C_{\text {quench }}^{\text {late }}
$$

where the last term is the correction to the late light component:

$$
C_{\text {quench }}^{\text {late }}=\left(\tau_{t} / \tau^{\prime}\right) \exp \left[-T_{\operatorname{pr}}\left(\tau_{t}-\tau_{t}^{\prime}\right)\right]
$$

The distributions of both $Q$ and $L$ for our sample are presented in Figures 8.3 and 8.4 . 

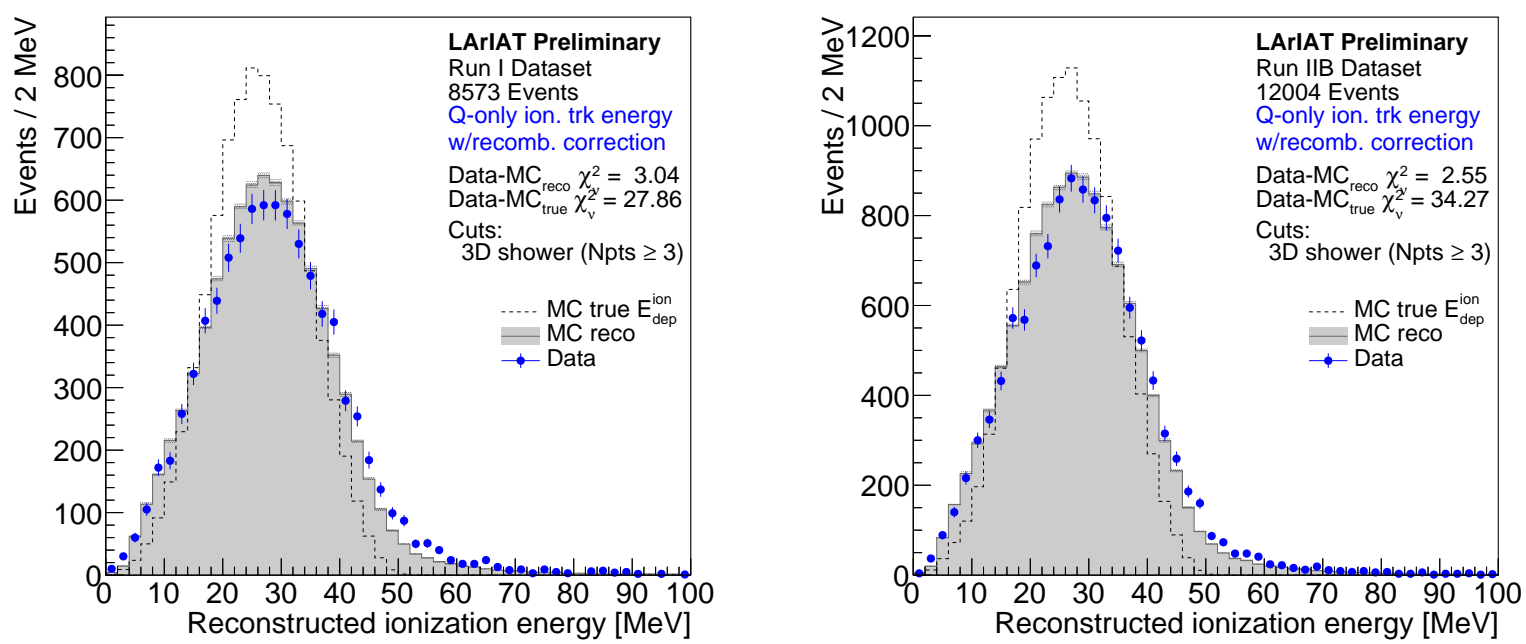

Figure 8.5: Reconstructed energy $E_{Q}^{\text {ion }}$ deposited by the portion of the event identified as the direct electron-induced ionization for Run I (left) and Run II (right).

\subsection{Electron energy spectra using charge and light}

Here we present reconstructed energy spectra for Michel electrons in LArIAT using Equation 8.1 for charge-based energy and Equation 8.2 for the combination of charge and light.

First, we reconstruct charge-based energy $E_{Q}^{\text {ion }}$ from ionization deposited directly by the Michel electron itself, occasionally referred to as the electron ionization track energy. These deposits are defined as hits that were included in the original electron cluster, excluding the addition of photon-initiated deposits within the 2D shower acceptance cone. These spectra are shown in Figure 8.5 .

The reconstructed energy of the full Michel electron shower, including both the electron ionization as well as charge induced by any bremmstrahhlung photons emanating from the electron, are displayed in Figure 8.6. We assume a uniform recombination survival probability of $R=0.69$ for both electron- and photon-initiated charge depositions ${ }^{1}$ In Figure 8.6 we see

1. In actuality the photon-initiated deposits (whether induced through pair-production or Compton scattering) are likely to have a higher $d E / d x$ and thus a smaller fraction of surviving electrons. Monte Carlo suggests that photon deposits vary considerably in their recombination, with $R \approx 0.59$ on average. Attempts to use different recombination factors for electron and photon depositions did not result in significant changes in the resulting energy spectrum. 
Table 8.2: Average light yield (LY) in units of photoelectrons per $\mathrm{MeV}$ (pe/MeV).

\begin{tabular}{|l|c|c|}
\hline Method & Run I & Run IIB \\
\hline Truth (no trigger/fiducial cuts) & $3.7 \mathrm{pe} / \mathrm{MeV}$ & $18.3 \mathrm{pe} / \mathrm{MeV}$ \\
Reconstructed quantities in data & $4.3 \mathrm{pe} / \mathrm{MeV}$ & $17.6 \mathrm{pe} / \mathrm{meV}$ \\
Reconstructed quantities in MC & $4.3 \mathrm{pe} / \mathrm{MeV}$ & $18.3 \mathrm{pe} / \mathrm{MeV}$ \\
\hline
\end{tabular}

a tail extending to lower energies in $E_{Q}$ which is mitigated by the addition of light in $E_{Q L}$. This underscores the power of $E_{Q L}$ - information that would be lost using only charge (either due to higher-than-assumed recombination, incomplete clustering, or hit thresholding) is recovered to some extent in the optical data.

We can now use this information to estimate LArIAT's light yield, or number of photoelectrons detected per unit energy deposited. The light yield can be measured either from reconstructed data or, given the good agreement between data and MC, taken directly from truth-level information in the simulated sample. Results from both methods are summarized in Table 8.2. Fiducial margin cuts applied in the event selection (shower centroid $>4$ cm from TPC walls) will bias the light yield measured from reconstructed quantities to be slightly lower, since the highest fractional photon visibilities occur closer to the TPC boundaries where the wavelength-shifting TPB foils are mounted. On the other hand, the nature of the light-based trigger will bias our selection toward more luminous events. Therefore, the net bias in the measured light yield using reconstructed events is hard to predict. The benefit of using the truth-level information is that it is not influenced by the trigger efficiency and fiducial cuts.

It's worth noting the match to MC in the spectra in Figures 8.5 and 8.6 are fairly consistent between the Run I and Run IIB. However, it is known that Run I had generally poorer data-taking conditions such as higher RMS noise on the wires and the additional noise induced on the wires by the negatively-biased PMT photocathodes. For this reason, along with the fact that $\approx 10$ times more physics data in general (used in other analyses) were collected in Run II than Run I, we will focus only on Run IIB for the remainder of this analysis as we develop a likelihood fitter and assess energy resolution using Monte Carlo. 

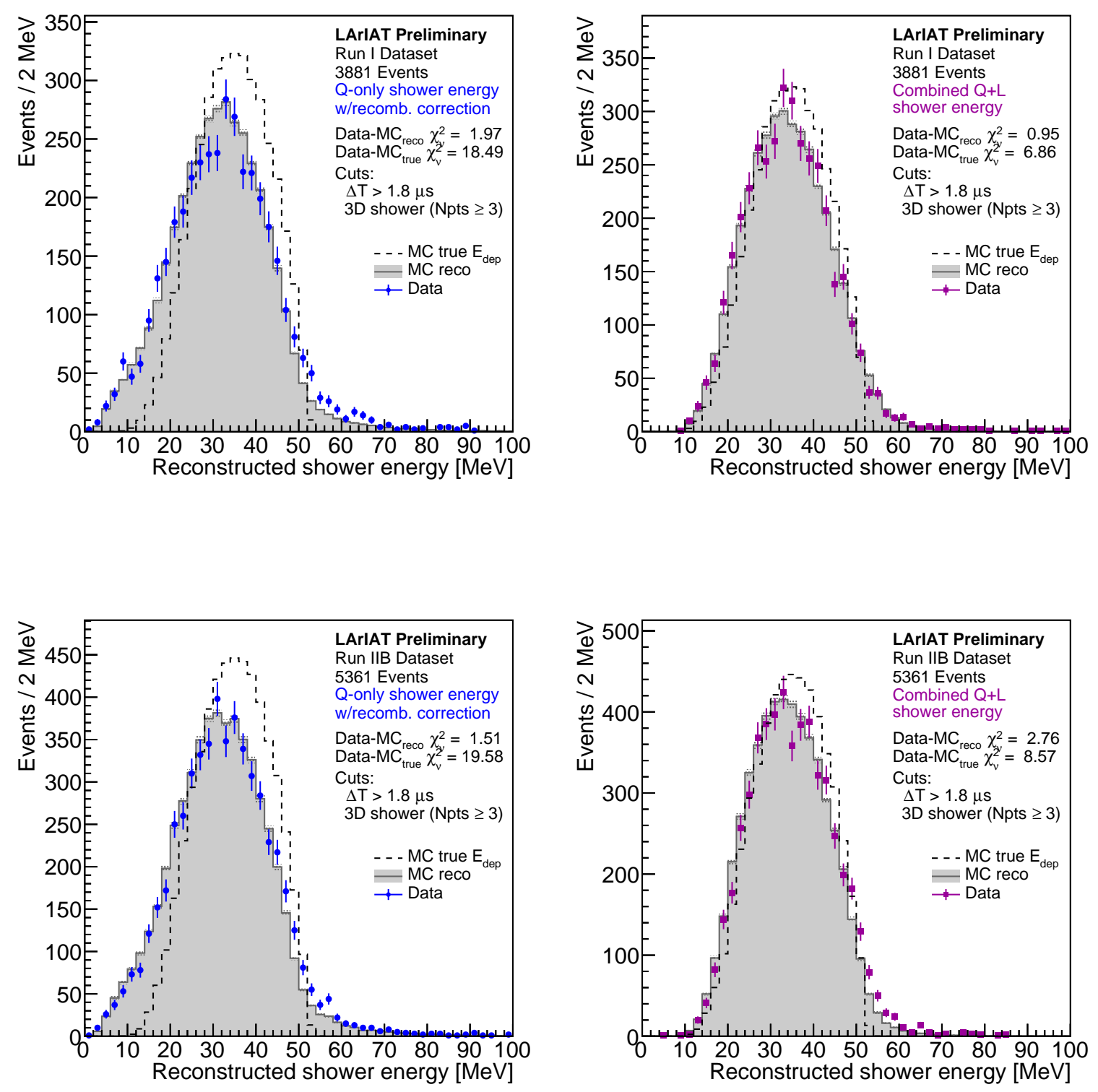

Figure 8.6: Reconstructed energy of the full Michel electron shower for Run I (top) and Run II (bottom). Equation 8.1 is used to calculate the charge-based $E_{Q}$ (left), while Equation 8.2 is used to calculate energy by directly combining charge and light (right). 


\subsection{Maximum likelihood method for $\mathrm{Q}+\mathrm{L}$}

We now turn to assembling a more sophisticated tool for reconstructing Michel electron energy from charge and light. We make use of the maximum-likelihood hypothesis technique which finds the most likely energy that would produce each measured combination of $Q$ and $L$ given the detector's expected performance in reconstructing these two quantities. Much of this section is taken from a paper on this analysis soon to be published [87].

The likelihood of reconstructing a Michel electron event with measured $Q$ and $L$, given a true deposited energy $E$, is given by

$$
\mathcal{L}(Q, L ; E)=f_{Q}(Q ; E) f_{L}(L ; E)
$$

where $f$ denotes the probability distribution function (PDF) for the measured charge or light. For each event we seek to find the $E$ that maximizes $\mathcal{L}$, so we perform a minimization over the negative logarithm:

$$
\begin{aligned}
F(Q, L ; E) & =-\log \mathcal{L}(Q, L ; E) \\
& =-\log f_{Q}(Q ; E)-\log f_{L}(L ; E) .
\end{aligned}
$$

To determine the energy-dependent PDFs, we first use the Monte Carlo sample (with trigger efficiency cuts turned off) to assemble histogrammed distributions of reconstructed charge and light at different values of true deposited shower energy. These "slices" in energy are made at regular intervals of $5 \mathrm{MeV}$ and are relatively narrow $( \pm 0.5 \mathrm{MeV})$ to minimize smearing of the distributions due to contributions from events of widely differing energies. We then find that each $L$ distribution can be fit to a single Gaussian:

$$
f_{L}^{*}(L)=N \exp \left[-\frac{(L-\mu)^{2}}{2 \sigma^{2}}\right] .
$$

However, to fit $Q$, we require two Gaussians - one modeling the central "peak" and another 

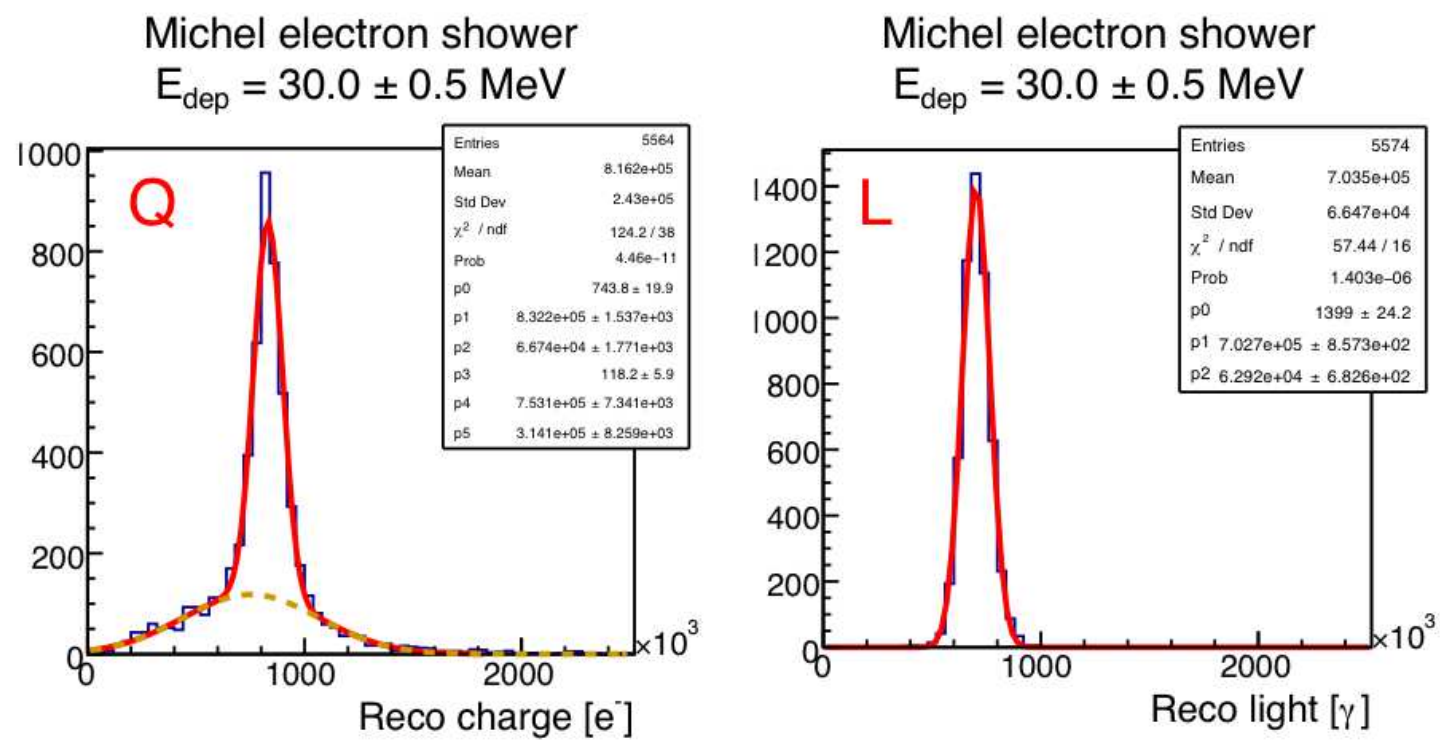

Figure 8.7: Examples of reconstructed $Q$ (left) and $L$ (right) distributions for simulated Michel electrons depositing $30 \mathrm{MeV}$ in the LArIAT TPC. The red lines are fits to the distribution. For $Q$ the fit is to a double-Gaussian function, while $L$ is fit to a single Gaussian at all energies. The dotted orange line in $Q$ illustrates the "background" (BG) Gaussian in the double-Gaussian fit.

that models the more diffuse "background" population of events:

$$
f_{Q}^{*}(Q)=N_{p} \exp \left[-\frac{\left(Q-\mu_{p}\right)^{2}}{2 \sigma_{p}^{2}}\right]+N_{B G} \exp \left[-\frac{\left(Q-\mu_{B G}\right)^{2}}{2 \sigma_{B G}^{2}}\right]
$$

This distinct non-Gaussian distribution in charge is a result of reconstruction effects specific to the Michel electron sample such as charge overlap between the muon and electron and incomplete clustering of the Michel shower. Events with $Q$ values that populate the central peak of these distributions are those that are well-reconstructed. Examples of fitted distributions at $30 \mathrm{MeV}$ for both $Q$ and $L$ are shown in Figure 8.7, with all nine shown in Figure 8.8 and Figure 8.9 .

The functions $f_{Q}^{*}$ and $f_{L}^{*}$ when normalized to unity are equivalent to the $\operatorname{PDFs} f_{Q}(Q ; E)$ and $f_{L}(L ; E)$ needed to compute likelihood $\mathcal{L}$. In order to extrapolate between the disparate energy bins used to make the nine fits and predict $f_{Q}$ and $f_{L}$ for all deposited energies, we model the parameters of each fit as arbitrarily-chosen functions of $E$ over the range of 

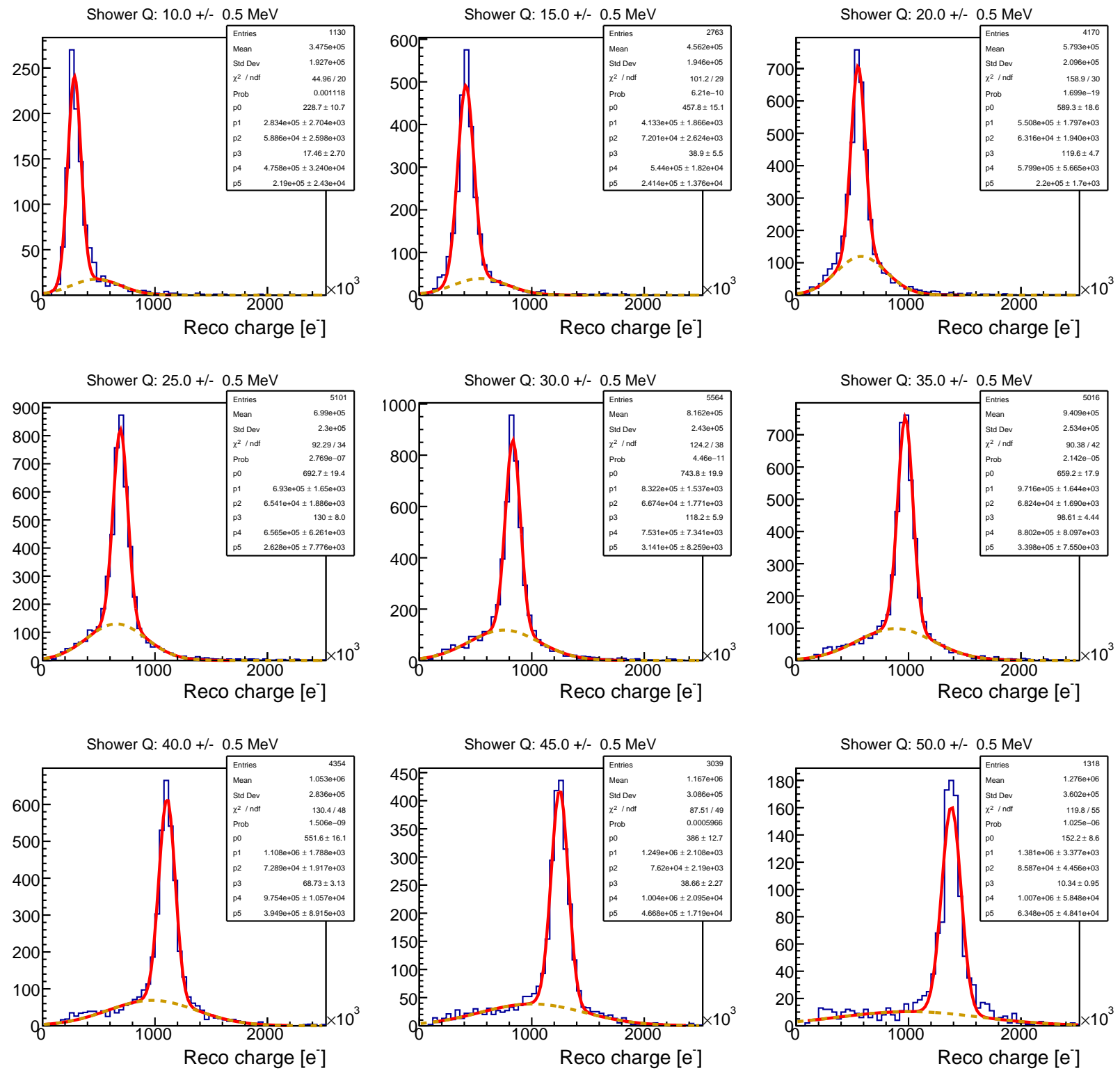

Figure 8.8: Reconstructed $Q$ distributions for events of different deposited energies in the LArIAT TPC. Events are distributed randomly, so each distribution is effectively an average over all possible locations. The red line is a fit to a double-Gaussian function. The dotted orange line illustrates the secondary "background" (BG) Gaussian in the total fit. (Parameters: $\left.N_{\text {peak }}=\mathrm{p} 0, \mu_{\text {peak }}=\mathrm{p} 1, \sigma_{\text {peak }}=\mathrm{p} 2, N_{B G}=\mathrm{p} 3, \mu_{B G}=\mathrm{p} 4, \sigma_{B G}=\mathrm{p} 5.\right)$ 

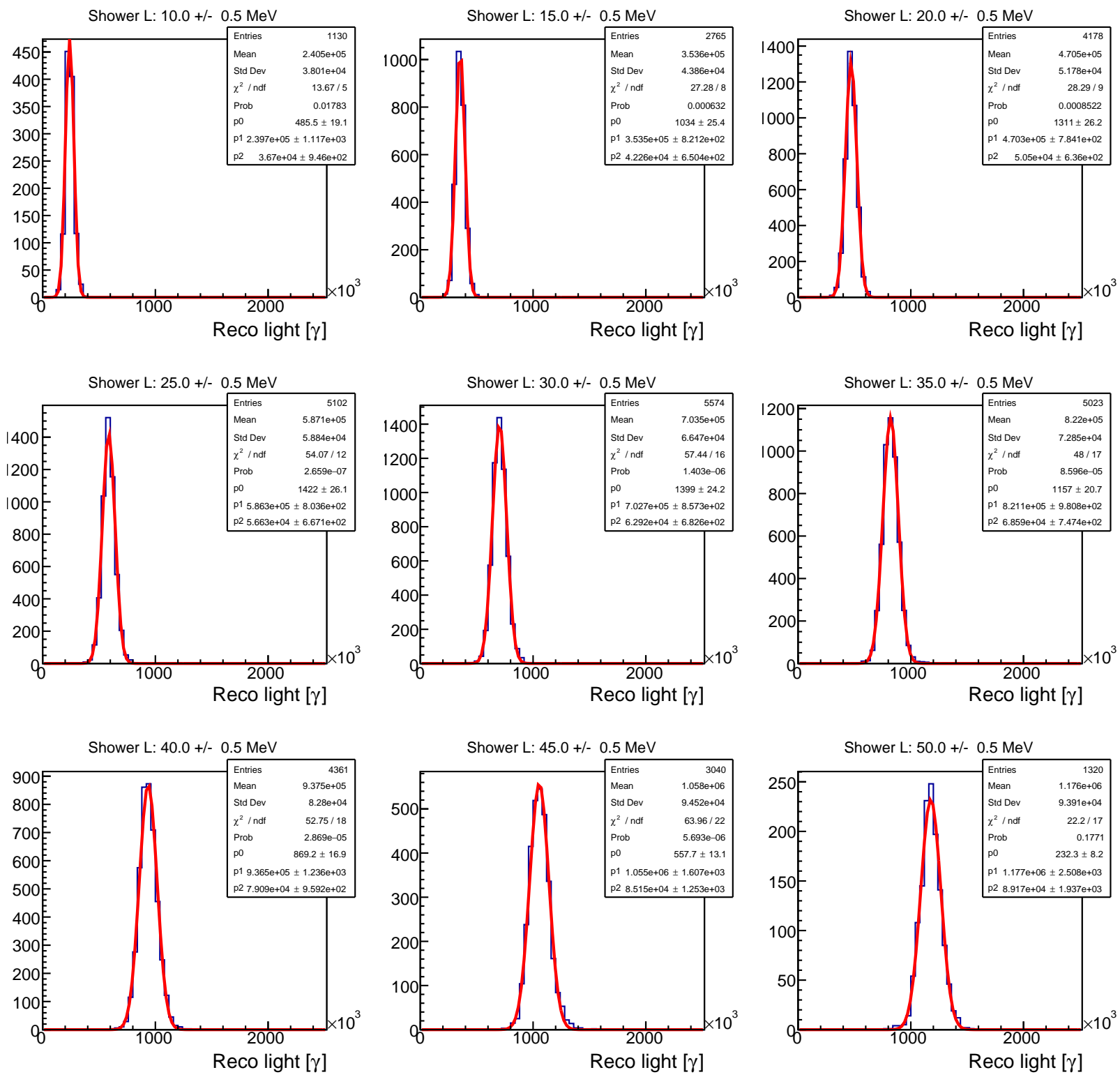

Figure 8.9: Reconstructed $L$ distributions for events of different deposited energies in the LArIAT TPC. Events are distributed randomly, so each distribution is effectively an average over all possible locations. The red line is a fit to a Gaussian function. (Parameters: $N=$ $\mathrm{p} 0, \mu=\mathrm{p} 1, \sigma=\mathrm{p} 2)$ 
relevant deposited energies (5-50 MeV).

For $f_{Q}^{*}$, neglecting the overall normalization (which will be fixed to one), we find that specific combinations of parameters can be modeled as follows:

$$
\begin{aligned}
{\left[\mu_{p}\right]_{Q} } & =p_{0}+p_{1} E \\
{\left[\sigma_{p} / \mu_{p}\right]_{Q} } & =p_{0} / E^{2}+p_{1} / E+p_{2} \\
{\left[A_{B G} / A_{p}\right]_{Q} } & =p_{0}+p_{1} E+p_{2} E^{2}+p_{3} E^{3} \\
{\left[\mu_{B G} / \mu_{p}\right]_{Q} } & =p_{0} / E^{p_{1}}+p_{2} \\
{\left[\sigma_{B G} / \sigma_{p}\right]_{Q} } & =p_{0}+p_{1} E+p_{2} E^{2}
\end{aligned}
$$

The terms $A$ above refers to the integral of the Gaussian component, $A=\sqrt{2 \pi} N \sigma$. Similarly for $f_{L}^{*}$, we are able to reproduce each fit completely with only two parameterizations:

$$
\begin{gathered}
{[\mu]_{L}=p_{0}+p_{1} E} \\
{[\sigma / \mu]_{L}=p_{0} / E^{p_{1}}+p_{2}}
\end{gathered}
$$

These fitted parameterizations are displayed in Figure 8.10. Using these, we are then able to, on average, construct the predicted shape of the distribution of $Q$ and $L$ for events of any deposited energy. This is illustrated by the parameterization overlays in Figure 8.12

With $f_{Q}$ and $f_{L}$ defined as the unity-normalized functions $f_{Q}^{*}$ and $f_{L}^{*}$, we now have all the pieces necessary to construct the event likelihood in Eq. 8.7. Figure 8.13 shows the resulting spectrum of Michel electron shower energy in Run IIB using this likelihood method, compared to the spectra for the mathematical constructions $E_{Q}$ and $E_{Q L}$ presented earlier. We see that the likelihood method performs similarly to $E_{Q L}$, though its match to the true energy deposited is slightly improved. 

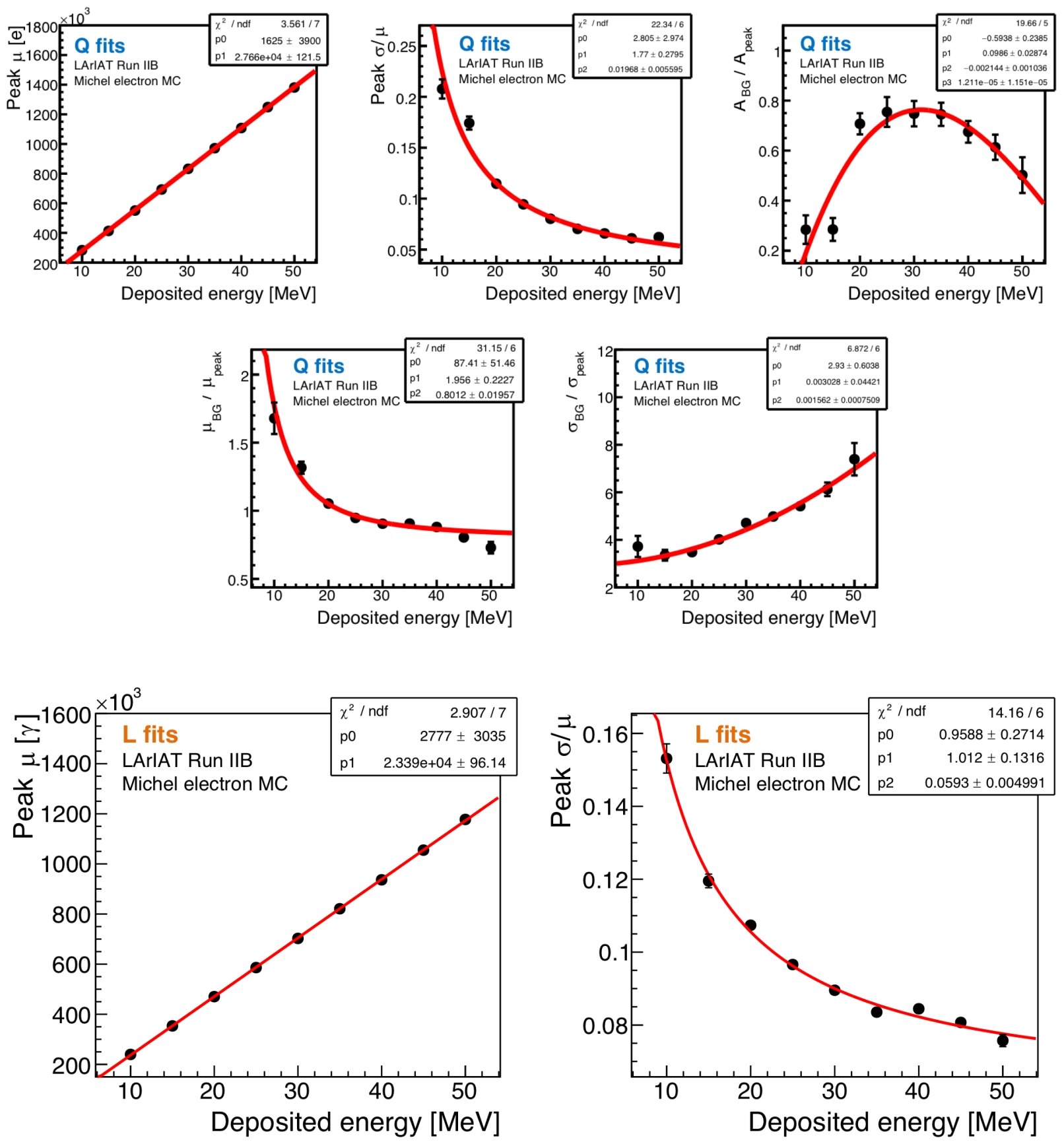

Figure 8.10: Parameters describing the charge $\operatorname{PDF} f_{Q}$ (top) and light $\operatorname{PDF} f_{L}$ (bottom) modeled as functions of energy deposited in the TPC by the Michel electron shower. The equations for the fits to $f_{Q}$ parameters are outlined in Equations 8.10 8.14, while those for $f_{L}$ are in Equations 8.15,8.16. 

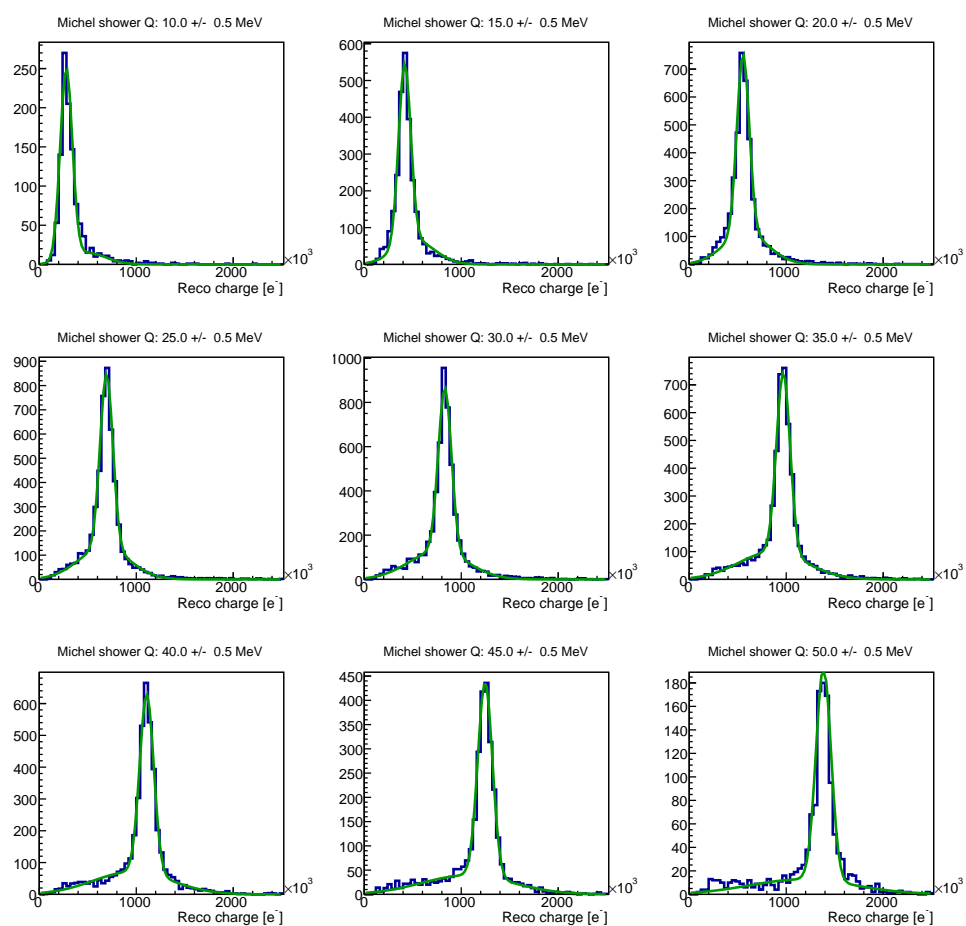

Figure 8.11: Charge distributions overlaid with parameterizations.
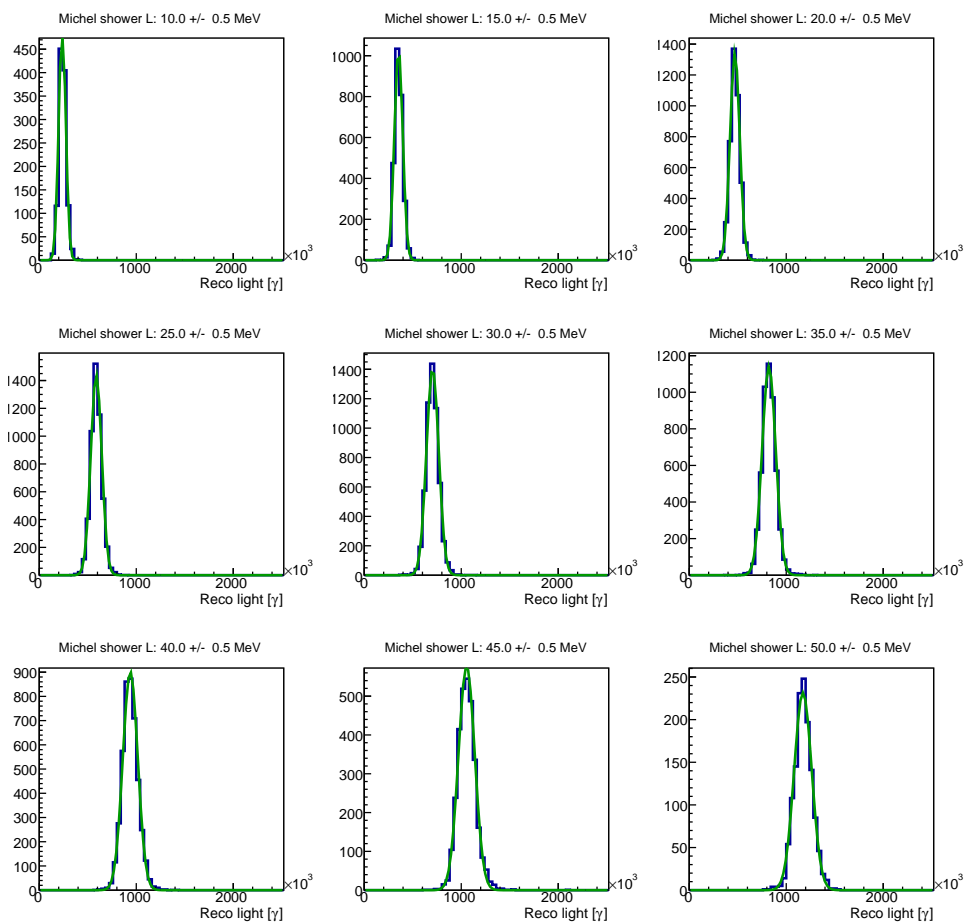

Figure 8.12: Light distributions overlaid with parameterizations. 

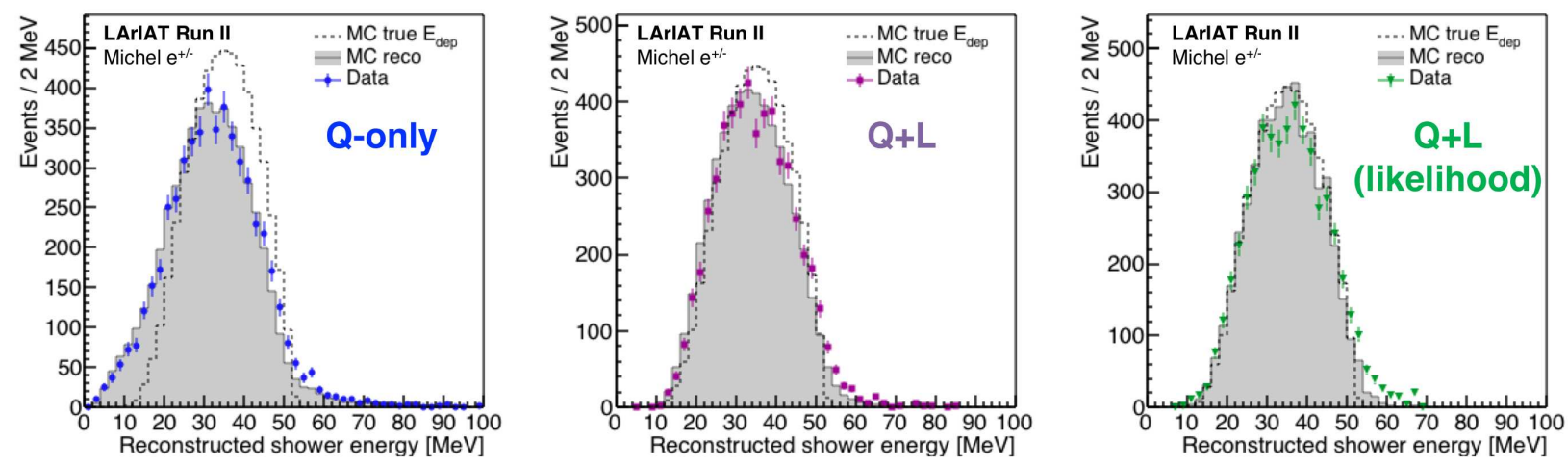

Figure 8.13: A comparison of the Michel electron shower energy spectrum from Run IIB reconstructed using the the two simple formulaic constructions, $E_{Q}$ (left) and $E_{Q L}$ (middle),

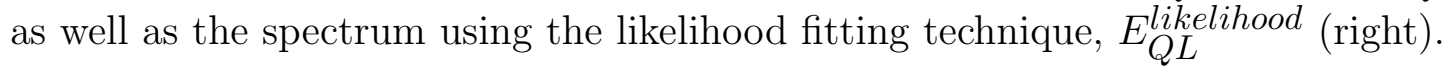

\subsection{Monte Carlo energy resolution}

With our simulations validated by data, we now use MC to compare the energy resolution $(\sigma / E)$ of these three calorimetric techniques. Resolution is a metric for quantifying precision

- but in the analysis that follows, its exact definition is slightly fluid. When characterizing resolution, we first create a histogram of the energy variance, $\delta E$, on an event-by-event basis:

$$
\delta E=\frac{E^{\text {reco }}-E^{\text {true }}}{E^{\text {true }}} .
$$

In an idealized system, $\delta E$ forms a perfect Gaussian and we take the resolution to be its fitted width or standard deviation (RMS). However, when the variance takes on a non-Gaussian shape, as we will see in the Michel electron sample, the resolution becomes ill-defined and the definition must be modified to fit the situation.

First we attempt to quantify deposited energy resolution for Michel electron events in LArIAT's Run II. We then turn to a simulated sample of isolated, fully-contained electron showers distributed randomly within the active volume to determine the calorimetric resolution of this topology free from complications brought about by the Michel electron reconstruction procedure. 


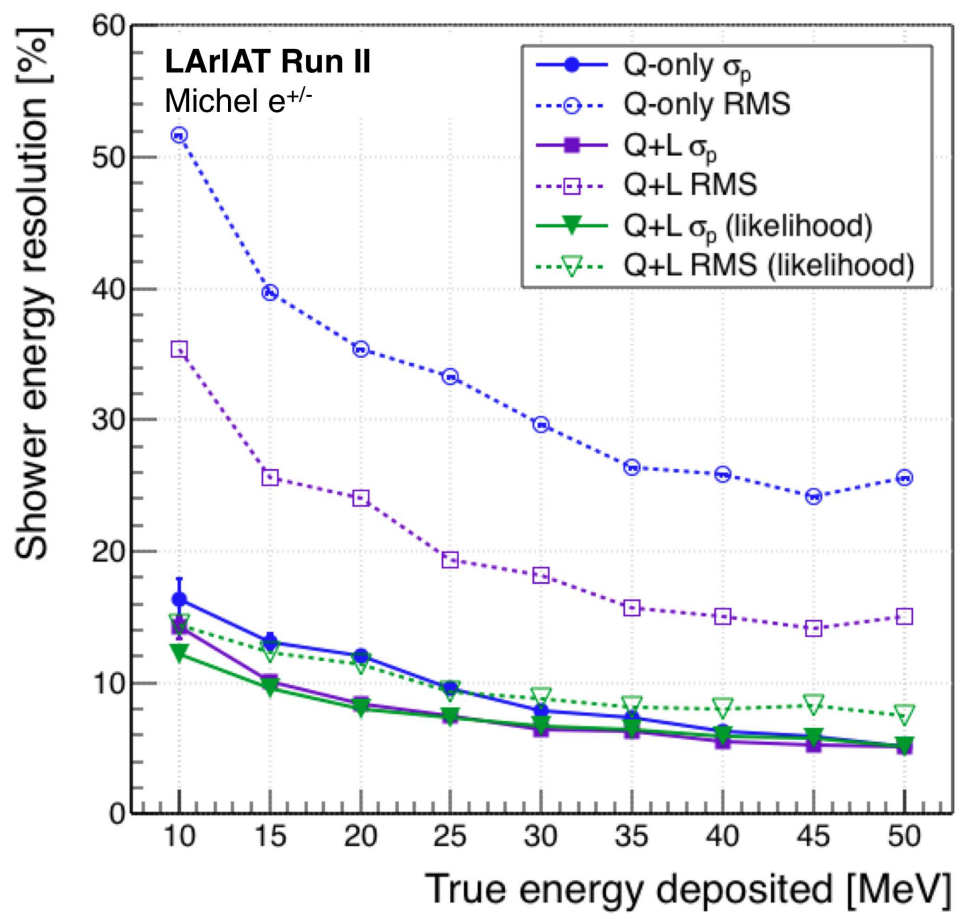

Figure 8.14: The energy resolution of Michel electron events in LArIAT for energies reconstructed from charge (blue) as well as from both charge and light (violet and green).

\subsubsection{Michel electrons}

Energy variance histograms for Michel electron events of different true energy deposition from the Run IIB MC sample are constructed, using the same $0.5 \mathrm{MeV}$ energy "slices" as before but starting at $10 \mathrm{MeV}$ due to statistical limitations of the Michel spectrum at low energy (see Figures B.4 B.5 in Appendix B). Due to the double-Gaussian shape in the distribution of $Q$ for the Michel electrons, these energy resolution distributions take on a similarly nonGaussian shape consisting of a central "peak" region of relatively well-reconstructed events which sits on top of a more diffuse background. In characterizing the resolution, we take into account both the width of the peak as well as the RMS.

To fit the peak in each energy resolution histogram, we define a fit region spanning from the peak (the bin with the highest number of counts) to the points on either side of this peak where the distribution drops to $1 / 3$ the maximum height (a minimum of 8 bins total is used in the fit, \pm 4 surrounding the peak). These fits are presented in Appendix $B$ as 
Figures B.1 B.3. In Figure 8.14, the peak width and RMS is plotted against the deposited energy for each of the three different calorimetric methods for Michel electrons. We see

a drastic improvement in terms of the RMS resolution when using $E_{Q L}$ and $E_{Q L}^{\text {likelihood }}$ compared to the traditional charge-based $E_{Q}$, a consequence of information being recovered from events where some portion of the charge is not reconstructed. In the peak region, the $Q+L$ energies slightly outperform the $Q$-only energy. The likelihood technique performs comparably to $E_{Q L}$ in terms of the peak width.

\subsubsection{Isolated electrons}

The Michel electron reconstruction is vulnerable to inaccurate muon-electron boundary determination, muon-electron charge overlap, incomplete shower clustering, and optical contamination from the late light of the muon. Considering these complications, the sample acts as a poor representation of the low-energy electron showers that would be induced by supernova or solar neutrinos in a deep underground LArTPC. To better study this simpler topology, we reconstruct a simulated sample of lone electrons $\left(e^{-}\right)$positioned randomly in the LArIAT active volume.

The procedure for constructing parametrizable models of $Q$ and $L$ is repeated. We now require that the electron and all of its shower products remain fully contained within the TPC, ensuring that all of the electron's energy is deposited as visible energy in our active volume. Therefore, we now use the initial energy of the electron $\left(E_{e}^{\text {true }}\right)$ as our "true" energy instead of only the visible energy as we did for the Michel electrons (for which no containment requirement was applied). Unlike with the Michel electrons, where reconstruction effects distorted $f_{Q}$ into a non-Gaussian shape, both $Q$ and $L$ distributions for these isolated showers are well-described by a single Gaussian function at each energy. The parameters of $f_{Q}$ and $f_{L}$ are modeled as fitted functions of $E_{e}^{\text {true }}$ with the same equations used to model $f_{L}$ previously for the Michels. See Figures B.4 B.5 in Appendix B for a full array of these fits. 


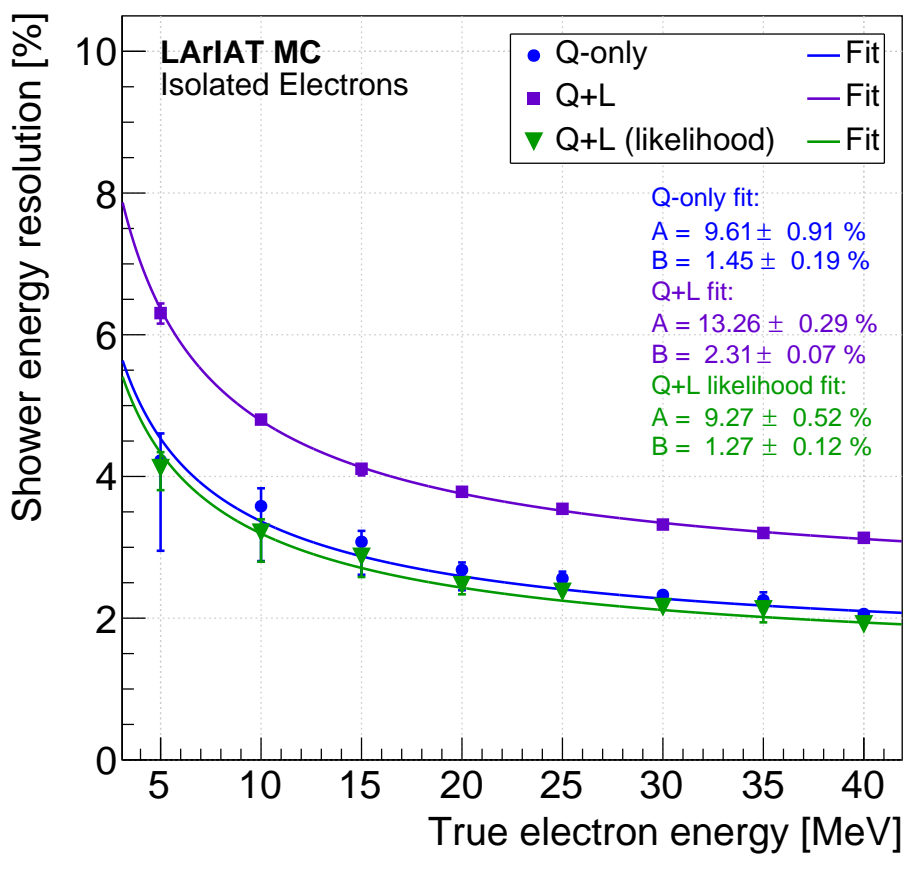

Figure 8.15: The energy resolution, as defined by Equation 8.18, of isolated electron showers in the LArIAT TPC under Run II operating conditions.

We now compare the energy resolution of all three calorimetry methods for this sample of electron showers. To account for any deviations from Gaussian shapes in these distributions, we incorporate both the width of a fitted Gaussian $\left(\sigma_{\text {fit }}\right)$ as well as the RMS $\left(\sigma_{\mathrm{RMS}}\right)$ of the distribution in our definition of energy resolution by assigning a relative weight $(w)$ based on the overall goodness-of-fit:

$$
\sigma=\frac{\sigma_{\mathrm{fit}}+w \sigma_{\mathrm{RMS}}}{1+w}
$$

where $w=\sqrt{\chi_{\nu}^{2}}$. With this definition, the resolution from variance distributions that stray from a Gaussian shape $\left(\chi_{\nu}^{2}>1\right)$ will tend to more heavily weight the RMS rather than the Gaussian fit. On the other hand, well-behaved distributions $\left(\chi_{\nu}^{2} \approx 1\right)$ will weight both the fit and the RMS equally and the assigned resolution will be approximately the average of the two.

The resulting energy resolution curves for isolated electrons in LArIAT using the three 
calorimetric methods are plotted in Figure 8.15. Each set of points is fit to the function:

$$
\sigma(E)=\frac{A[\%]}{\sqrt{E[\mathrm{MeV}]}} \oplus B[\%]
$$

where the first term $(A)$ is meant to model any noise dependence while the second term accounts for reconstruction-specific effects related to hit fitting and thresholding that limit the achievable resolution [55]. Since the resolution in the reconstructed charge now exceeds that of light due to the simpler charge topology, $E_{Q}$ outperforms $E_{Q L}$. In other words, adding $Q$ and $L$ together results in a poorer final resolution than what one would achieve from simply scaling up the charge based on the assumed recombination, $Q \times R^{-1}$. However, as expected, the best resolution is achieved through the likelihood method which naturally takes into account the poorer resolution of $L$ through the PDFs which are constructed from MC. Since $f_{Q}(Q ; E)$ is narrower than $f_{L}(L ; E)$ at all energies, charge is now "weighted" more strongly than light in the determination of $E_{Q L}^{\text {likelihood. }}$.

For low-energy electrons from 5-50 MeV, we find that LArIAT achieves an energy resolution characterized as:

$$
\begin{aligned}
\text { Charge-only: } \quad \sigma & =\frac{9.6 \%}{\sqrt{E}} \oplus 1.5 \% \\
\text { Charge and light: } \quad \sigma & =\frac{9.3 \%}{\sqrt{E}} \oplus 1.3 \%
\end{aligned}
$$

\subsection{Discussion of systematic uncertainties}

Outlined below are a list of effects specific to charge, light, and optical hit timing that are expected to introduce systematic errors to the results presented so far in this thesis.

\section{Charge-based systematics}

- Drift attenuation: $\tau_{e}$ is used to correct for the attenuation of drifted electrons collected on the wires and recover the total free charge produced by the ionization. Calibration 
values are calculated for semi-regular time periods over the course of LArIAT's datataking and an average $\tau_{e}$ is found for each of these periods and saved to a database. Smearing of reconstructed charge (and thus energy) can be introduced if this timebased binning is too coarse relative to the true change in $\tau_{e}$ over time. For the Run IIB Michel electron dataset, the measurement of $\tau_{e}$ had an uncertainty of $\approx 6 \%$ from the fit for each sub-period, each of which was roughly one day long (depending on the number of through-going cosmics available in that period to make the measurement as described in Chapter 6). For an average hit drift time of $\approx 150 \mu$ s and an average lifetime $\left\langle\tau_{e}\right\rangle=800 \mu \mathrm{s}$, this translates to an uncertainty in the collected charge of about $\pm 1.4 \%$.

- Calorimetry conversion constants: Any offset or uncertainty in $C_{e}^{c a l}$, the value used to convert $\mathrm{ADC} \rightarrow e^{-}$), would manifest as a linear shift in charge and energy. As shown in Chapter 6 these calibration constants are measured to good precision $(\lesssim 0.5 \%)$.

- Recombination factor: As discussed, an average recombination scaling factor is determined from MC for use in scaling up charge deposits in reconstructed Michel showers. While a uniform value is used for all Michel electron hits, there is in actuality some variation expected in the recombination rate. This contributes some smearing of the reconstructed charge, which propagates to smearing in the energy.

- Angular effects: Tracks that deviate too far from $90^{\circ}$ in angle relative to the electric field can suffer from calorimetric complications due to extended overlap of drifting electron and ion clouds [58].

- Misreconstruction of Michel showers: It's possible for the hit corresponding to the actual muon endpoint to be misidentified during the clustering stage. If the endpoint is identified too early in the cluster, Bragg-peaked hits of the muon will be clustered in with the electron, adding to its energy. Alternatively, an identified boundary that 
overshoots the true muon endpoint will miss some of the energy deposited by the electron.

\section{Light-based systematics}

- Effects of waveform cleanup techniques: The waveform smoothing, overshoot corrections, and running baseline subtraction may suppress or add to the total detected light. These procedures may also alter the measured muon late-light lifetimes from data, which are used in late-light corrections and as input to the MC.

- Muon late-light corrections: We know from MC that even in ideal circumstances, neglecting noise fluctuations, muon late-light correction described in Section 5.4.6 is imperfect and fails to fully remove all of the muon light that contaminates the Michel electron pulse integration region. This is due in part to the complications introduced by the TPB reemission, which has time constants as long as $3.5 \mu \mathrm{s}$. This causes us to slightly overestimate detected light yields.

- SPE response accuracy: Any error in the single photoelectron (SPE) response measured on the PMTs will result in an overall linear scaling of light measured in data. Also, much like the electron lifetime, if SPE response values are not binned with enough granularity in time, they can introduce a smearing in the detected light integrals if the true gain of the PMT happens to change more rapidly. For Run IIB, the SPE responses are measured to very high precision $(\lesssim 0.3 \%)$, though the value is only measured for 3 -day-long increments, varying by at most $\approx 2 \%$ between subsequent sub-periods.

- Approximations used in photon simulation: There were many broad approximations made in the photon propagation simulation described in Section 7.1. An inaccurate visibility map, either in overall scale or spatial distribution, will lead to inaccurate simulated light distributions as well as cause distortions in the reconstructed $L$, since the $3 \mathrm{D}$ visibility information from these maps is used as one of the necessary corrections 
Table 8.3: The largest sources of systematic error on calorimetric reconstruction.

\begin{tabular}{|l|c|l|}
\hline Variable & $\begin{array}{c}\text { Estimated Impact } \\
\text { on Energy }[ \pm \mathbf{\%}]\end{array}$ & Affected E \\
\hline Electron lifetime $\tau_{e}$ & 1.4 & $E_{Q}, E_{Q L}$ \\
\hline SPE response & 2.0 & $E_{Q L}$ \\
\hline PMT detection efficiency & 2.5 & $E_{Q L}$ \\
\hline$W_{\text {ion }}$ & 1.3 & $E_{Q}$ \\
\hline$W_{\mathrm{ph}}$ & 5.0 & $E_{Q L}$ \\
\hline
\end{tabular}

in converting $S$ into $L$. The collection efficiencies of the PMTs are quoted as $75-80 \%$ for the ETL PMT and 90-95\% for the HMM PMT, so we can conservatively estimate an overall uncertainty in the detection efficiency of $\pm 2.5 \%$.

- Incomplete simulation of PMT response: Without a full electronics response simulation for the PMTs, we must perform a somewhat "idealized" optical reconstruction in the MC that neglects effects from electronic noise, PMT overshoot, as well as any residual systematic effects introduced by waveform processing methods discussed in the first bullet above. We must assume that the artificial smearing of the optical quantities in MC accounts for these effects.

In addition to these, uncertainties in $W_{\text {ion }}(=23.6 \pm 0.3 \mathrm{eV})$ and $W_{\mathrm{ph}}(=19.5 \pm 1.0 \mathrm{eV})$ are propagated to the final calculated energies. Among all of these, the largest quantifiable sources of systematic uncertainty are summarized in Table 8.3. The total propagated error is $\approx 2 \%$ on $E_{Q}$ and $\approx 5 \%$ on $E_{Q L}$. These uncertainties are not expected to change the overall conclusions of our study which are based on relative calorimetric improvements. 


\section{9}

\section{Calorimetric Potential in Future LArTPCs}

Here we expand the MC resolution studies for isolated low-energy electron showers in order to determine the relative impact of light-augmented calorimetry for larger LArTPCs with different wire noise levels and light yields (LYs). For example, DUNE's baseline design anticipates a signal-to-noise on the wires of $\mathrm{S} / \mathrm{N} \approx 10$, with $\mathrm{LY} \approx 1 \mathrm{pe} / \mathrm{MeV}[8,72]$. The Short-Baseline Near Detector (SBND) [7] at Fermilab is capable of LY $>15 \mathrm{pe} / \mathrm{MeV}$ for all drift distances, with the average LY throughout the volume estimated to be $\approx 100 \mathrm{pe} / \mathrm{MeV}$. However, results presented here are less applicable to SBND given its surface location and small size relative to DUNE. Our electron MC sample more closely approximates the quieter conditions expected in an underground detector, free from cosmic ray pileup, capable of studying low-energy astrophysical $\nu_{e}$.

Several isolated electron samples in LArIAT are simulated with the amplitude of the wire noise tuned to achieve different $\mathrm{S} / \mathrm{N}$ ranging from $\approx 70$ (LArIAT Run II conditions) to as low as $\approx 7$. The simulated LY, or effectively the overall collection efficiency of the photodetectors, is scaled to replicate several representative values ranging from $2 \mathrm{pe} / \mathrm{MeV}$ to $100 \mathrm{pe} / \mathrm{MeV}$. We neglect the LArIAT-specific optical smearing and instead apply a smearing that replicates the expected resolution for a system capable of a single photoelectron resolution of $\sigma_{p e} / p e=0.1$ as has been achieved in many silicon photomultiplier (SiPM) devices.

We first look to the charge-based energy resolution of the electron ionization, $E_{Q}^{\mathrm{ion}}$. For each event, a proximity-based clustering procedure is repeated starting from the wire hit most closely aligned in drift coordinates $(X)$ with the true vertex of the simulated shower. Hits included within this cluster are categorized as electron-induced ionization. Here we use the deposited electron ionization energy as the true energy in the resolution. We also limit the fit to the peak region of each energy variance histogram to minimize contributions from poorly-reconstructed events - for example, cases where the clustering stops short of the 


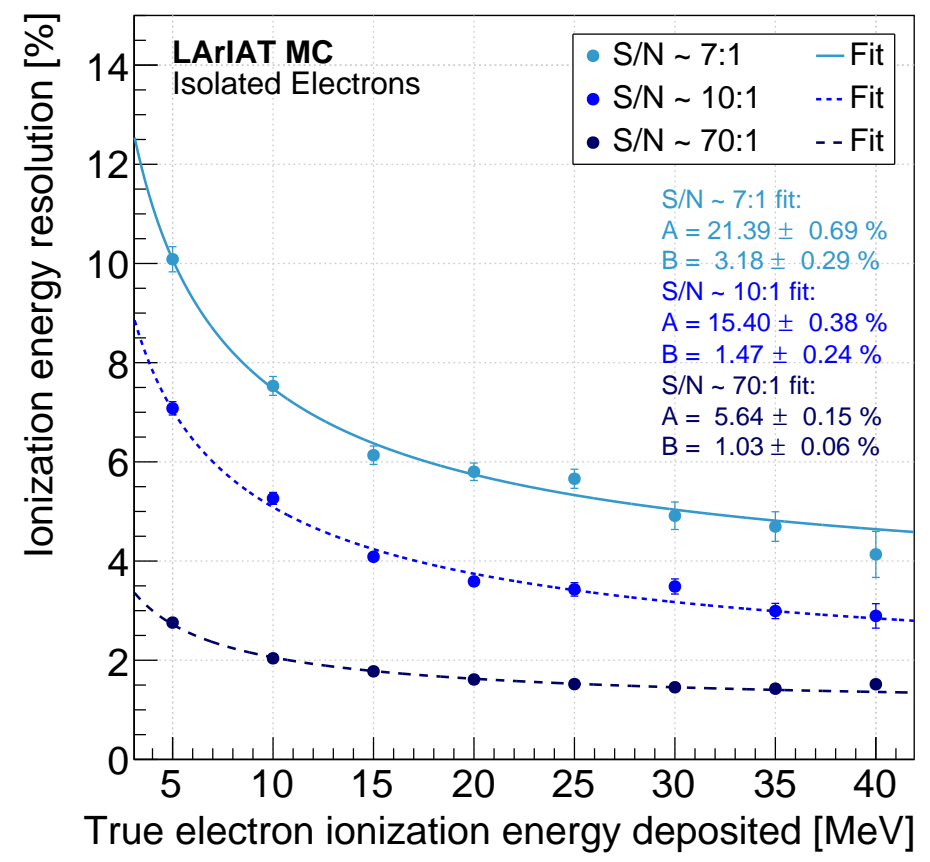

Figure 9.1: Energy resolution for the electron ionization (excluding photon deposits) for the three different simulated samples.

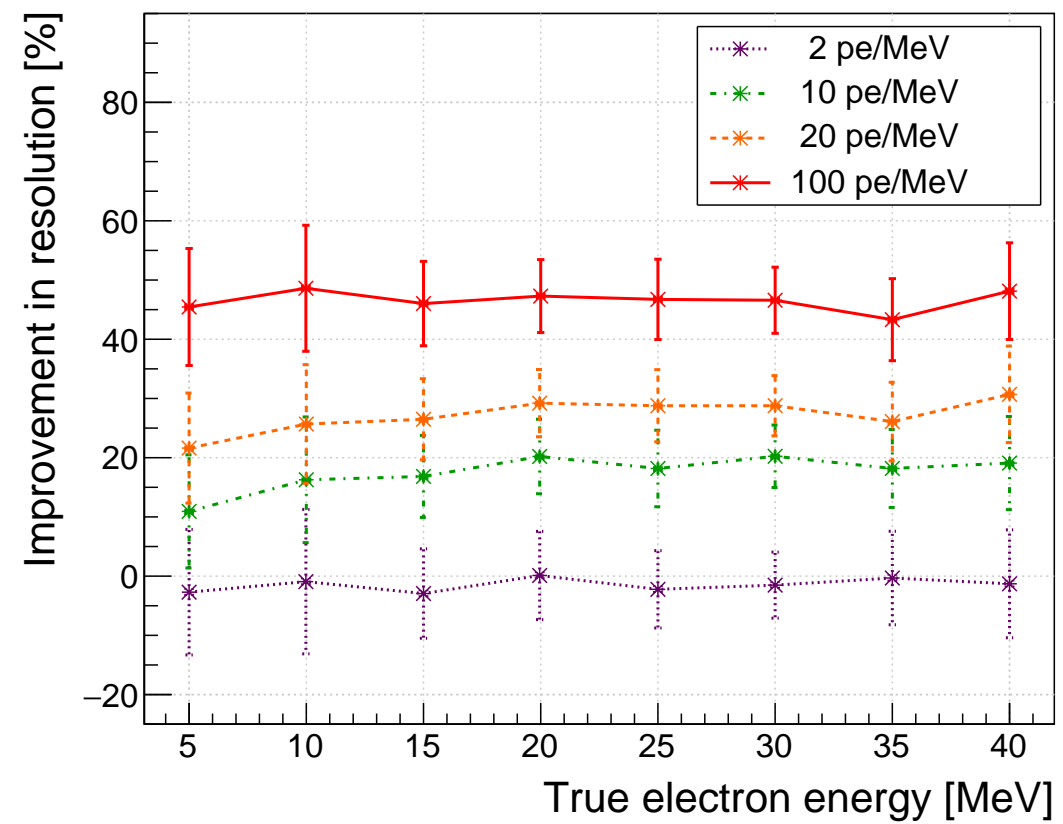

Figure 9.2: The fractional improvement in resolution with a signal-to-noise of $\mathrm{S} / \mathrm{N} \approx 10$ for the four simulated light yields. 

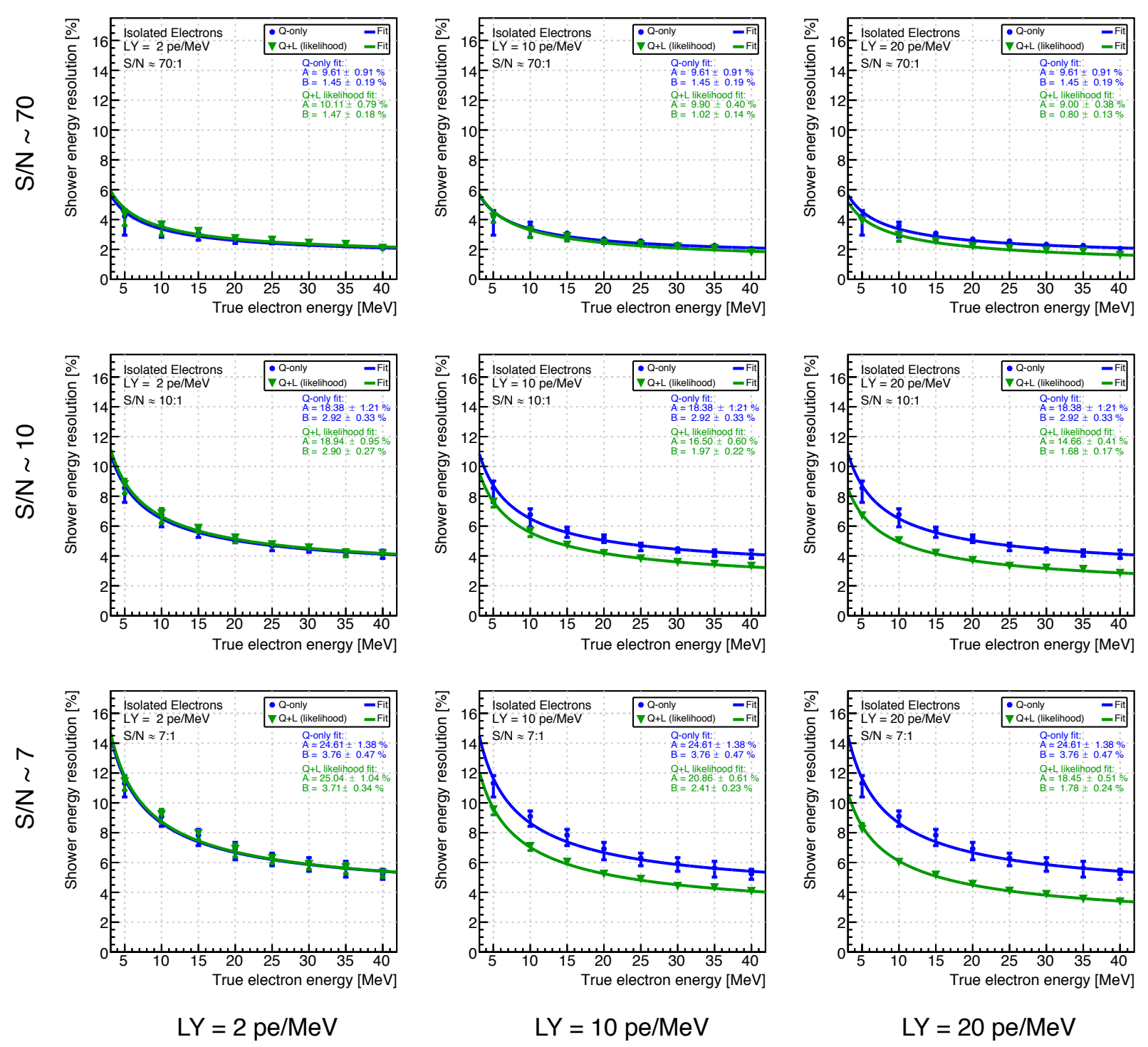

Figure 9.3: Energy resolution for charge-only energy $\left(E_{Q}\right)$ and the light-augmented likelihood energy $\left(E_{Q L}^{\text {likelihood }}\right)$ for different wire noise levels and light yields. Fits are performed to function $\sigma=A / \sqrt{E} \oplus B$. 
electron endpoint, or photon deposits are accidentally clustered together with the electron. Some fits to the energy variance distributions for $E^{\text {ion }}$ are shown in Figure B.9 (for LArIAT's nominal conditions, as an example). In Figure 9.1 the $E_{Q}^{\text {ion }}$ resolution curves for the different $\mathrm{S} / \mathrm{N}$ are plotted. The resolution behaves as expected with respect to wire noise, with the first term in the fit, which scales $\sim 1 / \sqrt{E}$, increasing considerably with noise. The flat contribution term also increases slightly - a consequence of the increased hit thresholding required at higher noise levels.

At LArIAT's operating condition, characterized by low wire noise $(\mathrm{S} / \mathrm{N}=70)$, a chargebased energy resolution of $\sigma / E=5.6 \% \oplus 1.0 \%$ is achieved, according to our simulation. At a noise level closer to what DUNE will experience, a resolution of $\sigma / E=15.4 \% \oplus 1.5 \%$ is achieved for $E^{\text {ion }}$. Since ICARUS also looked only at $E^{\text {ion }}$ in their Michel electron studies, we may compare these results to the resolution they achieved Figure 2.9. $\sigma / E=11 \% \oplus 2.5 \%$.

We now turn to the energy of the complete EM shower. PDFs for $Q$ and $L$ are again parameterized as functions of true electron energy $E_{e}$. We assume here that the resolutions on $Q$ and $L$ are independent - therefore, we only parameterize $f_{L}$ for each LY and $f_{Q}$ for each S/N. Examples of fits to the energy variance at different noise levels and light yields are shown in Figures B.10, B.11, and B.12 in Appendix B. In Figure 9.2 the relative improvement in resolution of the light-augmented likelihood energy $\left(E_{Q L}^{\text {likelihood }}\right)$ compared to the chargebased energy $\left(E_{Q}\right)$ is plotted as a function of true electron energy for the range of light yields simulated, assuming a fixed $\mathrm{S} / \mathrm{N} \approx 10$. Figure 9.3 presents the array of energy resolutions for each simulated scenario for both $E_{Q}$ and $E_{Q L}^{\text {likelihood }}$. At a light yield of $2 \mathrm{pe} / \mathrm{MeV}, L$ has virtually no impact on the energy resolution, while at light yields of $10 \mathrm{pe} / \mathrm{MeV}$ and above we find that the addition of $L$ begins to noticeably improve the energy resolution. For example, at $\mathrm{S} / \mathrm{N} \approx 10$ we see a $+10-20 \%$ improvement at $10 \mathrm{pe} / \mathrm{MeV}$, increasing to $+20-30 \%$ at $20 \mathrm{pe} / \mathrm{MeV}$ and as high as $+40-50 \%$ at $100 \mathrm{pe} / \mathrm{MeV}$. The improvement relative to the 
charge-only method is more pronounced for worse signal-to-noise [87]

It is now instructive for us to briefly relate these results to the topic of astrophysical neutrino studies enabled in DUNE. As discussed in Chapter 2 , energy resolutions of $<10 \%$ and $<7 \%$ down to $5 \mathrm{MeV}$ are claimed to be necessary in order to maximize the physics impact of $\mathrm{SN}$ and solar neutrino studies, respectively [11, 12]. At $\mathrm{S} / \mathrm{N}=10$, as seen in the middle row of Figure 9.3, the $\mathrm{SN} \nu$ requirement is achieved using only charge. However, at $\mathrm{S} / \mathrm{N}=7$, the resolution is worse than $10 \%$ for $E_{e} \lesssim 8 \mathrm{MeV}$, and a light yield of at least $10 \mathrm{pe} / \mathrm{MeV}$ is needed to meet the threshold across all energies. For solar $\nu$, the requirement is not met at the nominal $\mathrm{S} / \mathrm{N}=10$ for charge-only reconstruction and the the addition of light is necessary to some extent. The details regarding the specific ramifications of improved energy resolution each of these studies are beyond the scope of this thesis.

1. Content from this chapter is included in a paper on this analysis which is currently under internal review, with an anticipated publication date in the Spring of 2018 [87. 


\section{Conclusion}

LArIAT has demonstrated light-augmented calorimetry for low-energy electrons in a LArTPC using a sample of Michel electrons from cosmic muons. A dedicated light-based trigger was implemented to obtain this sample, and an automated reconstruction determined the charge deposited and light produced by these electron-induced EM showers.

Given complications in the Michel electron charge topology like muon-electron charge overlap, the addition of scintillation light greatly improves our ability to measure the visible energy of these events. This is reflected in the energy spectra as well as in both the RMS and the peak-fitted energy resolutions. This result implies that similarly-complicated events in LArTPCs, where the charge-energy $\left(E_{Q}\right)$ would suffer from clustering or hit-finding inefficiencies, would benefit from the collection of scintillation light. Through light, information is recovered that is otherwise lost.

We achieve calorimetric improvements even with a relatively simple likelihood fitter modeling only $Q$ and $L$ for events averaged over the whole volume. A more detailed model that incorporates additional parameters (like shower location and direction) would presumably perform even better. In principle, if a simulation is sufficiently detailed in its physics - incorporating the non-radiative quenching processes that manifest at high ionization densities (see Section 3.2 - likelihood models can be constructed that accommodate a wide variety of particle types beyond the low-energy electrons we focus on in this analysis.

Our simulation of the LArIAT detector, having been validated by data comparisons, is used to estimate the calorimetric performance of DUNE in reconstructing low-energy electrons. We find that at DUNE's baseline expectation of $\mathrm{LY} \approx 1 \mathrm{pe} / \mathrm{MeV}$, the collected light has negligible (if any) impact on energy resolution. However, at LY $=10 \mathrm{pe} / \mathrm{MeV}$, $\approx 15 \%$ improvement in resolution can be achieved for these events, with $\approx 25 \%$ improvement possible at $20 \mathrm{pe} / \mathrm{MeV}$. 
It should be mentioned that our projections of detector performance are built on assumptions of a relatively uniform LY, which LArIAT achieves through use of TPB-coated foils surrounding the active volume. A less uniform LY will require greater diligence in properly modeling the variability in photon visibility across the TPC. In addition, we assume a SiPM-like SPE resolution $(\simeq 0.1$ pe) and an optical reconstruction that incorporates photon-counting and selective integration techniques instead of full brute-force integration of charge.

We hope these results will inspire further discussion on the role light can play in LArTPCs, not just for astrophysical neutrino physics but for accelerator oscillation physics as well. A more holistic treatment of reconstruction and calorimetry that exploits the maximum amount of information possible from neutrino interactions has the potential to extend the physics reach of these beautiful detectors and impact analyses for decades to come 17

1. Content from this chapter is included in a paper on this analysis which is currently under internal review, with an anticipated publication date in the Spring of 2018 [87. 


\section{Appendix A \\ Determining the Quenched Scintillation Lifetime in Ar}

Since the measured lifetime from optical waveforms is a convolution of the lifetimes of the two LAr excimer populations as well as the four components of TPB fluorescence with lifetimes ranging from $\approx 1$ ns to as high as $3.55 \mu \mathrm{s}$ [67], a conversion is needed between measured and "true" $\tau_{t}^{\prime}$. A Monte Carlo (MC) study was performed in which PMT waveforms were generated (incorporating LAr physics, and TPB reemission, and propagation time within LArIAT) with different levels of quenching applied. A linear relationship was found relating $\tau_{t}^{\prime}$ to the measured lifetime $\left(\tau_{\text {meas }}\right)$ from fitting averaged waveforms generated (using

different $\left.\tau_{t}^{\prime}\right)$ to an exponential in the range of $0.4-2 \mu \mathrm{s}$. The linear fit to results from the $\mathrm{MC}$ study is shown in Figure A.1, with the parameterized relationship given as:

$$
\begin{aligned}
\tau_{t}^{\prime}[\mathrm{ns}] & =a \tau_{\text {meas }}+b, \\
a & =0.965 \pm 0.006 \\
b & =-41.7 \pm 8.6 \mathrm{~ns}
\end{aligned}
$$




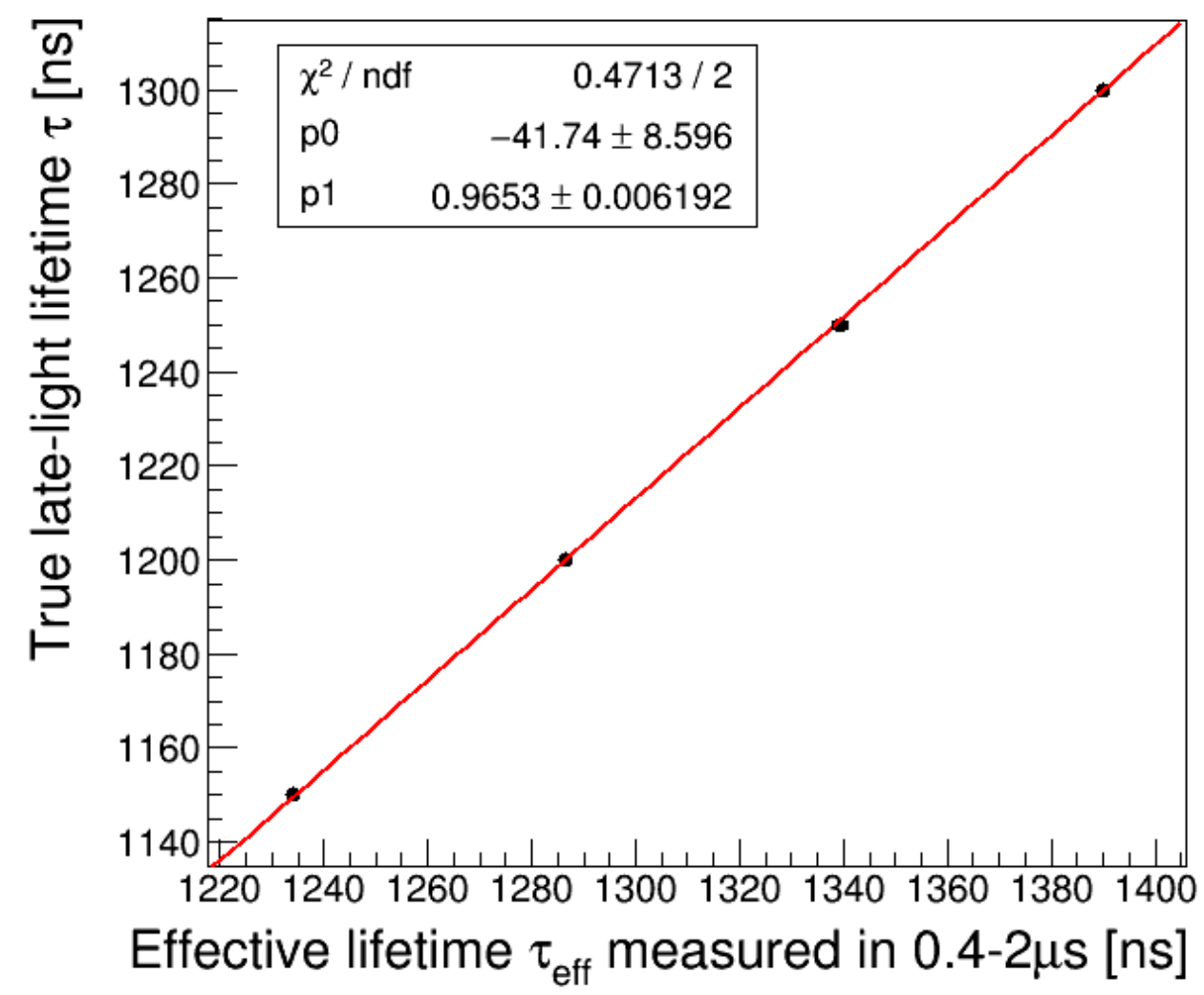

Figure A.1: Fitted linear relationship between the true quenched triplet lifetime and the measured lifetime found from fitting a single exponential to the resulting optical waveform from $0.4-2 \mu \mathrm{s}$. 


\section{Appendix B \\ Electron Energy Resolution: Supporting Plots}

The following pages include supplementary plots relevant to Chapter 8 in the discussion of energy resolution.

Figures B.1, B.2, and B.3 show the fits to the energy variance histograms for different bins of deposited energy for the Michel electron events. As discussed in Section 8.6, each distribution is fit to a Gaussian centered at the peak with a variable fit range defined by where the distribution drops below one-third the peak amplitude.

Figures B.4 and B.5 show the MC distributions of $Q$ and $L$ for isolated electrons in LArIAT under nominal Run II conditions. Each is fit to single Gaussian to determine parameterizations of the charge and light PDFs. Figures B.6, B.7, and B.8 show the energy

resolution fits for isolated electrons, binned by electron energy, for $E_{Q}, E_{Q L}$, and $E_{Q L}^{\text {likelihood, }}$ respectively.

Figures B.10, B.11, and B.12 show examples of fits to the electron energy resolution for several representative cases of wire noise levels and light yields from Chapter 9 . 

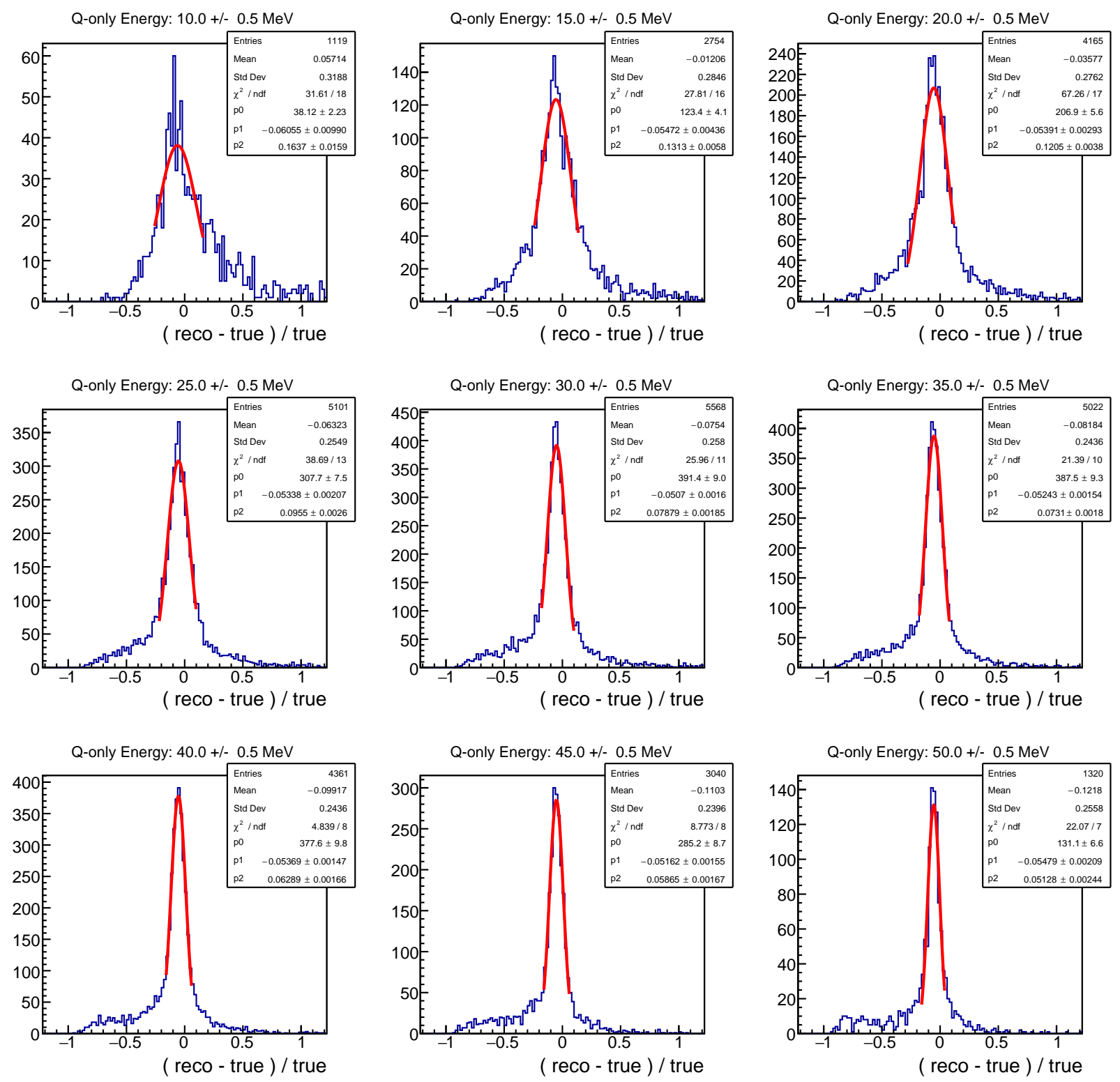

Figure B.1: Fits to the energy resolution for charge-based energy $\left(E_{Q}\right)$ of Michel electron events in LArIAT. 

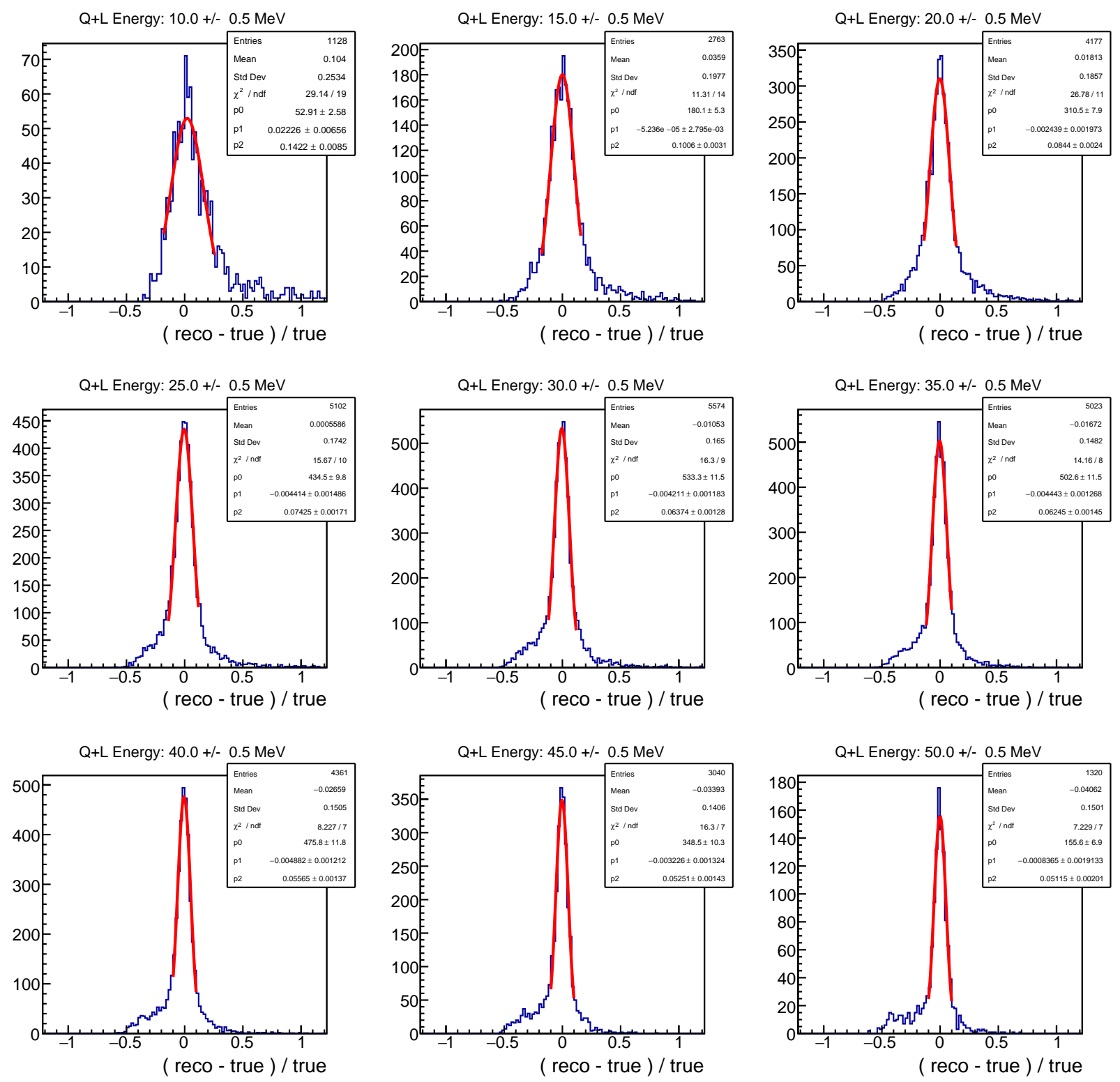

Figure B.2: Fits to the energy resolution for combined charge and light energy $\left(E_{Q L}\right)$ of Michel electron events in LArIAT. 

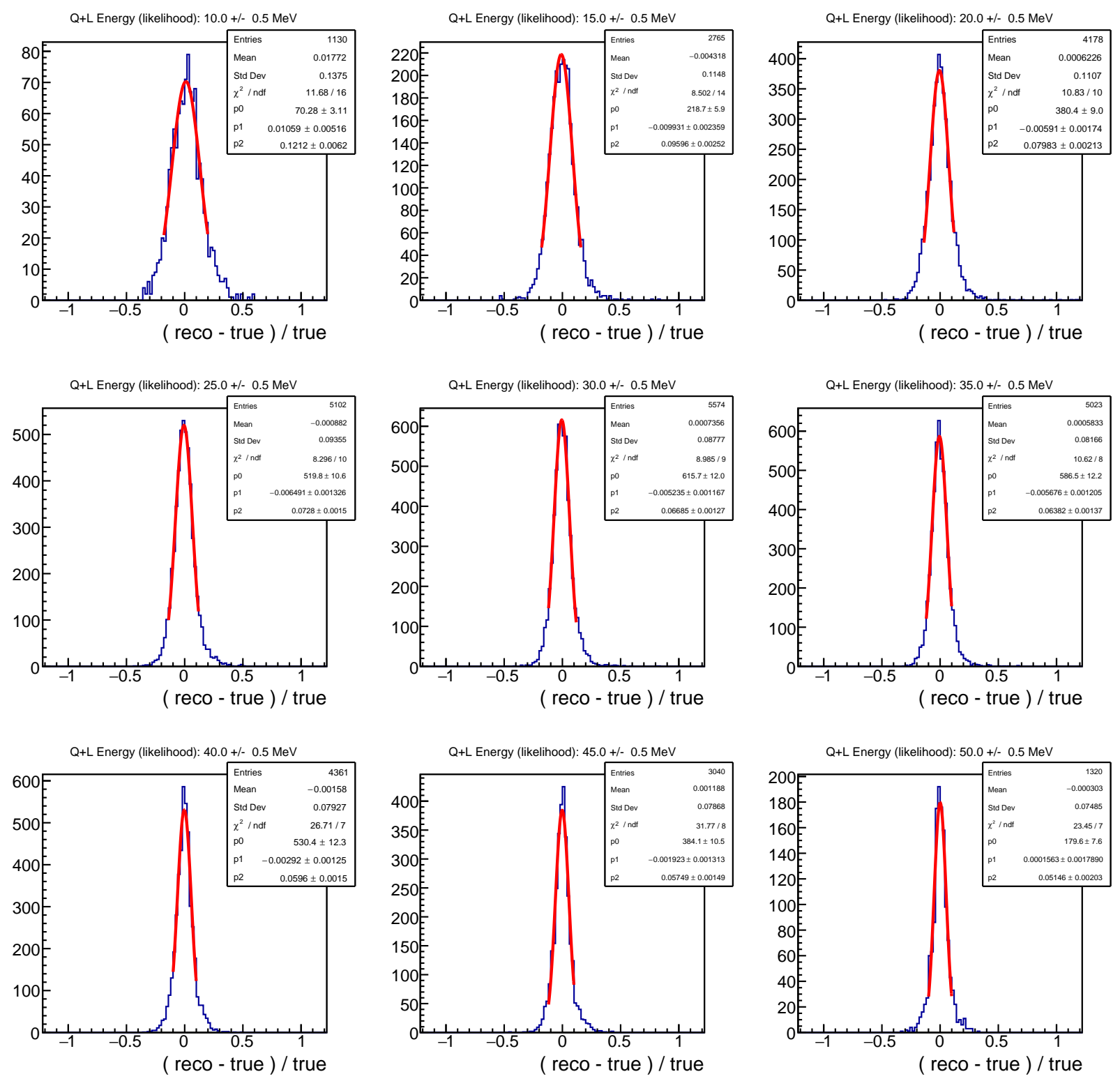

Figure B.3: Fits to the energy resolution using the maximum likelihood method ( $E_{Q L}^{\text {likelihood }}$ ) for Michel electron events in LArIAT. 

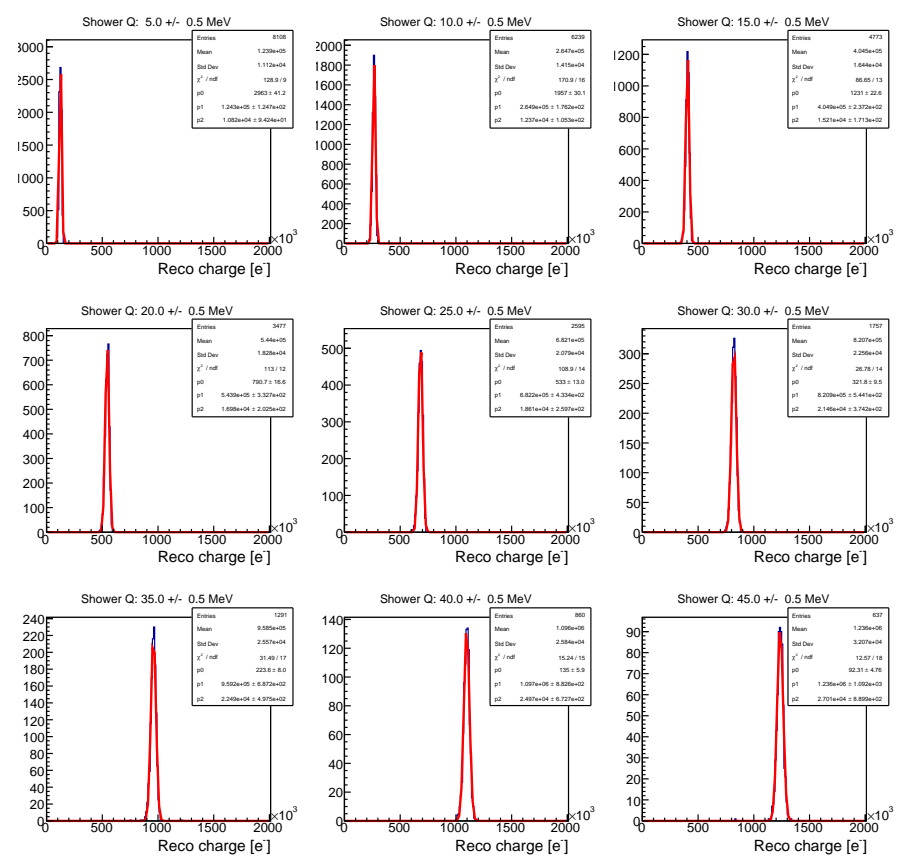

Figure B.4: Gaussian fits to $Q$ distributions for isolated electron showers for nominal LArIAT conditions at different energies.
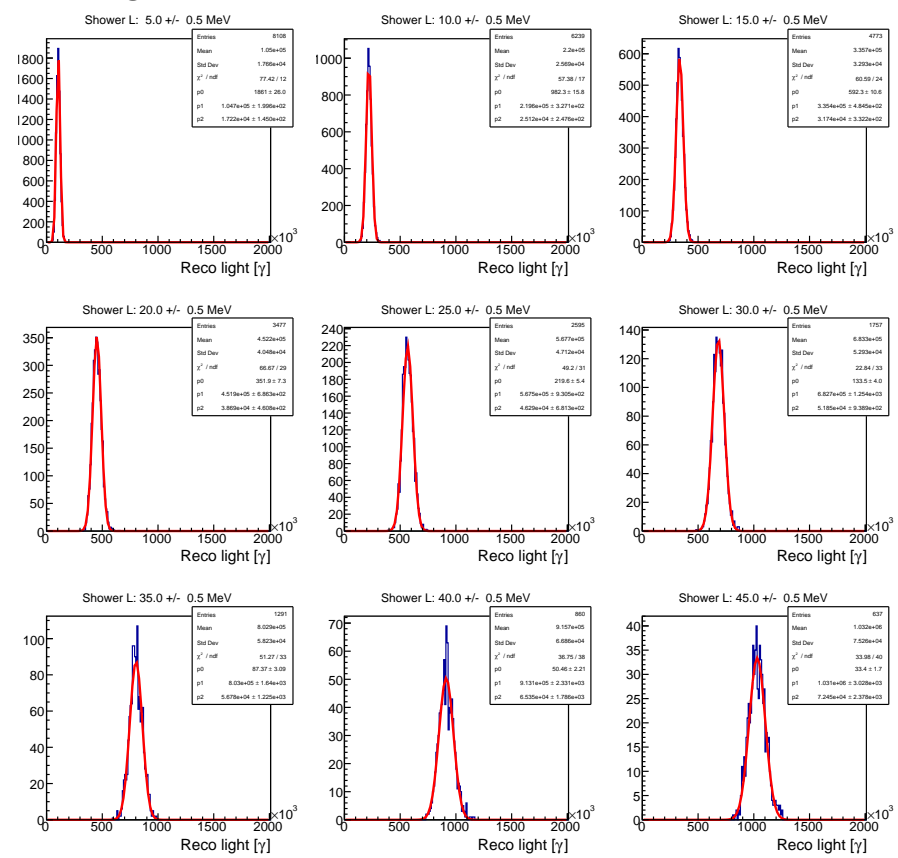

Figure B.5: Gaussian fits to $L$ distributions for isolated electron showers for nominal LArIAT conditions at different energies. 

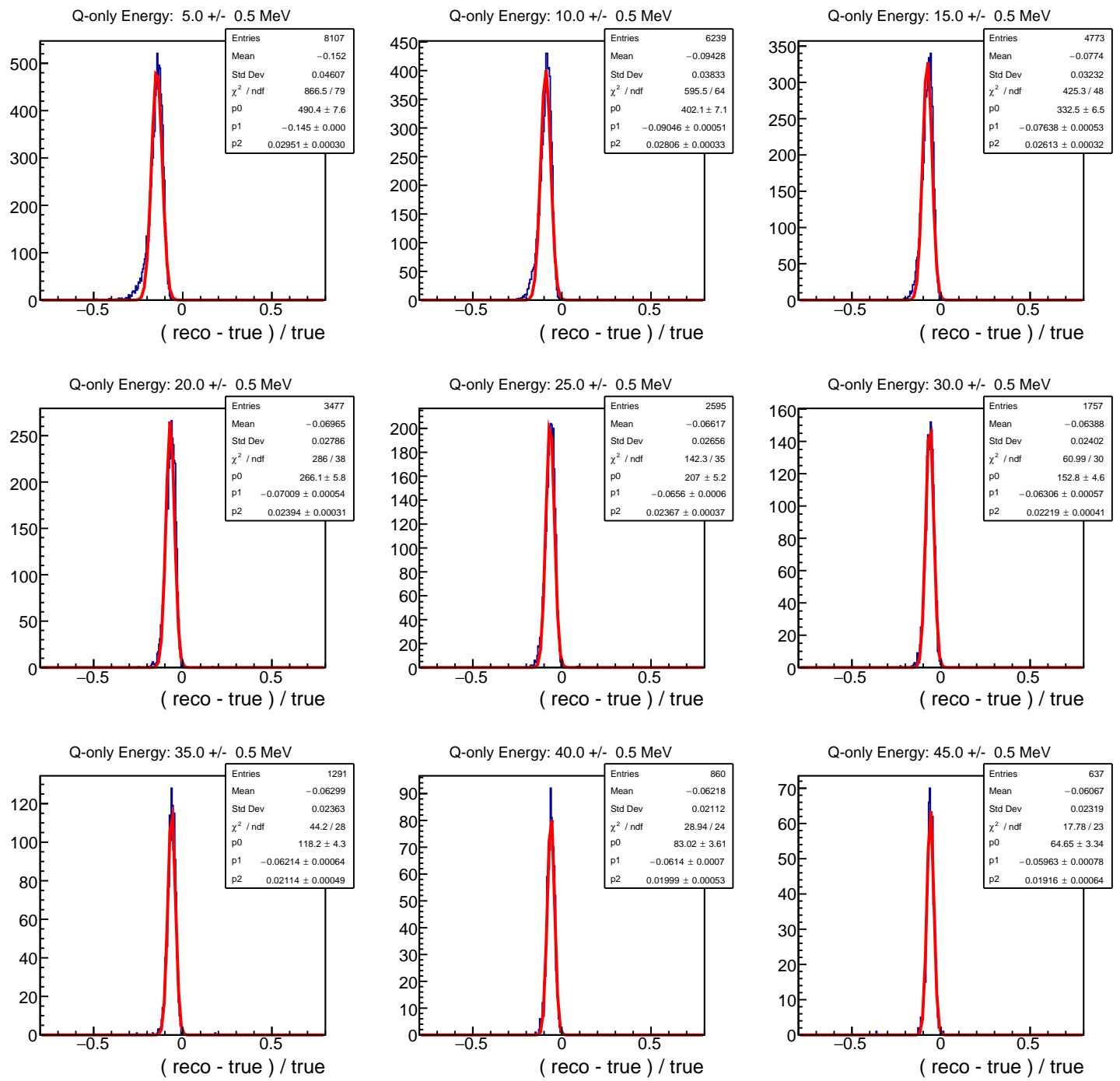

Figure B.6: Gaussian fits to the charge-only $E_{Q}$ energy variance $(\delta E)$ for isolated electron showers for nominal LArIAT conditions at different energies. 

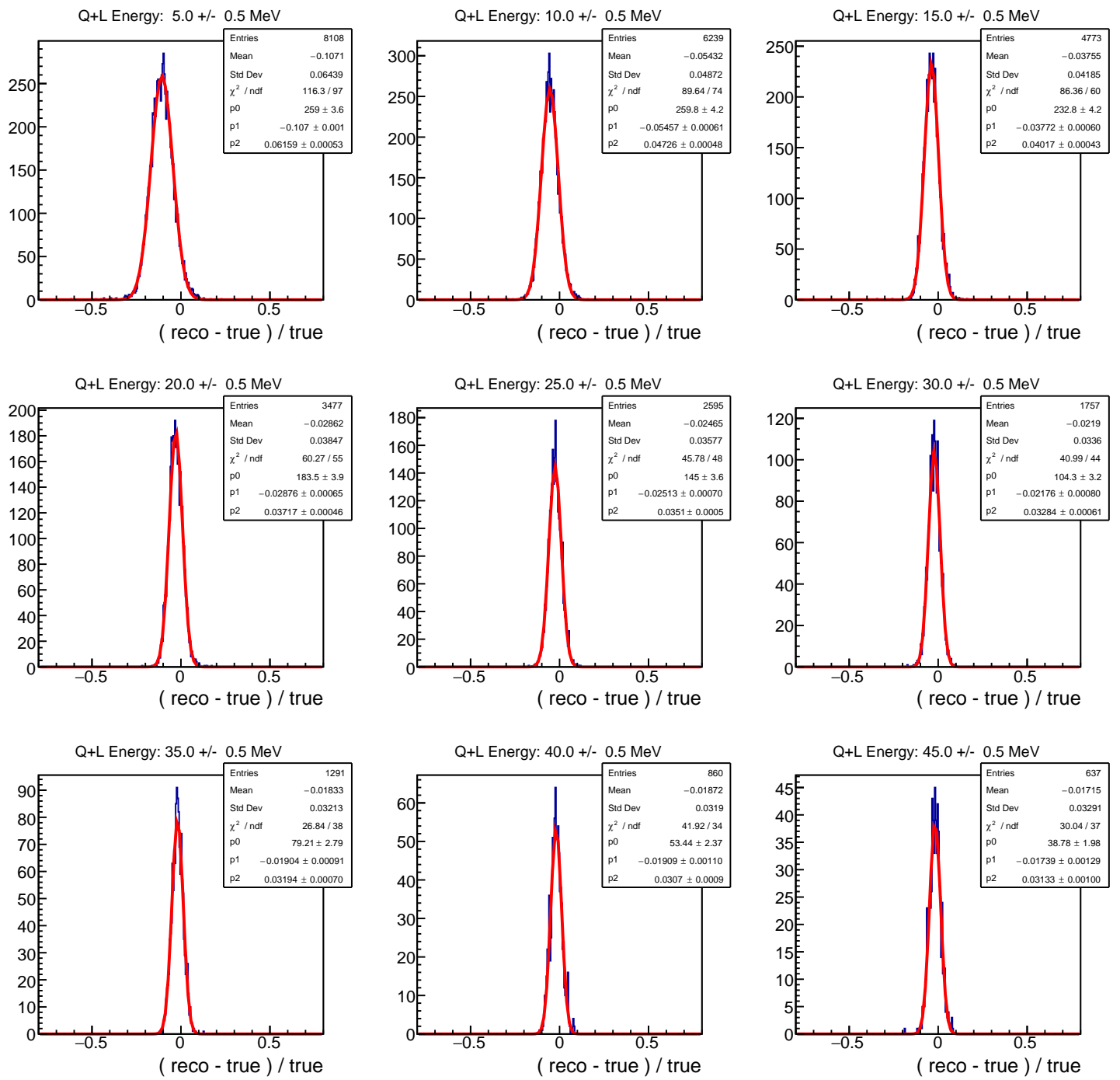

Figure B.7: Gaussian fits to $E_{Q L}$ energy variance $(\delta E)$ for isolated electron showers for nominal LArIAT conditions at different energies. 

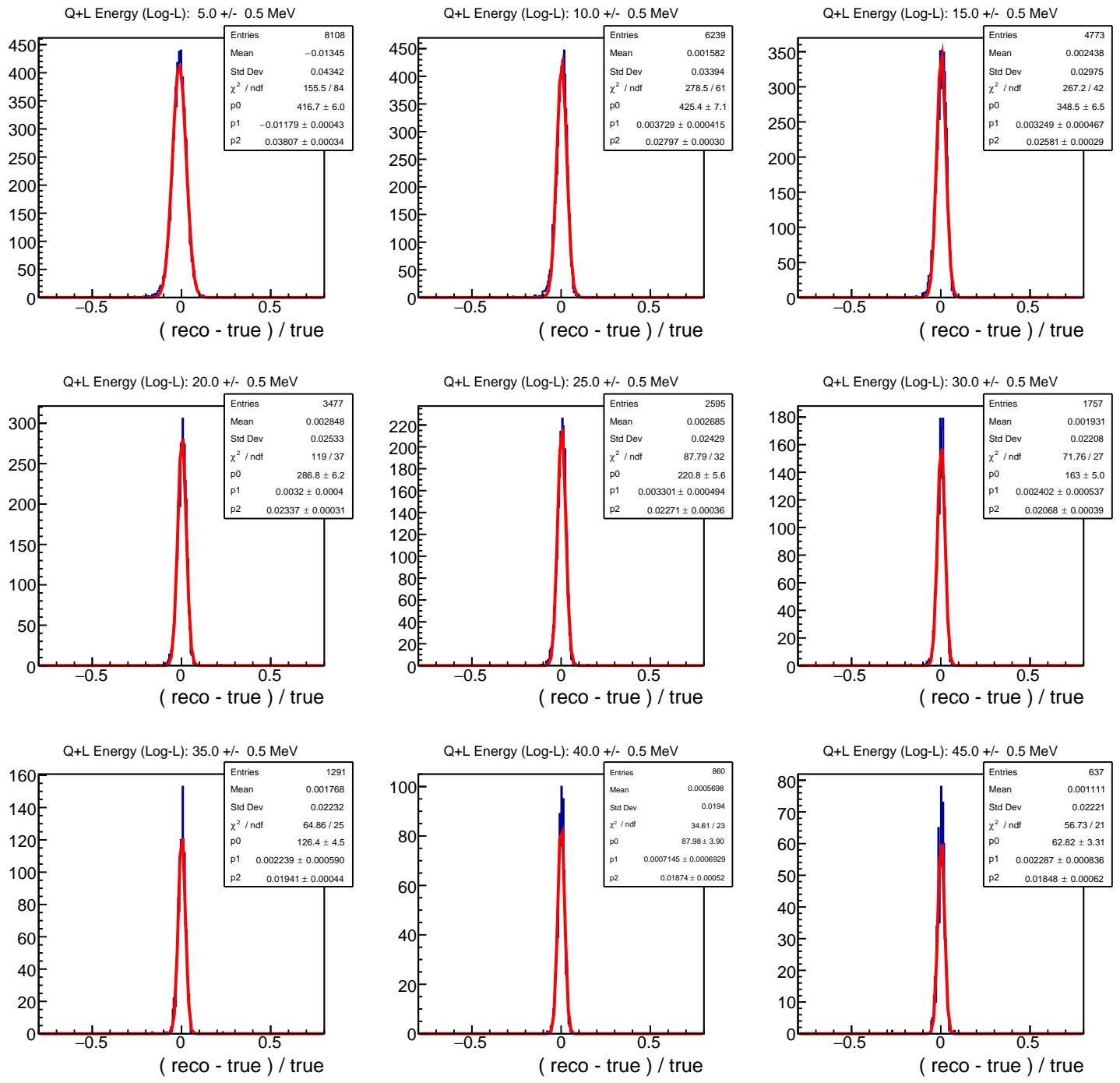

Figure B.8: Gaussian fits to the maximum-likelihood energy $E_{Q L}^{\text {likelihood }}$ energy variance $(\delta E)$ for isolated electron showers for nominal LArIAT conditions at different energies. 

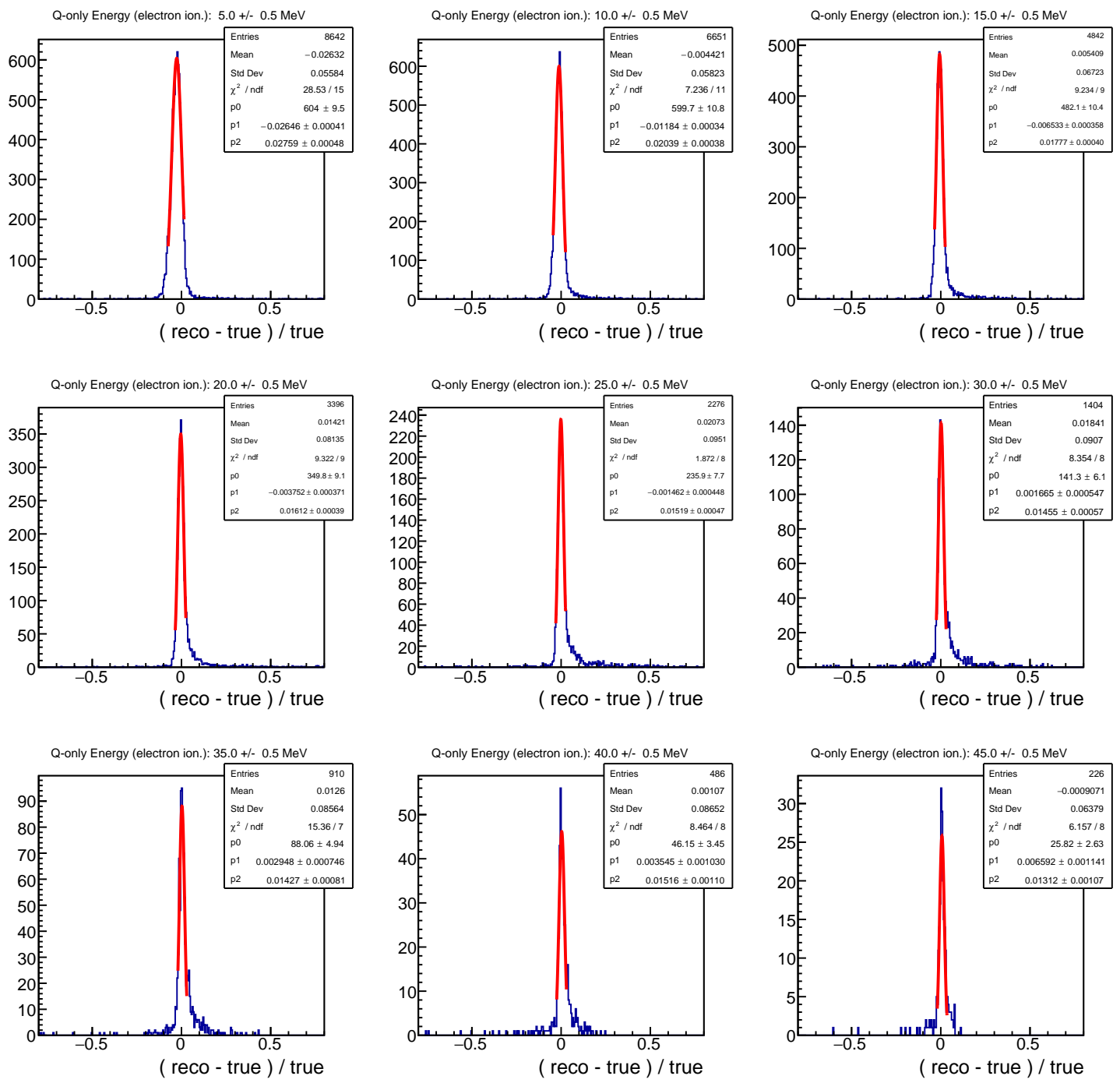

Figure B.9: Fits to energy variance $(\delta E)$ for the electron-induced ionization track $\left(E^{\text {ion }}\right)$ for nominal LArIAT conditions at different energies. 

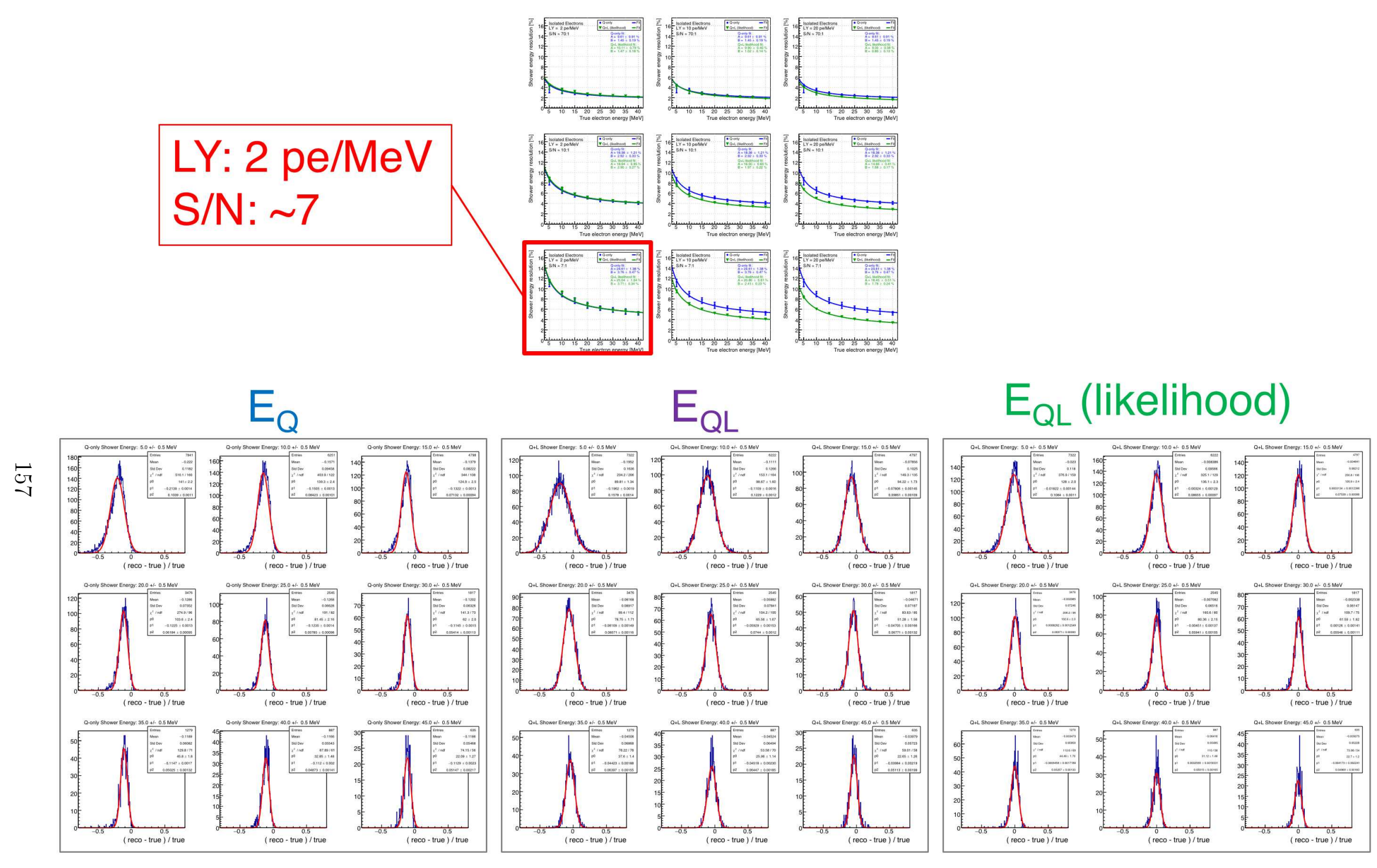

Figure B.10: Gaussian fits to the energy resolution for isolated electron showers at a light yield of 2 pe/MeV and a signal-to-noise of 7 . 


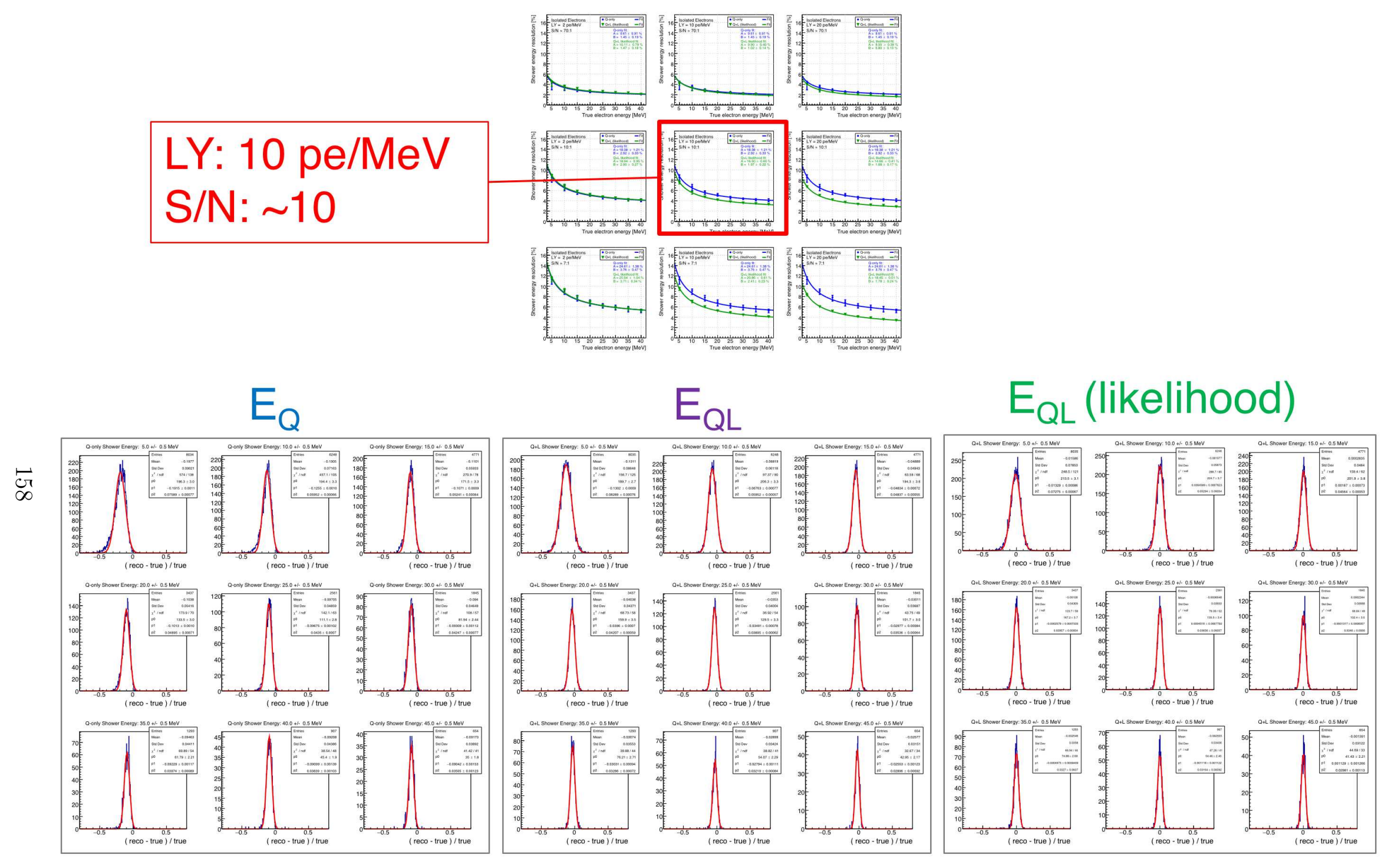

Figure B.11: Gaussian fits to the energy resolution for isolated electron showers at a light yield of $10 \mathrm{pe} / \mathrm{MeV}$ and a signal-tonoise of 10 . 

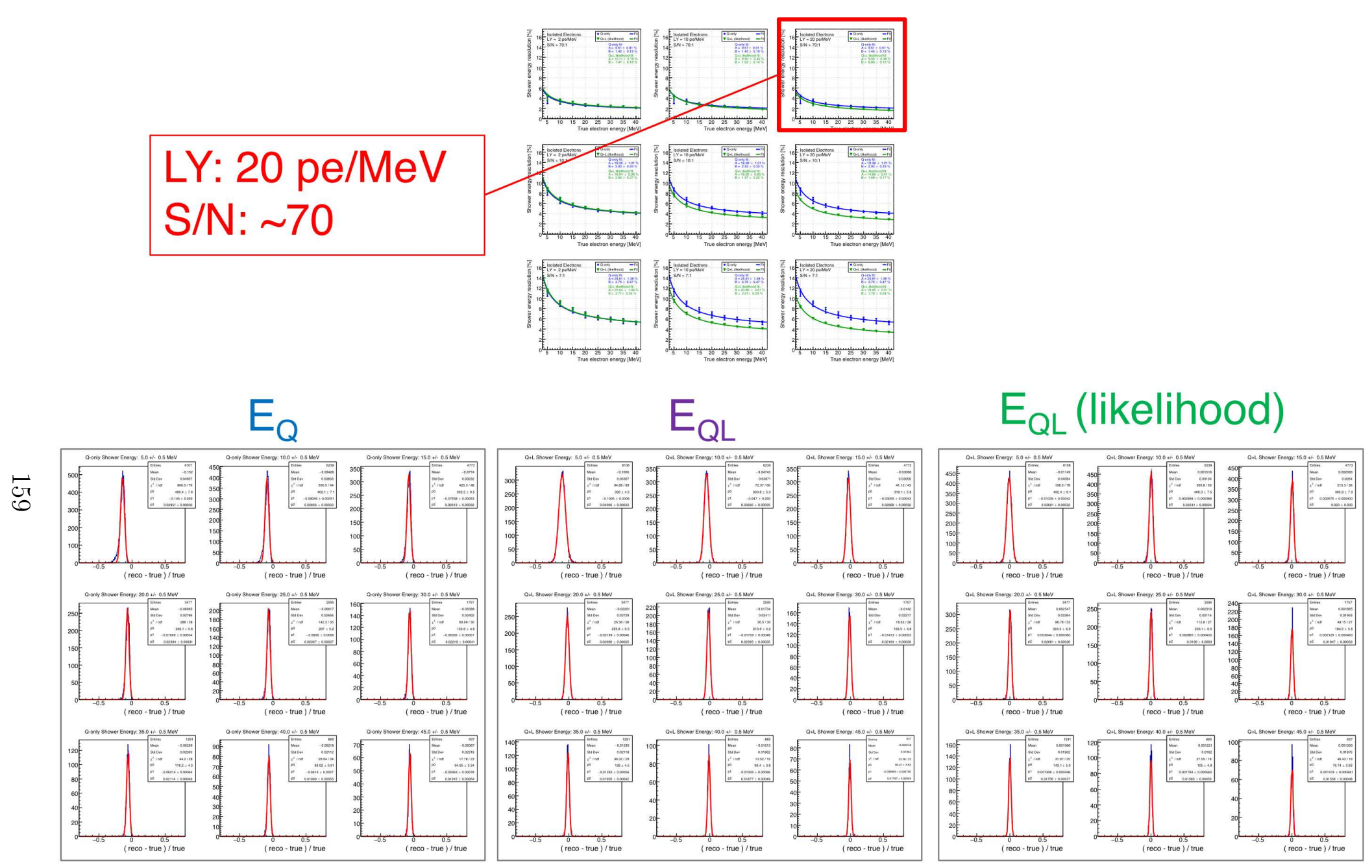

Figure B.12: Gaussian fits to the energy resolution for isolated electron showers at a light yield of $20 \mathrm{pe} / \mathrm{MeV}$ and a signal-tonoise of 70 . 


\section{References}

[1] Frank Close, Neutrino, Oxford University Press (2010).

[2] E. Fermi, An attempt of a theory of beta radiation.1, Il Nuovo Cimento, Volume 9, 1 $(1934)$.

[3] C. L. Cowan, Jr., F. Reines, F. B. Harrison, H. W. Kruse and A. D. McGuire, Detection of the Free Neutrino: A Confirmation, Science 124, 103 (1956).

[4] I. Gil-Botella, Neutrino Physics, CERN Yellow Report CERN-2013-003, pp.157-206 physics.hep-ph/1504.03551.

[5] A. Aguilar et al., Evidence for Neutrino Oscillations from the Observation of Electron Anti-neutrinos in a Muon Anti-Neutrino Beam Phys. Rev. D 6411207 (2001) physics.hep-ex:0104049.

[6] MiniBooNE Collaboration (A. Aguilar et al.), Unexplained Excess of Electron-Like Events From a 1-GeV Neutrino Beam, Phys. Rev. Lett. 102101802 (2009) physics.hepex:0812.2243

[7] R. Acciarri et al., A Proposal for a Three Detector Short-Baseline Neutrino Oscillation Program in the Fermilab Booster Neutrino Beam (2015) [physics.ins-det:1503.1520].

[8] DUNE Collaboration, Long-Baseline Neutrino Facility (LBNF) and Deep Underground Neutrino Experiment (DUNE) (2016), arXiv:1512.06148 [physics.ins-det]

[9] DUNE Collaboration (R. Acciarri et al.), Long-Baseline Neutrino Facility (LBNF) and Deep Underground Neutrino Experiment (DUNE) Conceptual Design Report Volume 2: The LBNF and DUNE Projects (2016) physics.ins-det:1601.05471.

[10] DUNE Collaboration, The DUNE Far Detector Interim Design Report Volume 1: Physics, Technology, 6 Strategies (2018) arXiv:1807.10334 [hep-ex]. 
[11] F. Capozzi, S. Weishi Li, Guanying Zhu, J. F. Beacon, DUNE as the Next-Generation Solar Neutrino Experiment, MPP-2018-214, SLAC-PUB-17199, arXiv:1808.08232 [physics.hep-ph]

[12] A. Ankowski et al., Supernova Physics at DUNE (2016), arXiv:1608.07853 [physics.hepex]

[13] F. Halzen, A. D. Martin, Quarks 8 Leptons: An Introductory Course in Modern Particle Physics, John Wiley and Sons, Inc. (1984).

[14] Wikimedia Commons, uploaded by MissMJ, PBS NOVA, Fermilab, Office of Science, United States Department of Energy, Particle Data Group, retrieved 2018-12-26, Wikipedia.org/wiki/Standard_Model.

[15] C. S. Wu et al., Experimental test of parity conservation in beta decay, Phys. Rev., 105:14131415 (1957).

[16] C. Patrignani et al. Review of Particle Physics, Chin. Phys., C40(10):100001, (2016).

[17] B. Kayser, Neutrino Physics, ECONFC040802:L004 arXiv:hep-ph/0506165 (2004).

[18] F. Capozzi et al., Neutrino masses and mixings: Status of known and unknown 3? parameters, arXiv:1601.07777 (2016).

[19] ALEPH Collaboration, Precision electroweak measurements on the $Z$ resonance, Phys. Rept. 427, 257 (2006) arXiv:0509008 [hep-ex].

[20] R. B. Patterson, Prospects for measurement of the neutrino mass hierarchy, Ann. Rev. of Nucl. and Part. Sci. 65, 177 (2015), arXiv:1506.07917 [hep-ex].

[21] W.J. Willis et al., Liquid-argon ionization chambers as total-absorption detec- tors, Nuclear Instruments and Methods, 120(2):221 236 (1974). 
[22] C. Rubbia, The Liquid Argon Time Projection Chamber: A New Concept for Neutrino Detectors, CERN-EP-INT-77-08 (1977).

[23] ICARUS Collaboration, CERN/SPSC 2002-027, LNGS-EXP 13/89 add. 2/01.

[24] R. Acciarri et al.(MicroBooNE), The MicroBooNE Technical Design Report, microboone.fnal.gov,

[25] T. Fischer, S. Whitehouse, A. Mezzacappa, F.-K. Thielemann, and M. Liebendorfer, Protoneutron star evolution and the neutrino driven wind in general relativistic neutrino radiation hydrodynamics simulations, Astron. Astrophys., Vol. 517, p. A80, 2010.

[26] M. Wurm et al., The next-generation liquid-scintillator neutrino observatory LENA, Astropart. Phys., Vol. 35, pp. 685732, 2012.

[27] R. Bionta, G. Blewitt, C. Bratton, D. Casper, A. Ciocio, et al., Observation of a Neutrino Burst in Coincidence with Supernova SN 1987 a in the Large Magellanic Cloud, Phys. Rev. Lett., Vol. 58, p. 1494, (1987).

[28] K. Hirata, et al., Observation of a Neutrino Burst from the Supernova SN 1987a, Phys. Rev. Lett., Vol. 58, pp. 14901493 (1987).

[29] I. Gil Botella and A. Rubbia, Oscillation effects on supernova neutrino rates and spectra and detection of the shock breakout in a liquid argon TPC, JCAP, Vol. 0310, p. 009, 2003.

[30] SNOwGLoBES: SuperNova Observatories with GLoBES, http://www.phy.duke.edu

[31] L. Hudepohl, B. Muller, H.-T. Janka, A. Marek, and G. Raffelt, Neutrino Signal of Electron-Capture Supernovae from Core Collapse to Cooling, Phys. Rev. Lett., Vol. 104, p. 251101, 2010, arXiv:0912.0260 [astro-ph.SR]

[32] F. An et al.(JUNO), Neutrino physics with JUNO, J. Phys. G 43030401 (2016), arXiv:1507.05613 [physics.ins-det]. 
[33] S. Amoruso et al.(ICARUS), Measurement of the $\mu$ decay spectrum with the ICARUS liquid Argon TPC, Eur. Phys. J. C33:233-241 (2004), arXiv:0311040 [physics.hep-ex].

[34] R. Acciarri et al.(MicroBooNE), Michel Electron Reconstruction Using Cosmic-Ray Data from the MicroBooNE LArTPC (2017), arXiv:1704.02927 [physics.ins-det],

[35] C. Anderson et al.(ArgoNeuT), The ArgoNeuT Detector in the NuMI Low-Energy beam line at Fermilab, JINST 7 P10019 (2012).

[36] R. Acciarri et al.(ArgoneuT), First Observation of Low Energy Electron Neutrinos in a Liquid Argon Time Projection Chamber, Phys. Rev. D 95072005 (2017), arXiv:1610.04102 [physics.hep-ex].

[37] R. Acciarri et al.(ArgoNeuT), Demonstration of MeV-Scale Physics in Liquid Argon Time Projection Chambers Using ArgoNeuT, FERMILAB-PUB-18-559-ND (2018), arXiv:1810.06502 [physics.hep-ex].

[38] M. Sorel, Expected performance of an ideal liquid argon neutrino detector with enhanced sensitivity to scintillation light, 2014 JINST 9 P10002.

[39] C. Amsler et al. (PDG), Passage of particles through matter, PL B667, 1 (2008).

[40] D.E. Groom, N.V. Mokhov, and S.I. Striganov, Muon stopping-power and range tables, Atomic Data and Nuclear Data Tables, to be published (2000).

[41] M. Miyajima, T. Takahashi, S. Konno, T. Hamada, S. Kubota, H. Shibamura, and T. Doke, Average energy expended per ion pair in liquid argon, Phys. Rev. A 9, 1438 Published 1 March 1974; Erratum Phys. Rev. A 10, 1452 (1974).

[42] S. Kubota, A. Nakamoto, T. Takahashi, S. Konno, T. Hamada, M. Miyajima, A. Hitachi, E. Shibamura, and T. Doke, Evidence of the existence of exciton states in liquid argon and exciton-enhanced ionization from xenon doping, Phys. Rev. B 13, 1649 Published 15 February 1976. 
[43] Aprile, A. E. Bolotnikov, A. I. Bolozdynya, and T. Doke, Noble Gas Detectors, WileyVCH Verlag GmbH and Co. KGaA, 2006.

[44] T. Heindl et al., The scintillation of liquid argon, Europhys. Lett. 9162002 (2010), arXiv:1511.07718 [physics.ins-det].

[45] Shen, V.K., Siderius, D.W., Krekelberg, W.P., and Hatch, H.W., Eds., NIST Standard Reference Simulation Website, NIST Standard Reference Database Number 173, National Institute of Standards and Technology, Gaithersburg MD, 20899, http://doi.org/10.18434/T4M88Q, Retrieved Dec 12, 2018.

[46] T. Doke, K. Masuda and E. Shibamura, Estimation of absolute photon yields in liquid argon and xenon for relativistic (1 MeV) electrons, Nucl. Instrum. \& Methods A 291 (1990) 617.

[47] T. Doke, A. Hitachi, J. Kikuchi, K. Masuda, H. Okada, E., Shibamura, Absolute Scintillation Yields in Liquid Argon and Xenon For Various Particles, Jpn. J. Appl. Phys. Vol. 41 (2002) pp. 1538-1545.

[48] A. Hitachi et al., Effect of ionization density on the time dependence of luminescence from liquid argon and xenon, Phys. Rev. B 27, 5279 (1983).

[49] W. H. Lippincott et al., Scintillation time dependence and pulse shape discrimination in liquid argon (2008), nucl-ex/0801.1531

[50] M. Hofmann et. al, Ion-beam excitation of liquid argon, Eur. Phys. J. C (2013) 73:2618, arXiv:1511.07721 [physics.ins-det],

[51] L. Onsager, Phys. Rev. 54, 554 (1938).

[52] G. Jaffe, The columnar theory of ionization, Ann. Phys. 42 (1913) 303.

[53] M. Wojcik, M. Tachiya, Electron thermalization and electron-ion recombination in liquid argon, Chem. Phys. Lett. 379 (2003) 20. 
[54] J. B. Birks, Scintillations from Organic Crystals: Specific Fluorescence and Relative Response to Different Radiations, Proc. Phys. Soc. A64 (1951) 874.

[55] S. Amoruso et al., Study of electron recombination in liquid argon with the ICARUS $T P C$, NIM A $523(2004) 275$.

[56] T. Doke, A. Hitachi, J. Kikuchi, K. Masuda, S. Tamada, A. Mozumder, E. Shibamura, T. Takahashi, Estimation of the fraction of electrons escaping from recombination in the ionization of liquid argon with relativistic electrons and heavy ions, Chem. Phys. Lett. $115(1985) 3434$.

[57] J. Thomas, D.A. Imel, Recombination of electron-ion pairs in liquid argon and liquid xenon, Phys. Rev. A 36 (1987), 614.

[58] R. Acciarri et al, A study of electron recombination using highly ionizing particles in the ArgoNeuT Liquid Argon TPC, 2013 JINST 8 P08005.

[59] D. M. Mei, Z. B. Yin, L. C. Stonehill, A. Hime, A Model of Nuclear Recoil Scintillation Efficiency in Noble Liquids, Astropart. Phys. 30:12-17, 2008 arXiv:0712.2470 [physics.nucl-ex]

[60] J. Lindhard, Mat. Fys. Medd. K. Dan. Vidensk. Selsk. 33, 1 (1963).

[61] A. Hitachi, in Proceedings of the Fourth International Workshop, York, UK, 2002, edited by Neil J. C. Spooner and Vitaly Kudryavtsev (University of Sheffield, United Kingdom, 2003), P. 357; in 5th International Workshop on the Identification of Dark Matter, IDM2004, Edinburgh, Scotland, 2004(unpublished).

[62] Clark, I.D., Masson, A.J. and Wayne, R.P., Molecular Physics, V 23, 995 (1972).

[63] WArP Collaboration, Oxygen contamination in liquid Argon: combined effects on ionization electron charge and scintillation light, 2010 JINST 5 P05003 
[64] G. Bakale et al., Effect of an electric field on electron attachment to sulfur hexafluoride, nitrous oxide, and molecular oxygen, J. Chem. Phys. 80, (1976), 2556.

[65] R. Acciarri et al., Effects of Nitrogen contamination in liquid Argon, 2010 JINST 5 $\mathrm{P} 06003$.

[66] A. Zani, The WArP Experiment: A Double-Phase Argon Detector for Dark Matter Searches, Adv. High Energy Phys. (2014) 205107.

[67] E. Segreto, Evidence of delayed light emission of tetraphenyl-butadiene excited by liquidargon scintillation light, Phys. Rev. C 91, 035503 (2015), arXiv:1411.4524 [physics.insdet].

[68] F. Cavanna, M. Kordosky, J. Raaf, B. Rebel on behalf of the LArIAT Collaboration, LArIAT: Liquid Argon In A Testbeam, arXiv:1406.5560v3 [physics.ins-det]

[69] R. Acciarri et al.(LArIAT), The Liquid Argon In A Testbeam (LArIAT) Experiment, currently being prepared for submission to JINST in 2019.

[70] FTBF (Fermilab Test Beam Facility), http://ftbf.fnal.gov.

[71] M. Adamowski, B. Carls, E. Dvorak, A. Hahn, W. Jaskierny, C. Johnson et al., The liquid argon purity demonstrator, 2014 JINST 9 P07005.

[72] B. Abi, et al. (ProtoDUNE Collaboration), The Single-Phase ProtoDUNE Technical Design Report, arXiv:1706.07081 [ins-det]

[73] R. Acciarri et al., Demonstration and Comparison of Operation of Photomultiplier Tubes at Liquid Argon Temperature, arXiv:1108.5584 [physics.ins-det]

[74] W. Foreman, LArIAT Light Collection System Technical Details, Internal note, 2017, LArIAT DocDB:1742. 
[75] W. Foreman, Calorimetry for Low-Energy Electrons Using Charge and Light, Internal note, 2018, LArIAT DocDB:3645.

[76] G. De Geronimo et al., Front-end ASIC for a Liquid Argon TPC, IEEE Trans. Nucl. Sci. 58 (2011) 121513761385.

[77] Marcel Bardon, et. al., Measurement of the Momentum Spectrum of Positrons from Muon Decay, Phys. Rev. Lett. 14, 449 (1965).

[78] LArSoft collaboration, E. Church, LArSoft: a software package for liquid argon time proportional drift chambers, arXiv:1311.6774 [physics.ins-det].

[79] D.M. Webber et al., Measurement of the Positive Muon Lifetime and Determination of the Fermi Constant to Part-per-Million Precision, Phys. Rev. Lett. 106:041803 (2011), arXiv:1010.0991 [physics.hep-ex],

[80] T. Suzuki, D. F. Measday, Total nuclear capture rates for negative muons, Phys. Rev. C 35, 2212.

[81] D.F. Measday, The nuclear physics of muon capture, Physics Reports 354 (2001) 234409 .

[82] A. Czarnecki et al., Michel decay spectrum for a muon bound to a nucleus, arXiv:1406.3575 [physics.hep-ph]

[83] A. Klinskih, V. Egorov, M. Shirchenko, D. Zinatulina, Muon Capture in Ar. The Muon Lifetime and Yields of Cl Isotopes, Bulletin of the Russian Academy of Sciences: Physics, 2008, Vol 72, No. 6, pp. 735-736.

[84] B. Baller, Liquid Argon TPC Signal Formation, Signal Processing and Hit Reconstruction, FERMILAB-PUB-15-458-ND, arXiv:1703.04024 [physics.ins-det].

[85] B. Baller, Trajcluster user guide, Technical report (2016). https://cdcvs.fnal.gov/ redmine/documents/1026. 
[86] M. Antonello et al., Precise 3D track reconstruction algorithm for the ICARUS T600 liquid argon time projection chamber detector, Advances in High Energy Physics, 2013:116, 2013.

[87] (LArIAT), Calorimetry for low-energy electrons using charge and light in liquid argon, paper in progress to be submitted to PRD (2018).

[88] R. Acciarri et al., Determination of the electron lifetime in LArIAT, Internal note, 2016, LArIAT DocDB:1804.

[89] R. Accierri, T. Yang, Investigation of non-uniformity observed in the Collection plane wires response to charge in LArIAT Run I, Internal note, 2017, LArIAT DocDB:1994.

[90] W. Foreman, Charge Response Calibration Using dE/dx of Stopping Cosmic Muon Tracks in LArIAT for Run I and Run II, Internal note, 2018, LArIAT DocDB:3484.

[91] C. Patrignani et al.(Particle Data Group), Chin. Phys. C, 40, 100001 (2016) and 2017 update, http://pdg.lbl.gov/2017/AtomicNuclearProperties/MUE/muE_ liquid_argon.txt.

[92] S. Agostinelli et al., GEANT4 - a simulation toolkit, Nucl. Instr. Meth. A, vol. 506, no. 3, pp. 250-303, 2003, Geant4.web.cern.ch.

[93] CMS Collaboration, Measurement of the charge ratio of atmospheric muons, Phys. Lett. B 692:83-104, arXiv:1005.5332 [physics.hep-ex].

[94] T. Heindl, PhD thesis, Technische Universität München (2011), http://mediatum.ub. tum.de/node?id=1080148.

[95] E. Grace, A. Butcher, J. Monroe, J. A. Nikkel, Index of refraction, Rayleigh scattering length, and Sellmeier coefficients in solid and liquid argon and xenon, arXiv:1502.04213 [physics.ins-det]. 
[96] V. Boccone et al., Development of wavelength shifter coated reflectors for the ArDM argon dark matter detector, 2009 JINST 4P06001, arXiv:0904.0246 [physics.ins-det],

[97] Measurements at Cracow University of Technology.

[98] LBNE/DUNE, Ibnd-DocDB:6940-v2 (Wireplane shadowing parameterization from Ben Jones).

[99] Rene Brun and Fons Rademakers, ROOT - An Object Oriented Data Analysis Framework, Proceedings AIHENP'96 Workshop, Lausanne, Sep. 1996, Nucl. Inst. \& Meth. in Phys. Res. A 389 (1997) 81-86. See also http://root.cern.ch/. 